\title{
Spectroscopy and Microreactor Technology for Single Catalyst Particle Diagnostics
}

\author{
Anne-Eva Nieuwelink
}


ISBN: 978-94-6375-997-7

Cover design: J. Zondervan

Printing: Ridderprint, the Netherlands

Copyright ${ }^{\odot} 2020$ by A.E. Nieuwelink 


\title{
Spectroscopy and Microreactor Technology for Single Catalyst Particle Diagnostics
}

Spectroscopie en Microreactortechnologie voor de Analyse van Individuele

\author{
Katalysatordeeltjes
}

(met een samenvatting in het Nederlands)

\section{Proefschrift}

ter verkrijging van de graad van doctor aan de Universiteit Utrecht op gezag van de rector magnificus, prof.dr. H.R.B.M. Kummeling, ingevolge het besluit van het college voor promoties in het openbaar te verdedigen op woensdag 16 september 2020 des ochtends te 9.15 uur

door

\section{Anne-Eva Nieuwelink}

geboren op 20 juli 1991 te Assen 


\section{Promotoren:}

Prof. dr. ir. B.M. Weckhuysen

Prof. dr. ir. A. van den Berg

Copromotoren:

Dr. ir. M. Odijk

Dr. F. Meirer

The degree is awarded as part of a Joint Doctorate with the University of Twente

This work was supported by the Netherlands Center for Multiscale Catalytic Energy Conversion (MCEC), an NWO gravitation program funded by the Ministry of Education, Culture and Science of the government of the Netherlands. 


\section{Table of Contents}

Chapter 1: General Introduction

Page 7

Chapter 2: Magnetophoretic Sorting of Single Fluid Catalytic Cracking Catalyst Particles

Page 23

Chapter 3: Single Particle Essays to Determine Heterogeneities within Fluid Catalytic Cracking Catalysts

Chapter 4: Development of a Droplet Microreactor for the High-Throughput Fluorescence-Based Detection of Single Fluid Catalytic Cracking Particle Activity

Chapter 5: High-Throughput Activity Screening and Sorting of Fluid Catalytic Cracking Catalyst Particles with a Droplet Microreactor Equipped with Dielectrophoresis and Fluorescence Detection

Chapter 6: Development of a Polydimethylsiloxane Microreactor for Performing Multiphase Hydrogenation Reactions at the Single Catalyst Particle Level

Chapter 7: Development of a Versatile Microreactor Setup for Fluorescent Product Screening to Assess Activity of Single Catalyst Particles

Chapter 8: Summary, Concluding Remarks and Outlook

Page 127

Page 37

Page 59

Page 77

Page 97

Page 113

Chapter 9: Samenvatting, Publications, Presentations, Dankwoord, and Curriculum Vitae

Page 137 


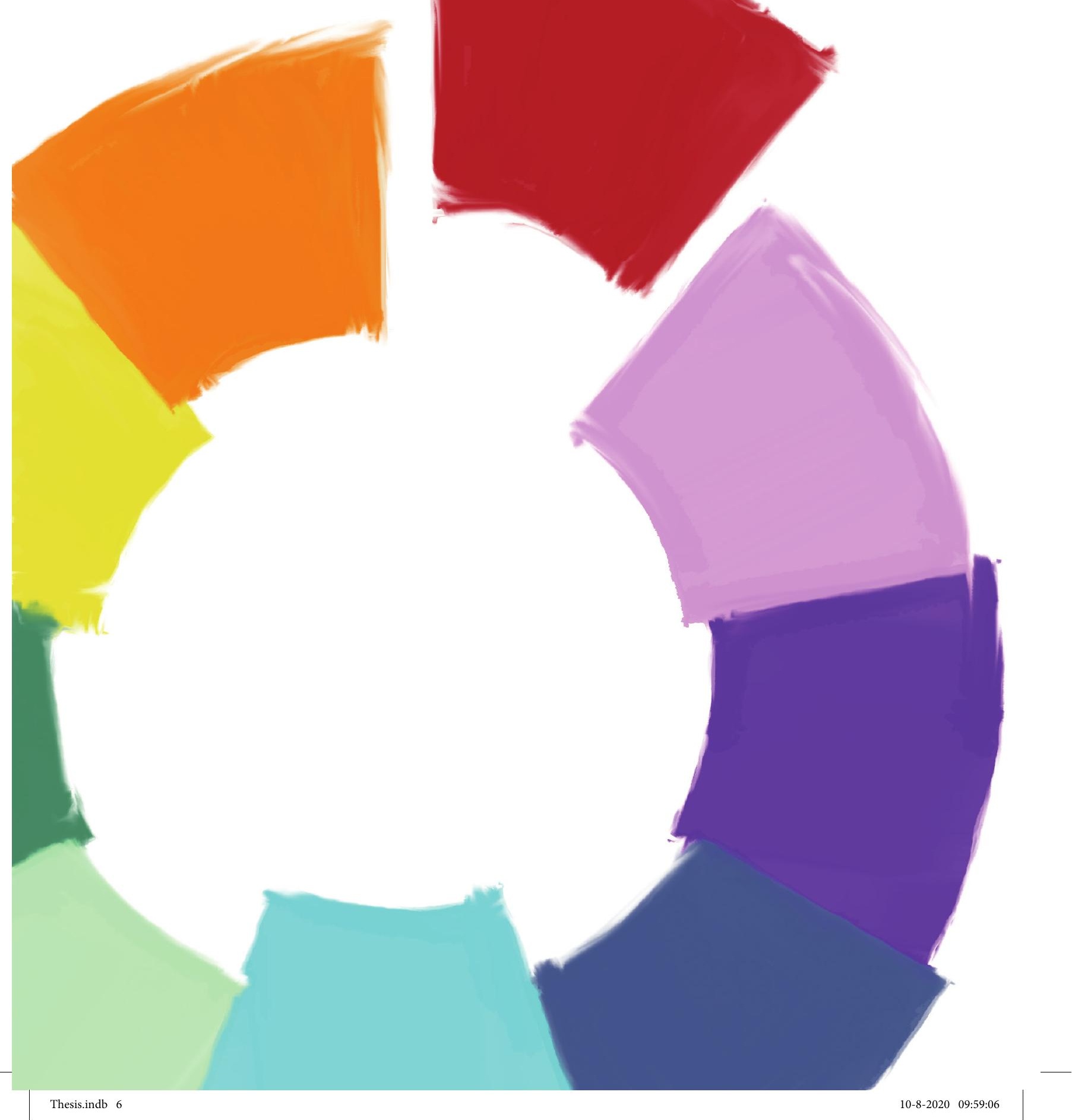




\section{Chapter 1}

General Introduction 


\subsection{Solid Catalysts and their Spatiotemporal Heterogeneities}

Solid catalysts are important materials in the chemical industry to facilitate a chemical reaction in an energy effective and selective way. They lower the activation energy of a chemical reaction without altering its thermodynamic equilibrium. That is why catalysis is involved in the production of over $80 \%$ of all chemical products. ${ }^{[1,2]}$ Solid catalysts come in many different shapes and sizes, but they have in common that they are often micrometer sized, structured particles, with a well-defined porosity. ${ }^{\ominus}$ Four important and interdependent aspects of heterogeneous catalysts are activity, stability, selectivity, and accessibility. The actual catalysis happens at the particles' active sites. This can, for example, be a spot on a metal nanoparticle or an acid/basic site. The rest of the (often porous) particle serves to provide, among other things, a large surface area and mechanical stability. The large surface area yields a good accessibility of the active sites and the mechanical stability is needed to maintain this porosity over many catalytic cycles. The activity of a catalyst is defined by its turnover frequency but becomes meaningless when a high percentage of non-desired product is produced. Vice versa, a very selective catalyst with a low activity is equally useless. Furthermore, the accessibility of active sites is of utmost importance for a high catalytic activity. However, increasing the porosity of a catalyst can decrease the particle's stability. ${ }^{[1,3]}$ To arrive at an optimal catalytic process, extensive research is needed to optimize the interplay of these four parameters. With the increasing knowledge that we have on the structure, composition and functioning of solid catalysts, we know that these complex, multi-component and hierarchical materials are generally very heterogeneous in nature. ${ }^{[4,5]}$ This inter- as well as intraparticle heterogeneity makes in-depth analysis of the catalyst material difficult by limiting the usability of bulk characterization methods on the one side, and complicating single catalyst particle analysis due to the lack of statistical relevance on the other side. ${ }^{[6]}$ We therefore need to bridge the gap between single particle and bulk catalyst characterization by developing proper techniques that can perform single catalyst particle screening in a high-throughput fashion, to provide in-depth information with statistical relevance.

In solid catalyst characterization, there are many parameters that focus on material characteristics and activity. Examples are the number of catalytic nanoparticles on a surface (the catalyst loading), their distribution, the total number of active sites on a particle, the particle's porosity, the support structure, composition and stability, as well as coke deposition during catalysis. To predict the catalyst's behavior or to study the reason for its deactivation, it is important to know all the details of the material's characteristics. A different, and complementary way to characterize a catalyst is to measure its catalytic activity towards a certain chemical product. Both characterization of the catalyst

'Throughout this thesis the wording 'catalyst particle' is used for the micrometer sized, often porous body. When referring to for example an catalytically active metal nanoparticle, the word 'nanoparticle' is used. 
parameters as well as its activity, can be done in bulk fashion or on a single particle level. For both scales, it is important to mimic the chemical environment of the catalytic reaction of choice. In the last decades, the characterization of solid catalysts via an insitu or operando approach has gained more attention. This means performing analyses while catalysts are at work. To move this field towards single particle characterization, specialized equipment is needed for in-situ measuring of the (catalytic) properties of single catalyst bodies. In other fields of research that deal with large heterogeneous samples, such as biomedical or -chemical screening of cells or proteins, screening and sorting has already been applied. For the analysis and sorting of single cells, the use of microfluidic techniques such as Fluorescence Activated Cell Sorting (FACS) or Magnetic Activated Cell Sorting (MACS) have been investigated extensively, resulting in statistically relevant single cell analyses. ${ }^{[7]}$ Cells and catalyst particles share parameters such as their size, heterogeneity and a selectivity towards product formation. Therefore, FACS or MACS can be of interest for single catalyst particle analysis and sorting. The endeavor of modifying microfluidic single cell analysis techniques to enable single catalyst particle diagnostics research is the topic of this $\mathrm{PhD}$ thesis.

\subsection{Microfluidics}

The rapidly growing research field of microfluidics has its origins in the early 1990s with Micro Total Analysis Systems ( $\mu$ TAS) for molecular biology. ${ }^{[8]}$ The field of microfluidics deals with the technology of systems that handle small amounts of fluids, using channels with sizes ranging from tens to hundreds of micrometers. Due to the use of very small sample or reagent volumes ( $\mathrm{nL}$ to $\mathrm{pL}$ ) and the possibility to perform separations of particles or droplets, as well as the added benefit of high time resolution detection, microfluidic techniques were first used for analysis. ${ }^{[9]}$

Recent advances in microfluidics open new ways to work with micro-sized structures, such as individual cells and particles. ${ }^{[10-13]}$ The small volumes and high-speed regimes of for example 2000 generated droplets per $\mathrm{s}^{[14]}$, allow experiments at the single particle level and in a high-throughput fashion, as well as better control of the parameters affecting the system, such as reaction time or mixing. ${ }^{[15-20]}$ Different fields have benefitted from microfluidics, however, for catalysis it is far from its full potential. For example, microfluidic techniques have been used for monitoring chemical reactions ${ }^{[21,22]}$ and catalyst synthesis, offering a better control of temperature and mixing of reagents, resulting in more homogeneously shaped catalysts. ${ }^{[23,24]}$ However, a great potential of microfluidics still lies in the sorting of single catalyst particles, to decrease the heterogeneity of a catalyst sample, and to select particles with special features. Microfluidics offer various passive or active techniques to sort catalyst particles based on their physical properties.

\subsubsection{Important Fluidic Parameters}

For a basic understanding of the behavior of fluids inside a microfluidic device, this 
paragraph will cover the most important parameters in microfluidics. A fluid can be described as a material that deforms continuously under shear stress. Following this definition, both liquids and gases are considered fluids. Most important fluid parameters are dynamic viscosity $(\mu, \mathrm{kg} / \mathrm{m} \cdot \mathrm{s})$, density $\left(\rho, \mathrm{kg} / \mathrm{m}^{3}\right)$ and pressure $\left(\mathrm{p}, \mathrm{kg} / \mathrm{m} \cdot \mathrm{s}^{2}\right)$. With these three terms, the type of flow in a microchannel can be described using the dimensionless Reynolds number ( $\mathrm{Re})$, which is defined as the ratio between the inertial and viscous forces:

$$
\operatorname{Re}=\rho d V / \mu
$$

The inertial force is described by the density $\left(\rho, \mathrm{kg} / \mathrm{m}^{3}\right)$, characteristic length $(\mathrm{d}, \mathrm{m})$ and the average flow velocity $(\mathrm{V}, \mathrm{m} / \mathrm{s})$. Because the characteristic length $\mathrm{d}$, is always small in microfluidic channels, Re is always small. Experimentally, it is known that $\operatorname{Re}<1$ for all microfluidic cases, which is well below the limit for operating in the laminar flow regime. Laminar flow leads to a parabolic flow profile for single phase flows inside microchannels (see Figure 1.1). Due to friction, the flow velocity at the wall is considered to be zero (noslip boundary condition).

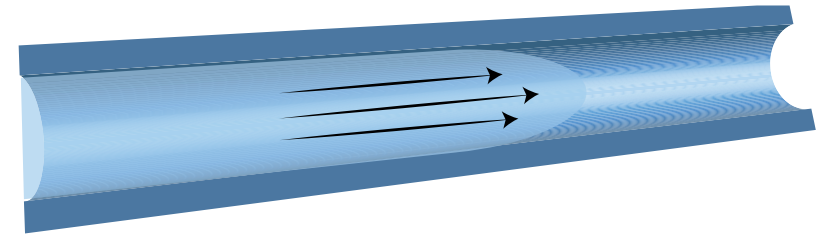

Figure 1.1: Parabolic flow profile in a microfluidic channel due to the low Reynolds number and consequently laminar flow.

Most microfluidic fabrication processes lead to rectangular channels, complicating the equation for the characteristic length $\mathrm{d}$. However, the basic flow principle for laminarity and a parabolic flow profile holds well enough in these situations. ${ }^{[25]} \mathrm{A}$ disadvantage of laminar flow is the lack of orthogonal mixing and reduced heat transfer within the channel. Furthermore, the parabolic flow profile induces diffusion of reactants or products along the length of the channel due to Taylor dispersion. ${ }^{[26]}$ Better mixing can be induced by creating special features for mixing, such as a herringbone structure ${ }^{[27]}$, or enhancing the internal recirculation by creating droplets. ${ }^{[28]}$

\subsubsection{Droplet Microfluidics}

Apart from the improved mixing, another advantage of droplet-based microfluidics over the use of continuous flows is that these droplets can serve as transport or reaction 'vessels'. This concept is very interesting for single particle diagnostics as described in more detail later. Droplets can be created actively or passively by adding two immiscible fluids to a microchannel. Passive methods make use of hydrodynamics via special geometries such as a T-junction, co-flow or flow-focusing. Active methods, not used in this work, are based on electronic, thermal, magnetic or mechanical control systems. ${ }^{[29]}$ 
a)

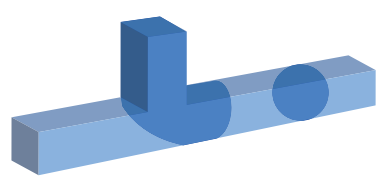

b)

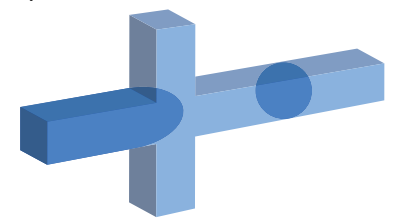

c)

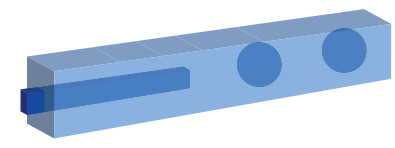

Figure 1.2: Three channel geometries for the generation of droplets are (a) a T-junction where the nonwetting dispersed phase comes in at a $90^{\circ}$ angle, (b) flow-focusing where the continuous phase is flanking the non-wetting dispersed phase, leading to a break-off, and (c) co-flow where the continuous phase is surrounding the dispersed phase.

Key parameters for the passive way of forming droplets are the microchannel geometry, the flow rate ratio and, most importantly, the capillary number $(\mathrm{Ca})$ which is defined as the ratio between the viscous stress and the interfacial tension $\left(\gamma, \mathrm{kg} / \mathrm{s}^{2}\right)$ at the liquidliquid interface:

$\mathrm{Ca}=(\mu \mathrm{V}) / \gamma$

Viscous stress is described by the dynamic viscosity $(\mu, \mathrm{kg} / \mathrm{m} \cdot \mathrm{s})$ and the velocity $(\mathrm{V}$, $\mathrm{m} / \mathrm{s}$ ) of the fluids. When $\mathrm{Ca}$ is small, the surface tension forces are dominant, leading to spherical droplets. For high values of $\mathrm{Ca}(>>1)$, the dominant viscous force leads to asymmetrically shaped droplets, caused by the flow. ${ }^{[14]}$ Therefore, tuning the flowrates and fluid viscosities influences the droplet shape. Five different droplet regimes have been determined, corresponding to different values for Ca. Droplets in the squeezing, dripping, jetting, tip-streaming and tip-multi-breaking regime all have different shapes and sizes. ${ }^{[30]}$ In general, the formation of droplets involves the deformation of the liquid-liquid interface, initiated by fluid instabilities. The different shapes and sizes also depend on many other factors, including the geometry of both the channel and droplet generator, the temperature and the presence of surfactants. In the squeezing regime highly monodisperse droplets with a size larger than the channel dimensions (plugs) are formed. In the dripping regime, with higher Ca values, monodisperse droplets smaller than the channel dimensions are formed. In the jetting regime, the viscous forces dominate even more, leading to polydisperse droplets. Droplet breakup happens further in the channel after a mode with parallel flows of the continuous and dispersed phase. The tip-streaming regime forms sub-micrometer droplets and the tip-multi-breaking regime forms sequences of droplets with an increasing size. The polydispersity of the flows makes these final three regimes unsuitable for particle sorting. ${ }^{[30]}$

When we use droplet microfluidics to encapsulate single catalyst particles inside single droplets, we create micro-environments (reaction 'vessels') of minimal volumes in the $\mathrm{nL}$ to $\mathrm{pL}$ range. The reaction products of the encapsulated catalyst particle are collected in the droplet, allowing the monitoring of catalytic activity for individual catalyst particles. Furthermore, the smaller the droplet, the lower the amount of solvent present to dilute the product formed. This is beneficial for measuring low concentrations of reaction products. This allows for single particle catalytic testing in a controlled way: 
the residence time of the droplet in the microreactor is related to the reaction time, and in-situ spectroscopy measurements can be used to analyze the products inside the droplets at multiple positions along the channel.

In previous research regarding single cell analysis ${ }^{[31]}$, the encapsulation of particles (or, more precise, cells) in droplets has proven to be challenging. The desire to have only one particle in each droplet led to the use of very low particle concentrations. Poisson statistics can be used to determine what concentration of particles is needed to have as often as possible only one particle (and not more) in a droplet, using random encapsulation. ${ }^{[32]}$ This statistical distribution models the probability for an event to repeatedly occur at a fixed time or space interval and, therefore, determines the success rate of having a single particle in a droplet. A side effect of the use of low concentrations of particles is that a large number of droplets will remain empty. ${ }^{[33]}$ Previous research shows that for cells it is possible to have a more deterministic instead of random success, overcome Poisson statistics by hydrodynamically aligning them in a spiral-shaped channel, due to Dean flow. ${ }^{[34]}$ The spacing between the cells and the flow rate then determines the amount of cells per droplet. ${ }^{[35]}$

\subsubsection{Microfluidic Characterization}

After encapsulating the catalyst particles of interest, it is important that the microscopic characterization technique used can perform a fast screening of all individual droplets that flow past the detector. The analysis method, therefore, needs to be fast, but more importantly compatible with the microreactor material. For example, not all microreactor materials are transparent for UV or IR radiation, complicating potential UV-vis or IR spectroscopy measurements. Also, high sensitivity is needed to allow for single catalyst particle detection. The small dimensions of microfluidic channels in combination with fast traveling droplets have shown to introduce problems regarding the reliability of the UV-vis absorbance measured. ${ }^{[36]}$ For example, the sample volume (a single droplet) is very small, limiting the total absorbance of UV-vis radiation. Furthermore, the fast movement of the droplets requires a short acquisition time, limiting the total absorbance even further.

A technique with a high sensitivity and thus high potential for the analysis of small volumes is surface enhanced Raman spectroscopy (SERS). In contrast to regular Raman spectroscopy, which has a too low sensitivity, this relatively new technique has been applied to microfluidics by dispersing SERS active colloids in the analyzed liquids, or using a nanostructured surface inside the microchannels for signal enhancement. ${ }^{[37,38]}$ For a detailed explanation of SERS and its application to catalysis, the reader is referred to recent literature. ${ }^{[39]} \mathrm{A}$ disadvantage of SERS is the need for hotspots and the possibly inhomogeneous enhancement per hotspot. Also, for single particle diagnostics, SERS is limited to measuring potential dissolved products in the droplet itself. Depositing SERS 
active colloids on the catalyst particles will lead to inhomogeneous distributions and thus inhomogeneous hotspots. This complicates comparing droplets within the sample.

The fastest and most commonly used analysis method involves fluorescence spectroscopy. Fluorescent labels can be used to create tracers for multiple parameters such as catalyst activity or accessibility and with the use of a Photo Multiplier Tube (PMT), proper filters and a high enough fluorescence quantum yield of the label, the fluorescence detection only takes nanoseconds. ${ }^{[40]}$ In biochemistry, the use of fluorescent dyes for the labelling of proteins or cells is already very common. Low concentrations of product can be detected rapidly, and by translating the fluorescence intensity to a signal for a sorting technique, a quick droplet sorting system can be put together. ${ }^{[41]}$ On-chip fluorescence detection is often combined with a second technique such as impedance spectroscopy. This second electronic technique can, for example, be useful for the analysis of particles or cells. A fluorescence signal can, in this case, be correlated to a sensitive measurement of the size, morphology or surface charge of single cells, obtained from impedance spectra. ${ }^{[42,43]}$

So far, research on microfluidic catalyst characterization has not been focused on droplet-based single particle screening. Two main lines of research can be determined: continuous flow systems using a microreactor with a heterogeneous catalysts coated on the channel walls on the one hand, and droplet-based systems with dispersed catalyst nanoparticles (NP) or homogeneous catalysts on the other hand. In a recent review by Yue ${ }^{[44]}$ many wall-coated microreactors were summarized. Most of them consisted of Au or Pd based catalysts for hydrogenation or oxidation reactions. The preparation of these coated catalysts was mainly done from inserting precursor solutions in the microchannel, and the catalytic activity was measured by online HPLC or GC monitoring. ${ }^{[4-47]}$ To the best of our knowledge, no spectroscopy or microscopy was performed on the microchannels themselves. For systems with solely fluid flows, there is some literature available about the in-flow (micro)spectroscopic analysis of reaction products. For example, the absorbance of nanoparticle formation during an on-chip droplet-based synthesis was measured over time by coiling tubing around a heating device. By measuring at different loops, the nucleation and growth of $\mathrm{PbS}$ nanoparticles could be followed. ${ }^{[48]}$ Also, more difficult characterization techniques have been implemented with droplet microfluidics. For example, in-situ X-ray diffraction (XRD) or attenuated total reflectance-infrared (ATRIR) spectroscopy using dedicated microfluidic chips with large X-ray or IR transparent windows have been reported. ${ }^{[22,49]}$

For the screening of individual catalyst particles in droplets a vital property of the characterization techniques used is the simultaneous collection of spectroscopic and optical data. The properties of the droplet can, in this way, be correlated to the presence or absence of a catalyst particle. This is important due to the high fraction of empty droplets that is likely present. 


\subsubsection{Particle Sorting}

After the analysis of droplets with particles, a next step could be sorting out the, for example, highly fluorescent ones. There are many ways to perform microfluidic droplet sorting, both passively and actively. Passive techniques do not rely on differences in spectroscopic read-outs, but make use of filtration, hydrodynamic spreading or inertial forces to sort by size, density or shape. One of these techniques, magnetophoresis, will be used later in this PhD thesis to sort Fluid Catalytic Cracking (FCC) catalyst particles based on their magnetic moment. More details can be found in Chapter 2. For active sorting, a spectroscopic trigger is needed to activate electric fields, acoustic waves, optical forces or mechanical valves. ${ }^{[10,11,50]}$ One of the electrokinetic methods is dielectrophoresis (DEP), described by Gascoyne et al. as the electric equivalent of magnetophoresis. ${ }^{[51]}$ When a non-uniform electric field is applied over a microchannel, a force along the gradient of the field lines acts on the droplets or particles in the channel due to polarization. As a result, the particle or droplet will move. ${ }^{[52]}$ This technique has been used in Chapter 5 to manipulate highly fluorescent FCC catalyst particles in droplets, sorting the droplets containing the catalyst particles that have been determined by fluorescence as having the highest activity. Figure 1.3 shows the concepts of magnetophoresis and DEP.

a)

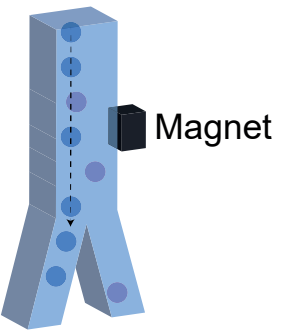

b)

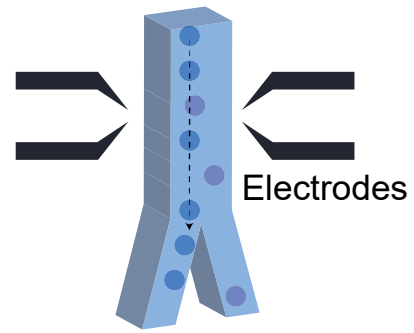

Figure 1.3: Schematic representation of (a) magnetophoretic and (b) dielectrophoretic sorting where particles that are manipulated (purple) move out of their flow stream and end up in a different outlet.

\subsection{Catalytic Systems}

In this thesis, the (microfluidic) single catalyst particle analysis of several catalytic systems is showcased. In literature, solid acids, metal oxides, and supported metals are listed as the main types of heterogeneous catalysts. ${ }^{[3,53]}$ We have tried to use a wide scope of materials, to show the applicability of microfluidic analysis for in-flow heterogeneous catalysis. However, it is difficult to cover the whole field. Most of the chapters in this thesis focus on the Fluid Catalytic Cracking catalyst, with a solid acid as active material. Also, we will use a supported Pd catalyst for a hydrogenation reaction, and finally, we describe a hydrotalcite catalyzed Knoevenagel condensation.

\subsubsection{Fluid Catalytic Cracking Catalyst}

A catalyst system that is very suitable as a showcase for single particle sorting using microfluidics, is the Fluid Catalytic Cracking (FCC) catalyst. ${ }^{[1,54,55]}$ FCC is one of the 
processes in oil refinery that uses the residue of crude oil distillation as a feedstock. It is the main chemical process to produce gasoline and propylene from Vacuum Gas Oil (VGO) and Heavy Gas Oil (HGO) with the use of a solid catalyst material. Catalytic cracking of crude oil fractions was invented by Eugene Jules Houdry and has been operating in its present form since the 1940s. ${ }^{[56]}$ The multicomponent and hierarchically structured catalyst used consists of spray dried porous spheres with an average diameter of 50-150 $\mu \mathrm{m}$ and contains next to zeolite also a clay and binder material, such as silica and alumina. The catalyst design is such that the mixture of hot catalyst and VGO/HGO feedstock behaves like a liquid; hence the name Fluid Catalytic Cracking (FCC). ${ }^{[57,58]}$ The main active sites that catalyze the cracking of VGO are the Brønsted acid sites in the zeolite material, but the large oil molecules are pre-cracked in the matrix of the FCC particle, prior to entering the crystalline micropores of the zeolite. Commonly employed zeolites in the FCC process include the synthetic ultra-stable zeolite Y (ultrastable Y or US-Y, possessing the FAU framework structure) and, to a lesser extent, zeolite ZSM-5 with the MFI framework structure. Due to the smaller micropore structure of ZSM-5 in comparison with that of US-Y (i.e., $5.6 \AA$ vs. $7.4 \AA$ ), zeolite ZSM-5 increases the FCC catalyst selectivity towards propylene. They are added to the FCC catalyst inventory as separate FCC catalyst particles, and are visually not distinguishable from normal FCC catalyst particles containing zeolite US-Y.[59,60]

During the FCC process, the cracking is performed by the hot FCC catalyst in the riser reactor, after which the formed products are separated from the catalyst using cyclones. Remaining carbonaceous species on the catalyst material, originating from side reactions during cracking, block the active sites, preventing further cracking activity. The spent catalyst is, therefore, subjected to a regeneration procedure through hightemperature calcination, making the FCC particles available for a consecutive cycle of crude oil cracking. ${ }^{[59]}$ The FCC particles can, however, also deactivate irreversibly due to the accumulation of metals originating from the crude oil feedstock, with $\mathrm{Ni}, \mathrm{Fe}$ and $\mathrm{V}$ being the most notorious ones. While $\mathrm{Ni}$ and $\mathrm{V}$ decrease the yield by promoting e.g. coke formation, Fe supposedly deactivates the particles by creating a $\sim 1 \mu$ m shell of reduced porosity on the particles' outer surface, either via vitrification of the particle matrix in the presence of Fe and/or co-deposition of silica. ${ }^{[61-70]}$ Also, the harsh steaming conditions during catalyst regeneration induce dealumination of the embedded zeolite material, thereby decreasing the amount of available Brønsted acid sites. Figure 1.4 gives a schematic representation of the cracking process and catalyst deactivation. To preserve the overall activity in a reactor, part of the spent catalyst material is constantly being replaced with fresh FCC catalyst particles, leading to an equilibrium in the reactor with a mixture of FCC catalyst particles with different ages and degrees of deactivation. This mixture is called the equilibrium catalyst, further denoted as ECAT. ${ }^{[54]}$

The previously mentioned deactivation processes, involving metal accumulation and 


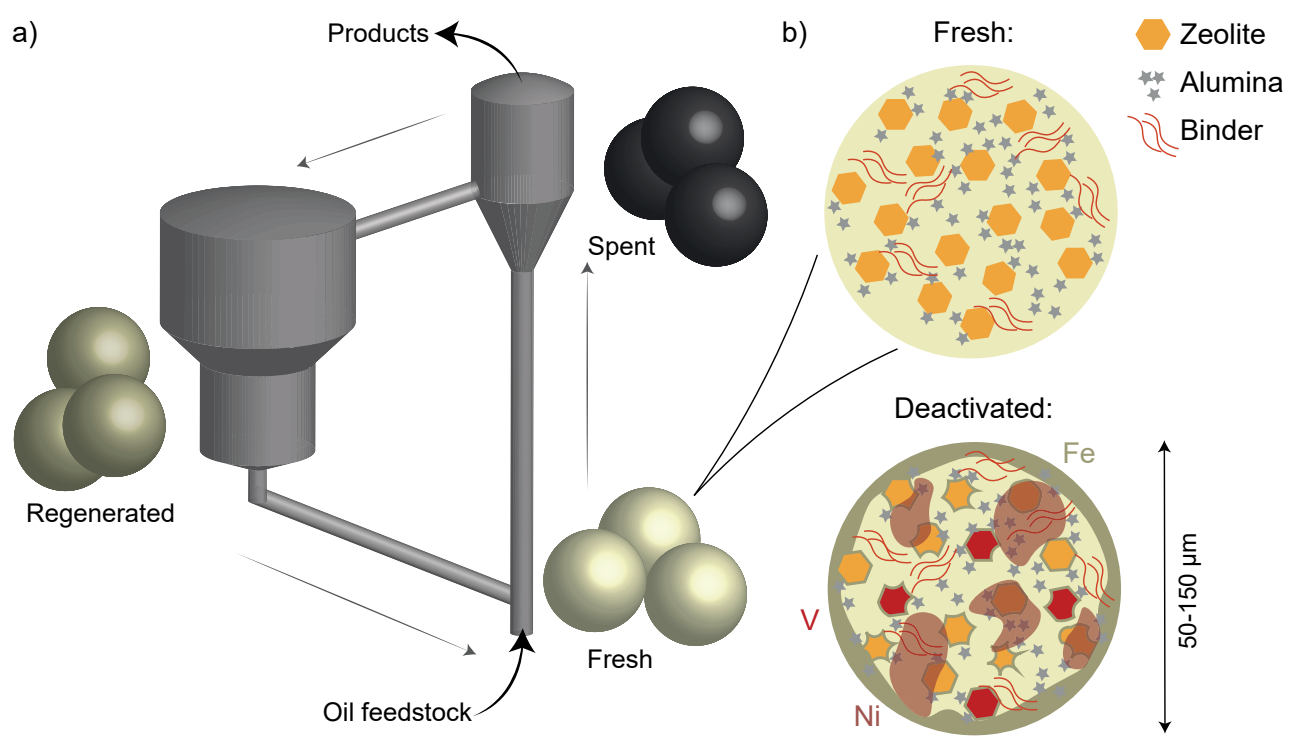

Figure 1.4: Schematic representation of the FCC process (a) and a detailed image of the multicomponent catalyst (b) that deactivates due to dealumination and the accumulation of metals.

zeolite dealumination, all occur simultaneously during the FCC process. Most studies, however, address only one of these processes, making use of lab-deactivated FCC catalyst samples. Separating these two effects makes their investigation easier: the influence of dealumination due to steaming or the accumulation of metals coming from the oil feedstock on the catalytic performance of the FCC catalyst have been thoroughly studied on both the single particle and the bulk level. ${ }^{[63,65,71]}$ Furthermore, as mentioned before, due to the mix of fresh and deactivated FCC particles, the actual ECAT has a large intrinsic interparticle heterogeneity. This complicates the study of these industrially used catalyst particles even more. ${ }^{[72]}$ To investigate their deactivation, FCC ECAT particles are often sorted based on their density by flotation, which is associated with metal loading and age. ${ }^{[73]}$ Although the flotation method gives a negative correlation between density and catalytic activity, it is a batch process that does not give insight in the individual role of the metal poisons that deactivate FCC particles. Therefore, there is a need for a new method, that is, a tool able to sort FCC ECAT particles by activity or just one of the metals that deactivates them.

\subsubsection{Metal-based Catalysis}

The most important metals for catalysis are the transition metals in groups VIII and I-B of the periodic table. The efficiency of a catalytic reaction performed with a metal catalyst depends on the balance between atomic adsorption and dissociative adsorption of the reactants. The metal used for a catalytic reaction is chosen based on the reaction of interest. For a reaction that requires dissociation of one or more reactants, a metal with high dissociative adsorption strengths is preferred, found on the top left of the d-block 
elements. Towards the middle and bottom left, the (dissociative) adsorption strengths change to moderate and weak. ${ }^{[3]}$

The catalytic reaction thus happens on the surface of the selected metal. Therefore, these metals are almost always used in the form of small nanoparticles, to increase the available surface area. Due to the low stability of a batch of nanoparticles, they are supported on a (chemically inert) material such as alumina, silica, titania or carbon. The dispersion of the nanoparticles over these supports is of vital importance for the catalyst's activity. However, the preparation of such catalysts has proven to be non-trivial. ${ }^{[74,75]}$ If heterogeneities arise among the particles in for example the loading of nanoparticles, the use of microfluidic screening and sorting can be of interest to narrow down the spread in catalyst loading. An example of a preparation that led to a large inter-particle heterogeneity has been described by Plessers et al. ${ }^{[5]}$, where the color deviations of silica particles show the differences in Ag loading per silica particle.

Supported metal catalysts are often used in gas phase reactions or multiphase reactions with both gases and liquids. For now, catalyst diagnostics with using droplet microfluidics is not suitable for gas phase reactions, due to the lack in stability of gas bubbles. ${ }^{[44]}$ This will prevent the particles from staying in the gas bubbles. However, in this thesis we explore the possibilities to perform a multiphase hydrogenation with $\mathrm{Pd} /$ $\mathrm{SiO}_{2}$ as catalyst in a microreactor.

\subsubsection{Hydrotalcites in Basic Catalysis}

A third catalyst that will be used in this $\mathrm{PhD}$ thesis is a hydrotalcite. Different from FCC particles or $\mathrm{Pd} / \mathrm{SiO}_{2}$ with the active sites dispersed throughout the particle as zeolite domains or Pd nanoparticles, hydrotalcite particles consist of one and the same material. No support is used and the whole particle could in principle be active as catalyst, which lowers their mechanical stability. One of the challenges for single particle diagnostics with droplet microfluidics, therefore, lies in keeping the particles intact during encapsulation. Hydrotalcites (HT) or Layered Double Hydroxides (LDH) are Mg-Al based anionic clays with a salt like structure that is complex. Brucite layers form one part of the crystal structure, the other part consists of hydrated anions in interlayer areas between these brucite-like layers. A schematic overview can be found in Figure 1.5. ${ }^{[76]}$

The strength and amount of the basic sites can be varied by changing the $\mathrm{Mg}$-Al ratio, where a ratio of 3:1 yields a high basic site density. It is shown in previous research that the strength of the basic sites as well as the surface area can also be increased by calcination at $400-450{ }^{\circ} \mathrm{C}$. Upon calcination, the interlayer ions are burned off and changes in the brucite layers yield tetrahedral $\mathrm{Al}$ and $\mathrm{O}^{2-}$ species at the surface. Since $\mathrm{O}^{2-}$ has a relatively higher base strength compared to the untreated $\mathrm{OH}^{-}$sites, a more basic catalyst is yielded. The basicity can be further increased via impregnation of the calcined hydrotalcite with salts like $\mathrm{KOH}$ or $\mathrm{KNO}_{3}$. The anions, $\mathrm{OH}^{-}$and $\mathrm{NO}_{3}{ }^{-}$in this case, are incorporated in the 


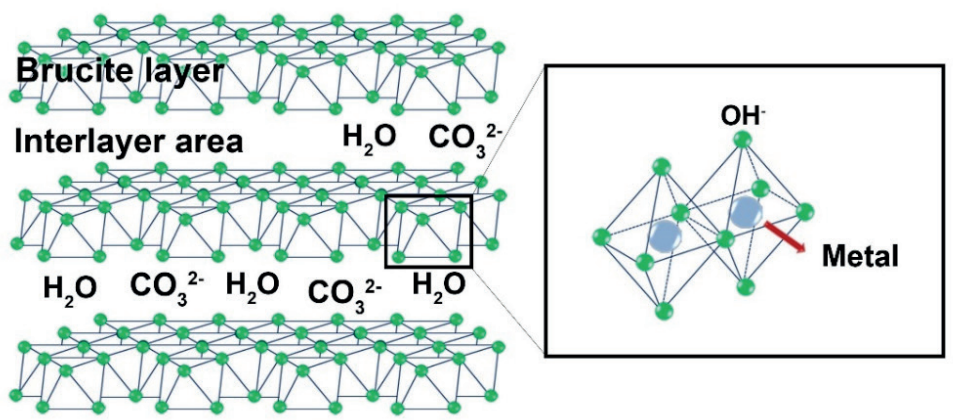

Figure 1.5: The molecular structure of a hydrotalcite with trivalent and divalent cations surrounded by hydroxide ions in brucite-like layers. Carbonate anions and water are positioned in the interlayer area. Adapted from [77].

interlayer areas, replacing water or $\mathrm{CO}_{3}{ }^{2-\left[{ }^{[78-80]}\right.}$

The use of different solvents also affects the catalytic performance of hydrotalcites in terms of their activity and selectivity. Aprotic polar solvents are reported with the best activity and selectivity. For non-polar solvents, the conversion is lower but a good selectivity is preserved. ${ }^{[79]}$ Hydrotalcites are known to be active heterogeneous base catalysts for $\mathrm{C}-\mathrm{C}$ bond formations or the transesterification of oils. Furthermore, they are used as supports for various metals that act in dehydrogenation and deoxygenation reactions. ${ }^{[81]}$

\subsection{Scope and Outline of this PhD Thesis}

In this $\mathrm{PhD}$ thesis, I will describe new results on the use of microfluidics for single catalyst particle diagnostics. The work will be divided in two parts, focused on (microfluidic) applications for Fluid Catalytic Cracking (FCC) single particle screening and on other heterogeneous catalytic systems. The former part focuses on acid-catalyzed reactions, and we make use of staining methods to visualize both catalyst acidity as well as accessibility. For the latter part, we have chosen metal-based catalysis with a multiphase hydrogenation reaction as well as a condensation reaction leading to the base-catalyzed formation of a fluorescent dye as showcase.

Chapter 2 of the PhD thesis involves single FCC ECAT particle sorting based on the particles' magnetic moment. A 3D-printed microreactor was used to sort FCC ECAT particles in five fractions with increasing Fe content and increasing amount of clustered Fe. Chapter 3 explains in more detail a multi-purpose method to easily screen many FCC ECAT particles for their metal content, acidity or porosity. A set of density separated FCC particles was analyzed with $\mu-\mathrm{XRF}$ and consecutive fluorescence microscopy, using probe molecules to correlate metals accumulation with both catalyst accessibility and acidity. For every individual FCC ECAT particle, the results of both techniques were correlated, resulting in more in-depth insights into all density separated fractions. In Chapter 4 and 5, we present two advanced microfluidic devices that use the same fluorescent 
probe molecules for a catalytic activity-based sorting of FCC ECAT particles. Using onchip heating, as described in Chapter 4, the oligomerization of 4-methoxystyrene was facilitated and using dielectrophoresis, the most active catalyst particles were sorted by separating them from the rest, as described in Chapter 5. A post analysis with $\mu$-XRF revealed that the highly fluorescent and thus most active FCC ECAT particles, contained lower amounts of Ni than the low/non-fluorescent FCC ECAT particles.

In the last two experimental chapters of the PhD thesis, other applications of droplet microfluidics for catalyst particle diagnostics are described. Chapter 6 describes a polydimethylsiloxane (PDMS) microreactor with permeable walls for diffusion of $\mathrm{H}_{2}$. The gas permeability through PDMS allows for performing a liquid phase hydrogenation of methylene blue inside droplets, having a single $\mathrm{Pd} / \mathrm{SiO}_{2}$ catalyst particle per droplet. In the last experimental chapter, Chapter 7, a tube-in-tube reactor based on fluorinated ethylene polymer (FEP) is used to catalyze the formation of a fluorescent coumarin, using a basic hydrotalcite material as catalyst. With this setup, we show that it is not always inevitable to use more expensive or time-consuming lithography-based fabrication techniques for droplet microfluidics. Also, we show the difficulties of working with mechanically unstable materials.

The $\mathrm{PhD}$ thesis will end with an overview of the main conclusions arising from this work, as well as an outlook on potential future research.

\subsection{References}

[1] M. Boudart, in Handb. Heterog. Catal. (Eds.: G. Ertl, H. Knözinger, J. Weitkamp), Wiley-VCH Weinheim, 2008.

[2] J. Hagen, Industrial Catalysis, Wiley-VCH, Weinheim, 2015.

[3] L. Lefferts, E. Hensen, H. Niemantsverdriet, in Catal. an Integr. Textb. Students (Eds.: U. Hanefeld, L. Lefferts), Wiley-VCH Weinheim, 2018, pp. 15-70.

[4] B. M. Weckhuysen, Angew. Chem. Int. Ed. 2009, 48, 4910-4943.

[5] E. Plessers, I. Stassen, S. P. Sree, K. P. F. Janssen, H. Yuan, J. Martens, J. Hofkens, D. De Vos, M. B. J. Roeffaers, ACS Catal. 2015, 5, 6690-6695.

[6] I. L. C. Buurmans, B. M. Weckhuysen, Nat. Chem. 2012, 4, 873-886.

[7] M. Sivaramakrishnan, R. Kothandan, D. K. Govindarajan, Y. Meganathan, K. Kandaswamy, Curr. Opin. Biomed. Eng. 2020, 13, 60-68.

[8] A. Manz, N. Graber, H. M. Widmers, Sensors Actuators B Chem. 1990, 1, 244-248.

[9] G. M. Whitesides, Nature 2006, 442, 368-373.

[10] A. A. S. Bhagat, H. Bow, H. W. Hou, S. J. Tan, J. Han, C. T. Lim, Med. Biol. Eng. Comput. 2010, 48, 999-1014.

[11] W. C. Shields IV, C. D. Reyes, G. P. López, Lab Chip 2015, 15, 1230-1249.

[12] D. R. Gossett, W. M. Weaver, A. J. MacH, S. C. Hur, H. T. K. Tse, W. Lee, H. Amini, D. Di Carlo, Anal. Bioanal. Chem. 2010, 397, 3249-3267.

[13] A. Valero, J. N. Post, J. W. Van Nieuwkasteele, P. M. Braak, W. Kruijer, A. Van Den Berg, Lab Chip 2008, 8, 62-67.

[14] E. Y. Basova, F. Foret, Analyst 2015, 140, 22-38.

[15] R. M. Tiggelaar, F. Benito-l, D. C. Hermes, H. Rathgen, R. J. M. Egberink, F. G. Mugele, D. N. Reinhoudt, A. Van Den Berg, W. Verboom, H. J. G. E. Gardeniers, Chem. Eng. J. 2007, 131, 163-170.

[16] P. Van Male, M. H. J. M. De Croon, R. M. Tiggelaar, A. Van Den Berg, J. C. Schouten, Int. J. Heat Mass Transf. 2004, 47, 87-99.

[17] G. N. Doku, W. Verboom, N. Reinhoudt, A. Van Den Berg, Tetrahedron 2005, 61, 2733-2742.

[18] H. Wensink, F. Benito-lopez, D. C. Hermes, W. Verboom, H. J. G. E. Gardeniers, N. Reinhoudt, A. Van Den 
Berg, Lab Chip 2005, 5, 280-284.

[19] S. Marre, K. F. Jensen, Chem. Soc. Rev. 2010, 39, 1183-1202.

[20] M. Tokeshi, T. Minagawa, K. Uchiyama, A. Hibara, K. Sato, H. Hisamoto, T. Kitamori, Anal. Chem. 2002, 74, 1565-1571.

[21] T.-A. Meier, R. J. Beulig, E. Klinge, M. Fuss, S. Ohla, D. Belder, Chem. Commun. 2015, 51, 8588-8591.

[22] K. L. A. Chan, S. Gulati, J. B. Edel, A. J. de Mello, S. G. Kazarian, Lab Chip 2009, 9, 2909-2913.

[23] Y. H. Kim, L. Zhang, T. Yu, M. Jin, D. Qin, Y. Xia, Small 2013, 9, 3462-3467.

[24] X. Yao, Y. Zhang, L. Du, J. Liu, J. Yao, Renew. Sustain. Energy Rev. 2015, 47, 519-539.

[25] W. E. Svendsen, in Lab-on-a-Chip Devices Micro-Total Anal. Syst. (Eds.: J. Castillo-león, W.E. Svendsen), Springer Berlin, 2015, pp. 17-26.

[26] S. Colin, in Microfluidics (Ed.: S. Colin), John Wiley And Sons New York, 2010, pp. 1-23.

[27] A. D. Stroock, S. K. W. Dertinger, A. Ajdari, I. Mezi , H. A. Stone, G. M. Whitesides, Science (80-. ). 2002, 295, 647-651.

[28] S. Zeng, X. Liu, H. Xie, B. Lin, in Top. Curr. Chem. (Ed.: B. Lin), Springer, Berlin, Heidelberg, 2011, pp. 69-90.

[29] Z. Z. Chong, S. H. Tan, A. M. Gañán-calvo, S. Tor, N. H. Loh, N. Nguyen, Lab Chip 2016, 16, $35-58$.

[30] P. Zhu, L. Wang, Lab Chip 2017, 17, 34-75.

[31] R. M. Schoeman, E. W. M. Kemma, F. Wolbers, A. van den Berg, Electrophoresis 2014, 35, 385-392.

[32] D. J. Collins, A. Neild, A. DeMello, A. Liu, Y. Ai, Lab Chip 2015, 15, 3439-3459.

[33] T. P. Lagus, J. F. Edd, J. Phys. D. Appl. Phys. 2013, 46, 114005.

[34] N. Nivedita, P. Ligrani, I. Papautsky, Sci. Rep. 2017, 7, 44072.

[35] J. F. Edd, D. Di Carlo, K. J. Humphry, S. Köster, D. Irimia, D. A. Weitz, M. Toner, Lab Chip 2008, 8, $1262-1264$.

[36] B. Kuswandi, Nuriman, J. Huskens, W. Verboom, Anal. Chim. Acta 2007, 601, 141-155.

[37] Q. Zhou, T. Kim, Sensors Actuators B. Chem. 2016, 227, 504-514.

[38] A. März, T. Henkel, D. Cialla, M. Schmitt, J. Popp, Lab Chip 2011, 11, 3584.

[39] T. Hartman, C. S. Wondergem, N. Kumar, A. Van Den Berg, B. M. Weckhuysen, J. Phys. Chem. Lett. 2016, 7, 1570-1584.

[40] “https://www.hamamatsu.com/eu/en/product/optical-sensors/pmt/about_pmts/index.html," n.d.

[41] A. Autour, M. Ryckelynck, Micromachines 2017, 8, 128-149.

[42] S. Sukas, E. Schreuder, B. De Wagenaar, J. Swennenhuis, A. Van Den Berg, L. Terstappen, S. Le Gac, Lab Chip 2014, 14, 1821-1825.

[43] H. Morgan, D. Holmes, N. G. Green, Curr. Appl. Phys. 2006, 6, 367-370.

[44] J. Yue, Catal. Today 2018, 308, 3-19.

[45] L. N. Protasova, E. V. Rebrov, H. E. Skelton, A. E. H. Wheatley, J. C. Schouten, Appl. Catal. A Gen. 2011, 399, 12-21.

[46] N. Cherkasov, A. O. Ibhadon, E. V. Rebrov, Lab Chip 2015, 15, 1952-1960.

[47] E. V. Rebrov, A. Berenguer-Murcia, H. E. Skelton, B. F. G. Johnson, A. E. H. Wheatley, J. C. Schouten, Lab Chip 2009, 9, 503-506.

[48] I. Lignos, S. Stavrakis, A. Kilaj, A. J. deMello, Small 2015, 11, 4009-4017.

[49] M. A. Levenstein, C. Anduix-Canto, Y. Y. Kim, M. A. Holden, C. González Niño, D. C. Green, S. E. Foster, A. N. Kulak, L. Govada, N. E. Chayen, S. J. Day, C. C. Tang, B. Weinhausen, M. Burghammer, N. Kapur, F. C. Meldrum, Adv. Funct. Mater. 2019, 29, 1-12.

[50] H.-D. Xi, H. Zheng, W. Guo, A. M. Ganan-Calvo, Y. Ai, C.-W. Tsao, J. Zhou, W. Li, Y. Huang, N.-T. Nguyen, S. H. Tan, Lab Chip 2017, 17, 751-771.

[51] P. R. C. Gascoyne, J. Vykoukal, Electrophoresis 2002, 23, 1973-1983.

[52] T. Sun, H. Morgan, in Electrokinet. Electrohydrodynamics Microsystems (Ed.: A. Ramos), Springer, New York, 2011, pp. 1-28.

[53] I. Chorkendorff, J. W. Niemantsverdriet, in Concepts Mod. Catal. Kinet., Wiley VHC, 2003, pp. 167-214.

[54] H. S. Cerqueira, G. Caeiro, L. Costa, F. R. Ribeiro, J. Mol. Catal. A Chem. 2008, 292, 1-13.

[55] E. T. C. Vogt, B. M. Weckhuysen, Chem. Soc. Rev. 2015, 44, 7342-7370.

[56] "A National Historic Chemical Landmark: The Houdry Process," 1996.

[57] W. Letzsch, in Handb. Pet. Process. (Ed.: S.A. Treese), Springer, Cham, 2015, pp. 262-312.

[58] R. Sadeghbeigi, Fluid Catalytic Cracking Handbook. An Expert Guide to the Practical Operation, Design and Optimization of FCC Units., Elsevier, Amsterdam, 2012. 
[59] J. Biswas, I. E. Maxwell, Appl. Catal. 1990, 63, 197-258.

[60] V. Komvokis, L. X. L. Tan, M. Clough, S. S. Pan, B. Yilmaz, in Zeolites Sustain. Chem. (Eds.: F.-S. Xiao, X. Meng), Springer, Cham, 2016, pp. 271-297.

[61] A. M. Wise, J. N. Weker, S. Kalirai, M. Farmand, D. A. Shapiro, F. Meirer, B. M. Weckhuysen, ACS Catal. 2016, 6, 2178-2181.

[62] D. A. M. de Winter, F. Meirer, B. M. Weckhuysen, ACS Catal. 2016, 6, 3158-3167.

[63] B. I. R. Mitchell, Ind. Eng. Chem. Prod. Res. Dev. 1980, 19, 209-213.

[64] F. Meirer, S. Kalirai, D. Morris, S. Soparawalla, Y. Liu, G. Mesu, J. C. Andrews, B. M. Weckhuysen, Sci. Adv. 2015, 1, el400199.

[65] F. Meirer, D. T. Morris, S. Kalirai, Y. Liu, J. C. Andrews, B. M. Weckhuysen, J. Am. Chem. Soc. 2015, 137, 102-105.

[66] F. Meirer, S. Kalirai, J. Nelson Weker, Y. Liu, J. C. Andrews, B. M. Weckhuysen, J. Scherzer, I. L. C. Buurmans, B. M. Weckhuysen, Chem. Commun. 2015, 51, 8097-8100.

[67] S. Kalirai, U. Boesenberg, G. Falkenberg, F. Meirer, B. M. Weckhuysen, ChemCatChem 2015, 7, 36743682 .

[68] Y. Liu, F. Meirer, C. M. Krest, S. Webb, B. M. Weckhuysen, Nat. Commun. 2016, 7, 12634.

[69] J. Ihli, D. Ferreira Sanchez, J. Rosh, V. Cuartero, O. O. Mathon, F. Krumeich, C. Borca, T. Huthwelker, W.-C. C. Cheng, Y.-Y. Y. Shu, S. Pascarelli, D. Grolimund, A. Menzel, J. A. van Bokhoven, Angew. Chem. Int. Ed. 2017, 56, 14031-14035.

[70] J. Ihli, R. R. Jacob, M. Holler, M. Guizar-Sicairos, A. Diaz, J. C. Da Silva, D. Ferreira Sanchez, F. Krumeich, D. Grolimund, M. Taddei, W. C. Cheng, Y. Shu, A. Menzel, J. A. Van Bokhoven, Nat. Commun. 2017, 8, 809.

[71] I. L. C. Buurmans, J. Ruiz-Martínez, W. V Knowles, D. Van Der Beek, J. A. Bergwerff, E. T. C. Vogt, B. M. Weckhuysen, Nat. Chem. 2011, 3, 862-867.

[72] M. A. Karreman, I. L. C. Buurmans, A. V Agronskaia, J. W. Geus, H. C. Gerritsen, B. M. Weckhuysen, Chem. Eur. J. 2013, 19, 3846-3859.

[73] G. R. Dyrkacz, L. Ruscic, C. L. Marshall, W. Reagan, Energy \& Fuels 2000, 71, 849-854.

[74] W. S. Lamme, J. Ze evi , K. P. de Jong, ChemCatChem 2018, 10, 1552-1555.

[75] P. Munnik, N. A. Krans, P. E. De Jongh, K. P. De Jong, ACS Catal. 2014, 4, 3219-3226.

[76] W. Bing, M. Wei, J. Solid State Chem. 2019, 269, 184-194.

[77] L. P. Figueredo Benício, R. Alvarenga Silva, J. Aparecida Lopes, D. Eulálio, R. M. M. dos Santos, L. A. De Aquino, L. Vergütz, R. Ferreira Novais, L. M. Da Costa, F. Garcia Pinto, J. Tronto, Rev. Bras. Cienc. do Solo 2015, 39, 1-13.

[78] J. I. Di Cosimo, V. K. Diez, M. Xu, E. Iglesia, C. R. Apesteguía, J. Catal. 1998, 178, 499-510.

[79] R. Devi, P. Begum, P. Bharali, R. C. Deka, ACS Omega 2018, 3, 7086-7095.

[80] S. V. Cherepanova, N. N. Leont'Eva, A. B. Arbuzov, V. A. Drozdov, O. B. Belskaya, N. V. Antonicheva, J. Solid State Chem. 2015, 225, 417-426.

[81] . Nishimura, A. Takagaki, K. Ebitani, Green Chem. 2013, 15, 2026-2042. 


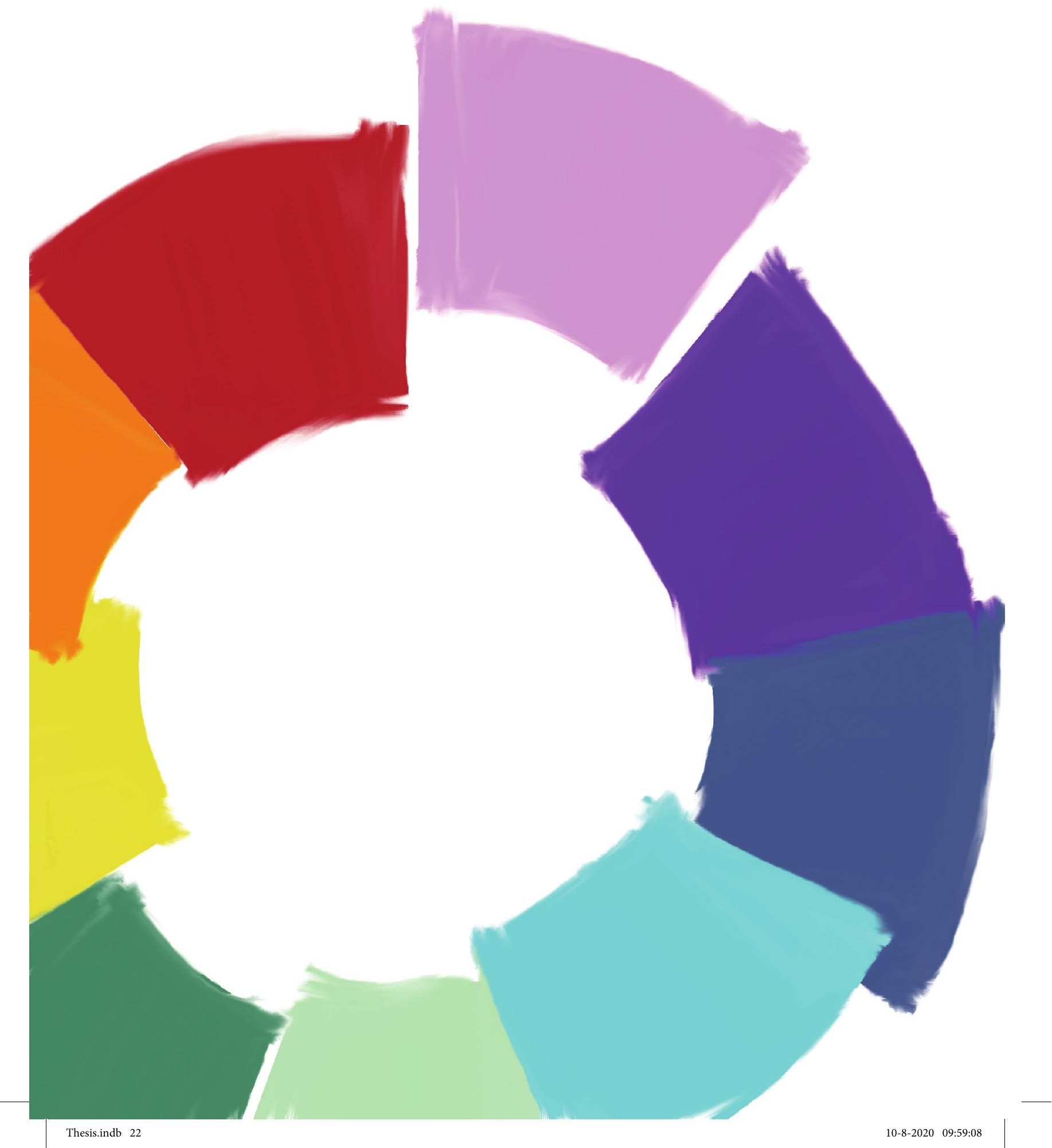




\title{
Chapter 2
}

\section{Magnetophoretic Sorting of Single Fluid Catalytic Cracking Catalyst Particles}

\begin{abstract}
A better understanding of the deactivation processes taking place within solid catalysts is vital to design better catalysts. Since inter-particle heterogeneities are more rule than exception, particle sorting is crucial to analyze single catalyst particles in more detail. Microfluidics offers new possibilities to sort catalysts at the single particle level in a high-throughput fashion. In this Chapter, we report a first-of-its-kind 3D-printed magnetophoretic chip able to sort catalyst particles by their magnetic moment. Fluid Catalytic Cracking (FCC) particles were separated based on their Fe content, one of the deactivating metals. With magnetophoretic sorting, we show that large Fe aggregates exist within $20 \%$ of the FCC particles with the highest Fe content. Magnetic properties point into the direction that differences in magnetic moment are caused by an increased number of Fe nano-clusters with equal sizes. Furthermore, it was found that the availability of Brønsted acid sites within FCC particles decreases with increasing Fe content. This work paves the way towards a high-throughput catalyst diagnostics platform to determine why specific catalyst particles perform better than others
\end{abstract}

This work is based on the following manuscript: M. Solsona*, A.-E. Nieuwelink*, F. Meirer, L. Abelmann, M. Odijk, W. Olthuis, B.M. Weckhuysen, A. van den Berg, Magnetophoretic Sorting of Single Catalyst Particles, Angew. Chem. Int. Ed. 2018, 57, 10589-10594.

*These authors contributed equally. 


\subsection{Introduction}

To sort a heterogeneous set of Fluid Catalytic Cracking (FCC) catalyst particles in batches with less inter-particle heterogeneities, in this Chapter we present a 3D-printed magnetophoretic chip. ${ }^{[1]}$ This chip, schematically outlined in Figure 2.1a, is able to sort individual catalyst particles by their magnetic moment. The sorted FCC particles were subsequently analyzed by X-ray fluorescence (XRF) microscopy (Figure 2.1c), fluorescence micro-spectroscopy (Figure 2.1d), magnetic hysteresis, and field cooled magnetic measurements (Figure 2.1b) to explain the origin of the sorting principle. Magnetophoresis can be used to sort magnetic from non-magnetic particles and fluids (ferrofluids) ${ }^{[2]}$, and has previously been used to sort, for example, cells and magnetic particles. $^{[3-8]}$ This very reproducible approach could also be used for other magnetic catalysts, containing e.g. Ni or Co.

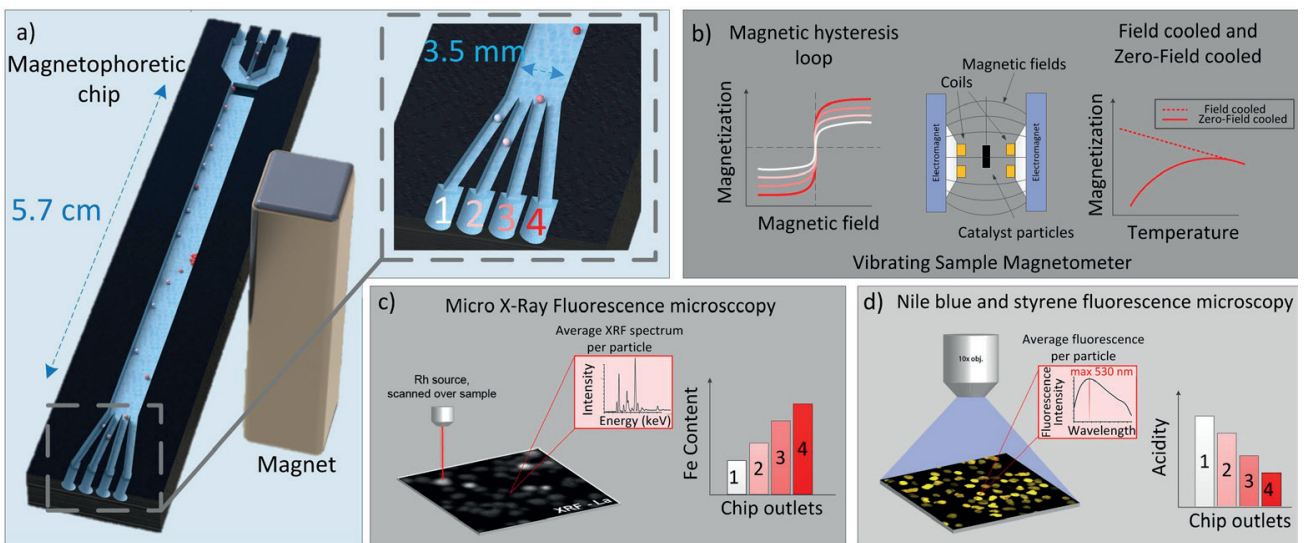

Figure 2.1: (a) Sketch of the inside channel of the 3D-printed microfluidic chip for sorting catalyst particles. (b) Sketch of the vibrating sample magnetometer, inverted hysteresis loops of the different outlets and zero-field cooled and field cooled measurements. (c) micro X-ray fluorescence and (d) fluorescence microspectroscopy to analyse the sorted FCC catalyst particles.

Previous work on FCC materials has found (at least) two types of Fe distributed within catalyst particles: Fe inside the clay components of the catalyst and Fe deposited during the FCC process, which is mainly accumulated in the first 2-3 $\mu \mathrm{m}$ of the outer surface of the particle. This deposited $\mathrm{Fe}$, which originates from the crude oil feedstock or from reactor debris, is mainly held responsible for the deactivation of these catalysts due to pore blocking. ${ }^{[9-12]}$ Furthermore, it was shown that these two types of Fe distributions have different oxidation states. Inside the clay, $\mathrm{Fe}$ is found as $\mathrm{Fe}^{0}$ and as $\mathrm{Fe}^{\mathrm{II}}$ in the form of $\mathrm{FeO}$ and $\mathrm{SiFeO}_{3}{ }^{[12]}$ On the other hand, $\mathrm{Fe}^{\mathrm{III}}$ is found on/in the outer FCC particle layer. ${ }^{[10]}$

Equilibrium FCC particles, better known as ECAT particles, were previously sorted by magnetic fields in order to decrease the disposal of spent catalyst in FCC reactors. This industrial approach consisted of a permanent rolling magnet cylinder under a belt where particles with different sizes were fed on, to sort them in just two fractions. ${ }^{[13]}$ In this Chapter, however, the FCC particles have been pre-sorted by size and we focus on establishing a relation between the Fe content, magnetic properties and the availability of 
Brønsted acid sites ${ }^{[14-17]}$ in a wide range of Fe content and on real, industrially processed ECAT particles, providing a unique catalyst diagnostics platform for single particle analysis.

\subsection{Experimental Section}

\subsubsection{Sorting of Catalyst Particles}

A 3D-printed chip was designed in SolidWorks ${ }^{\circledR}$ and printed with a Formlabs ${ }^{\circledR}$ Form 2 printer using clear resin. A chip holder was fabricated with black delrin (polyacetal) and brass; and consists of a fixed and mobile part where the microfluidic chip and the magnet were fixated, respectively, as shown in Figure 2.2.

The ECAT particles were sorted with sieves $(70-90 \mu \mathrm{m})$ and calcined at $600{ }^{\circ} \mathrm{C}\left(1^{\circ} \mathrm{C} /\right.$ min to $120^{\circ} \mathrm{C}$ followed by $5^{\circ} \mathrm{C} / \mathrm{min}$ ) to remove all remaining coke deposits that could be present on the catalyst particles. To adapt the viscosity and thereby the residence time of

a)
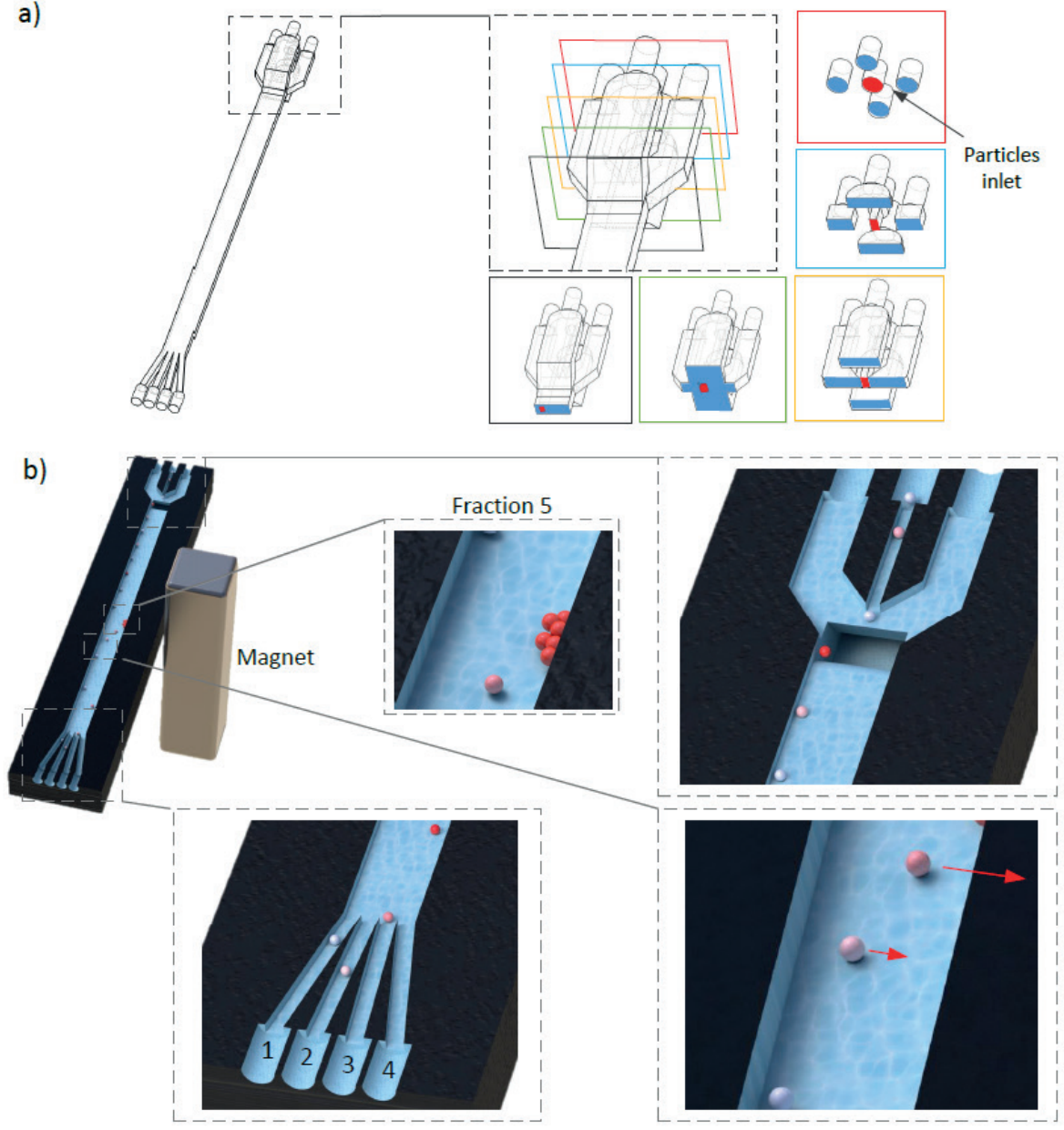

Figure 2.2: (a) Schematic of the liquid (blue) and particle (red) inlet and flow focusing inside the channel and (b) catalyst particles with a larger magnetic moment end up in a channel closer to the magnet or stick to the channel wall as fraction 5 . 
the particles in the magnetic field, calcined ECAT particles were introduced in a mixture of glycerol and water 1:3 vol., in an open vertical syringe and stirred to avoid excessive sedimentation. A 1 x 1 x $4 \mathrm{~cm}$ Neodymium magnet with a remanence of $1.23 \mathrm{~T}$ purchased from Supermagnete ${ }^{\circledR}$ was fixed in the chip holder's mobile part and approached to the chip at a known distance of $4 \mathrm{~cm}(\mathrm{Br}=0.08 \mathrm{~T})$. Four $50 \mathrm{~mL}$ syringes were used with three Nemesys ${ }^{\circledR}$ BASE 120 and one Harvard apparatus PHD 2000 programmable syringe pumps with flows of 120, 120, 30 and $800 \mu \mathrm{l} / \mathrm{min}$. A Grasshopper ${ }^{\circledR}{ }^{\circledR}$ GS3-U3-23S6M highspeed camera was used to record the trajectories of the ECAT particles inside the chip. Stills from the movies recorded at the top of the microchannel can be seen in Figure 2.3d, were the ECAT particles (indicated with white circles) clearly follow the same trajectory before they reach the magnet.

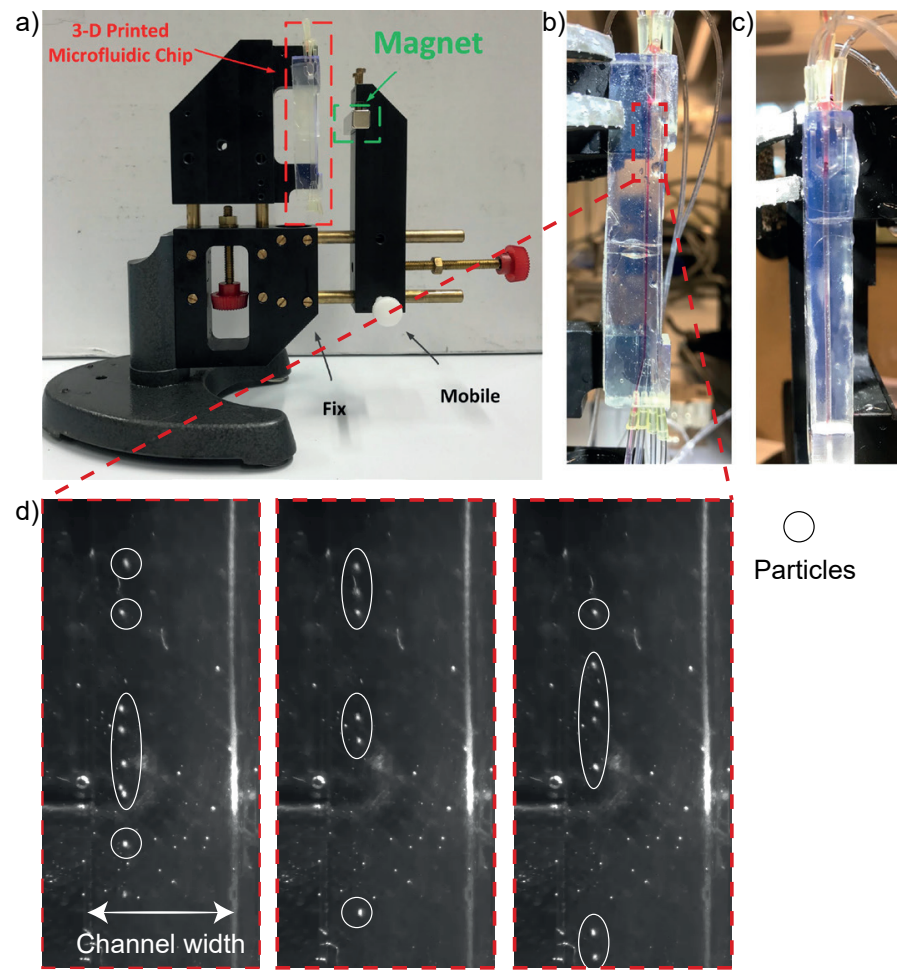

Figure 2.3: Photographs of (a) the chip holder, 3D-microfluidic chip with magnet, (b) front view and (c) side view of the $2 \mathrm{D}$ flow focusing of dye in 3D-microfluidic chip. In (d) stills from the recorded movies show particles at the top of the microchannel.

\subsubsection{Characterization of Sorted Fractions of Catalyst Particles}

A small portion of the sorted ECAT catalyst particles was placed between two $5 \times 5$ mm tape layers and introduced in a Quantum Design ${ }^{\circledR}$ PPMS-VSM (which stands for Physical Property Measurement System Vibrating Sample Magnetometer) to measure their magnetic moment. The scan rate was $0.02 \mathrm{~T}$ per second from $9 \mathrm{~T}$ to $-9 \mathrm{~T}$ and up to $9 \mathrm{~T}$ again at room temperature. The diamagnetic contribution was extracted from 
the samples until a plateau at high magnetic fields was observed. For Scanning Electron Microscopy - Energy Dispersive X-ray analysis (SEM-EDX), the particles were embedded in a polymer resin and sliced to image the interior of the particles. For Field Cooled/Zero Field Cooled (FC/ZFC) measurements, the sorted ECAT particles were introduced in Ge Varnish mounted on a $5 \times 5 \mathrm{~mm}$ silicon holder. Afterwards, the sample was introduced in the Quantum Design ${ }^{\circledR}$ PPMS-VSM. ZFC: The sample was cooled down to $10 \mathrm{~K}$ at $0 \mathrm{~T}$ and heated up to $305 \mathrm{~K}$ at $0.05 \mathrm{~T}$. FC: a magnetic field of $0.05 \mathrm{~T}$ was applied, and the sample was cooled down until $10 \mathrm{~K}$. Then, the magnetic field was set to $0 \mathrm{~T}$ and the samples were heated up until $305 \mathrm{~K}$. The FC/ZFC, PPMS-VSM and SEM-EDX measurements were performed at the MESA+ Nanolab of the University of Twente. For the electron paramagnetic resonance (EPR) measurements, dried and calcined particles of fraction F5 were measured with a Bruker EMX Plus spectrometer operating with an ER 041 XG X-band microwave bridge and operating at a frequency of $\sim 9.5 \mathrm{GHz}$. For the UV-vis microscopy spectra, ECAT particles from fraction F5 with visible clusters were selected. With a CRAIC 20/30 PVTM micro-spectrophotometer in reflectance mode, equipped with a $74 x 0.65$ NA reflective lens and $30 \mathrm{~W}$ halogen lamp, UV-vis spectra were collected from a spot indicated with a black square in the images.

For the acidity and metal loading characterization of the catalyst particles, the magnetically sorted ECAT fractions were carefully dried and calcined in air by placing them in a ceramic crucible. The samples were heated to $120^{\circ} \mathrm{C}\left(\mathrm{ramp} 1{ }^{\circ} \mathrm{C} / \mathrm{min}\right)$ for $1 \mathrm{~h}$ to remove water and then heated to $600^{\circ} \mathrm{C}\left(\operatorname{ramp~} 5^{\circ} \mathrm{C} / \mathrm{min}\right.$ ) for $5 \mathrm{~h}$. For the $\mathrm{Br} \phi$ nsted acidity measurements, the separated fractions of catalyst particles were placed on sticky tape. A droplet of 4-methoxystyrene was placed next to the tape and the sample was heated to $100{ }^{\circ} \mathrm{C}$ for $15 \mathrm{~min}$. The sample was placed in a closed environment by covering the sample with a petri dish. The 4-methoxystyrene (Sigma Aldrich, 97\%) could enter the pores of the FCC particles via gas diffusion. With a Nikon Al confocal fluorescence microscope (10x objective) equipped with a $488 \mathrm{~nm}$ laser, the fluorescence intensity of the FCC ECAT particles was measured. Details of these staining experiments can be found in the literature. ${ }^{[17,18]}$ For pore accessibility measurements, the separated ECAT fractions were added to a solution of $0.1 \mathrm{wt} \%$ Nile blue A (Acros Organics, pure) in ethanol (Sigma Aldrich, 100\%). After equilibrating for $1 \mathrm{~h}$, the catalyst particles were placed on sticky tape and analyzed with the same confocal fluorescence microscope using a $561 \mathrm{~nm}$ laser.

The different fractions of sorted ECAT catalyst particles were also characterized with micro-X-ray fluorescence ( $\mu$-XRF) to determine the presence of metals in the catalyst particles. For the $\mu-\mathrm{XRF}$ measurements, the samples that were used for the 4-methoxystyrene staining were dried carefully and mounted in an Orbis PC SDD with a Rh-tube as X-ray source ( $30 \mathrm{kV}$ and $200 \mathrm{nA}$ ). With a spot size of $30 \mu \mathrm{m}$ and a step size of $15 \mu \mathrm{m}$ the surface was scanned (300 ms integration time) to obtain an XRF map for each element with per pixel the highest intensity for the element shown in the map.

\subsection{Results and Discussion}

The developed 3D-printed microfluidic chip consists of 5 inlets ( 4 inlets to focus the 
catalyst particles inside the chip and 1 inlet to introduce the catalyst particles), a straight channel of $5.7 \mathrm{~cm}$ long, $3.5 \mathrm{~mm}$ wide and $1 \mathrm{~mm}$ deep where the particles get attracted to the magnet, and 4 outlets (Figure 2.2). Considering the purpose of the device to sort particles by their Fe content, and knowing that the magnetic force is related to the total mass of Fe in the particle, the ECAT particles were size pre-sorted with two sieves in a range of 70-90 $\mu \mathrm{m}$. In this way, we can roughly say that particles with a similar Fe concentration end up in the same sorted fraction and bigger particles with similar Fe concentrations but naturally a higher total mass of Fe cannot interfere. Based on the large differences between magnetic moments found for the ECAT particles, they were sorted into 5 fractions; four microfluidic outlets and a fifth fraction formed by the particles that adhered to the channel wall at the position of the magnet (Figure 2.2b, fraction 5).

To know the magnetic properties of the sorted FCC particles, their magnetic moment was measured, and the results are shown in Figure 2.4a. Hysteresis loops per fraction F1 to F5 and averaged per single ECAT particle show a superparamagnetic behavior, no significant coercivity and an increasing moment per particle with increasing fraction number. This is an indication for either an increase in the amount of magnetic material inside the catalyst particles and/or a change in Fe-oxide form or oxidation state of certain magnetic materials. Consequently, $\mu$-XRF measurements were performed by mapping the elemental composition of the ECAT particles in all sorted fractions. Figure $2.4 \mathrm{~d}$ gives the average intensity of all elements that were detected and Figure $2.4 \mathrm{~b}$ shows the most important magnetic elements $\mathrm{Fe}$ an Ni that were found in the $\mu-\mathrm{XRF}$ maps. Due to the absence of reference samples (read, fresh FCC particles) with known Fe concentrations, and the data analysis without peak fitting of the $\mu$-XRF measurements,

a)

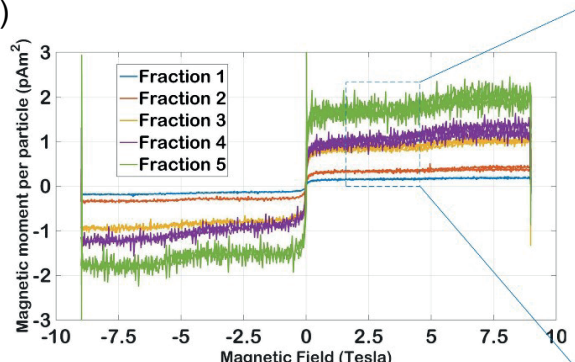

b)

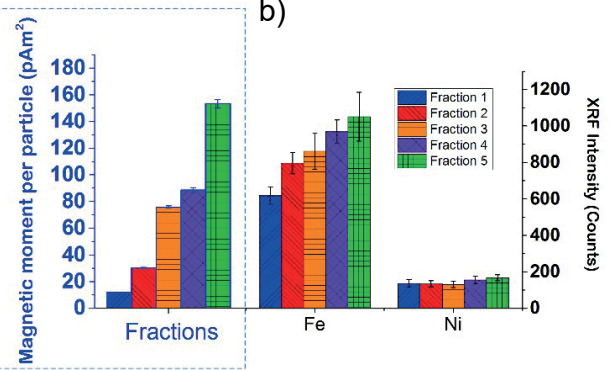

d)

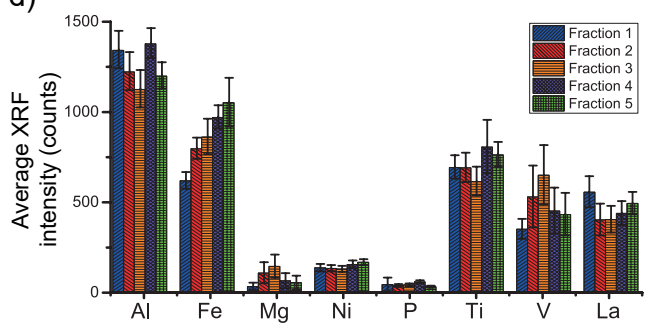

Figure 2.4: (a) Averaged hysteresis loops of the sorted ECAT particles per outlet and per particle. Number of particles per outlet: $1=466,2=255,3=120,4=80$ and $5=44$, (b) (right axis) relative XRF Fe and Ni intensities and (left axis) averaged magnetic moment per fraction of the sorted single ECAT particle. From the particles' diameter, it was confirmed that sorting was not based on size (c). All elements measured with $\mu$-XRF are plotted in (d). The error bars correspond to the standard deviation that rises from averaging the XRF values of multiple particles in the $\mu \mathrm{XRF}$ map. 
no real quantitative results can be obtained from these plots. However, trends between the fractions can be observed. For example, the La content is very similar in all fractions; La is used as stabilizing ion in zeolite US-Y and can, therefore, be used as an indication of the amount of US-Y zeolite within the FCC ECAT particles. ${ }^{[19]}$ In our case, all sorted fractions have a similar zeolite content. An increasing average Fe content in the ECAT particles from fraction F1 to F5 was found, confirming that Fe is the main actor on the magnetic attraction to the magnet. Also, when Fe increases by a factor of roughly 2 , the magnetic moment increases by a factor of 13. This seems to point to a change in Feoxide form and/or oxidation state. In FCC deactivation research, $\mathrm{Ni}$ is typically used as a measure for the catalytic age of an FCC particle, since its concentration increases linearly with deactivation of the FCC particles and is, in contrast to Fe, not present in fresh FCC particles. ${ }^{[20-22]}$ However, the ECAT under study has a low Ni content, therefore there are no clear trends observed that link our magnetophoretic sorting to particle catalytic age. The average particle diameter as shown in Figure $2.4 \mathrm{c}$ is similar for all fractions, confirming that the magnetophoretic sorting was not based on size.

When taking a closer look at the FCC particles in the five fractions, aggregates were observed on the surface of the particles of fractions F2 to F5. Scanning electron microscopy-energy dispersive X-ray (SEM-EDX) analysis, electron paramagnetic resonance (EPR) and UV-vis microscopy measurements revealed that both black and orange aggregates consist of iron oxide. For the SEM-EDX measurements shown in Figure 2.5a, a single particle was embedded in a resin that was diced until the black spot was exposed. EDX elemental mapping shows that the cluster, appearing black on the optical image on the left, shows a high Fe content. At the same time, the Al concentration at this point is lower, while $\mathrm{Si}$ and $\mathrm{O}$ show similar concentrations compared to the rest of the particle. Due to the fact that the black spot couldn't be measured directly from the surface, we can conclude that it was deposited under a layer of a few micrometers thick. From the EPR data, shown in Figure 2.5b, a broad single band with g-factor of $\sim 2.25$ is observed, indicating the presence of $\mathrm{Fe}_{\mathrm{X}} \mathrm{O}_{\mathrm{Y}}$ in the sample. The absence of an EPR signal with a g-factor of -3.0 points towards the absence of $\mathrm{Fe}^{\mathrm{II}}$ clusters in our sample. The g-value was calculated with the following Equation (Eq. 2.1 ${ }^{[23]}$ :

$$
\mathrm{g}=\mathrm{hv} /\left(\mu_{\mathrm{B}} \mathrm{B}_{0}\right) \text {, }
$$

with $h$ as Planck's constant, $v$ is the microwave frequency used (in $\mathrm{MHz}$ ), $\mu_{\mathrm{B}}$ is the Bohr magneton and $\mathrm{B}_{0}$ is the magnetic field peak center position. However, EPR is a bulk characterization method and therefore cannot give information solely on the surface aggregates. Therefore, UV-vis microscopy measurements were performed on several clusters to give more insight in their chemical composition. The UV-vis spectra, given in Figure 2.5c, show features corresponding to both $\alpha-\mathrm{Fe}_{2} \mathrm{O}_{3}$ and $\mathrm{FeO}(\mathrm{OH})$ : both are $\mathrm{Fe}$ structures with yellow-orange to red-brown colors. The color of an iron(III)oxide is due to the position of the EPT band (electron pair transition) around 480-530 nm. These ligand field $\mathrm{d}-\mathrm{d}$ transitions are spin forbidden, but have a high intensity due to magnetic coupling of the next to nearest neighbor $\mathrm{Fe}^{\mathrm{III}}$.24] 
a)

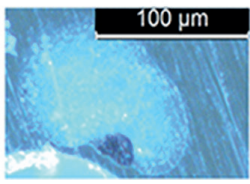

b)

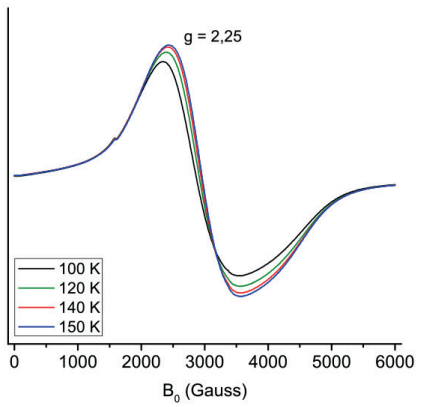

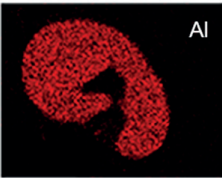
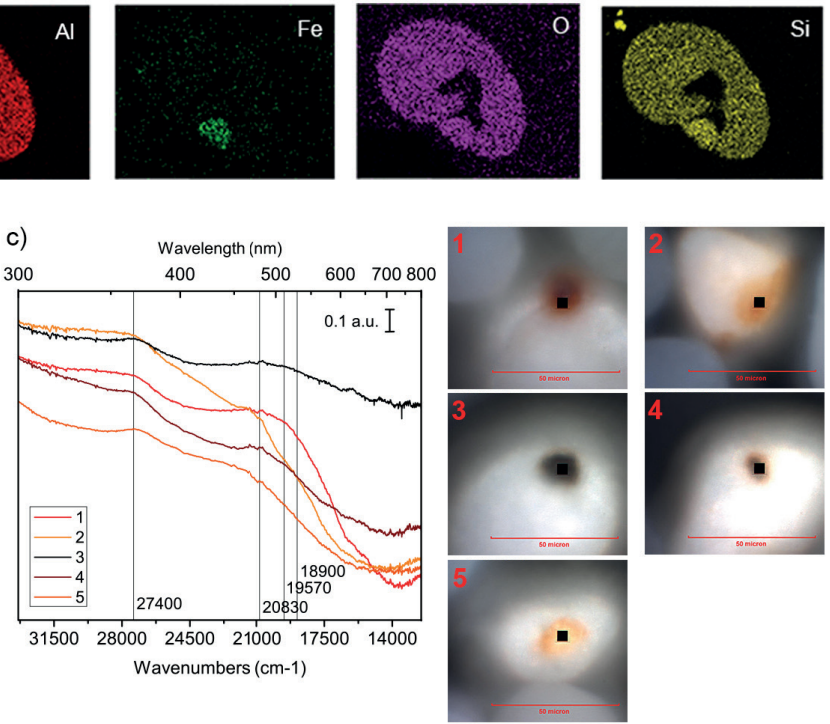

Figure 2.5: (a) Scanning electron microscopy-energy dispersive X-ray (SEM-EDX) images of a single FCC particle slice show that the black spot contains $\mathrm{Fe}, \mathrm{Si}$ and $\mathrm{O}$. The scale bar is valid for all images. (b) Electron paramagnetic resonance (EPR) spectra of fraction F5 at different temperatures show a single broad band with a g-factor of 2.25 and (c) UV-vis diffuse reflectance spectroscopy (DRS) data of several clusters indicated on the images with 1 to 5 . The absorption band around $27400 \mathrm{~cm}^{-1}$ can be ascribed to a ${ }^{6} \mathrm{~A}$ to ${ }^{4} \mathrm{E}$ transition, the absorption bands from $20830-18900 \mathrm{~cm}^{-1}$ originate from $2\left({ }^{6} \mathrm{~A}_{1}\right)$ to $2\left({ }^{4} \mathrm{~T}_{1}\right)$ transitions and give rise to the color of the iron oxide.

a)

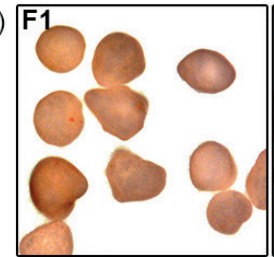

b)

\begin{tabular}{|r|c|c|}
\hline & $\begin{array}{c}\text { area \% with } \\
\text { aggregates }\end{array}$ & \# particles \\
\hline F1 & 0.029 & 200 \\
\hline F2 & 0.030 & 193 \\
\hline F3 & 0.14 & 94 \\
\hline F4 & 0.37 & 149 \\
\hline F5 & 1.4 & 73 \\
\hline
\end{tabular}

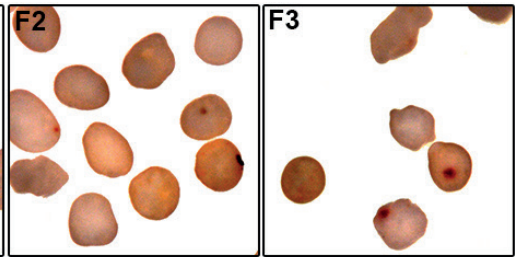

c) Particle distribution

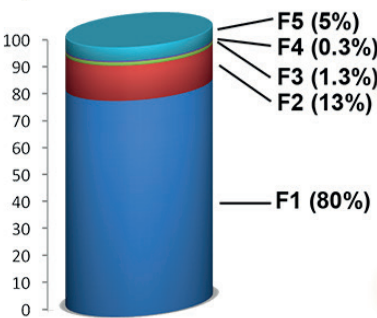

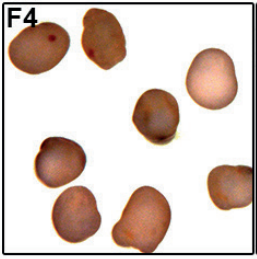

d)
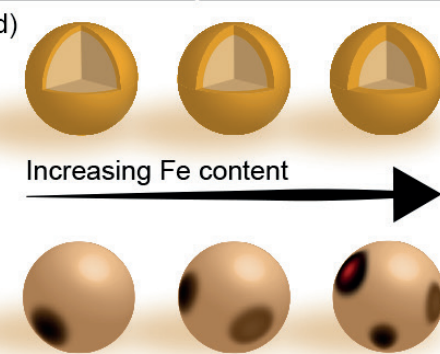

Figure 2.6: Optical images (a) of the sorted ECAT fractions, showing an increasing number of dark spots on their surface. The full particles are used and imaged with a large numerical aperture, visualizing their outer surface. The table (b) shows the ratio of aggregate area to particle area for each fraction. In (c) the particle distribution is shown, as calculated from following the trajectories of all sorted particles with a Grasshopper high speed camera. In (d) an image shows the formation of a Fe shell or Fe clusters on the surface of ECAT particles.

In Figure 2.6, the percentage of the particles' visible outer surface (projected to 2-D) that is covered by these aggregates is listed per fraction. To do this, the images were binarized with a manually set threshold to count the area of the aggregates and particles 
per 2-D image. The percentage of the 2-D visible particle surface that was found covered by black or red aggregates was then calculated, showing an increasing covered area from fractions F2 to F5. As previously mentioned, it is known that Fe coming from the crude oil feedstock is deposited mainly in/on the outer rim of the FCC particle, as illustrated in Figure 2.6d. ${ }^{[10,25]}$ The FCC catalyst particle distribution over the five fractions (Figure 2.6c) indeed shows that $80 \%$ of the ECAT particles, which are those in fraction F1, follow this pathway for the deposition of Fe. However, the catalyst particles in fractions F2 to F5, which add up to $20 \%$ of the whole ECAT sample, contain an increasing amount of Fe aggregates on their surface, as illustrated in Figure 2.6d.

When magnetic material is present in bigger Fe oxide clusters, the magnetic moment at lower fields is higher. This effect could cause a larger deviation in the Fe content per fraction. For that, a further study on previously shown hysteresis loops by performing a Langevin fit was performed. The conventional model for superparamagnetism ${ }^{[26]}$ was used to estimate the volume of the magnetic clusters from the magnetic hysteresis loops. We assume the particles to be composed of a number of magnetic clusters with identical volume and moment. From the Langevin fit, one can estimate the moment of the clusters. Assuming a saturation magnetization for magnetite $(\mathrm{Ms}=480 \mathrm{kA} / \mathrm{m})$, we can estimate the cluster volume per fraction.

To calculate the range of average cluster sizes inside the ECAT particles and their anisotropy constant, the zero-field cooled (ZFC) and fieldcooled (FC) technique were used. With this technique, the magnetic moment of a sample is measured when heating with (FC) and without (ZFC) previously applied magnetic field. From the temperature at which the ZFC moment coincides with the FC, which is the blocking temperature $\mathrm{TB}$, and taking $\mu\left(\mathrm{K}_{\mathrm{B}} \mathrm{T}\right)^{-1}$ from the Langevin fitting, the average cluster size and anisotropy constant can be extracted. The small step at 5 $\mathrm{T}$, visible in all loops, is attributed to the PPMS and not to the sample. This was demonstrated by recognizing the same step in a measurement with no sample. Figure 2.7 presents the ZFC and FC results of fractions F1, F3 and F5, showing very different behaviors at temperatures below
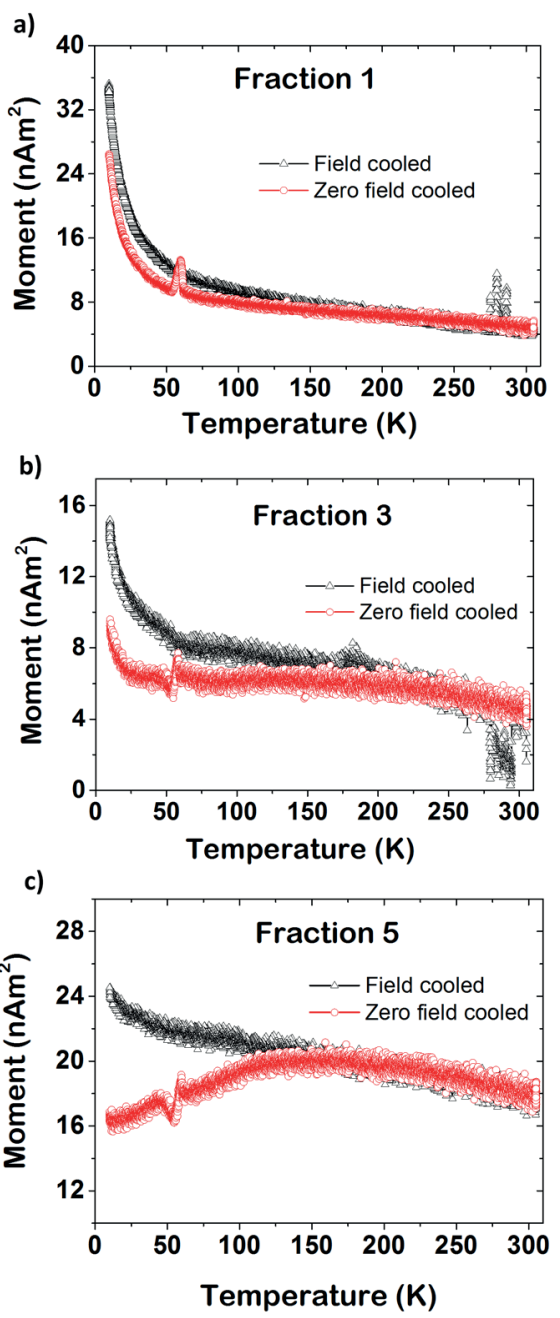

Figure 2.7: $\mathrm{ZFC}$ and FC results of sorted ECAT particles (a) F1 $=500$ particles, (b) F3 $=100$ particles (c) $F 5=500$ particles. 
$50 \mathrm{~K}$. However, when comparing $\mathrm{TB}$, no significant differences between the fractions are observed. Taking the TB of fraction $\mathrm{F} 5$ as $150 \mathrm{~K}$, we find the ratio of $\mathrm{K}(\mathrm{Ms})^{-1}$ to be 0.23 . This value is 10 times higher than that of typical magnetic iron oxides of the form of magnetite and maghemite, 0.04 and 0.02, respectively. Assuming that in fraction F5 the Fe content is $\sim 3 \mathrm{wt} \%$, similar to the HML ECAT particles from Meirer et al. ${ }^{[27]}$, the anisotropy constant and the saturation magnetization can be calculated ${ }^{[28]}$ resulting in $17 \mathrm{~kJ} / \mathrm{m}^{3}$ and $73 \mathrm{kA}(\mathrm{m})^{-1}$; much lower than that of maghemite and magnetite. We can also calculate the average radius of the clusters to be $9 \mathrm{~nm}$. This confirms that during the cracking process most of the Fe is deposited as clusters with sizes of a few nm, big orange and black Fe aggregates included. Considering that, in fractions F1 and F5, when Fe content increases with a factor of 2 the magnetic moment increases with a factor of 13 and that FC measurements show very different behaviors at low temperature, it is suggested that Fe deposited during FCC is in another ${ }^{[10,12]}$ and more magnetic form. The complexity and number of Fe (oxide) types and knowing that the magnetic moment of Fractions F2-5 could be caused by a combination of these different forms only allows for
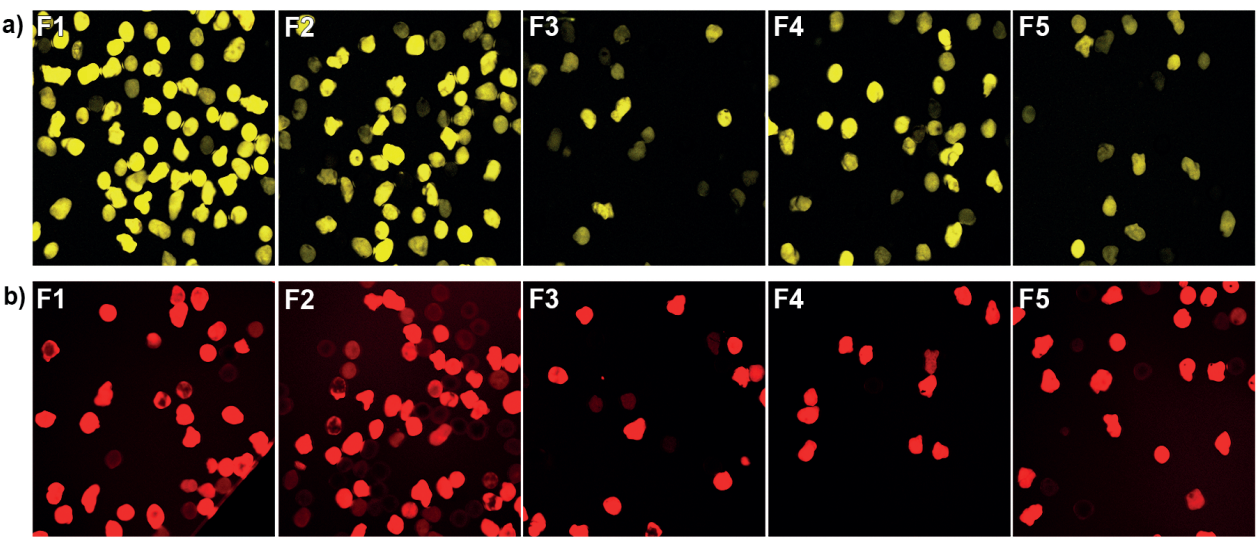

- 100 micrometer

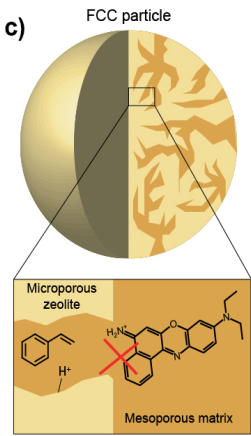

d)

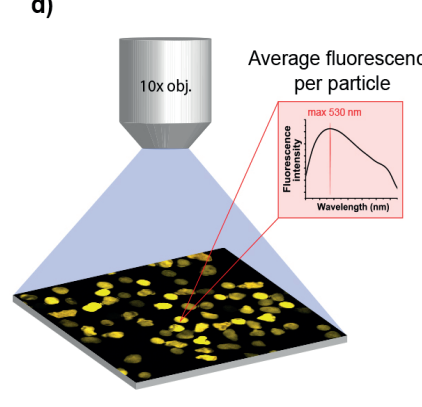

e)

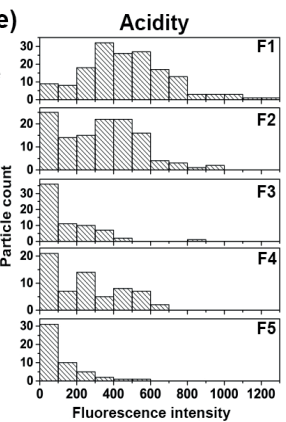

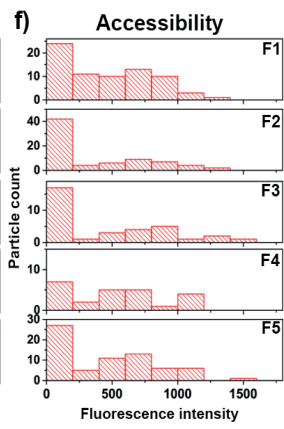

Figure 2.8: The fluorescence microscopy images that were used for determining the average fluorescence intensity per particle for 4-methoxystyrene (a) and Nile Blue A (b) staining. The schematic image of Nile blue A and styrene staining (c) shows how the zeolite shape selectivity prevents Nile blue A from entering the zeolites' microporous structure. Fluorescence micro-spectroscopy images (d) were collected and analyzed per particle. Histograms show the average fluorescence intensity per ECAT particle of (e) 4-methoxystyrene oligomers as a measure for acidity and (f) Nile blue as a measure for pore accessibility. 
a speculative explanation about its final form.

To verify whether these Fe clusters are related to a decrease in the amount of available Brønsted acid sites (after this referred to as acidity) due to pore blocking, staining experiments were performed using 4-methoxystyrene and Nile blue A. Previous work demonstrated that the Bronsted acid-catalyzed oligomerization of 4-methoxystyrene visualizes zeolite domains by fluorescence micro-spectroscopy. ${ }^{[16-18]}$ This fluorescence was used as a measure for acidity by averaging the fluorescence intensity per particle over \pm 100 ECAT catalyst particles for all five fractions.

These average intensities were compared with Nile blue A staining results. In contrast to 4-methoxystyrene, which reacts within the zeolite, Nile blue A is a molecule that can, due to its size, only enter the meso- and macropores of FCC particles (Figure 2.8c). ${ }^{[16]}$ Therefore, it can be used as a measure of meso- and macro-pore accessibility. It is expected that with increasing metal accumulation, both acidity and pore accessibility drop. ${ }^{[27]}$ However, when we look at the accessibility and acidity of the magnetically sorted fractions we see different behavior. In order to assess this in detail we have performed staining experiments with 4-methoxystyrene and Nile Blue A in combination with fluorescence micro-spectroscopy. Figure 2.8a and b show example images of the fluorescence intensity for 4-methoxystyrene and Nile Blue A per fraction. The brightness and contrast are adjusted to visualize the non-fluorescent microscopy images. A single particle analysis was performed by placing multiple ECAT particles in the field of view of the fluorescence microscope (Figure 2.8d). For each particle, an average fluorescence spectrum was measured, and the emission maximum was used to create intensity histograms for both staining agents per fraction. For obtaining the histograms with acidity and accessibility measurements per fraction (Figure 2.8e and $\mathrm{f}$ ), images with spectral information of multiple ECAT particles were analyzed by determining an average fluorescence per particle using ROI imaging in the Confocal Nikon Al analysis software. In general, the spread within all the fractions is large, meaning that every fraction still contains very active and deactivated particles. In Figure 2.8e and 2.8f, it can be seen that in fraction $\mathrm{F} 1$, containing $80 \%$ of all ECAT particles, most particles show a high fluorescence signal, which can be related to high acidity. Although a very clear decrease in acidity is detected, the particles in the fractions with higher Fe content are not all deactivated. Furthermore, there is no significant change in the accessibility histograms. For example, in Fraction F4, there are still ECAT particles with high acidity and high accessibility; in fact, the particles with surface Fe aggregates show very high fluorescence intensity. This means that when an aggregate of Fe is present, the rest of the particle can still be accessible for catalysis. This indicates that the presence of Fe aggregates does not automatically imply the presence of Fe deposited as a shell. The decrease in acidity can be caused by other deactivating processes like, for example, dealumination. ${ }^{[19]}$

\subsection{Conclusions}

A newly developed 3D-printed microfluidic chip has been used to magnetophoretically sort a set of industrially used Fluid Catalytic Cracking (FCC) catalyst particles into 5 
distinct fractions, which have been characterized in more detail with various analytical methods. The trends in magnetic moment of the sorted FCC particles were found to correlate with Fe content; it is suggested that the Fe deposited during FCC is in another ${ }^{[10,12]}$ and more magnetic form than the common $\mathrm{Fe}_{\mathrm{X}} \mathrm{O}_{\mathrm{Y}}$. Also, low temperature magnetization measurements show different behaviors at low temperatures, but similar blocking temperature between the fractions. These magnetic observations strongly point into the direction that variation in magnetic moment is caused by an increased number, different form but similar size of iron-containing nanoclusters. In addition, on average, when the Fe content increases, the average acidity of the catalyst generally decreases. However, the spread within acidity of each separated catalyst fraction is still large: not all highly magnetic catalyst particles show a high degree of deactivation. We believe that the developed separation technology could also be used to sort other magnetic catalysts for analysis purposes. An example being Fe-based Fischer-Tropsch synthesis catalysts. The approach can also serve as a valuable tool to decrease the heterogeneity of to be analyzed samples from an industrial FCC reactor unit or from other reactors, containing e.g. Fischer-Tropsch catalysts.

\subsection{Acknowledgements}

We thank Hans de Boer (University of Twente, UT), Mark Smithers (UT), Mark Huijben (UT), Hans Keizer (UT), Kristel Jagtenberg (Utrecht University, UU) and Yoni Nederstigt (UU) for their respective contributions to this work.

\subsection{References and Note}

[1] A. K. Au, W. Huynh, L. F. Horowitz, A. Folch, Angew. Chem. Int. Ed. 2016, 55, 3862-3881.

[2] Y. Gao, Y. C. Jian, L. F. Zhang, J. P. Huang, J. Phys. Chem. C 2007, 111, 10785-10791.

[3] N. Pamme, Lab Chip 2006, 6, 24-38.

[4] N. Pamme, A. Manz, Anal. Chem. 2004, 76, 7250-7256.

[5] F. L. and U. L. Martin A. M. Gijs, Chem. Rev. 2010, 110, 1518-1563.

[6] N. Pamme, C. Wilhelm, Lab Chip 2006, 6, 974-980.

[7] N. M. Karabacak, P. S. Spuhler, F. Fachin, E. J. Lim, V. Pai, E. Ozkumur, J. M. Martel, N. Kojic, K. Smith, P. Chen, J. Yang, H. Hwang, B. Morgan, J. Trautwein, T. A. Barber, S. L. Stott, D. Shyamala, A. Haber, M. Toner, Nat. Protoc. 2014, 9, 694-710.

[8] E. P. Furlani, J. Appl. Phys. 2006, 99, 1-35.

[9] D. Mance, J. van der Zwan, M. E. Z. Velthoen, F. Meirer, B. M. Weckhuysen, M. Baldus, E. T. C. Vogt, Chem. Commun. 2017, 53, 3933-3936.

[10] A. M. Wise, J. N. Weker, S. Kalirai, M. Farmand, D. A. Shapiro, F. Meirer, B. M. Weckhuysen, ACS Catal. 2016, 6, 2178-2181.

[11] J. Ihli, R. R. Jacob, M. Holler, M. Guizar-Sicairos, A. Diaz, J. C. Da Silva, D. Ferreira Sanchez, F. Krumeich, D. Grolimund, M. Taddei, W. C. Cheng, Y. Shu, A. Menzel, J. A. Van Bokhoven, Nat. Commun. 2017, 8, 809.

[12] J. Ihli, D. Ferreira Sanchez, J. Rosh, V. Cuartero, O. Mathon, F. Krumeich, C. Borca, T. Huthwelker, W.-C. Cheng, Y.-Y. Shu, S. Pascarelli, D. Grolimund, A. Menzel, J. A. van Bokhoven, Angew. Chem. Int. Ed. 2017, 56, 14031-14035.

[13] T. L. Goolsby, H. F. Moore, Sep. Sci. Technol. 1997, 32, 655-668.

[14] L. R. Aramburo, S. Wirick, P. S. Miedema, I. L. C. Buurmans, F. M. F. De Groot, B. M. Weckhuysen, Phys. Chem. Chem. Phys. 2012, 14, 6967-6973.

[15] Z. Ristanovi , M. M. Kerssens, A. V. Kubarev, F. C. Hendriks, P. Dedecker, J. Hofkens, M. B. J. Roeffaers, B. M. Weckhuysen, Angew. Chem. Int. Ed. 2015, 54, 1836-1840.

[16] I. L. C. Buurmans, J. Ruiz-Martínez, W. V Knowles, D. Van Der Beek, J. A. Bergwerff, E. T. C. Vogt, B. M. Weckhuysen, Nat. Chem. 2011, 3, 862-867. 
[17] M. M. Kerssens, A. Wilbers, J. Kramer, P. De Peinder, G. Mesu, B. J. Nelissen, E. T. C. Vogt, B. M. Weckhuysen, Faraday Discuss. 2016, 188, 69-79.

[18] I. L. C. Buurmans, J. Ruiz-Martínez, S. L. Van Leeuwen, D. Van Der Beek, J. A. Bergwerff, W. V Knowles, E. T. C. Vogt, B. M. Weckhuysen, Chem. Eur. J. 2012, 18, 1094-1101.

[19] S. Kalirai, P. P. Paalanen, J. Wang, F. Meirer, B. M. Weckhuysen, Angew. Chem. Int. Ed. 2016, 55, 1113411138.

[20] E. L. Kugler, D. P. Leta, J. Catal. 1988, 395, 387-395.

[21] A. S. Escobar, M. M. Pereira, R. D. M. Pimenta, L. Y. Lau, H. S. Cerqueira, Appl. Catal. A Gen. 2005, 286, 196-201.

[22] E. T. C. Vogt, B. M. Weckhuysen, Chem. Soc. Rev. 2015, 44, 7342-7370.

[23] W. R. Hagen, Dalt. Trans. 2006, 4415-4434.

[24] R. M. Cornell, U. Schwertmann, in Iron Oxides Struct. Prop. React. Occur. Uses, Wiley-VCH Weinheim, 2004, pp. 139-183.

[25] F. Meirer, D. T. Morris, S. Kalirai, Y. Liu, J. C. Andrews, B. M. Weckhuysen, J. Am. Chem. Soc. 2015, 137, $102-105$.

[26] C. P. Bean, J. D. Livingston, J. Appl. Phys. 1959, 30, S120-S129.

[27] F. Meirer, S. Kalirai, D. Morris, S. Soparawalla, Y. Liu, G. Mesu, J. C. Andrews, B. M. Weckhuysen, Sci. Adv. 2015, 1, el400199.

[28] The magnetic moment $\mathrm{m}\left(\mathrm{Am}^{2}\right)$ of the particle as function of the magnetic field $\mathrm{B}(\mathrm{T})$ is fitted to a Langevin curve: $m(B)=m_{0} L\left(\mu_{B} / k_{B} T\right)$, (1) where $m_{0}$ is the moment of the particle at saturation, equal to the number of magnetic clusters times their moment $\mu, \mathrm{k}_{\mathrm{B}}$ is Boltzmann's constant $\left(1.38 \times 10^{-23} \mathrm{~J} / \mathrm{K}\right)$ and $\mathrm{T}$ the measurement temperature $(\mathrm{K})$. The susceptibility of the particle, $\chi_{\mathrm{L}}=\partial \mathrm{m} / \partial \mathrm{B}$, is approximately equal to: $\chi_{\mathrm{L}}=\mathrm{m}_{0} \mu_{\mathrm{B}}\left(3 \mathrm{k}_{\mathrm{B}} \mathrm{T}\right)^{-1}(2)$ for temperatures above the blocking temperature. From the Langevin fit, one can estimate the moment of the clusters. Assuming a saturation magnetization for magnetite $(\mathrm{Ms}=480 \mathrm{kA} / \mathrm{m})$, we can estimate the cluster volume $V_{\text {cluster }}=\mu / M_{s}$ (3) We define the temperature at which the cluster loses its magnetization within the time of measurement $\left(\tau_{m}\right)$, the blocking temperature $T_{B}: \tau_{m}=\tau_{0} \exp \left(\left(K_{\text {cluster }}\right) /\left(k_{B} T_{B}\right.\right.$ )), (4) where $\tau_{0}$ is the attemt time for magnetisation reversal in the cluster and $K$ is the magnetic anisotropy of the material (at most $20 \mathrm{~kJ} / \mathrm{m}^{3}$ for magnetite). For VSM measurements one usually assumes $\ln \left(\tau_{\mathrm{m}} / \tau_{0}\right)$ to be approximately 25 , so we have a second estimate for the volume of the cluster of $V_{\text {cluster }}=\left(25 \mathrm{k}_{\mathrm{B}} \mathrm{T}_{\mathrm{B}}\right) / \mathrm{K}(5) \mathrm{By}$ combining (3) and (5) we obtain $\mathrm{K}=\left(25 \mathrm{k}_{\mathrm{B}} \mathrm{T}_{\mathrm{B}} \mathrm{M}_{\mathrm{S}}\right) / \mu$ (6) 


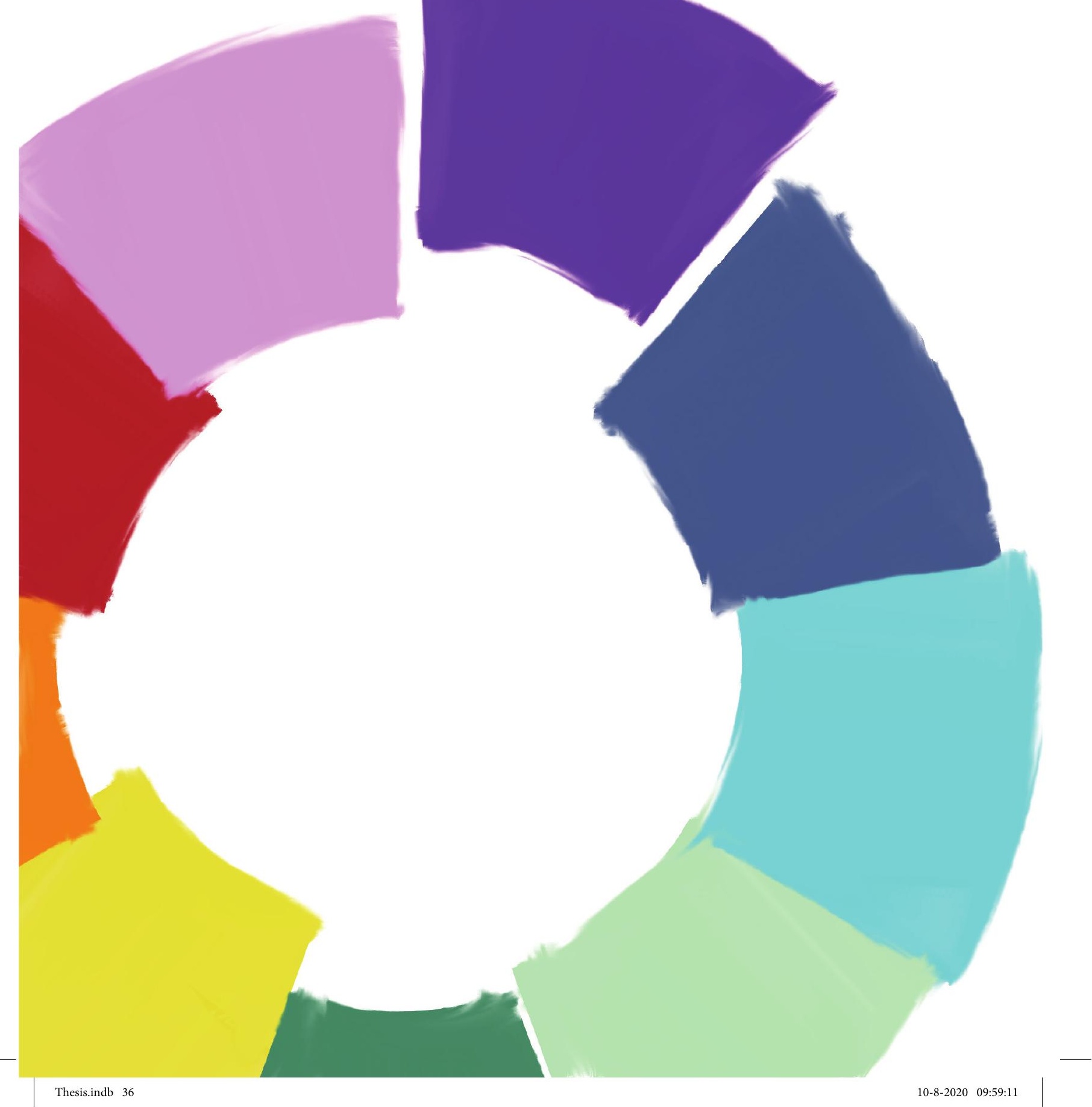




\section{Chapter 3}

\section{Single Particle Essays to Determine Heterogeneities within Fluid Catalytic Cracking Catalysts}

Fluid Catalytic Cracking (FCC) is an important process in the oil refinery industry to produce gasoline and propylene. Due to harsh reaction conditions, FCC catalysts are subject to deactivation through e.g. metal accumulation and zeolite framework collapse. In this Chapter, we perform a screening of the influence of metal poisons on the acidity and accessibility of an industrial FCC catalyst material using laboratory-based single particle characterization i.e., $\mu-X R F$ and fluorescence microscopy in combination with probe molecules. These methods have been performed on density-separated FCC catalyst fractions, allowing to determine interparticle heterogeneities in the catalyst under study. It was found that with increasing catalyst density and metal content, the acidity and accessibility of the catalyst particles decrease, while their distribution narrows with catalyst age. For example, particles containing high Ni levels possess very low acidity and are hardly accessible by a Nile Blue A dye. Single catalyst particle mapping identifies minority species like the presence of a phosphated zeolite ZSM-5-containing FCC additive for selective propylene formation, catalyst particles without any zeolite phase, and catalyst particles which act as a trap for $\mathrm{SO}_{\mathrm{x}}$.

This work is based on the following manuscript: A.-E. Nieuwelink*, M.E.Z. Velthoen*, Y.C.M. Nederstigt, K.L. Jagtenberg, F. Meirer, B.M. Weckhuysen, Single Particle Essays to Determine Heterogeneities within Fluid Catalytic Cracking Catalysts, Chem. Eur. J., 2020, 26, 8546-8554.

*These authors contributed equally. 


\subsection{Introduction}

In the past decade, our research group has performed extensive work on the characterization of Fluid Catalytic Cracking (FCC) particles. Buurmans et al. used several chemical staining techniques, inspired by life science methods, to visualize the acidity of industrially retrieved (further denoted as equilibrium or ECAT) catalysts and laboratorydeactivated FCC catalysts at the single particle level. ${ }^{[1,2]}$ These staining techniques have been combined with for example transmission electron microscopy (TEM) to correlate the acidity with structural features in one FCC particle. ${ }^{[3]}$ In later work by Ristanovic et al., extensive confocal fluorescence microscopy (CFM) work revealed more details on the activity of single zeolite domains by performing single molecule activity determination.

${ }^{[4]}$ Kerssens et al. later on performed staining experiments on bigger samples of a few milligrams, to visualize the acidity of both fresh, deactivated FCC and industrially retrieved ECAT particles. ${ }^{[5]}$ For the accumulation of metals, Ruiz-Martínez et al. revealed a core-shell deactivation due the accumulation of metals and coke deposits using noninvasive 2D micro X-ray fluorescence ( $\mu$-XRF). With 3D X-ray microscopy (XRM) experiments, Meirer et al. have studied the distribution of deactivating metals, such as $\mathrm{Fe}$ and $\mathrm{Ni}$, in more detail. They have performed a density separation to classify three categories (i.e., low, medium and high metal loaded FCC particles) and found that Fe forms a shell of reduced porosity in the outer 2-3 $\mu$ m layer of the FCC particle, while Ni is distributed more throughout the whole catalyst particle. ${ }^{[6,7]}$ However, a disadvantage of single catalyst particle characterization methods is the difficulty to obtain statistically relevant data. ${ }^{[8]}$ For example, due to the use of expensive synchrotron-based techniques, ${ }^{[9]}$ the amount of particles that can be analyzed is rather limited. Although the previously mentioned studies provided very useful physicochemical insights on catalyst deactivation, they unfortunately only focus on limited amounts of particles.

In Chapter 2, we have developed a method for the individual assessment of multiple FCC particles via microfluidic sorting, based on the catalyst particles' magnetic moment. The sorted samples obtained were, however, still too small to perform bulk characterization techniques, but it was already possible to analyze a significant number of $\sim 700$ particles on the single particle level. ${ }^{[10]}$ For this analysis, after sorting the FCC particles, all collected fractions were analyzed with for example X-ray fluorescence microscopy (XRF) and fluorescence microscopy using staining molecules such as 4-methoxystyrene and Nile blue A, to characterize the fractions' metal content, acidity and accessibility.

In this Chapter, we continue this line of single particle diagnostics research on FCC catalyst particles by presenting a study about the influence of different deactivation processes on their activity and accessibility. In particular, we report a characterization approach, using laboratory-based techniques, that bridges the gap between single particle and bulk analyses. This is schematically illustrated in Scheme 3.1. We use the same set of characterization techniques as reported in Chapter 2 and try to further correlate the single particle mapping data of the different ECAT fractions sorted by a classical density separation. Such a correlation to reveal interparticle heterogeneities has previously 
been reported for the analysis of polymerization catalysts. ${ }^{[11]}$ We use these correlations to establish relevant relationships as well as to identify special FCC catalyst particles. For this purpose, a calcined ECAT material was sorted into six fractions, using density gradient separation with different diiodomethane/acetone mixtures. ${ }^{[12]}$ The increasing density per fraction is attributed to the accumulation of metals like $\mathrm{Fe}, \mathrm{Ni}$ and $\mathrm{V}$ and, thus, to the catalytic age of a particle. It is generally excepted that the activity of an FCC particle decreases with increasing metal loading. ${ }^{[13]}$ By sorting this ECAT material on density, the interparticle heterogeneity within each age fraction is reduced to facilitate a more precise characterization. Bulk characterization was established with traditional methods, such as inductively coupled plasma-optical emission spectroscopy (ICP-OES), $\mathrm{NH}_{3}$-temperature programmed desorption (TPD) and $\mathrm{N}_{2}$-physisorption. In this work, the term 'bulk technique' is used to address techniques that analyze a relatively large sample of ECAT particles and give an ensemble average as outcome. Each density separated

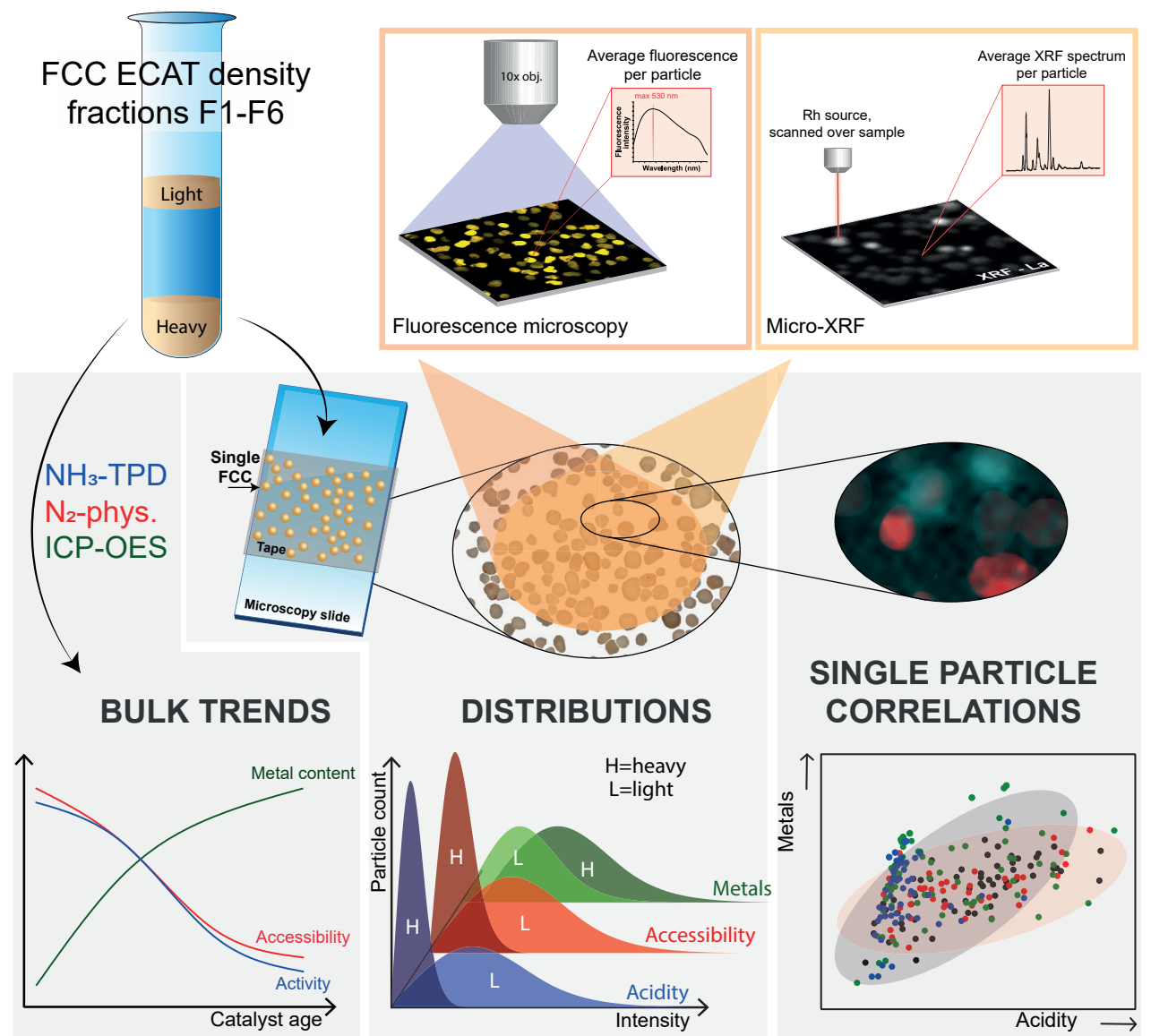

Scheme 3.1: Schematic of the approach taken in this Chapter. A Fluid Catalytic Cracking (FCC) equilibrium catalyst (ECAT) was density separated into six fractions and analyzed for its acidity, accessibility and metal content. Bulk characterization techniques revealed trends as a function of density. Micro-spectroscopic techniques were employed for large scale single particle mapping. This screening allowed for determining interparticle distributions within each fraction as well as establishing detailed single particle correlations. 
ECAT fraction was, subsequently, characterized with multiple micro-spectroscopic techniques to visualize the interparticle heterogeneity within each fraction. Specifically, fluorescence micro-spectroscopy and micro ( $\mu$-)XRF measurements were performed on a set of the same particles to obtain correlated information on many single FCC ECAT particles. As a result, valuable correlations between catalyst acidity, pore accessibility and metals accumulation could be made.

From this comprehensive approach, conclusions on three different levels could be drawn, as indicated in Scheme 3.1. First, bulk trends regarding the acidity, accessibility and metal accumulation as a function of catalyst age were established. Second, within each density separated fraction, large scale single particle mapping revealed the level of heterogeneity within these catalyst fractions. Finally, this particle screening resulted in detailed correlations regarding the catalyst properties and highlighted the presence of minority species that cannot be detected in neither bulk nor single particle analysis. The key of this presented methodology relies on single particle analysis with statistical relevance.

\subsection{Experimental Section}

\subsubsection{Sample Preparation}

A regenerated FCC ECAT obtained from an industrial FCC unit was dried at $120{ }^{\circ} \mathrm{C}$ for $1 \mathrm{~h}$ (ramp: $1{ }^{\circ} \mathrm{C} / \mathrm{min}$ ), followed by calcination at $600{ }^{\circ} \mathrm{C}$ for $5 \mathrm{~h}\left(5^{\circ} \mathrm{C} / \mathrm{min}\right)$ to remove residual coke species. Subsequently, density gradient separation as described by Dyrkacz et al. ${ }^{[14]}$ was used to sort the FCC ECAT particles in six fractions with increasing density. For this purpose, diiodomethane (DIIM, Sigma-Aldrich, purity 99\%)/acetone (GPR RECTAPUR, VWR Chemicals, purity $>99.5 \%$ ) mixtures with different ratios were prepared as listed in Table 1. For the first sorting step, $2.5 \mathrm{~g}$ FCC ECAT particles was added to a $20 \mathrm{~cm}$ tube $(\Phi=1 \mathrm{~cm})$ and dispersed in $15 \mathrm{~mL}$ of solution 1 . After $2 \mathrm{~h}$, the particles were equilibrated, and two layers were created: a heavy fraction at the bottom of the tube and a floating fraction on top of the DIIM/acetone solution. The upper layer was removed, washed with acetone and dried in air. This fraction is herein referred to as fraction F1. The lower layer was re-dispersed in $15 \mathrm{~mL}$ of DIIM/acetone solution 2 to split off a second, slightly denser, fraction that was treated in a similar manner as fraction $\mathrm{F} 1$ and is herein referred to as fraction F2. This process was repeated with solutions 3 to 5. After the last step, also the bottom layer was washed and dried. All six fractions were

Table 3.1: Solutions used for density gradient separation of calcined Fluid Catalytic Cracking (FCC) equilibrium catalyst (ECAT) particles.

\begin{tabular}{l||l|l} 
& DIIM:acetone $(\% \mathrm{vol})$ & Calculated density $\left(\mathrm{cm}^{3} / \mathrm{g}\right)$ \\
\hline Solution 1 & $1.5: 1$ & 2.31 \\
\hline Solution 2 & $2: 1$ & 2.47 \\
\hline Solution 3 & $2.5: 1$ & 2.60 \\
\hline Solution 4 & $3: 1$ & 2.64 \\
\hline Solution 5 & $5: 1$ & 2.90
\end{tabular}


calcined following the same procedure as described above to remove residual solvent. In this way the FCC ECAT was separated into six fractions with increasing density, herein denoted as fractions F1-6.

\subsubsection{Bulk Characterization Methods}

Temperature programmed desorption (TPD) of ammonia $\left(\mathrm{NH}_{3}\right)$ measurements were performed using a Micromeritics ASAP2920 equipped with a TCD detector. Typically, $0.1 \mathrm{~g}$ of sample was dried in-situ under He flow at $550^{\circ} \mathrm{C}$ for $30 \mathrm{~min}$ (ramp $10{ }^{\circ} \mathrm{C} / \mathrm{min}$ ). The sample was subsequently cooled down to $100^{\circ} \mathrm{C}$. At this temperature, $\mathrm{NH}_{3}$ pulses of $25.17 \mathrm{~cm}^{3} / \mathrm{min}$ were applied until saturation of all acid sites. The sample was then heated to $550{ }^{\circ} \mathrm{C}$ (ramp $10{ }^{\circ} \mathrm{C} / \mathrm{min}$ ) to induce desorption of $\mathrm{NH}_{3}$. Fourier Transform Infrared (FT-IR) spectra were recorded in transmission mode on a Perkin-Elmer 2000 instrument employed with a DTGS detector using 32 scans per spectrum and a resolution of $4 \mathrm{~cm}-$ 1. Typically, $0.015 \mathrm{~g}$ of sample were pressed into self-supported wafers $(\Phi=12 \mathrm{~mm})$ and placed in a cell that allows switching between vacuum and $\mathrm{CO}$ gas. Samples were dried in-situ at $550{ }^{\circ} \mathrm{C}$ for $30 \mathrm{~min}$ ( $\mathrm{ramp} 10^{\circ} \mathrm{C} / \mathrm{min}$ ) under vacuum. $\mathrm{CO}(10 \%$ in $\mathrm{He})$ was dosed at low temperatures $\left(-188^{\circ} \mathrm{C}\right.$ ) and at low pressures (between 1.0 and $1.5 \cdot 10^{-3} \mathrm{mbar}$ ) with spectra being recorded after each pulse. $\mathrm{NH}_{3}$-TPD provides an indication of the overall acidic properties and an accurate quantification of the total amount of acid sites, while CO FT-IR spectroscopy provides more insight into the nature and strength of the different acid sites present in the studied samples. The average accessibility of FCC catalyst particles as a function of age was determined with $\mathrm{N}_{2}$-physisorption. $\mathrm{N}_{2}$-physisorption was performed at $-196{ }^{\circ} \mathrm{C}$ using a Micromeritics TriStar apparatus. Samples were dried using $\mathrm{N}_{2}$. The mesopore volumes (2-300 nm range) and Barrett-Joyner-Halenda (BJH) pore size distributions were determined using the adsorption branch of the isotherm with Aerosil 380 as a reference. Micropore volume was determined with the tPlot using the linear part of the adsorption branch between 0.275 and $0.3 \mathrm{~nm}$ (statistical thickness) with Aerosil 380 as a reference. With a quantitative Inductively Coupled Plasma Optical Emission Spectroscopy (ICP-OES) measurement, nature and abundance of all metals present in the FCC ECAT fractions can be determined. ICP-OES measurements were performed using the SPECTRO ARCOS ICP-OES spectrometer. Typically, 100-150 mg of sample was dissolved in $\mathrm{HF}$ and $\mathrm{HNO}_{3}$ to remove the Si/Al framework of the catalyst. The samples were measured in an emission range of 125-770 nm.

\subsubsection{Single Particle Characterization}

We have studied the accessibility and acidity of single FCC particles with staining experiments using fluorescence microscopy. It is known from previous research in our group, that staining methods using probe molecules like thiophenes, styrenes or furfuryl alcohol can be used to study the acidity of zeolites. ${ }^{[2,8,15-17]}$ These staining methods are based on a Brønsted acid-catalyzed oligomerization reaction of the probe molecules. Upon oligomerization, conjugated reaction products are formed that can be detected with UVVis, optical or fluorescence spectroscopy. ${ }^{[5,18,19]}$ 4-Methoxystyrene was selected here due to its relatively high reaction rate constant: the methoxy group stabilizes the carbocation 
formed via the formation of resonance structures. ${ }^{[20]}$ This makes 4-methoxystyrene also active towards oligomerization on weaker Brønsted acid sites. In contrast, 4-fluorostyrene can only oligomerize on the very acidic Brønsted acid sites of e.g. zeolite ZSM-5 and is therefore, more selective, but less relevant for this study. Additionally, a non-reactive bulky molecule, like Nile blue A, can be used to probe the accessibility of the FCC particles' pore structure. Nile blue A cannot enter the zeolite pore structure, but it can enter the meso- and macroporous structure of the matrix. Therefore, the fluorescence of the FCC particles caused by Nile Blue A can be taken as a measure for the catalyst's accessibility for vacuum gas oil (VGO) molecules. ${ }^{[5,8,18,21,22]}$

4-Methoxystyrene and Nile Blue A show fluorescence in the same wavelength regions. Therefore, the experiments with Nile Blue A to probe the accessibility of the density separated FCC ECAT fractions have been performed on a new sample from the same fraction. The sample was immersed in a $0.1 \mathrm{mM}$ Nile blue A solution in ethanol. After an equilibration time, the fluorescence intensity per particle was measured. We expect that the uptake of Nile blue A by an FCC particle is dependent on the number of accessible pores. Therefore, we use the fluorescence intensity of an FCC particle after Nile blue A staining as a measure for accessibility of the particles' matrix.

Each exact same sample was also analysed employing $\mu$-XRF mapping to measure the metal content per particle. $\mu$-XRF spectroscopy is a spectrochemical tool for determining in a spatially resolved way the composition of (typically inorganic) materials. Upon excitation with a focused X-ray beam, characteristic X-rays are emitted by the sample. The detected XRF spectra can be fitted to identify the elements present in a material, with a spatial resolution that is determined by the size of the X-ray beam. ${ }^{[23]}$ The $\mu$-XRF method in this study was only used to measure relative intensities. The reference material required to perform a correct calibration, for example a fresh FCC catalyst from the same reactor unit, was not available. However, these results give valuable information about the trends within the different particles from the density separated ECAT fractions. For these measurements, each fraction was placed on tape to create a surface covered with particles. Two duplicates were prepared and placed in the chamber of a $\mu$-XRF Orbis PC SDD with a Rh tube as X-ray source. An XRF map was collected with $30 \mu \mathrm{m}$ resolution and a $15 \mu \mathrm{m}$ step size. Next, using a selfmade MATLAB script, the maps were segmented to combine the spectra per particle into one average spectrum for every particle. This allowed for the analysis of 100 individual FCC particles per fraction. Consecutively, one of the XRF samples was stained with Nile Blue A and the other with 4-methoxystyrene. The Nile Blue A staining was performed by immersing the particles in a $0.1 \mathrm{mM}$ solution of Nile Blue A and the sample was equilibrated for $1 \mathrm{~h}$. 4-Methoxystyrene was added via diffusion by placing a $10 \mu \mathrm{L}$ droplet next to the sample. In a closed environment, the sample was heated to $100{ }^{\circ} \mathrm{C}$ for $15 \mathrm{~min}$. Both samples were illuminated with a Nikon upright Al confocal fluorescence microscope equipped with a $488 \mathrm{~nm}$ excitation solidstate laser source. Images were collected using a 10x objective to fit $\sim 80 \mathrm{FCC}$ particles in one image. 
The fluorescence microscopy images and $\mu$-XRF maps were complemented with an optical image of the same region. These images were collected using a Zeiss Plan Neo Fluar upright microscope. With a second in-house designed MATLAB script, the fluorescence and optical images and the XRF maps were digitally resampled to the same pixel size and then registered to overlay all information of each individual particle. This registration step resulted in a multi-dimensional image where each pixel contained information from all used imaging methods. This allowed generating correlation plots of all present metals, the fluorescence intensity, and the observed color of the particles.

\section{F1}
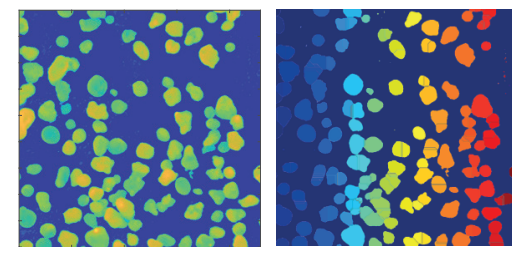

F2

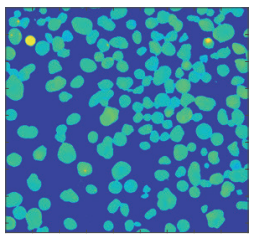

F3

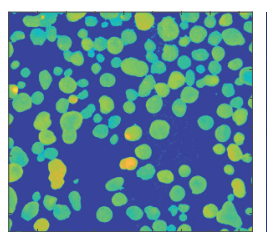

F4

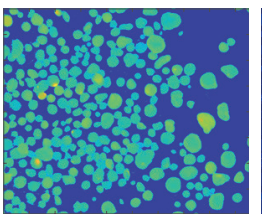

F5
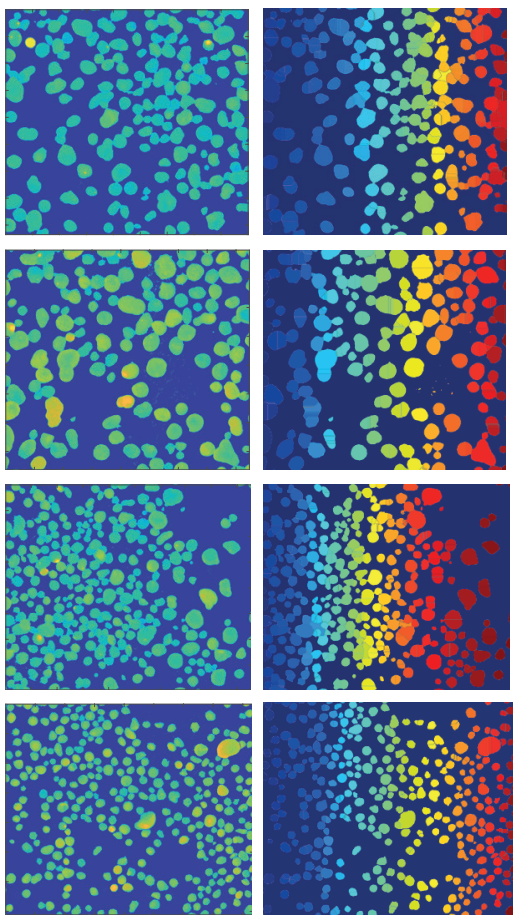

F6
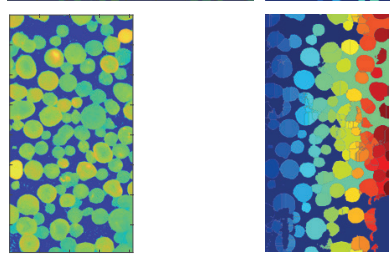

Figure 3.1: Binary images (left) and corresponding watershed (right) of every density separated fraction.
Single particle segmentation was achieved by a watershed segmentation of the $\mu-X R F$ maps. First, corresponding optical images were converted into binary images using adaptive thresholding applying Bradley's method, i.e. by calculating a threshold for each pixel using the local mean intensity around the neighborhood of the pixel. ${ }^{[24]}$ Then, the Euclidean distance transform of the binary image was computed, inverted and the watershed transform was applied. The watershed transform treats an image as a surface where dark pixels represent low and light pixels represent high elevations. In that way the watershed transforms finds so called 'watershed ridge lines' that indicate a separation of regions ('drainage basins') by elevations. ${ }^{[25]}$ When applied to the inverted distance map, the watershed can, in this way, efficiently separate two overlapping circular objects. Sometimes the watershed shows several separate particles instead of one full large particle, but mostly, the watershed is a good representation of the particles as observed in the images of Figure 3.1. After the watershed transformation, the identified regions were labeled individually, as displayed in the right part of the figure where each region is indicated by a different color (from dark blue to dark red from left to right in the map). Next, the intensities in each region were determined from the $\mu-\mathrm{XRF}$ and fluorescence images using the segmented image as a mask. This provided an average XRF/fluorescence intensity for each individual particle identified in the images and enabled the correlation analysis. The described 
approach was implemented using an in-house developed MATLAB code. For fraction F6, one of the segmentations includes a part of the background. This area was excluded from further correlation analyses.

\subsection{Results and Discussion}

A regenerated ECAT from a commercial FCC unit was sorted in six fractions using a density gradient separation protocol, which was based on a sink-float method in different acetone/diiodomethane (DIIM) mixtures. Figure 3.2a and Table 3.2 demonstrate that most FCC catalyst particles end up in the fractions with the lowest density. More specifically, more than $60 \%$ of the ECAT particles has a skeletal density below $2.47 \mathrm{~g} / \mathrm{cm}^{3}$. In comparison, depending on the amount of zeolite material present and the source of binder material and clay, skeletal densities between 2.4 and $2.8 \mathrm{~g} / \mathrm{cm}^{3}$ have been reported for fresh FCC catalyst particles. ${ }^{[13,26,27]}$

Table 3.2: Weight percentage (of $2.5 \mathrm{~g}$ ) and average density of FCC ECAT fractions obtained after density gradient separation in DIIM/Acetone mixtures. Density of mixture is calculated based on ratio of DIIM and acetone; it is assumed that these liquids are fully miscible. Percentages are based on total collected amount.

\begin{tabular}{l||l|l} 
& Amount $(\%)$ & Density $\left(\mathrm{g} / \mathrm{cm}^{3}\right)$ \\
\hline Fraction 1 & 39.4 & $<2.31$ \\
\hline Fraction 2 & 37.7 & $2.31-2.47$ \\
\hline Fraction 3 & 19.5 & $2.47-2.60$ \\
\hline Fraction 4 & 0.66 & $2.60-2.64$ \\
\hline Fraction 5 & 0.46 & $2.64-2.90$ \\
\hline Fraction 6 & 2.22 & $>2.90$
\end{tabular}

\subsubsection{Bulk Catalyst Characterization Trends}

Bulk characterization techniques, in this work referred to as techniques that were applied to a large sample of ECAT particles and including temperature programmed desorption (TPD) of $\mathrm{NH}_{3}, \mathrm{~N}_{2}$-physisorption, and inductively coupled plasma-optical emission spectrometry (ICP-OES), revealed quantitative bulk trends as function of density in acidity, accessibility and metal accumulation respectively. Together, these bulk analyses provide useful insights into the level of catalyst deactivation as a function of catalytic age. In correspondence with previous studies, the metal content of FCC particles is correlated with density, while acidity and accessibility are negatively correlated with density. Since we are investigating an industrially used and regenerated ECAT, all particles already have been used in at least one cracking cycle and could, theoretically, already be considerably deactivated. Therefore, we expect a relatively low overall acidity and accessibility in the particles.

Figure $3.2 \mathrm{~b}$ indicates increasing levels of $\mathrm{Ca}, \mathrm{Fe}$, and $\mathrm{V}$ with increasing density, suggesting longer residence times in the FCC reactor. Also, the overall level of $\mathrm{Ni}$ is relatively low in this specific FCC ECAT. These ICP-OES results demonstrate that density gradient separation of an FCC ECAT indeed leads to fractions with increasing 


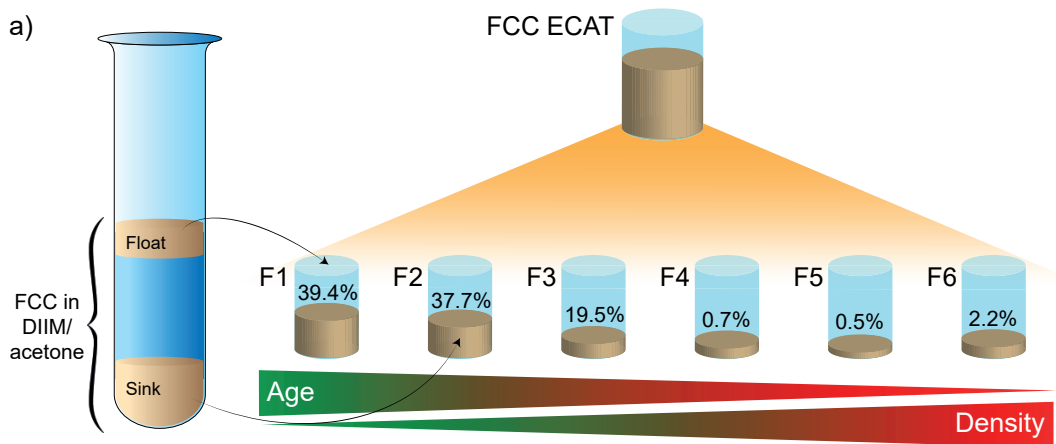

b) Metal accumulation
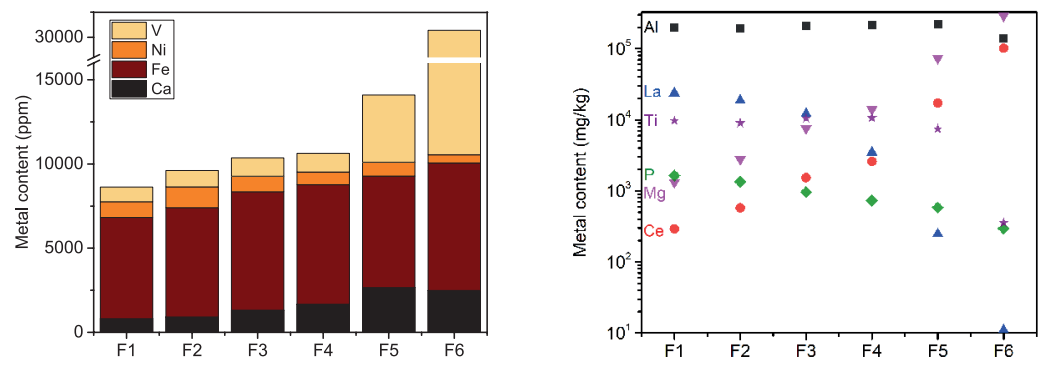

c) Acidity
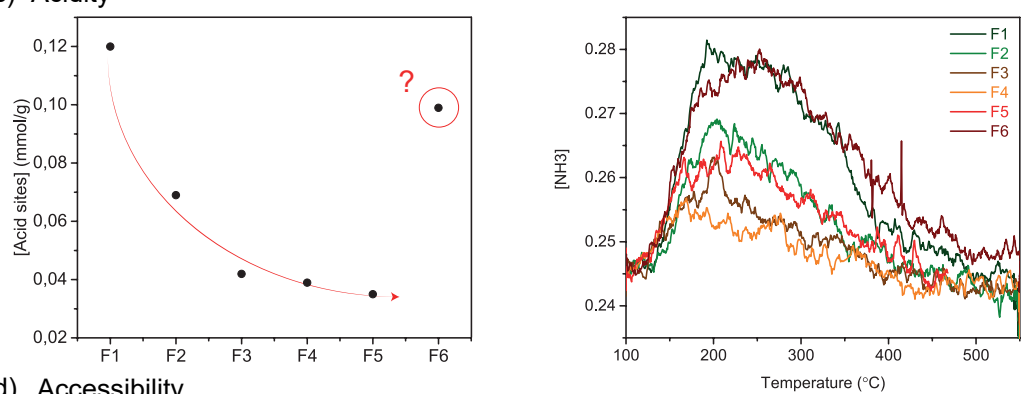

d) Accessibility
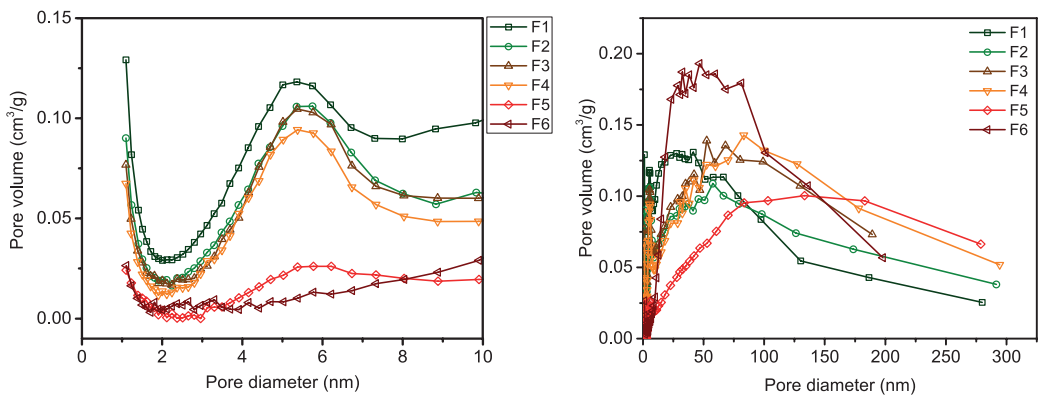

Figure 3.2: a) An FCC ECAT was density separated using a sink-float method in different acetone/ diiodomethane (DIIM) mixtures. The percentage of particles collected per fraction is indicated. Bulk characterization methods showed trends in metal accumulation, acidity and accessibility. ICP-OES data (b, NB: logarithmic scale) of important deactivating metals (left) and markers for FCC components (right) are shown. Quality control samples indicating the accuracy of the measurements give $\mathrm{Fe}=98 \%, \mathrm{Ni}=102 \%, \mathrm{~V}=101 \%$, $\mathrm{Ca}=98 \%$. Quantified NH3-TPD results (c), $\mathrm{N}_{2}$-physisorption results (d) their corresponding graphs show a decreasing acidity and amount of mesopores. The micropore volume per fraction is $\mathrm{F} 1=0.058, \mathrm{~F} 2=0.030$, $\mathrm{F} 3=0.027, \mathrm{~F} 4=0.019, \mathrm{~F} 5=0.003$ and $\mathrm{F} 6=0$. The outlier $\mathrm{F} 6$ will be discussed in the main text. 
loading of deactivating metals and, therefore, with increasing age. A sub-division is made in metals that can be used as markers and metals coming from the VGO that accumulate in the particles during catalysis. It can be seen that $\mathrm{Mg}$ and Ce increase gradually while La drops significantly going from fraction F1 to F6. These trends are addressed in more detail later.

$\mathrm{N}_{2}$-physisorption was employed to determine the accessibility properties of each fraction under study. Figure 3.2d presents the mesopore size distribution and the micropore volume. Fractions F1-4 express similar pore size distributions with considerable micropore volumes and a significant amount of small mesopores $(6 \mathrm{~nm})$, which are both inherent to the zeolite domains. Fraction F5, on the other hand, lacks these features and demonstrates a broad distribution of larger mesopores, ascribed to the matrix of FCC particles, confirming the absence of zeolite domains. Fraction F6 has a completely different pore size distribution, containing mainly pores of around $50 \mathrm{~nm}$. Comparing fractions F1-4, it can be observed that the micropore volume decreases significantly with a factor 2 going from fraction 1 to 2 and then slowly decreases further with increasing density to fraction 4. This crystallinity loss is ascribed to the dealumination and consequent structural collapse of the zeolite framework by steam during the regeneration of the FCC particles. The zeolite mesopores are more resistant against ageing.

The activity of an FCC particle in the cracking reaction depends on the presence of acid sites accessible to oil molecules, both in the matrix and in the zeolite domains. The matrix contains Lewis acid sites, capable of pre-cracking the large oil molecules and the zeolite domains possess Brønsted acid sites to crack the molecules into the desired products. Particles with a higher degree of dealumination and metal accumulation are, therefore, expected to possess a lower amount of acid sites and, by extension, to be less active. To support this hypothesis, the acidic properties of the six fractions under study were determined and taken as a measure for activity. $\mathrm{NH}_{3}-\mathrm{TPD}$ characterization resulted in a quantified amount of acid sites per gram, as indicated in Figure 3.2c. There is a clear decrease in the amount of acid sites per gram with increasing density. Going from fraction F1 to F2, the amount of acid sites decreases significantly, similar to what was observed for the accessibility with $\mathrm{N}_{2}$-physisorption. Then, the amount of acid sites gradually decreases further during the ageing of FCC particles (fractions F1-4). The acidity of fraction F5 is low, due to the absence of zeolite domains. To obtain more insights into the nature of the different acid sites present in the different fractions, FT-IR spectroscopy with CO as a probe molecule for acid sites was employed.

The FT-IR spectra as depicted in Figure 3.3, show a decreasing amount of Brønsted acid sites and isolated silanol groups with increasing sample density. Furthermore, it can be observed that strong Lewis acid sites have a low abundance in fractions F1 and F2 but are negligible in higher density fractions. The previously observed decrease in accessibility is also expressed by the amount of physisorbed CO confined in pores: the intensity of this band strongly decreases in fraction F2 in comparison with fraction F1.

Three striking observations can be made from these bulk trends. First, as seen in 

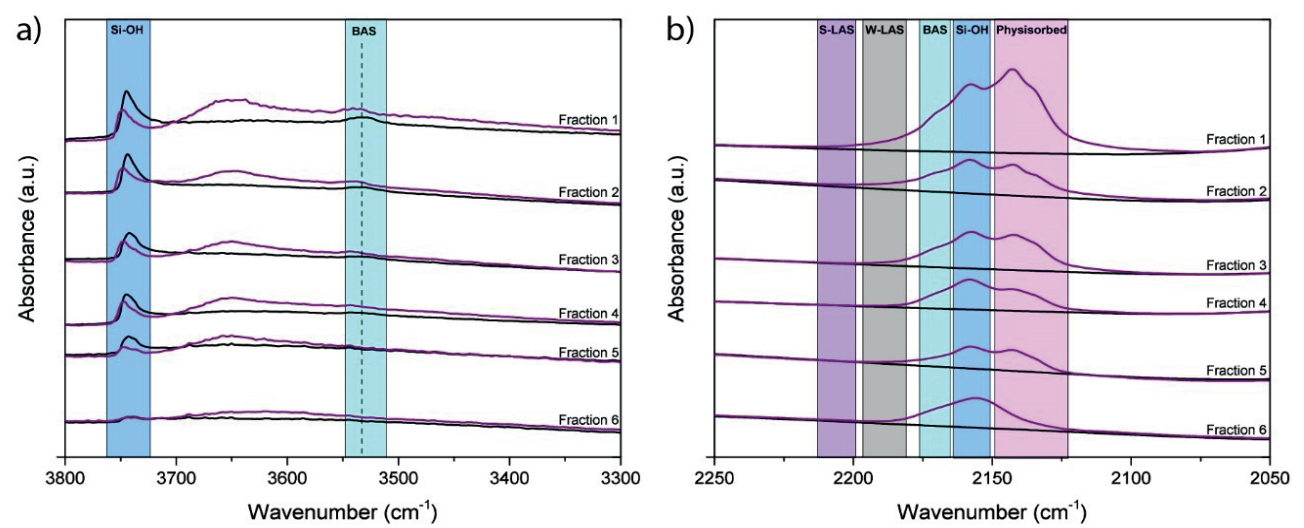

Figure 3.3: FT-IR spectra collected before (black) and after $\mathrm{CO}$ adsorption (purple) for all fractions in the $\mathrm{OH}$ vibrational region (a) and CO vibrational region (b) Spectra are corrected for the weight of the selfsupporting wafer and plotted with an offset for clarity. CO adsorbed to Bronsted acid sites (BAS), strong Lewis acid sites (S-LAS), weak Lewis acid sites (W-LAS) and Si-OH groups are indicated as well as physisorbed CO.

Figure 3.2c, the decrease in acidity appears most significant going from fraction F1 to F2 and from F2 to F3, while fractions F3 to F5 have similar acidities. CO FT-IR spectroscopy indeed confirms that most Brønsted acid sites are removed going from fraction F1 to F2 and indicate that strong Lewis acid sites even only exist in fraction F1. Also, mesopores of around $6 \mathrm{~nm}$ and the micropore volume decrease significantly from fraction F1 to F4. This is a clear indication of the destruction of zeolite domains, the only microporous material in an FCC particle, during the aging of the particle. Furthermore, the mesopores of 20$30 \mathrm{~nm}$ as observed in fraction F1, disappear completely upon ageing. This is ascribed to the accumulation of metals in the matrix of the particles, blocking the accessibility for oil molecules.

Second, both fractions F5 and F6 appear to lack a presence of zeolite domains, as both fractions demonstrate a low level of La compared to fractions F1-4. The La level can be used as a marker for zeolite US-Y domains, since this rare earth metal is used as stabilizing agent during the synthesis of this zeolite. ${ }^{[28]}$ Therefore, fraction F5 and F6 contain nonFCC particles without zeolite domains. Considering only fractions comprising actual FCC particles (fraction F1 to F4), their La levels are fairly similar for fractions F1 to F3, which is an indication for a comparable amount of zeolite domains in these fractions, and slowly decreases to fraction F4. From literature, it is not expected that La will leave the particles upon zeolite framework collapse. ${ }^{[29]}$ The absence of zeolite domains in fractions F5 and F6 is further rationalized by the deviating pore size distribution as indicated in Figure 3.2d. These two observations indicate that employing density gradient separation allows for filtering out most non-FCC particles from the ECAT batch.

Third, the composition of fraction F6, the heaviest fraction, does not follow the trends set by the first five fractions. The $\mathrm{Mg}$ and $\mathrm{V}$ levels are particularly high in this most dense FCC fraction, while as shown in Figure 3.2b the level of Ti, a marker for the clay component of a typical FCC particles' matrix ${ }^{[30]}$ is very low. This suggests that fraction F6 mainly contains Mg-based traps for vanadium or $\mathrm{SO}_{x}{ }^{[31-33]}$ which we were able to 
separate from the FCC catalyst particles using density separation. These particles possess a low porosity, but have a relatively high Lewis acidity, most probably due to the presence of acidic vanadium oxide.

\subsubsection{Particle Distributions within a Density-Separated Fluid Catalytic Cracking} Catalyst

The bulk characterization techniques, as discussed in Figure 3.2, provide overall trends and insights in the investigated ECAT material. Single particle mapping, however, provides a more in-depth analysis of the interparticle heterogeneity of the investigated FCC samples. These results give distributions of the acidity, accessibility and metal accumulation in every fraction. For every fraction, a minimum of 90 particles was analyzed. The exact numbers are given in Table 3.3.

Table 3.3: The number of particles that was analyzed per density sorted fraction. Depending on the number of particles in field of view of the X-ray map or fluorescence microscope.

\begin{tabular}{l||l|l|l} 
& $\begin{array}{l}\text { \# of particles } \\
\text { Acidity }\end{array}$ & $\begin{array}{l}\text { \# of particles } \\
\text { Metals }\end{array}$ & $\begin{array}{l}\text { \# of particles } \\
\text { Accessibility }\end{array}$ \\
\hline \hline Fraction 1 & 74 & 229 & 29 \\
\hline Fraction 2 & 168 & 347 & 76 \\
\hline Fraction 3 & 218 & 267 & 54 \\
\hline Fraction 4 & 135 & 463 & 49 \\
\hline Fraction 5 & 71 & 378 & 54 \\
\hline Fraction 6 & 101 & 322 & 36
\end{tabular}

Figure 3.4a shows histograms of the fluorescence intensity per particle after the Brфnsted acid catalyzed oligomerization of 4-methoxystyrene. This fluorescence intensity is a measure for the number of available acid sites. With this staining method, using a styrene derivative as probe molecule, the amount of available acid sites can be visualized, as was shown before by Buurmans et al..$^{[1,2,8]}$ In line with our previously shown bulk results in Figure 3.2c, the acidity decreases with density. For an assessment of the particles' accessibility, staining experiments were performed using Nile blue A, a large conjugated fluorescent molecule that can enter the macro- and mesoporous pore structure of an FCC particle. ${ }^{[8]}$ Similar to the acidity measurements in Figure 3.4a, the fluorescence intensity from Nile Blue A reflects the particle accessibility. The distributions in Figure $3.4 \mathrm{~b}$ indicate that also the average accessibility decreases with increasing density.

Strikingly, the trends in the acidity distributions show an almost bimodal behavior. Within the fractions F1-F3, the distributions of acidity demonstrate a large spread and thus significant interparticle heterogeneities. Fractions F4-F6, however, show more narrow distributions with low fluorescent intensities. We ascribe the heterogeneity in the lighter fractions to a convolution of a natural density spread and ageing. Due to the spray drying process, we expect a density gradient already in the fresh catalyst. Particles that are, therefore, denser from the start, can end up in a fraction together with aged particles. Fraction F4 only shows non-fluorescent particles, suggesting the absence of acid sites and 
a)

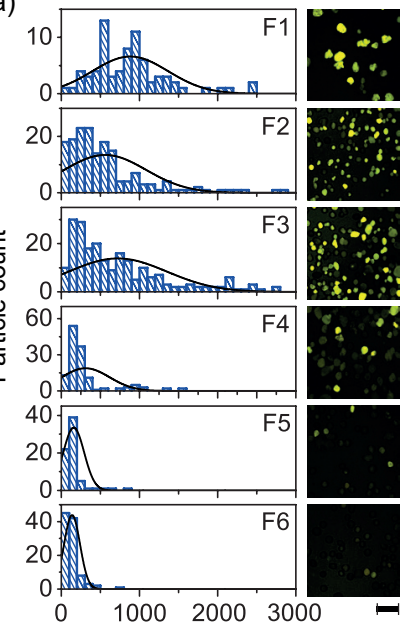

Styrene fluorescence intensity (average counts) b)
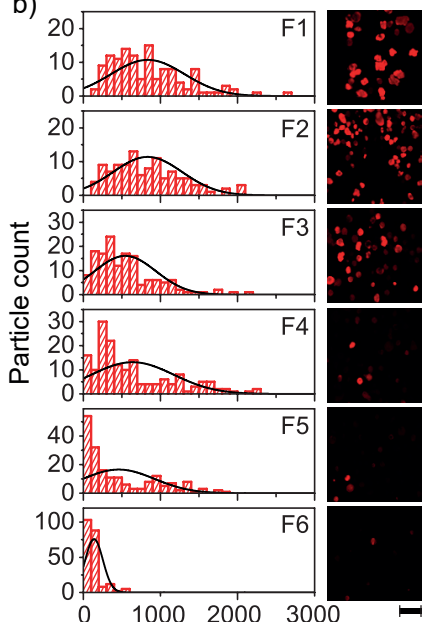

Nile Blue fluorescence intensity (average counts)

Figure 3.4: Single particle mapping revealing interparticle distributions within each density separated ECAT fraction. For each particle in field of view, the fluorescence of styrene oligomerization reaction products indicates the level of acidity (a) and the fluorescence of Nile Blue A demonstrates the accessibility (b). Scale bars equal $200 \mu \mathrm{m}$.

the blockage of pores. The decrease in porosity is less pronounced than the decrease in acidity. The large spread in the accessibility of particles in Fraction F4 suggests that the deactivation of Fraction F4 is due to a combination of pore blocking by Fe, as reported by Meirer et al. and the absence of acid sites. ${ }^{[13,34]}$

A different explanation for the non-fluorescent particles in all fractions, relies on the presence of other type of particles, such as additives or spray-dried particles without zeolites. The absence of Brønsted acid sites can be a result of synthesis or due to severe dealumination. Bulk analysis showed that such particles are typically found in fractions F5 and F6. Regardless of the origin, these so-called non-FCC particles are not as porous as the active FCC particles and do not contain Bronsted acid sites to perform the oligomerization of 4-methoxystyrene. Due to a low metal loading, however, they can

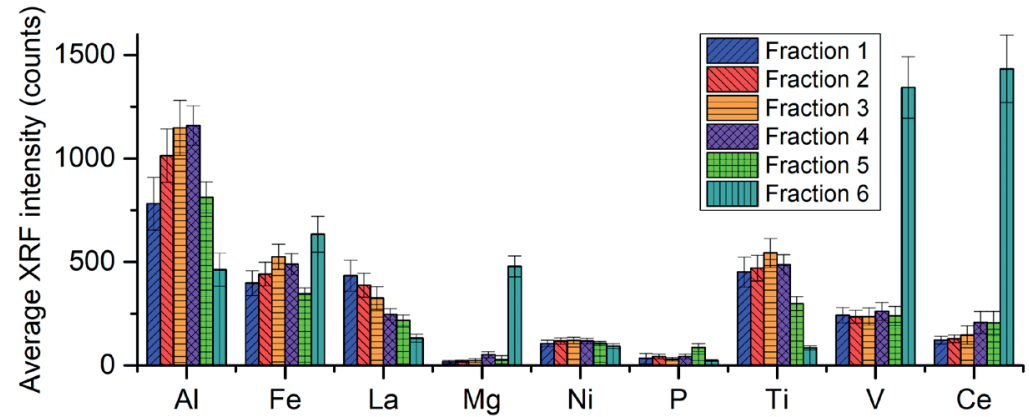

Figure 3.5: Average $\mu-\mathrm{XRF}$ values of all elements measured of the sample used for correlations. For the $\mu \mathrm{XRF}$ experiments, the average values are determined from the complete $\mu$ XRF maps. This includes the background values for every element. All elements that are not shown here, could not be measured with XRF due to the experimental setup. The error bars correspond to the standard deviation that rises from averaging the XRF values of multiple particles in the $\mu \mathrm{XRF}$ map. 
also end up in the lightest fractions. Although they show low fluorescence intensities regarding acidity and accessibility, they are properly sorted into the correct age fraction.

Finally, $\mu$-XRF experiments provided the XRF intensity of different metals per particle. The average $\mu$-XRF values per element and fraction (Figure 3.5) follow the trends as previously observed with bulk analysis (ICP-OES). This means that the single particle mapping method provides an accurate representation of the bulk material, indicating that we have studied a statistically relevant number of particles. The main advantage of this $\mu$-XRF mapping over bulk methods like ICP-OES, however, is that interparticle heterogeneity is revealed.

In Figure 3.6, the distributions of $\mathrm{Fe}, \mathrm{V}$ and $\mathrm{Ni}$ as a function of density are shown. The Fe distributions appear narrower compared to styrene and Nile Blue A in Figure 3.4. Also, the differences between fractions are less pronounced and more difficult to visualize. Therefore, to show clear trends and capture outliers with either very low or high Fe contents, we need more particles as compared to the acidity and accessibility studies. It is interesting to see the broad V distribution of fraction F6, while the other fractions contain only limited amounts of $\mathrm{V}$. The distributions of $\mathrm{Ni}$ are narrow and at relatively low intensities and indicate an overall low amount of $\mathrm{Ni}$ in this ECAT. All $\mu$-XRF images per fraction are of the same region and can therefore be directly overlayed.
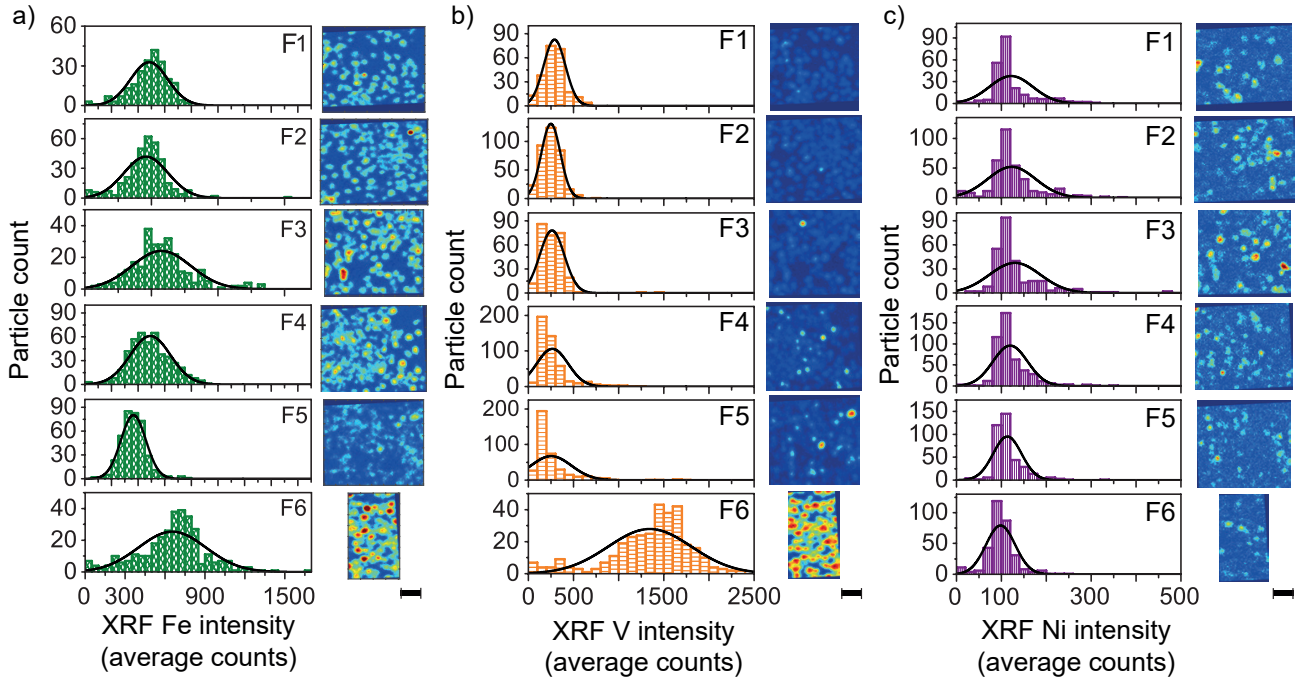

Figure 3.6: Single particle mapping revealing interparticle distributions within each density separated ECAT fraction. For each particle in field of view, the XRF intensity represents the content of Fe (a), V (b) and $\mathrm{Ni}$ (c). Each scale bar equals 200 to $\mu \mathrm{m}$.

\subsubsection{Correlations between Metal Type and Content and Catalyst Particle Acidity \& Accessibility}

By performing $\mu-X R F$ and fluorescence microscopy measurements at identical positions, single particle mapping can also provide correlations between the acidic properties and the metal content of particles. These correlations allow for detecting the 
presence of minority species, provide additional information about the FCC ECAT, or reveal new trends that were not revealed with bulk analyses.

In FCC deactivation research, the Ni content of a particle is often used as indication for the age of a catalyst: $\mathrm{Ni}$ is accumulated during the cracking process but is not present in the fresh catalyst (such as Fe in the clay component of the FCC catalyst). Furthermore, $\mathrm{Ni}$ inside an FCC particle is not very mobile. Therefore, the more Ni an ECAT particle contains, the longer it has been cycling through the reactor and the more Fe and V it contains. ${ }^{[35]}$ Consequently, it is expected that these particles show a low acidity and accessibility and thus a low fluorescence of Nile Blue A and 4-methoxystyrene.

Correlation plots show the relation between fluorescence, $\mathrm{Fe}$ and $\mathrm{Ni}$, where each measured particle is represented with one data point. These plots originate from the overlay of the corresponding $\mu$-XRF maps and the resulting single particle intensities. A detailed explanation of the data analysis can be found in Section 3.2.3. F2 The overlaying maps of acidity and Ni in Figure 3.8a demonstrate an anti-correlation between acidity and $\mathrm{Ni}$ content in each fraction. Closer inspection reveals that particles with a high Ni content always show low fluorescence, and F3 therefore low acidity. We can therefore conclude that, indeed, Ni acts as a marker for the catalytic age of an ECAT. It must be noted that a low level of acidity is indeed linked to a higher degree of deactivation and not to an absence of zeolite $F$ domains, as indicated in Figure 3.7. Due to the overall low levels of $\mathrm{Ni}$ present in this ECAT, bulk characterization techniques were not sufficiently accurate to detect this new trend. The correlation of Ni with NB staining (Figure F5 $3.8 \mathrm{~b}$ ) is less pronounced, but also here particles with a high Ni content show low accessibility. The correlation between $\mathrm{Fe}$ and acidity or accessibility is not as straightforward. We expect a relation where acidity and accessibility decrease with increasing Fe level, due to pore blocking. However, the overlay images in Figure 3.8 show particles with a high Fe content that still show 4-methoxystyrene (left) or Nile Blue A (right) fluorescence. In these particles, Fe is most likely deposited as clusters. These Fe clusters

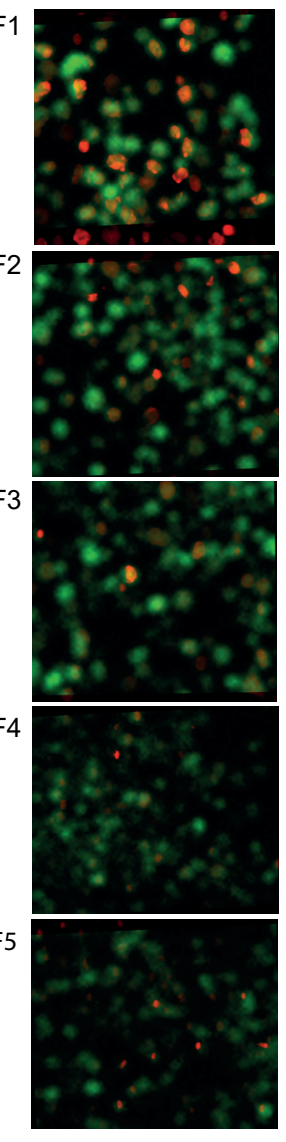

$\bigcirc$ La $\bigcirc$ Fluo

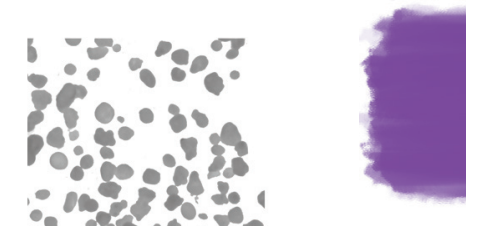


a)
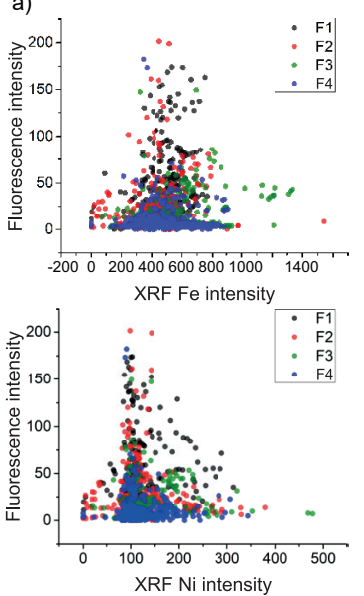

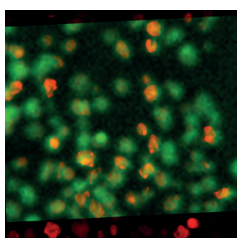

Fluo $\bigcirc \mathrm{Fe} \bigcirc \mathrm{Ni}$

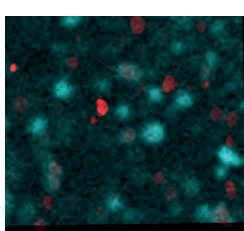

$\mapsto 200 \mu \mathrm{m}$
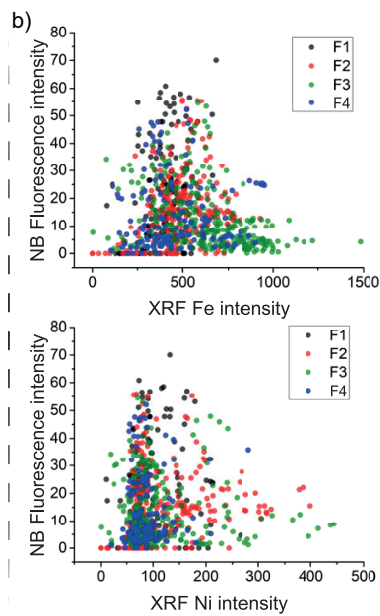

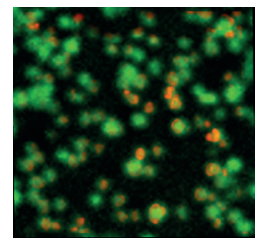

Fluo $\bigcirc \mathrm{Fe} \bigcirc \mathrm{Ni}$

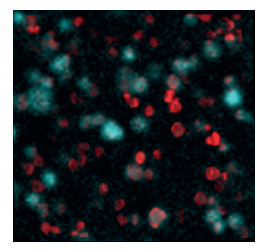

$\longmapsto 200 \mu \mathrm{m}$

Figure 3.8: The correlation plots of $\mathrm{Fe}$ (green in image) and $\mathrm{Ni}$ (blue in image) with fluorescence (intensity in average counts) of 4-methoxystyrene (a) and Nile blue (b) with corresponding example images show the relation between deactivating metals and acidity/accessibility. Pearson correlations of these plots are -0.014 , $0.0011,-0.11$ and 0.049 , indicating there is no linear correlation found in all plots.

a)
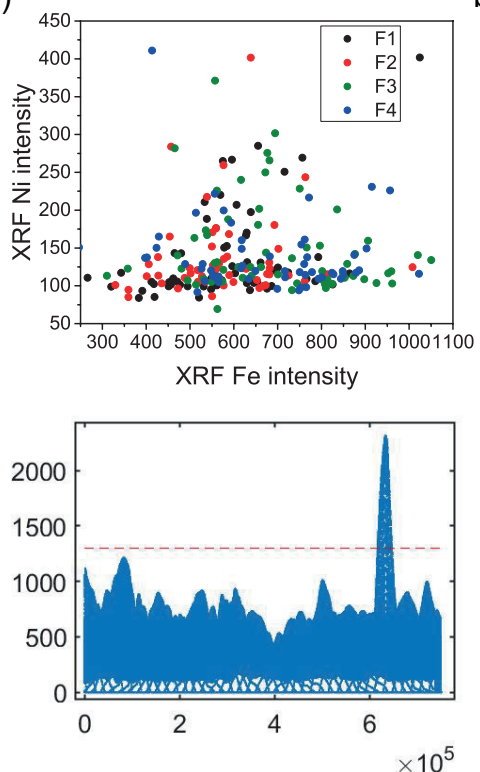

b)

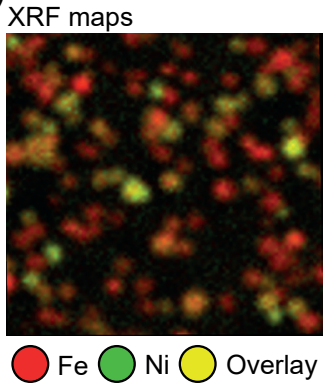

c)
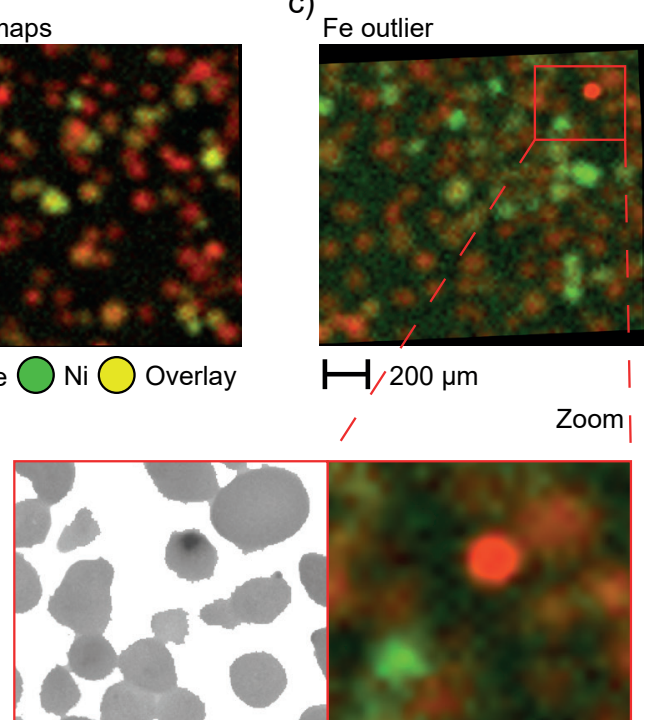

Figure 3.9: The correlation plot of the XRF maps of Ni and Fe (a, Pearson correlation: 0.26). Every data point represents an individual particle with Pearson correlation coefficients of $0.54,0.16,-0.12,-0.18$ for F1 to F4 respectively. The overlay images (b) of fraction 2 are shown as an example. The second example (c) shows a Fe outlier with a very high XRF intensity, attributed to a cluster. The threshold of the overview Fe image is chosen as such to display the overall image correct; the outlier is therefore saturated. The intensity plot for all pixels (x-axis) in the XRF Fe map of fraction F2 as displayed in the bottom right shows a red line for the cut off value of 1300 used to scale the image. 
were already observed in the previous chapter, where it was demonstrated that particles with high Fe levels are not necessarily heavily deactivated. ${ }^{[10]}$

The different correlations between $\mathrm{Fe}$ and Ni with acidity and accessibility, lead to the question how Fe and Ni relate to each other. Figure 3.9a shows the Ni-Fe correlation plots as a result of overlaying the single particle XRF maps. The correlation appears to be a fan-shaped plot, indicating that particles with high Ni concentrations generally also have elevated Fe levels. The reverse correlation, on the other hand, does not uphold, meaning that particles with a high Fe concentration do not necessarily have a high $\mathrm{Ni}$ content as well. The hotspot of Fe that is observed in the upper right corner of Figure 3.9b, is allocated to an FCC particle with a dark spot on the optical image in Figure 3.9c. This particle has, thus, a cluster with high concentrations of Fe, but overall, does not have a high skeletal density, as it was sorted in fraction F2. Interestingly, whereas bulk characterization demonstrated that the Fe and Ni contents gradually increase with increasing density, single particle mapping now indicates a significant heterogeneous interparticle distribution.

\subsubsection{Minority Species}

Our presented single particle mapping method provides the possibility to determine the composition of many single particles within each fraction. This allows for the detection of outliers and minority species. As was stated before, FCC particles can contain both zeolite ZSM-5 and US-Y as active cracking components. Like La serves as a marker for zeolite US-Y, the presence of ZSM-5 in a particle is indicated by the P content. The ICP-OES measurements in Figure 3.2b did not detect a significant amount or clear trend in the $\mathrm{P}$ content per fraction. On the single particle level, however, we observe the presence of ZSM-5 containing particles in fractions F1 and F2. Figure 3.10 shows these particles in red. Interestingly, these particles contain low levels of Fe and Ni. Second, the $\mathrm{Mg}$ based traps for $\mathrm{V} / \mathrm{SO}_{\mathrm{x}}$ are more clearly detected with $\mu$-XRF mapping.

a)

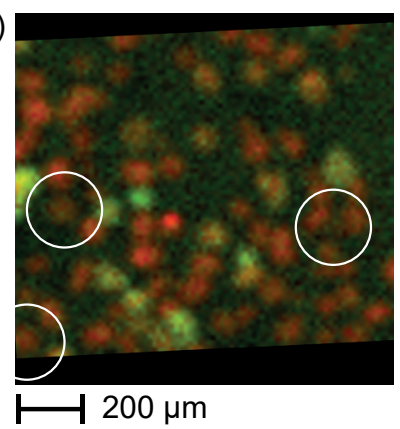

b)

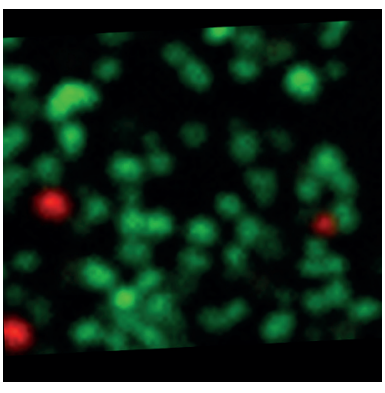

Figure 3.10: (a) An overlay of $\mathrm{Ni}$ (green) and Fe (red) in fraction F1 shows that the zeolite ZSM-5-containing catalyst particles (within white circles) generally contain below average levels of Fe and/or Ni. In (b) the ZSM5 particles are indicated red $(\mathrm{P})$ while USY particles are indicated green (La).

Detailed correlation plots in Figure 3.11 show the direct link of $\mathrm{Mg}$ with $\mathrm{V}$. The correlation plot of the $\mu-\mathrm{XRF}$ maps of $\mathrm{Mg}$ and $\mathrm{V}$ shows the linear correlation between $\mathrm{Mg}$ and V levels. The Pearson correlation coefficients are 0.08, 0.22, 0.32, 0.88 and 0.98 
a)

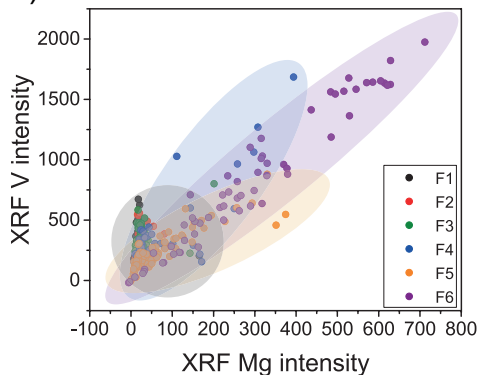

b)

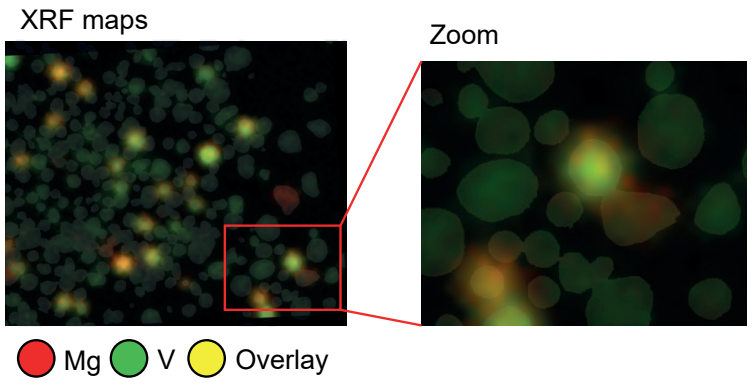

Figure 3.11: Overlay of $\mathrm{V}$ and $\mathrm{Mg}$ shows a high correlation, indicating the presence of a $\mathrm{Mg}$ based trap for $\mathrm{V}$.

for fractions F1 to F6 respectively. The overlay images of the $\mathrm{V}$ and $\mathrm{Mg} \mu$-XRF map of fraction F4 together with an optical image (15\% opacity) show an example and a zoom onto some $\mathrm{Mg}$ based $\mathrm{V}$ traps still present in this fraction.

The red indicated particles $(\mathrm{Mg})$ in Figure 3.12 demonstrate that minor amounts of these traps were collected in fractions F1 to F5, but the majority is collected in fraction F6. Particles not displaying one of the pure distinct colors red, blue, or green, do not contain (enough) zeolite domains (green, blue) or $\mathrm{Mg}$ (red) and are indicated as grey in the pie charts. It is important to note that these thresholds are chosen manually; based on the average values that were found with $\mu-\mathrm{XRF}$, similar as the method shown in Figure 3.9c. From the pie charts, once more the large intrinsic heterogeneity of an FCC ECAT is emphasized. Most striking from these results is the heterogeneity of the La values found for all particles. The large spread in zeolite content is most probably due to the used spray drying technique and again highlights the importance of a method that can perform diagnostics on many single particles: bulk methods cannot give enough information on a sample as complex as an FCC ECAT material. This characterization method can be extended to other systems with a large inter-particle heterogeneous catalysts such as polymerization catalysts or metal impregnated supports. ${ }^{[11,36]}$
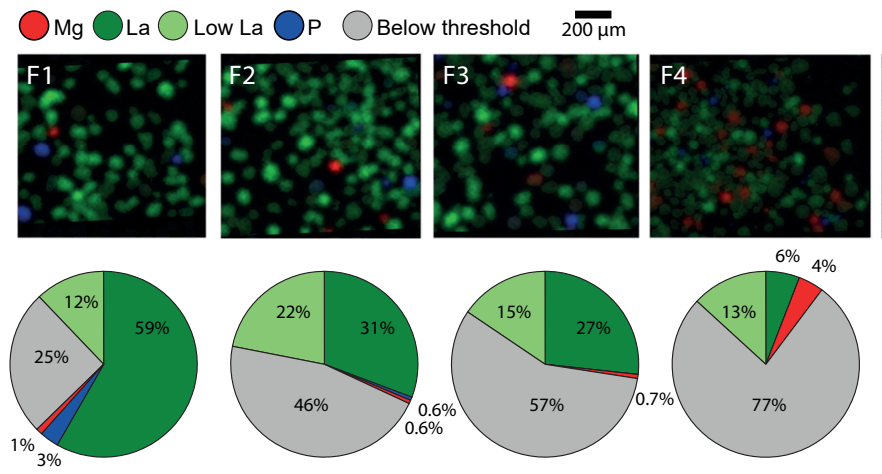

$6 \% \quad 4 \%$
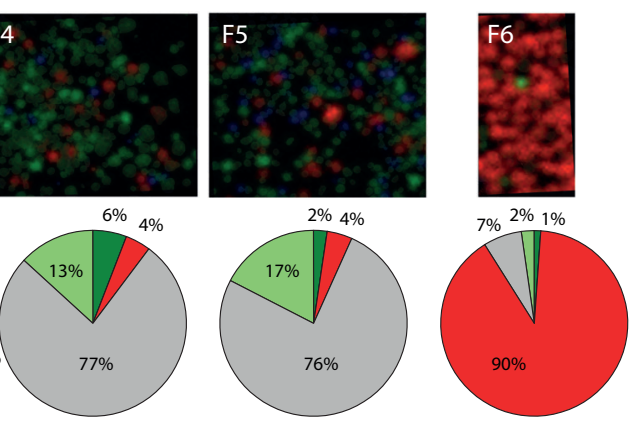

Figure 3.12: Overlay of XRF maps Mg, La and P for fraction F1-F6 and relative percentages of all three. Particles with an XRF intensity for La between 200 and 600 are indicated as light green in the pie charts. The blue color in the overlay image of fraction F5 is below the set threshold and therefore not ascribed to ZSM- 5 domains, but to other additives. The pie charts represent the percentages of the number of particles with $\mathrm{Mg}$, La or $\mathrm{P}$ as main content. 


\subsection{Conclusions}

We have shown that correlating information from multiple techniques per particle, provides valuable insights in the heterogeneity of a density separated FCC ECAT. Furthermore, the possibility to measure multiple particles adds statistical value to the data obtained. Where bulk measurements can give insight in the trends between the six density separated FCC ECAT fractions under study, the diagnostics of single particles can show trends even within each fraction.

Extensive characterization revealed clear trends in the acidity, accessibility and metal content of the density separated fractions: from fractions F1 to F4 the separation was according to catalytic age. We conclude this from the accumulation of deactivating metals, a decrease in acidity and accessibility. However, we have shown a large spread in acidity and accessibility for these fractions as a result of a convolution of natural density and ageing. Fractions F5 and F6 are mainly composed of additives without zeolites or Mg-based traps for $\mathrm{V}$ or $\mathrm{SO}_{\mathrm{x}}$.

The large intrinsic inter-particle heterogeneity of each density separated fraction was visualized by overlaying XRF maps of $\mathrm{Fe}$ and Ni with fluorescence maps. The degree of deactivation was linked to the $\mathrm{Ni}$ content on a single particle level, in a sense that particles with a high Ni content do not show high acidity. Furthermore, correlations of the $\mathrm{Mg}$, La and P levels revealed the presence of ZSM-5 containing particles in the lightest fractions, while the $\mathrm{V} / \mathrm{SO}_{\mathrm{x}}$-traps were collected with increasing amounts in the heavier fractions.

\subsection{Acknowledgements}

We thank Albemarle for financial support for the work of Marjolein Velthoen (Utrecht University, UU). Marianna Gambino (UU), Coen Mulder (UU), and Lennart Weber (UU) are thanked for helping with $\mu$-XRF data interpretation, ICP-OES measurements and N2-physisorption measurements, respectively.

\subsection{References}

[1] I. L. C. Buurmans, J. Ruiz-Martínez, S. L. Van Leeuwen, D. Van Der Beek, J. A. Bergwerff, W. V Knowles, E. T. C. Vogt, B. M. Weckhuysen, Chem. Eur. J. 2012, 18, 1094-1101.

[2] I. L. C. Buurmans, B. M. Weckhuysen, Nat. Chem. 2012, 4, 873-886.

[3] M. A. Karreman, I. L. C. Buurmans, A. V Agronskaia, J. W. Geus, H. C. Gerritsen, B. M. Weckhuysen, Chem. Eur. J. 2013, 19, 3846-3859.

[4] Z. Ristanoviccccccccccc, M. M. Kerssens, A. V. Kubarev, F. C. Hendriks, P. Dedecker, J. Hofkens, M. B. J. Roeffaers, B. M. Weckhuysen, Angew. Chem. Int. Ed. 2015, 54, 1836-1840.

[5] M. M. Kerssens, A. Wilbers, J. Kramer, P. De Peinder, G. Mesu, B. J. Nelissen, E. T. C. Vogt, B. M. Weckhuysen, Faraday Discuss. 2016, 188, 69-79.

[6] F. Meirer, D. T. Morris, S. Kalirai, Y. Liu, J. C. Andrews, B. M. Weckhuysen, J. Am. Chem. Soc. 2015, 137, 102-105.

[7] F. Meirer, S. Kalirai, J. N. Weker, Y. Liu, J. C. Andrews, B. M. Weckhuysen, Chem. Commun. 2015, 51, 8097-8100.

[8] I. L. C. Buurmans, J. Ruiz-Martínez, W. V Knowles, D. van der Beek, J. A. Bergwerff, E. T. C. Vogt, B. M. Weckhuysen, Nat. Chem. 2011, 3, 862-867.

[9] F. Meirer, B. M. Weckhuysen, Nat. Rev. Mater. 2018, 3, 324-340.

[10] M. Solsona, A.-E. Nieuwelink, F. Meirer, L. Abelmann, M. Odijk, W. Olthuis, B. M. Weckhuysen, A. van 
den Berg, Angew. Chem. Int. Ed. 2018, 57, 10589-10594.

[11] M. E. Z. Velthoen, H. Meeldijk, F. Meirer, B. M. Weckhuysen, Chem. Eur. J. 2018, 24, 11944-11953.

[12] J. L. Palmer, E. B. Cornelius, Appl. Catal. 1987, 35, 217-235.

[13] F. Meirer, S. Kalirai, D. Morris, S. Soparawalla, Y. Liu, G. Mesu, J. C. Andrews, B. M. Weckhuysen, Sci. Adv. 2015, 1, el400199.

[14] G. R. Dyrkacz, L. Ruscic, C. L. Marshall, W. Reagan, Energy \& Fuels 2000, 71, 849-854.

[15] Z. Ristanovic, J. P. Hofmann, G. De Cremer, A. V. Kubarev, M. Rohnke, F. Meirer, J. Hofkens, M. B. J. Roeffaers, B. M. Weckhuysen, J. Am. Chem. Soc. 2015, 137, 6559-6568.

[16] M. B. J. Roeffaers, B. F. Sels, H. Uji-i, B. Blanpain, P. L’Hoest, P. A. Jacobs, F. C. De Schryver, J. Hofkens, D. E. De Vos, Angew. Chem. Int. Ed. 2007, 119, 1736-1739.

[17] M. M. Kerssens, C. Sprung, G. T. Whiting, B. M. Weckhuysen, Microporous Mesoporous Mater. 2014, 189, 136-143.

[18] L. R. Aramburo, S. Wirick, P. S. Miedema, I. L. C. Buurmans, F. M. F. De Groot, B. M. Weckhuysen, Phys. Chem. Chem. Phys. 2012, 14, 6967-6973.

[19] Z. Ristanovic, M. M. Kerssens, A. V. Kubarev, F. C. Hendriks, P. Dedecker, J. Hofkens, M. B. J. Roeffaers, B. M. Weckhuysen, Angew. Chem. Int. Ed. 2015, 54, 1836-1840.

[20] M. H. F. Kox, E. Stavitski, B. M. Weckhuysen, Angew. Chem. Int. Ed. 2007, 46, 3652-3655.

[21] J. P. Hofmann, B. M. Weckhuysen, L. R. Aramburo, J. Ruiz-Martínez, Catal. Sci. Technol. 2013, 3, 12081214.

[22] Z. Ristanovic, J. P. Hofmann, G. De Cremer, A. V. Kubarev, M. Rohnke, F. Meirer, J. Hofkens, M. B. J. Roeffaers, B. M. Weckhuysen, J. Am. Chem. Soc. 2015, 137, 6559-6568.

[23] K. Janssens, in Handb. Spectrosc. (Eds.: G. Gauglitz, D.S. Moore), Wiley-VCH, Weinheim, 2014, pp. 451508.

[24] D. Bradley, G. Roth, J. Graph. Tools 2007, 12, 13-21.

[25] F. Meyer, Signal Processing 1994, 38, 113-125.

[26] S. C. T. van der Sanden, A Fundamental Study of Spray Drying Fluid Catalytic Cracking Catalyst, Eindhoven University of Technology, 2003.

[27] M. N. M. Fl. Science, "ECAT FCC Catalyst - DCR (Fresh)," can be found under https://mfix.netl.doe.gov/ material/ecat-fcc-catalyst-dcr-fresh/, n.d.

[28] C. J. Plank, E. J. Rosinski, W. P. Hawthorne, Ind. Eng. Chem. Prod. Res. Dev. 1964, 3, 165-169.

[29] S. Kalirai, P. P. Paalanen, J. Wang, F. Meirer, B. M. Weckhuysen, Angew. Chem. Int. Ed. 2016, 55, 1113411138.

[30] Y. Liu, F. Meirer, C. M. Krest, S. Webb, B. M. Weckhuysen, Nat. Commun. 2016, 7, 12634.

[31] H. J. Jeon, S. K. Park, S. I. Woo, Appl. Catal. A Gen. 2006, 306, 1-7.

[32] L. Cedeno Caero, L. C. Ordónez, F. Pedraza, J. Ramírez, F. Pedraza, Catal. Today 2005, 108, 657-662.

[33] J. Biswas, I. E. Maxwell, Appl. Catal. 1990, 63, 197-258.

[34] S. Kalirai, U. Boesenberg, G. Falkenberg, F. Meirer, B. M. Weckhuysen, ChemCatChem 2015, 7, 36743682.

[35] E. L. Kugler, D. P. Leta, J. Catal. 1988, 395, 387-395.

[36] E. Plessers, I. Stassen, S. P. Sree, K. P. F. Janssen, H. Yuan, J. Martens, J. Hofkens, D. De Vos, M. B. J. Roeffaers, ACS Catal. 2015, 5, 6690-6695. 



\section{Chapter 4 Development of a Droplet Microreactor for the High-Throughput Fluorescence- Based Detection of Single Fluid Catalytic Cracking Particle Activity}

In this Chapter, we report on the development of a high-throughput, fluorescence-based droplet microreactor system for measuring the activity of individual Fluid Catalytic Cracking (FCC) equilibrium catalyst (ECAT) particles. An oligomerization reaction of 4-methoxystyrene, catalyzed by the $\mathrm{Br} \phi$ nsted acid sites of the ECAT particles, is performed on-chip at $95^{\circ} \mathrm{C}$. The fluorescence signal generated by the formed fluorescent reaction product inside the ECAT particles is detected near the outlet of the chip. In total 1000 ECAT particles are detected at an average rate of 1 particle every $2.4 \mathrm{~s}$. The measured fluorescence intensities show a clear activity distribution among the ECAT particles with the majority of the particles showing average to low activity and a minority of highly active particles present in the bulk mixture. The latter are of high interest as they could reveal interesting new physicochemical properties on why they are still so acidic and reactive.

This work is adapted and reproduced from the following articles:

J.C. Vollenbroek*, A.-E. Nieuwelink*, R.G. Geitenbeek*, J.G. Bomer, R.M. Tiggelaar, A. Meijerink, A. van den Berg, B.M. Weckhuysen, M. Odijk, Design and characterization of a microreactor for monodisperse catalytic droplet generation at both elevated temperatures and pressures, Nano/Micro Engineered Materials Systems (NEMS), 2017, 746-751.

J.C. Vollenbroek*, A.-E. Nieuwelink*, J.G. Bomer, R.M. Tiggelaar, A. van den Berg, B.M. Weckhuysen, M. Odijk, Droplet microreactor for reaction monitoring at elevated temperatures and pressure, In MicroTAS: 22nd International Conference on Miniaturized Systems for Chemistry and Life Sciences November 11-15, 2018, Kaohsiung, Taiwan (pp. 2131-2133).

*These authors contributed equally. 


\subsection{Introduction}

The physicochemical characterization of Fluid Catalytic Cracking (FCC) equilibrium catalyst (ECAT) particles is currently done in a bulk fashion, from which only ensemble average properties, such as acidity, pore accessibility and metal poisoning amount, can be obtained. However, due to the large age distribution in typical spent ECAT catalysts, they have a large inter-particle heterogeneity. ${ }^{[1,2]} \mathrm{Up}$ until now, the analysis of single catalyst particles has been focused on the microscopic level. For example, a lot of research has been done elucidating the deactivation process of FCC particles. The deposition of metals, such as $\mathrm{Fe}$ and $\mathrm{Ni}$, as well as the change in pore structure, acidity and accessibility have been reported with a high spatial resolution, even down to the nanometer scale thanks to the use of X-ray microscopy (XRM) as well as single-molecule fluorescence (SMF) microscopy. ${ }^{[3,4]}$ It is of course very important to gain such in-depth information ${ }^{[2]}$, but it is also very time consuming to perform all these single catalyst measurements. Therefore, these extensive analyses were only performed on a handful of ECAT particles, hampering the statistical relevance of the employed characterization methods. Due to the large inter-particle heterogeneity of an ECAT sample, there is a clear need for a fast screening of single catalyst particles to give more insight in this heterogeneous sample. By using microfluidics for the high-throughput analysis of catalyst particles, many catalyst particles can be tested more easily, which gains not only information on the single particle level, but also gives a statistical value to these measurements.

As the catalytic activity of FCC particles is linked to the availability of (Bronsted) acid sites in their zeolite domains, the activity of FCC particles can be studied with various probe molecules, such as thiophene, furfuryl alcohol and styrene. These molecules can form fluorescent oligomers in the zeolite domains of the FCC particles, which can be easily detected by (confocal) fluorescence microscopy (CFM) ${ }^{[5]}$ and UV-vis microspectroscopy ${ }^{[6]}$. More specifically, the oligomerization of styrene derivatives, such as 4-methoxystyrene, is acid-catalyzed and occurs at temperatures between 100 and $200^{\circ} \mathrm{C}$. ${ }^{[7]}$ In previous work from our group, these two micro-spectroscopic optical techniques have been combined to investigate the acidity of ECAT particles, as well as FCC particles deactivated via various laboratory-based deactivation methods with various styrene-like probe molecules. ${ }^{[7]}$ Furthermore, it has been possible to distinguish zeolite USY-based FCC particles from zeolite ZSM-5 based FCC particles. Zeolite USY and ZSM-5 have a different pore structure, as was described in Chapter 1, the general introduction. Due to this difference in pore size, different styrene oligomers will be formed inside these pores during staining. This difference is expressed as a different color (and thus a different UVvis absorption) for FCC particles with USY or ZSM-5. ${ }^{[8]}$

As discussed in Chapter 1, microfluidics has already been used for single-cell analysis at high-throughput. ${ }^{[9-11]}$ Using droplets as unique reaction vessels ${ }^{[12,13]}$, it should be possible to encapsulate single ECAT particles within droplets. Using a microfluidic system with integrated microheaters and temperature sensors ${ }^{[14-23]}$ the reaction conditions can be rapidly tuned and screened and hence it should be possible to conduct the styrene 
oligomerization reaction within the microreactor. Therefore, in this chapter we present a microfluidic platform dedicated to single catalyst particle diagnostics. ECAT particles are captured within paraffin oil droplets and flushed through a microreactor at $95{ }^{\circ} \mathrm{C}$. In-situ oligomerization of 4-methoxystyrene, present inside the catalyst particles, forms a fluorescent reaction product, which can be detected with spectroscopy. The availability of Bronsted acid sites in a catalyst particle can be linked to the amount of fluorescent reaction product and thus fluorescence signal. At the end of the microreactor channel, the fluorescent intensity is measured for all passing ECAT particles, thereby providing enough statistical information to assess the distribution of acidity within a large fraction of catalyst particles.

\subsection{Experimental Section}

\subsubsection{Microreactor Design}

To obtain the elevated temperature range that some reactions require, a microreactor can either be heated externally or internally. External heating methods rely on producing heat outside the microreactor using for example Peltier elements ${ }^{[24]}$, externally heated fluids ${ }^{[25]}$, or placing the whole system in an oil bath ${ }^{[26]}$. Integrated heating techniques include exploiting endo- and exothermic processes ${ }^{[27]}$, microwave heating ${ }^{[28-30]}$, and Joule heating ${ }^{[31-35]}$. Joule heating has the advantage of fast temperature cycling, steep gradients, and localized heating. By locating thin film metallic structures in close proximity to the microfluidic channels, the channels can be heated efficiently via Joule heating by applying a voltage over these metal microstructures. ${ }^{[15-17,19,21,23,36-38]}$ These thin film microheaters have been widely reported in the literature, and vary from use in biological assays such as PCR ${ }^{[17,19,21]}$, to microreactors for single phase purposes ${ }^{[1,37,38]}$, and finally to reactions inside droplets on a microreactor. ${ }^{[18,26]}$ When creating these thin film structures out of platinum, the heaters are stable up to at least $500{ }^{\circ} \mathrm{C}^{[14,15]}$, provided that an appropriate adhesion layer is used. Furthermore, metallic thin film structures can also be used to measure the temperature. Dedicated thin film temperature sensor structures of platinum ${ }^{[35]}$, nickel ${ }^{[39]}$ or gold ${ }^{[40]}$ can be used to measure the temperature of a microfluidic device by exploiting the temperature-dependent resistance of the metal used and are known for their high stability and sensitivity. This type of sensors is therefore called Resistance Temperature Detectors (RTDs). By monitoring the resistance of these structures, temperature read-outs can be achieved in an accurate, reproducible and fast manner. Measuring the temperature close to the heater can act as a feedback mechanism for temperature control in the microreactor. ${ }^{[14,15,22]}$

The design of the microreactor under study is illustrated in Figure 4.1a. It has several features, such as a reaction zone and optical window (b), a droplet generator (c), and a temperature sensor section (d). The dispersed phase flows into the microreactor via inlet I2, and the continuous phase via inlet I1. The heater section contains three separate heaters. Heater 1 and heater 2 ( $\mathrm{H} 1$ and $\mathrm{H} 2$ ) can be controlled separately via electrodes E9 and E2. Both these heaters have an interlocked temperature sensor, by means of 


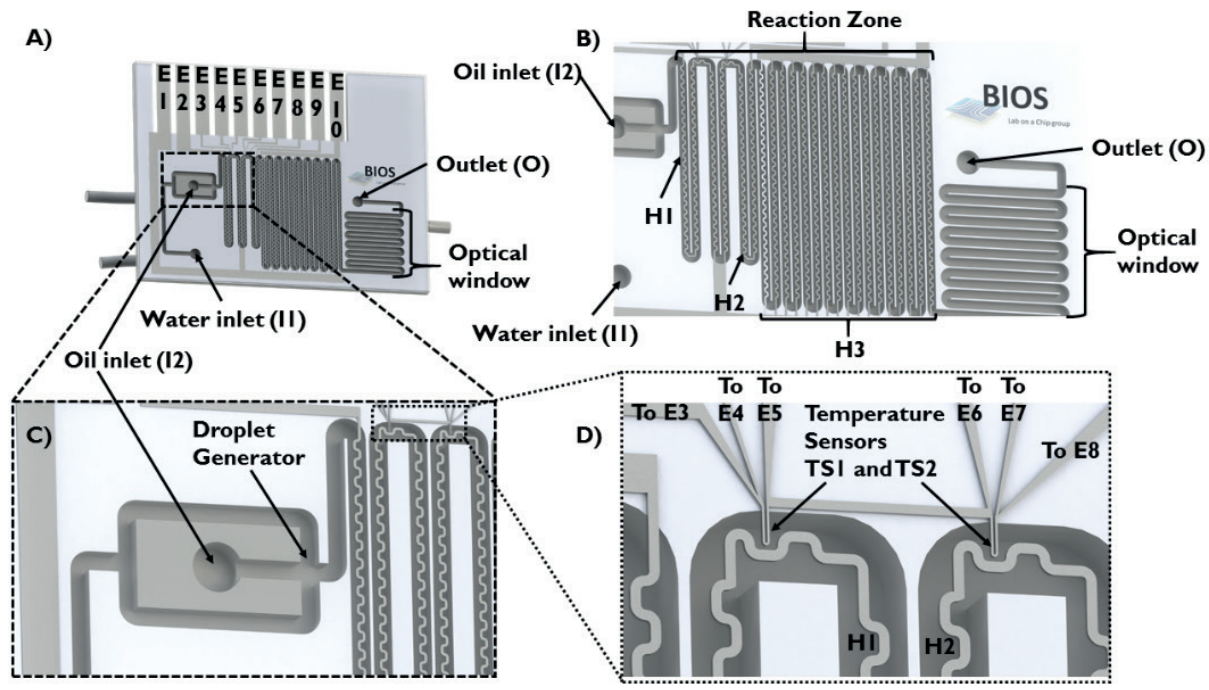

Figure 4.1: Overview of the droplet microreactor developed in this work. Channels have a width of $200 \mu \mathrm{m}$ and depth of $150 \mu \mathrm{m}$, heaters are $45 \mu \mathrm{m}$ wide and $200 \mathrm{~nm}$ thick, and the temperature sensor has a width of $10 \mu \mathrm{m}$ and thickness of $200 \mathrm{~nm}$. All metallic thin film structures are made from Pt, with a $10 \mathrm{~nm}$ tantalum adhesion layer. The bottom substrate is silicon and the top substrate is glass. Furthermore, the image contains images of (a) the contact electrodes (E1-E10) for the heater and sensor structures, flow channel layout, inlets for the dispersed phase (I1) and continuous phase (I2), and the outlet (O). b) Close-up of the reaction zone showing the three heaters $(\mathrm{H} 1-\mathrm{H} 3)$ that can be controlled via electrodes E2 (H1), E9 (H2), and E10 (H3). c) Close-up of the droplet generator, i.e. showing the flow focusing junction and the inlet for the dispersed phase. d) Close-up of the temperature sensor structures (TS1 and TS2) and the electrodes used for addressing those structures $(\mathrm{E} 3-\mathrm{E} 8)$.

which their temperature can be monitored and controlled via LabVIEW software. These temperature sensors are shown in Figure 4.ld. The optical window can be used for fluorescence measurements on the droplets, although the heater width is small enough to monitor the droplets underneath the heaters as well. A four-point measurement, in which a current is applied to electrodes E3 and E8, thereby inducing a voltage drop over the narrow sensing structure, is used to measure the resistance of the sensor. This voltage can be measured between electrodes E4 and E5 for TS1, and electrodes E6 and E7 for TS2. When the temperature increases, the resistance of the sensor increases, resulting in an increasing voltage across the specified electrodes. The final heater section, Heater 3 (H3), is a block of parallel heaters. These heaters do not have a dedicated temperature sensor. The parallel heaters are connected to E10; E1 is the ground electrode to which all heaters are connected. The parallel heater section, as well as the meandering flow channel is necessary to allow sufficient residence time at elevated temperature and pressure for chemical reactions to occur. The overall dimensions of the microreactor are $15 \mathrm{~mm} \times 20 \mathrm{~mm} \times 1 \mathrm{~mm}$.

\subsubsection{Fabrication}

The microreactors are fabricated in the cleanroom of the MESA+ NanoLab at the University of Twente. Figure 4.2 shows an overview of the executed process steps. The microreactor is a stack of a $500 \mu \mathrm{m}$ P-type $<100>$ silicon substrate, and a $500 \mu \mathrm{m}$ Mempax glass substrate. A three-mask process is used to develop the desired features both in 


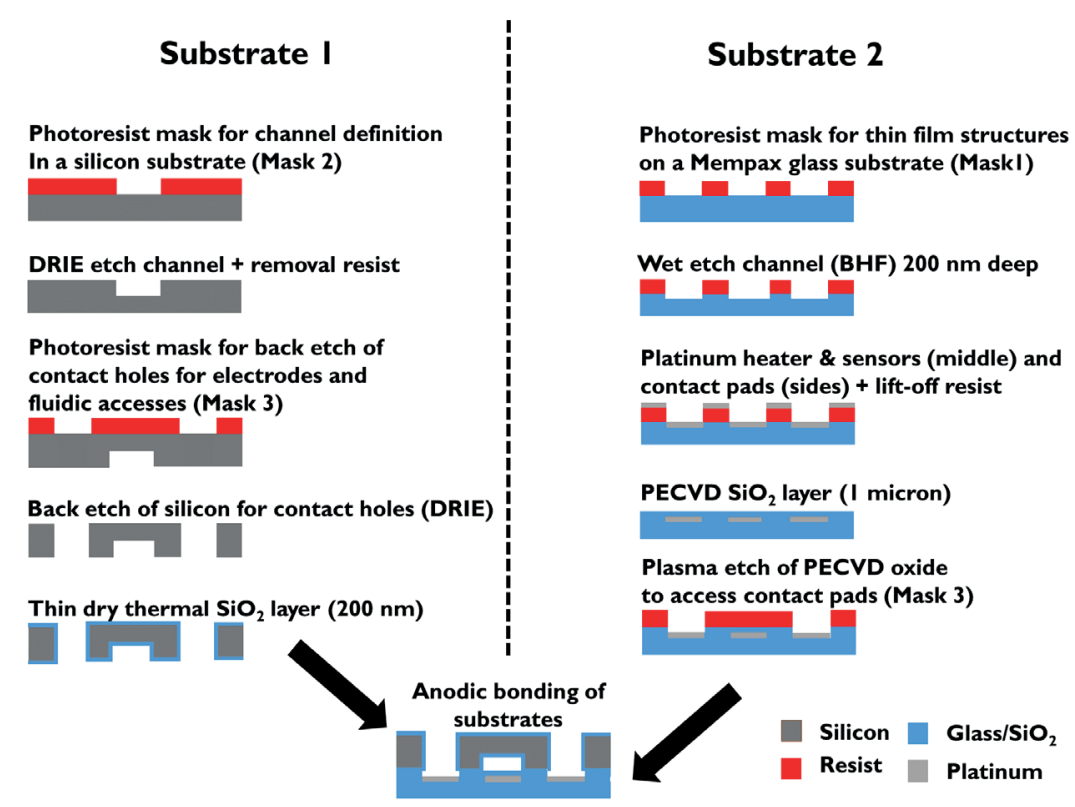

Figure 4.2: Schematic cross-sectional representation of the various steps used to fabricate the silicon/glass droplet microreactor.

and on the substrates. Using Buffered HF (BHF) as a wet etchant, the pattern for the Pt structures is etched $200 \mathrm{~nm}$ deep into the glass substrate. This process is directly followed by the deposition of a $10 \mathrm{~nm}$ thick tantalum (Ta) adhesion layer and a $190 \mathrm{~nm}$ thick $\mathrm{Pt}$ layer, thus the heaters and temperature sensors are embedded into the glass substrate. Next, the complete glass substrate containing the Pt structures is covered with $1 \mu \mathrm{m} \mathrm{SiO}_{2}$ deposited with Plasma Enhanced Chemical Vapour Deposition (PECVD). The $\mathrm{SiO}_{2}$ is then selectively removed from the contact electrodes, using standard photolithography and Reactive Ion Etching (RIE). The channels are etched $150 \mu \mathrm{m}$ deep into the silicon substrate using the Bosch process for Deep Reactive Ion Etching (DRIE).

Fluidic and electronic access holes are etched from the backside of the silicon substrate again using DRIE etching through the silicon. This ensures accessibility to both the electrodes embedded in glass, and the flow channels after the substrates are bonded together. The silicon substrate is dry oxidized, to create a $200 \mathrm{~nm}$ thin insulating $\mathrm{SiO}_{2}$ film on the walls of the channel. After completion of all the process steps, the silicon and glass substrates are anodically bonded, and the individual chips are created by means of dicing. The fabrication result is shown in Figure 4.4 where the silicon/glass droplet microreactor is shown with the designed features such as, temperature sensors, droplet generator, inlets, heaters, optical window, and outlet.

\subsubsection{Temperature Validation using Luminescent Nanoparticles}

To validate the temperature inside the channels of the microreactor, bandshape luminescence can be used ${ }^{[41,42]}$ as a ratiometric spectroscopic technique to accurately monitor temperature non-invasively. This type of luminescence thermometry exploits 
the changes in the intensity ratio of two or more emission peaks with temperature. ${ }^{[43,44]}$ This technique has been described in great detail by Geitenbeek et al. ${ }^{[45,46]}$ Luminescence measurements using a fiber probe are performed using a MDL-III-980nm-500mW laser. Upon excitation, light was collected via a fiber patch chord and a shortpass filter to the OceanOptics QEPro CCD detector. During the experiments a single-phase (1-octadecene) continuous flow of $50 \mu \mathrm{L} / \mathrm{min}$ is used. Three experiments are performed where the temperature is cycled from $70{ }^{\circ} \mathrm{C}$ to $120^{\circ} \mathrm{C}$ and back in steps of $10{ }^{\circ} \mathrm{C}$. Between the heating and cooling, sufficient time is taken for the temperature to stabilize. For heating up this is $5-10 \mathrm{~s}$ between the steps, and for cooling down this was $30 \mathrm{~s}$, because the chip is not actively cooled. The temperature is monitored using both the integrated platinum temperature sensors (TS1 and TS2) as well as the $\mathrm{NaYF}_{4}: \mathrm{Er}^{3+}, \mathrm{Yb}^{3+} \mathrm{NPs}$ dispersed in 1-octadecene (90\% ODE from Agros Organics). First, the output of the two types of temperature sensing techniques are compared by monitoring temperatures using both the integrated temperature sensor TS2 and the luminescence output at the same location. Subsequently, another temperature measurement at a location further downstream from TS2 is measured using luminescence thermometry and compared with TS2. The orange circles in Figure 4.3a and b indicate the measurement locations for luminescence thermometry. The spot size of the excitation laser, using simple fiber optics is ca. $1 \mathrm{~mm}$.

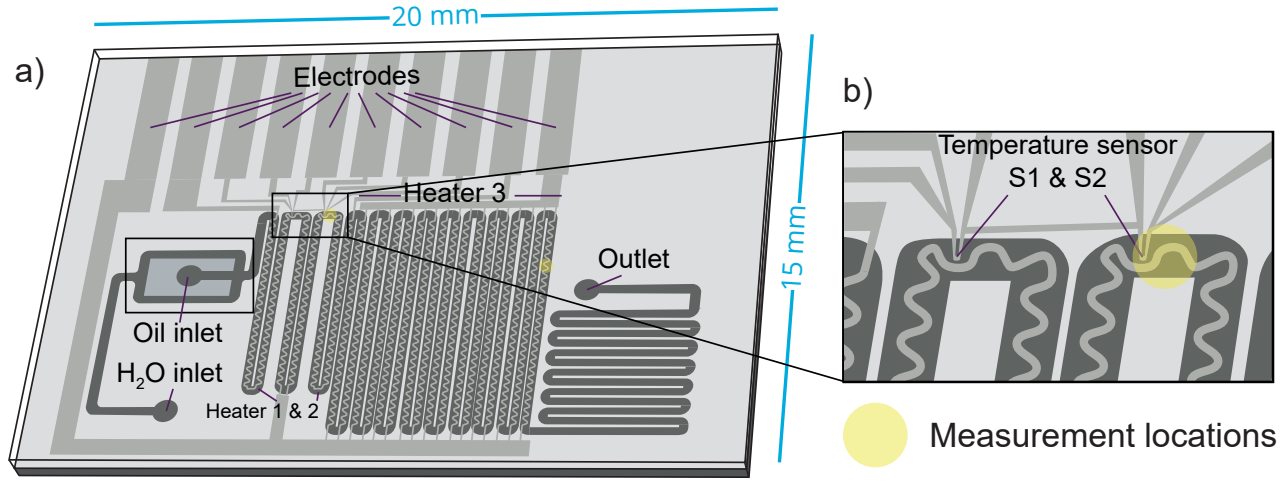

Figure 4.3: a) Schematic overview of the microreactor and temperature measurement locations, at the location of TS2 and towards the outlet, for on-chip heating and temperature sensing. b) Close-up view of TS1 and TS2 indicating the detection spot.

\subsubsection{Microreactor Setup and Experimental Conditions}

For the staining experiments, a silicon/glass droplet microreactor with a channel width of $200 \mu \mathrm{m}$ and channel height of $150 \mu \mathrm{m}$ was used. The device is shown in Figure 4.4 and the point at which the fluorescent intensity is measured is highlighted with a yellow circle. Measurements at $30{ }^{\circ} \mathrm{C}, 70{ }^{\circ} \mathrm{C}$, and $95^{\circ} \mathrm{C}$ are performed in order to determine the optimum reaction temperature, and to make sure that the reaction does not already occurs at room temperature inside the syringe. Droplets are created using a Nemesys (Cetoni $\mathrm{GmbH}$, Korbussion, Germany) syringe pump fitted with glass Hamilton syringes of $1000 \mu \mathrm{L}$ for the droplet phase (paraffin oil with particles and reactant) and $500 \mu \mathrm{L}$ for the continuous phase (FC-40 oil). The pump is controlled by Nemesys software and the syringes are connected to the chip using fused silica tubing (Polymicro Technologies, 


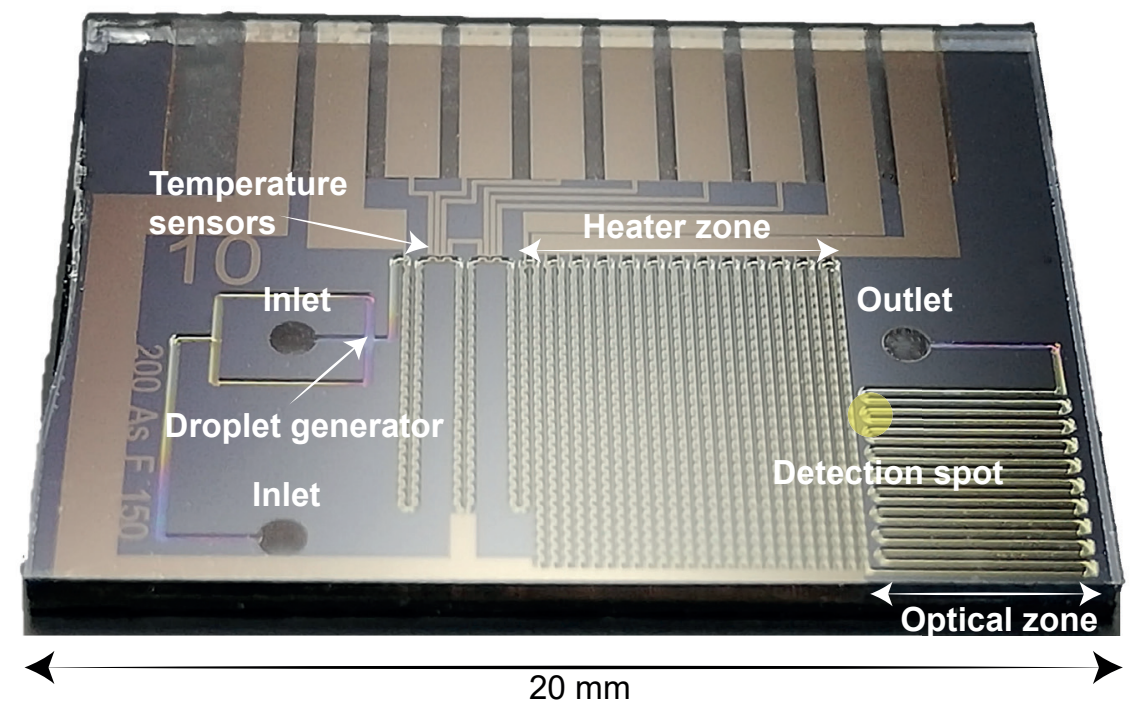

Figure 4.4: Droplet microreactor used for the experiment, with various features highlighted, including the temperature sensors, droplet generator, inlets, outlet, and the detection spot for fluorescence detection (indicated by the yellow square).

ID $=250 \mu \mathrm{m}, \mathrm{OD}=360 \mu \mathrm{m}$ ) and connectors (NanoPort ferrule: N-123-03, NanoPort nut: F-123H, MicroTight connector: P-882) from Idex-HS. Experiments at high pressure are performed by adding a P786 (5 bar) backpressure regulator from Idex-HS to the setup. The channel walls are made hydrophobic by flushing the flow channel with a solution of $5.55 \mu \mathrm{L}$ tridecafluoro-1,1,2,2-tetrahydrooctyltrichlorosilane (FOTS, Sigma Aldrich) added to $1.5 \mathrm{~mL} \mathrm{FC-40} \mathrm{(Fluorinert} \mathrm{3M)} \mathrm{oil.} \mathrm{The} \mathrm{microreactor} \mathrm{is} \mathrm{flushed} \mathrm{for} 45$ minutes with the FOTS and FC-40 solution and then for 45 minutes with only FC-40. For the experiments flow rates of $5 \mu \mathrm{L} / \mathrm{min}$ for the droplet phase and $9 \mu \mathrm{L} / \mathrm{min}$ for the continuous phase are used, thus a total flow rate of $14 \mu \mathrm{L} / \mathrm{min}$. The total length of the channel is 32.3 $\mathrm{cm}$ and the velocity of the droplets is $8.3 \mathrm{~mm} / \mathrm{s}$. The measurement point is at $30.7 \mathrm{~cm}$ after the droplet generator, resulting in a residence time of 37 seconds per droplet from the point of generation till the detection spot.

\subsubsection{Catalyst Characterization and Related Setup}

From bulk measurements with a Nikon Eclipse 90i confocal fluorescence microscope with an AlR scan head (10x objective) equipped with a $488 \mathrm{~nm}$ argon ion liquid state Melles Griot laser ( $40 \mathrm{~mW}$ ), it was found that the reaction products have an emission maximum at $600 \mathrm{~nm}$ when $488 \mathrm{~nm}$ is used as excitation wavelength. A batch test of the reaction was done in which the formation of fluorescent product upon the acid-catalyzed oligomerization of 4-methhoxystyrene is monitored over $5 \mathrm{~min}$ at $100{ }^{\circ} \mathrm{C}$. In Figure 4.5 the proposed mechanism is shown for the oligomerization of styrene derivatives. [5,47] The fluorescent signal of the particles is shown after 0,2 , and 5 min respectively. Some particles form a lot of fluorescent product while others do not show a signal at all. An ECAT sample is calcined for $5 \mathrm{~h}$ at $600{ }^{\circ} \mathrm{C}\left(1{ }^{\circ} \mathrm{C} / \mathrm{min}\right.$ to $120^{\circ} \mathrm{C}$, hold for $1 \mathrm{~h}$, 
a)

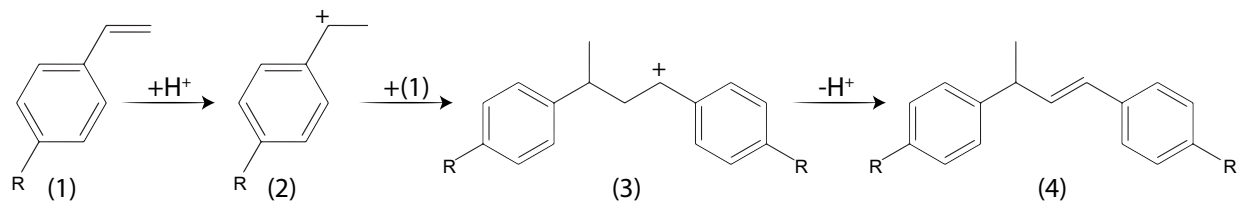

b) $0 \mathrm{~min}$

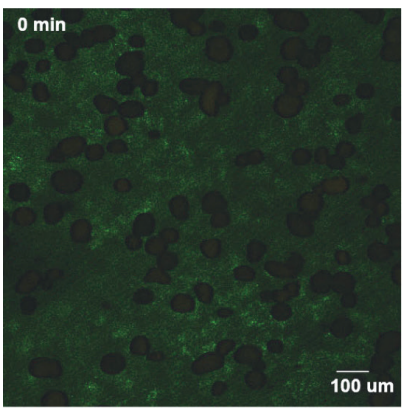

$2 \mathrm{~min}$

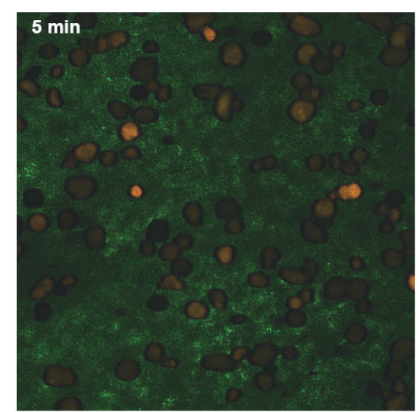

Figure 4.5: Mechanism of the oligomerization of styrene derivatives as proposed by [47]. Oligomerization can continue or cyclization of (4) can occur. From (3) the molecules are fluorescent when excited with $\lambda=488$ $\mathrm{nm}$ b) Test results of the fluorescent oligomerization reaction of 4-methoxystyrene inside ECAT particles in paraffin oil at three time intervals $(0,2$, and $5 \mathrm{~min})$ at $100^{\circ} \mathrm{C}$ as measured with fluorescence microscopy. The contrast and brightness have been enhanced using Adobe Photoshop for visibility purposes only.

then $5{ }^{\circ} \mathrm{C} / \mathrm{min}$ ) to remove all residual coke species. A sieve fraction of $38-75 \mu \mathrm{m}$ is used for further experiments. $6 \mathrm{mg}$ of calcined ECAT particles is impregnated with $10 \mu \mathrm{L}$ 4-methoxystyrene (Sigma Aldrich 97\%). $2 \mathrm{~mL}$ of paraffin oil (Sigma Aldrich analytical grade) is added to the reactant/particle mixture and after shaking, such that a suspension is created, the oil/reactant/particle mixture is put into a $1000 \mu \mathrm{L}$ glass Hamilton syringe. The paraffin oil is heated up to $100{ }^{\circ} \mathrm{C}$ for $5 \mathrm{~h}$ to remove volatile compounds that cause gas bubbles in the microreactor around $90^{\circ} \mathrm{C}$. Due to the size of the particles, precautions had to be taken to keep them in suspension, because otherwise gravity causes them to sink to the bottom of both the syringe and capillary. Therefore, a Teflon coated magnetic stir bar is added inside the syringe to keep the particles in suspension.

Moreover, a vibrational motor (Mouser Electronics) is mounted to the tip of the syringe to shake the tubing and to prevent the particles from getting stuck in the glass capillary. Both the magnetic stir bar and vibrational motor are shown in a schematic representation and in an image of the actual setup in Figure 4.6. As an extra precaution against particle sedimentation the syringes are positioned vertically.

\subsubsection{Fluorescence Microscopy Setup}

A Leica DMi 5000 M inverse microscope with 10x objective, equipped with a Hg lamp and a BGR filter cube is used for the fluorescence measurements. The BGR filter cube has three excitation bands $(420 \mathrm{~nm} \pm 30 \mathrm{~nm}, 495 \mathrm{~nm} \pm 15 \mathrm{~nm}, 570 \mathrm{~nm} \pm 20 \mathrm{~nm})$ and three emission bands $(465 \mathrm{~nm} \pm 20 \mathrm{~nm}, 530 \mathrm{~nm} \pm 30 \mathrm{~nm}, 640 \mathrm{~nm} \pm 40 \mathrm{~nm})$. A Hamamatsu Orca Flash4.0 V2 camera is used to capture the fluorescence images. The exposure time of the camera during filming is set to 1 millisecond and the framerate to $200 \mathrm{fps}$. 

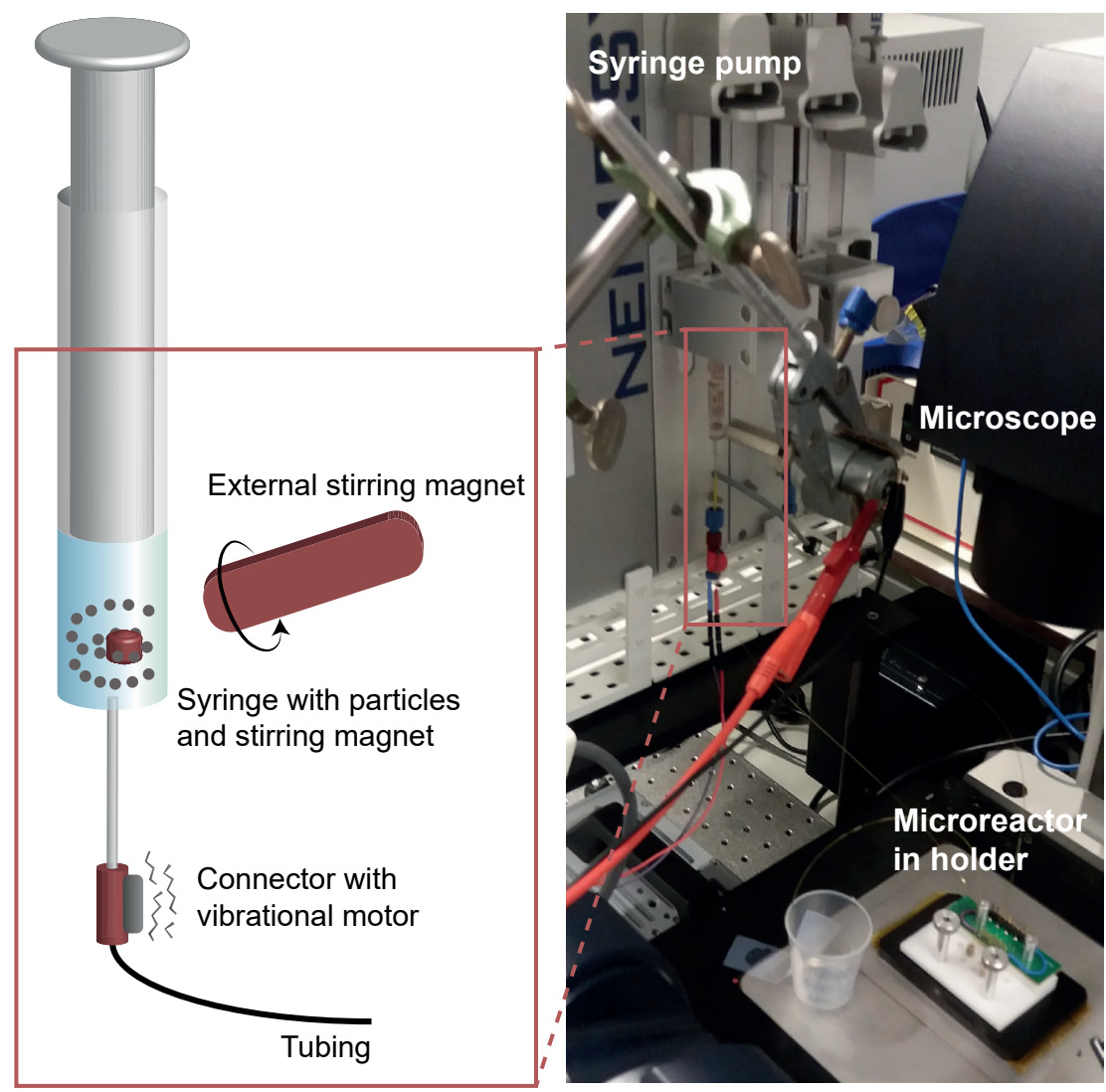

Figure 4.6: Precautions to keep the ECAT FCC particles in suspension and prevent them from sedimentation. The small stirring magnet inside the syringe, as well as the electromotor that is driving it are shown. The vibrational motor added to the tip of the syringe is also highlighted. Setup used during the activity staining experiments showing the stirring precautions (magnets, vibrational motor and vertical syringes), glass capillaries, and the holder for the microreactor.

\subsection{Results and Discussion}

\subsubsection{Temperature Validation using Luminescent Nanoparticles}

The accuracy of the Pt sensors in the microreactor was validated using the temperature dependent luminescence of $\mathrm{NaYF}_{4}: \mathrm{Er}^{3+}, \mathrm{Yb}^{3+} \mathrm{NPs}$ dispersed in 1-octadecene. This technique has proven to be very useful for determining the temperature inside a microchannel ${ }^{[46]}$. The temperature measurements are accurate $\left( \pm 1-5^{\circ} \mathrm{C}\right.$ up to $\left.\sim 400^{\circ} \mathrm{C}^{[45]}\right)$, non-invasive, not depending on probe concentration and the NPs are easily dispersed in the microchannel. Figure 4.7a shows the temperatures as determined with both techniques (Pt sensors and luminescence thermometry) by cycling the temperature between 70 and $120^{\circ} \mathrm{C}$ three times on one chip. The red diamonds are the temperatures measured with the integrated Pt thermometers while the cyan dots represent the temperatures calculated from the temperature-dependent luminescence of the NPs for sensing at the same spot (i.e. at TS2, as marked in Figure 4.3a by the left yellow circle). The temperature outputs from both techniques are plotted in Figure 4.7a and coincide within $0.34^{\circ} \mathrm{C}$ on average. The 

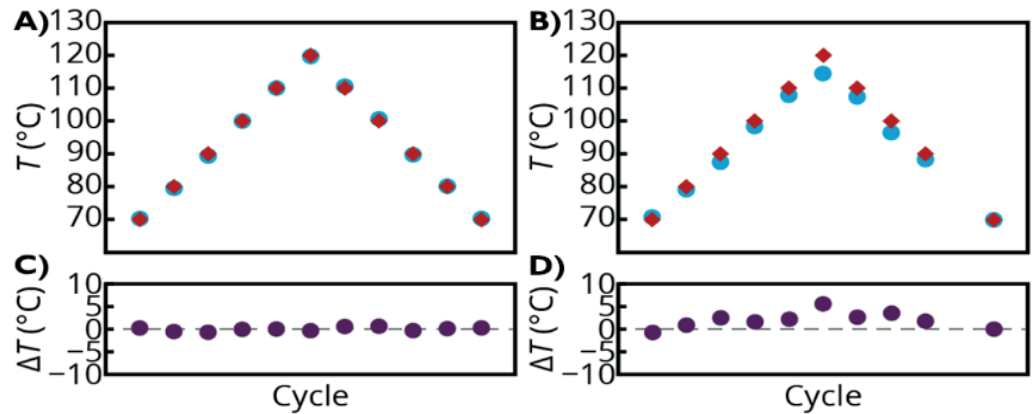

Figure 4.7: Temperature measurements in silicon-glass chip. Temperature measurements using the on-chip RTD sensors (red diamonds) and temperature-dependent luminescence from the NaYF4 NPs (cyan dots). The temperatures were determined on the same spot as the platinum sensor TS2 (a) and at the end of the chip (b), as indicated by the orange circles in Figure 4.4. The respective temperature difference measured at both positions between the platinum sensor TS2 and the NPs is plotted in c) and d).

maximum and mean discrepancies observed were $0.68^{\circ} \mathrm{C}$ and $0.34^{\circ} \mathrm{C}$, respectively. These results show that the use of the Pt sensors is a valid method, in this case, to monitor temperatures inside microfluidic channels even though the platinum sensors are located 1 $\mu \mathrm{m}$ (separated by a $\mathrm{SiO}_{2}$ layer) above the channels. Figure 4.9b shows the results obtained from both the Pt sensors and the luminescent NPs, for monitoring the luminescence output at the outlet of the microfluidic device, as indicated by the rightmost yellow circle in Figure 4.3a. Note that only heaters 1 and 2 are switched on during these experiments. The temperatures measured with the integrated temperature sensors (TS1 and TS2) are measured upon cycling three times from $70-120^{\circ} \mathrm{C}$ in steps of $10^{\circ} \mathrm{C}$ similar to the previous experiment. However, since the luminescence output is monitored at a different location in the chip, cooling down of the liquid passing through the microreactor can result in lower temperatures monitored with luminescence thermometry. This is indeed observed in Figure 4.7d) where significant temperature differences can be seen between the temperatures from the two different temperature sensing techniques. At $70{ }^{\circ} \mathrm{C}$ this effect is still minimal, however, at higher temperatures the difference increases, up to a difference of $5.6^{\circ} \mathrm{C}$ at $120^{\circ} \mathrm{C}$. However, the temperature difference is relatively small as a result of the high thermal conductance of silicon, especially considering that only $\mathrm{H} 1$ and $\mathrm{H} 2$ are used. If $\mathrm{H} 3$ is used to heat the microreactor the temperature distribution can be considered to be homogeneous over the whole chip.

\subsubsection{Droplet Stability at Various Temperatures and Pressures}

Various tests were performed on droplets being created at different conditions. In Figure 4.8, multiple images are shown of oil droplets in water as continuous phase at different temperatures: room temperature, $50{ }^{\circ} \mathrm{C}, 100{ }^{\circ} \mathrm{C}, 120{ }^{\circ} \mathrm{C}$ i.e. above the boiling point of water, and finally $120^{\circ} \mathrm{C}$ at a backing pressure of 5 bar. For all measurements, the flow rates of the water and oil phase are $15 \mu \mathrm{L} / \mathrm{min}$ and $3 \mu \mathrm{L} / \mathrm{min}$ respectively, and images were taken using the high-speed camera at a framerate of $125 \mathrm{fps}$. The width of the channel for these experiments was $250 \mu \mathrm{m}$. The volume of the droplet is approximately $46 \mathrm{~nL}$ and does not change significantly with temperature. When the temperature is increased to $100^{\circ} \mathrm{C}$, small gas bubbles start to appear in the channel, as is shown in Figure 
A) $\mathrm{T}=23^{\circ} \mathrm{C} ; \mathrm{P}=$ atmospheric pressure

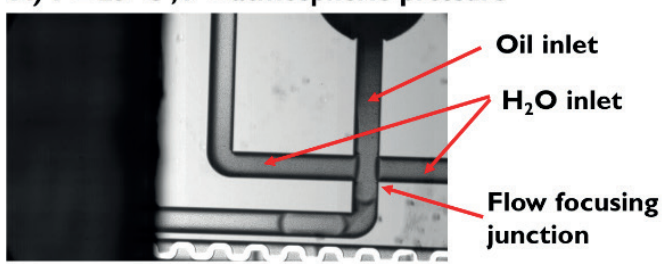

B) $\mathbf{T}=50^{\circ} \mathrm{C} ; \mathrm{P}=$ atmospheric pressure

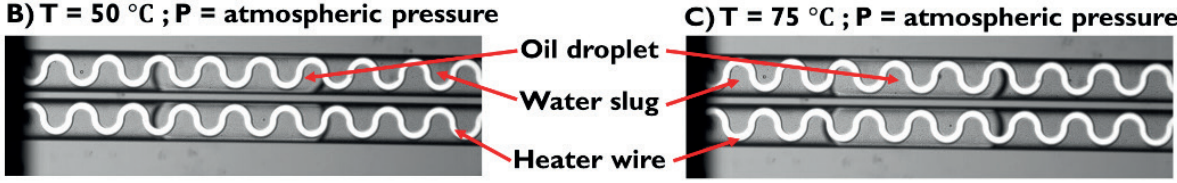

D) $\mathrm{T}=120^{\circ} \mathrm{C} ; \mathrm{P}=$ atmospheric pressure

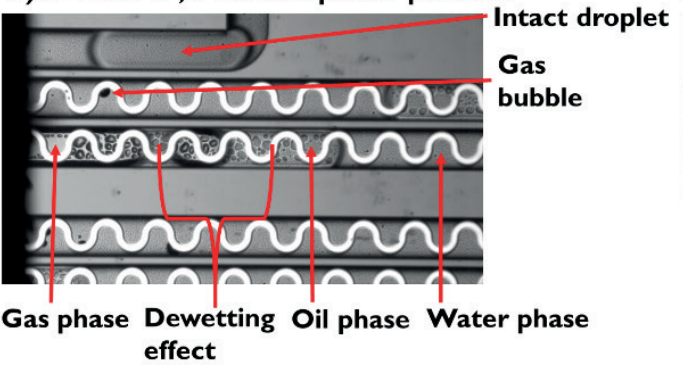

E) $\mathbf{T}=120^{\circ} \mathrm{C} ; \mathbf{P}=\mathbf{5}$ bar

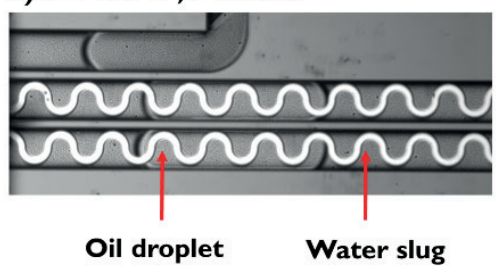

Figure 4.8: Droplets created at various conditions, where in a) the heaters are switched off, b) the temperature is kept at $50{ }^{\circ} \mathrm{C}$, c) the temperature is $100{ }^{\circ} \mathrm{C}$ and small gas bubbles are collected by the droplets, d) the temperature is above the boiling point of water (at $120^{\circ} \mathrm{C}$ ), and finally e) the temperature is above the boiling point of water $\left(\right.$ at $120^{\circ} \mathrm{C}$ ), but the system is at an outlet pressure of approximately 5 bar. The channel width is $250 \mu \mathrm{m}$.

4.8c. These bubbles find their origin underneath the heater and when they encounter an oil droplet, they show affinity towards the oil phase. Inside the oil droplet the small bubbles seem to follow the path of the heater. At $120{ }^{\circ} \mathrm{C}$, large gas bubbles are formed, and the oil droplets start to break up, showing a dewetting phenomenon at the channel ceiling. A collapsed droplet, with large gas bubbles in the droplet, is shown in Figure 4.8d. Once the backpressure regulator ( 5 bar) is added, the droplets remain intact at 120 ${ }^{\circ} \mathrm{C}$. This is shown in Figure 4.8e. The droplet monodispersity is maintained when using a backpressure regulator at the outlet.

Droplet monodispersity is important when droplets are used as chemical reactor vessels to ensure the same reaction conditions and variations in, for example, the amount of available reactant and mass transport.

\subsubsection{Reaction Temperature Determination}

Figure 4.9a shows particles at the inlet at $30^{\circ} \mathrm{C}$, where it can be seen that there is no fluorescent signal yet, and thus it can be concluded that the reaction did not occur inside the syringe. Furthermore, increasing the temperature to $70{ }^{\circ} \mathrm{C}$ does not result in a fluorescent signal near the outlet of the microreactor, as is shown in Figure 4.9b. The particle is difficult to see, but the absence of the interface between the paraffin oil droplet and the continuous phase (FC-40 oil) indicates the presence of a particle. After 
a)
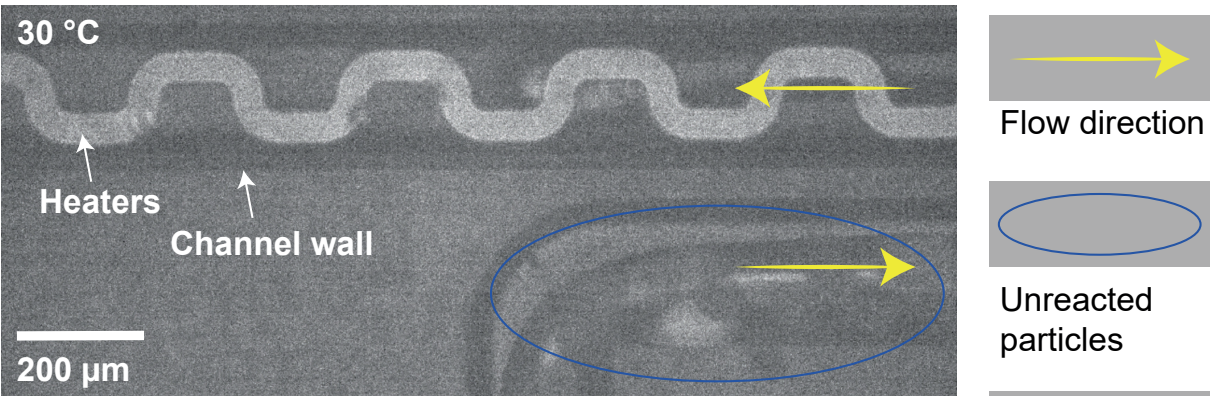

Flow direction

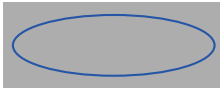

Unreacted particles

b) $70^{\circ} \mathrm{C}$
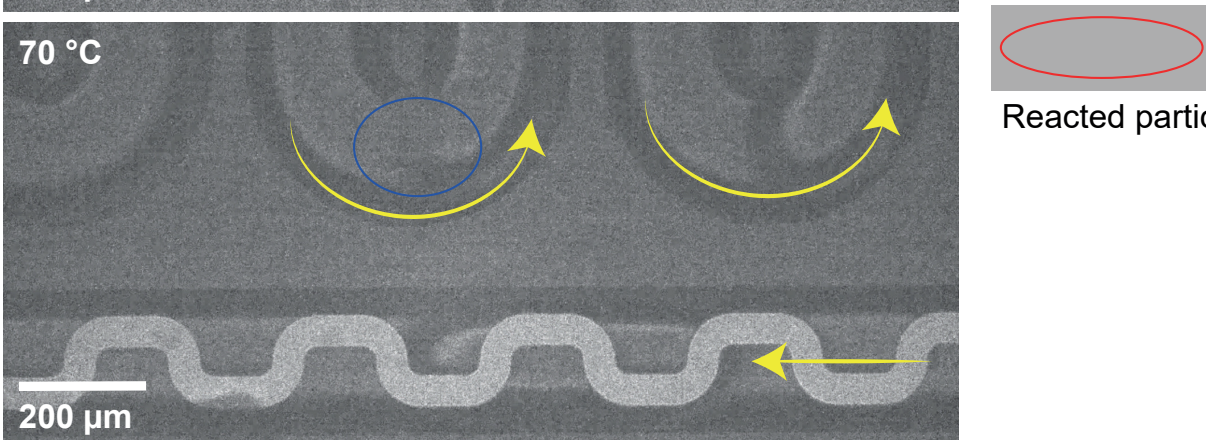

Reacted particle

c)

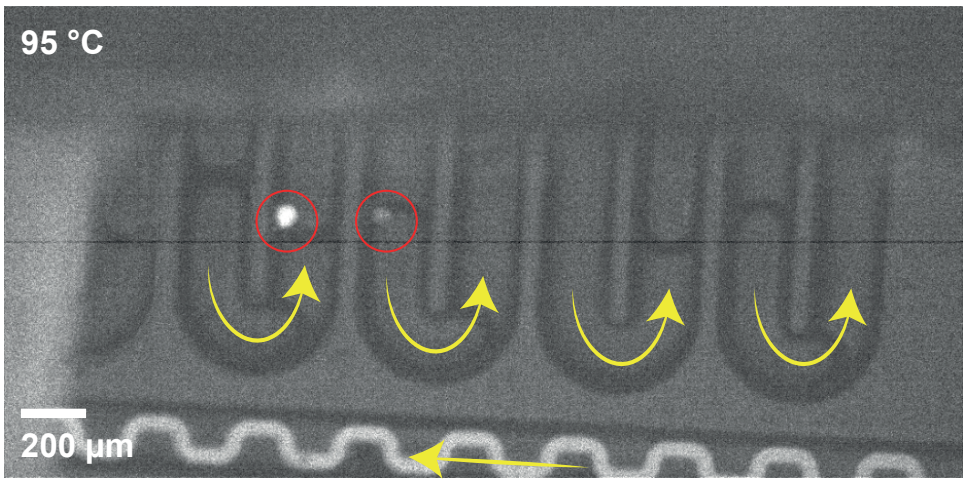

Figure 4.9: ECAT particles at various temperatures and positions in the microreactor, with a) particles at the inlet that did not react at $30^{\circ} \mathrm{C}, \mathrm{b}$ ) a particle in the optical window region near the outlet that has not reacted at a temperature of $70^{\circ} \mathrm{C}$, and c) two particles in the optical window region with different fluorescent signals after reacting in the chip at $95^{\circ} \mathrm{C}$.

increasing the temperature to $95^{\circ} \mathrm{C}$ particles show a fluorescent signal. Figure 4.9c shows two different particles, one with a high-intensity fluorescence signal and the other with a low-intensity fluorescence signal. Because no signal was seen at $70^{\circ} \mathrm{C}$, the chance that the reaction already starts inside the syringe is negligible and all the observed fluorescence at $95^{\circ} \mathrm{C}$ is caused by the residence time of the particle in the microreactor ( $37 \mathrm{~s}$ ). Therefore, it is concluded that $95^{\circ} \mathrm{C}$ is the minimal temperature necessary for the reaction to occur in the microreactor. This temperature setting will be used for the experiments in the next section.

\subsubsection{Measuring and Analyzing Fluorescent Catalyst Particles}

New series of experiments are carried out at a microreactor temperature of $95^{\circ} \mathrm{C}$, and 
the fluorescence of ECAT particles is measured in the detection spot, as indicated by the yellow dot in Figure 4.4. During the experiments a total amount of $\sim 1000$ fluorescent particles are detected at an average rate of 1 particle per 2.4 seconds. Data analysis on the movies that are recorded during the experiments is done with Matlab. Frames containing a fluorescent particle are found with an algorithm that searches the video file for those frames with a highly fluorescent region in them. After the frames are collected, another Matlab script is used to find the approximate edges of the particles. Figure 4.10a shows an example of a frame found with the Matlab script containing a particle exhibiting high fluorescent intensity. Figure $4.10 \mathrm{~b}$ shows a zoom-in window made around this particle and Figure 4.10c shows a converted image with a colormap showing the pixel intensities of the particle. Figure 4.10d shows a frame containing a particle that has less fluorescent intensity and is more difficult to distinguish from the background. Figure 4.10e and f show the zoom-in window and the colormap of this less bright particle. It is shown that this less bright particle in Figure 4.10f has its highest intensity values around 140, whereas the bright particle in Figure 4.10c has its lower intensity value around 140. The lowest intensity values for less bright particles are close to the background intensity value, which has an average value of 91 . The total span of pixel intensities is between 0 and 255, and the maximum intensity in all the particles found was 252 . This means that even for the brightest particle, the pixels are not saturated. To determine the intensity of a particle, all pixel-values within the zoom-in window above a threshold of 94 are counted and divided by the number of pixels found above this threshold value. Figure 4.11 shows a histogram of the particle intensity distribution.

The intensity difference is given on the x-axis, meaning that the lowest set of found particles (with values slightly above the threshold) is set at 0 and the intensity of the

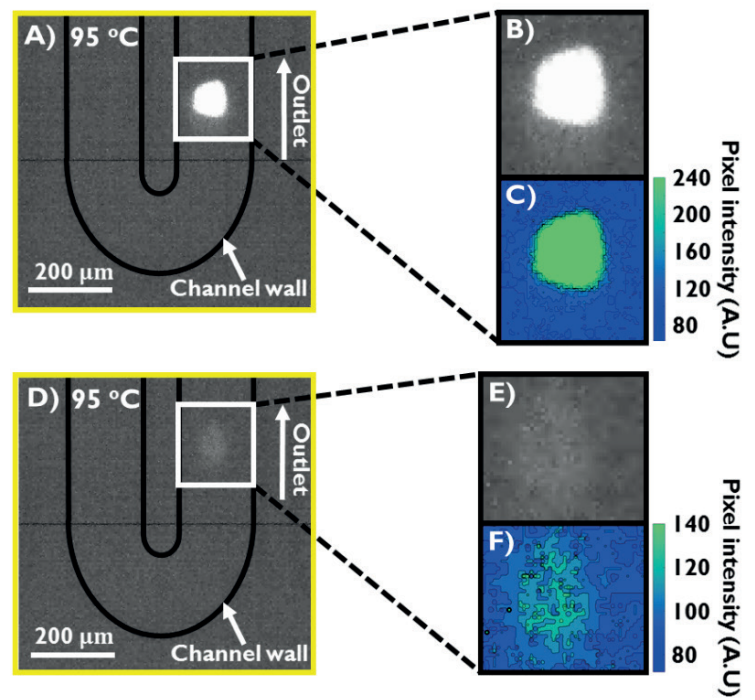

Figure 4.10: Fluorescence intensity of two Equilibrium catalyst (ECAT) Fluid Catalytic Cracking (FCC) particles at the outlet of the microreactor at $95^{\circ} \mathrm{C}$, with a) showing an ECAT particle with high fluorescent intensity and b) an ECAT particle with a lower intensity. c), d), e) and f) show a zoomed-in area and a colormap with a pixel intensity color bar of a) and b), respectively. 


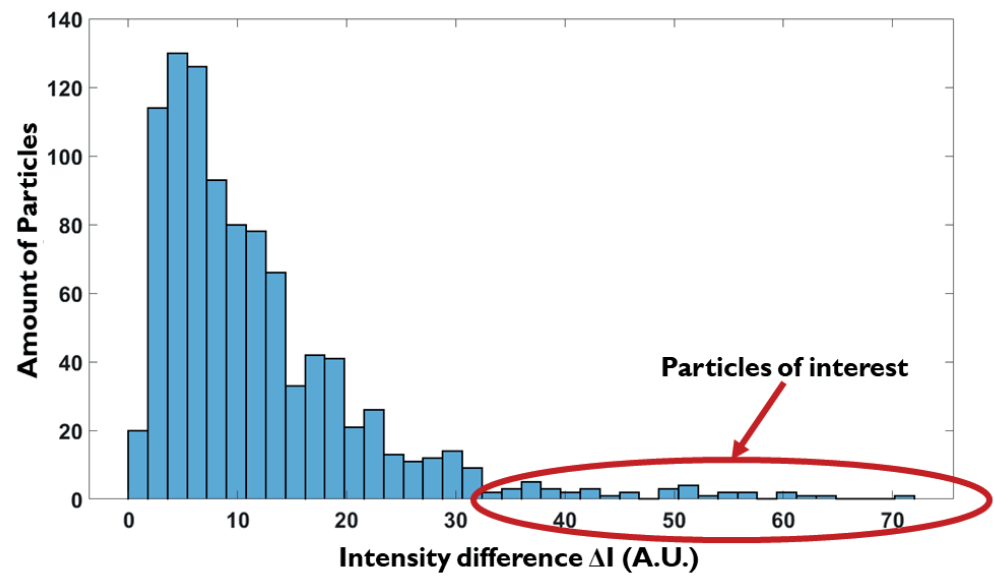

Figure 4.11: Histogram with on the $\mathrm{x}$-axis the average fluorescence intensity of a particular ECAT particle stained with 4-methoxystyrene and on the y-axis the number of FCC particles with a specific fluorescence intensity

other sets of particles is shown relatively to this lowest set. Furthermore, particles that did not react at all, as also shown in the batch test in Figure 4.5, are not distinguishable from the background signal and remain therefore undetected. The histogram in Figure 4.11 can be divided into two parts. The bulk particles showing low to average fluorescent intensity, and the extraordinary particles showing high fluorescent intensity. The threshold between these two parts lies between 30 and 40 intensity points on the $\mathrm{x}$-axis. Due to the direct correlation of the measured particle intensity to the activity of the particle, the particles on the right-hand side of the threshold are the so-called particlesof-interest. Due to the high amount of available Brønsted acid sites, leading to a high fluorescence, these particles are expected to be less deactivated by the harsh reaction conditions experienced in a commercial FCC reactor.

\subsection{Conclusions}

In this Chapter, we have designed, constructed and tested a droplet microreactor that can be used for chemical analysis at elevated temperatures and pressure. Stable formation of droplets as well as heating of the droplets up to $120^{\circ} \mathrm{C}$, at pressures up to 5 bar, has been achieved with this droplet microreactor. The generated droplets are highly monodisperse, creating well-defined and uniform reaction environments. The Pt temperature sensors accurately represent the temperature inside the channel close to the sensor, which is confirmed by measurements using $\mathrm{NaYF}_{4}: \mathrm{Er}^{3+}, \mathrm{Yb}^{3+}$ nanoparticles, which exhibit temperature-dependent luminescence behavior. These temperature measurement techniques show a maximum deviation in temperature reading close to the Pt sensor of $0.86^{\circ} \mathrm{C}$.

We have demonstrated a high-throughput droplet microreactor for the screening of individual Fluid Catalytic Cracking (FCC) particles, based on their activity. The screening was performed in-situ at elevated temperatures up to $95^{\circ} \mathrm{C}$ using a fluorescent probe reaction as marker for the catalysts' activity. We have performed an oligomerization 
reaction of 4-methoxystyrene with calcined FCC Equilibrium catalyst (ECAT) particles inside paraffin oil droplets. In total $\sim 1000$ FCC particles were detected at an average rate of 1 particle per $2.4 \mathrm{~s}$. Because the microreactor contains integrated heaters and temperature sensors, reaction conditions could be tuned, and the optimum reaction condition was found. Highly active ECAT particles show a strong fluorescent signal after a residence time of $37 \mathrm{~s}$ at $95^{\circ} \mathrm{C}$. Movies of the fluorescent FCC particles passing the detection spot were recorded and analyzed with Matlab. The data show a distribution in catalyst particle activity, displaying a clear distribution in most bulk FCC particles that give a low-to-moderate fluorescence signal and a minority of highly active ECAT particles showing a high amount of fluorescence signal; hence possessing a very high acidity. These highly-active FCC ECAT particles are the particles-of-interest as they may possess physicochemical properties being responsible for this high acidity, hence anticipated high cracking reactivity. Future work will focus on separating this group of highly active FCC ECAT particles from the bulk for further in-depth analysis.

\subsection{Acknowledgements}

Jan van Nieuwkasteele (University of Twente) is acknowledged for his help with setting up the Hamamatsu camera.

\subsection{References}

[1] E. T. C. Vogt, B. M. Weckhuysen, Chem. Soc. Rev. 2015, 44, 7342-7370.

[2] I. L. C. Buurmans, B. M. Weckhuysen, Nat. Chem. 2012, 4, 873-886.

[3] Y. Liu, F. Meirer, C. M. Krest, S. Webb, B. M. Weckhuysen, Nat. Commun. 2016, 7, 12634.

[4] Z. Ristanovic, J. P. Hofmann, G. De Cremer, A. V. Kubarev, M. Rohnke, F. Meirer, J. Hofkens, M. B. J. Roeffaers, B. M. Weckhuysen, J. Am. Chem. Soc. 2015, 137, 6559-6568.

[5] E. Stavitski, M. H. F. Kox, B. M. Weckhuysen, Chem. Eur. J. 2007, 13, 7057-7065.

[6] I. L. C. Buurmans, J. Ruiz-Martínez, W. V Knowles, D. Van Der Beek, J. A. Bergwerff, E. T. C. Vogt, B. M. Weckhuysen, Nat. Chem. 2011, 3, 862-867.

[7] L. R. Aramburo, S. Wirick, P. S. Miedema, I. L. C. Buurmans, F. M. F. De Groot, B. M. Weckhuysen, Phys. Chem. Chem. Phys. 2012, 14, 6967-6973.

[8] M. M. Kerssens, A. Wilbers, J. Kramer, P. De Peinder, G. Mesu, Faraday Discuss. 2016, 188, 69-79.

[9] S. Teh, R. Lin, L. Hung, A. P. Lee, Lab Chip 2008, 8, 198-220.

[10] X. Casadevall, Chem. Commun. 2011, 47, 1936-1942.

[11] J. H. Leamon, D. R. Link, M. Egholm, J. M. Rothberg, Nat. Methods 2006, 3, 541-543.

[12] S. T. S. Kaminski, P. Garstecki, Chem. Soc. Rev. 2017, 46, 6210-6226.

[13] L. Shui, A. Van Den Berg, J. C. T. Eijkel, Lab Chip 2009, 9, 795-801.

[14] R. M. Tiggelaar, R. G. P. Sanders, A. W. Groenland, J. G. E. Gardeniers, Sensors Actuators, A Phys. 2009 , 152, 39-47.

[15] W.-Y. Chang, Y.-S. Hsihe, Microelectron. Eng. 2016, 149, 25-30.

[16] V. Miralles, A. Huerre, F. Malloggi, M.-C. Jullien, Diagnostics 2013, 3, 33-67.

[17] J. L. Lin, M. H. Wu, C. Y. Kuo, K. Da Lee, Y. L. Shen, Biomed. Microdevices 2010, 12, 389-398.

[18] C. Fang, D. Lee, B. Stober, G. G. Fuller, A. Q. Shen, RSC Adv. 2015, 5, 85620-85629.

[19] T. Pennell, T. Suchyna, J. Wang, J. Heo, J. D. Felske, F. Sachs, S. Z. Hua, Anal. Chem. 2008, 80, 2447-2451.

[20] A. I. K. Lao, T. M. H. Lee, I. M. Hsing, N. Y. Ip, Sensors Actuators, A Phys. 2000, 84, 11-17.

[21] N. Crews, C. Wittwer, R. Palais, B. Gale, Lab Chip 2008, 8, 919-924.

[22] R. Zhong, X. Pan, L. Jiang, Z. Dai, J. Qin, B. Lin, Electrophoresis 2009, 30, 1297-1305.

[23] R. Phatthanakun, P. Deekla, W. Pummara, C. Sriphung, C. Pantong, N. Chomnawang, ECTI-CON, Annu. Int. Conf. Electr. Eng. Comput. Telecommun. Inf. Technol. Assoc. 2011, 14-17.

[24] J. Khandurina, T. E. McKnight, S. C. Jacobson, L. C. Waters, R. S. Foote, J. M. Ramsey, Anal. Chem. 2000, 72, 2995-3000. 
[25] J. Yang, Y. Liu, C. B. Rauch, R. L. Stevens, R. H. Liu, R. Lenigk, P. Grodzinski, Lab Chip 2002, 2, $179-187$.

[26] K. Olivon, F. Sarrazin, Chem. Eng. J. 2013, 227, 97-102.

[27] R. M. Guijt, A. Dodge, G. W. K. Van Dedem, N. F. De Rooij, E. Verpoorte, Lab Chip 2003, 3, 1-4.

[28] J. Geist, J. J. Shah, M. V. Rao, M. Gaitan, J. Res. Natl. Inst. Stand. Technol. 2007, 112, 177-189.

[29] J. J. Shah, J. Geist, M. Gaitan, J. Micromechanics Microengineering 2010, 20, 105025.

[30] Y. V Bykov, K. I. Rybakov, V. E. Semenov, J. Phys. D. Appl. Phys. 2001, 34, R55-R75.

[31] Z. J. Jiao, X. Y. Huang, N. T. Nguyen, J. Micromechanics Microengineering 2008, 18, 045027.

[32] Z. Jiao, X. Huang, N. T. Nguyen, P. Abgrall, Microfluid. Nanofluidics 2008, 5, 205-214.

[33] B. Selva, J. Marchalot, M. C. Jullien, J. Micromechanics Microengineering 2009, 19, 065002.

[34] D. Vigolo, R. Rusconi, R. Piazzaa, H. A. Stone, Lab Chip 2010, 10, 795-798.

[35] R. M. Tiggelaar, J. W. Berenschot, J. H. de Boer, R. G. P. Sanders, J. G. E. Gardeniers, R. E. Oosterbroek, A. van den Berg, M. C. Elwenspoek, Lab Chip 2005, 5, 326-336.

[36] A. V. Pattekar, M. V. Kothare, J. Microelectromechanical Syst. 2004, 13, 7-18.

[37] R. M. Tiggelaar, P. Van Male, J. W. Berenschot, J. G. E. Gardeniers, R. E. Oosterbroek, M. H. J. M. De Croon, J. C. Schouten, A. Van Den Berg, M. C. Elwenspoek, Sensors Actuators, A Phys. 2005, 119, $196-205$.

[38] R. M. Tiggelaar, P. W. H. Loeters, P. Van Male, R. E. Oosterbroek, J. G. E. Gardeniers, M. H. J. M. De Croon, J. C. Schouten, M. C. Elwenspoek, A. Van den Berg, Sensors Actuators, A Phys. 2004, 112, 267-277.

[39] H. F. Arata, Y. Rondelez, H. Noji, H. Fujita, Anal. Chem. 2005, 77, 4810-4814.

[40] M. A. Burns, B. N. Johnson, S. N. Brahmasandra, K. Handique, J. R. Webster, M. Krishnan, T. S. Sammarco, P. M. Man, D. Jones, D. Heldsinger, C. H. Mastrangelo, D. T. Burke, Science (80-. ). 1998, 282, 484-487.

[41] D. Jaque, F. Vetrone, Nanoscale 2012, 4, 4301-4326.

[42] C. D. S. Brites, P. P. Lima, N. J. O. Silva, A. Millán, V. S. Amaral, F. Palacio, L. D. Carlos, Nanoscale 2012, 4, 4799-4829.

[43] T. Li, C. Guo, S. Zhou, C. Duan, M. Yin, J. Am. Ceram. Soc. 2015, 98, 2812-2816.

[44] F. Vetrone, R. Naccache, A. Zamarrón, A. J. De La Fuente, F. Sanz-Rodríguez, L. M. Maestro, E. M. Rodriguez, D. Jaque, J. G. Sole, J. A. Capobianco, ACS Nano 2010, 4, 3254-3258.

[45] R. G. Geitenbeek, P. T. Prins, W. Albrecht, A. Van Blaaderen, B. M. Weckhuysen, A. Meijerink, J. Phys. Chem. C 2017, 121, 3503-3510.

[46] R. G. Geitenbeek, J. C. Vollenbroek, H. M. H. Weijgertze, C. B. M. Tregouet, A.-E. Nieuwelink, C. L. Kennedy, B. M. Weckhhuysen, D. Lohse, A. van Blaaderen, A. van den Berg, M. Odijk, A. Meijerink, Lab Chip 2019, 19, 1236-1246.

[47] I. C. L. Buurmans, Catalyst Particles for Fluid Catalytic Cracking Visualized at the Individual Particle Level By, Utrecht University, 2011. 
Development of a Droplet Microreactor for the High-Throughput Fluorescence-Based Detection of Single Fluid Catalytic Cracking Particle Activity 


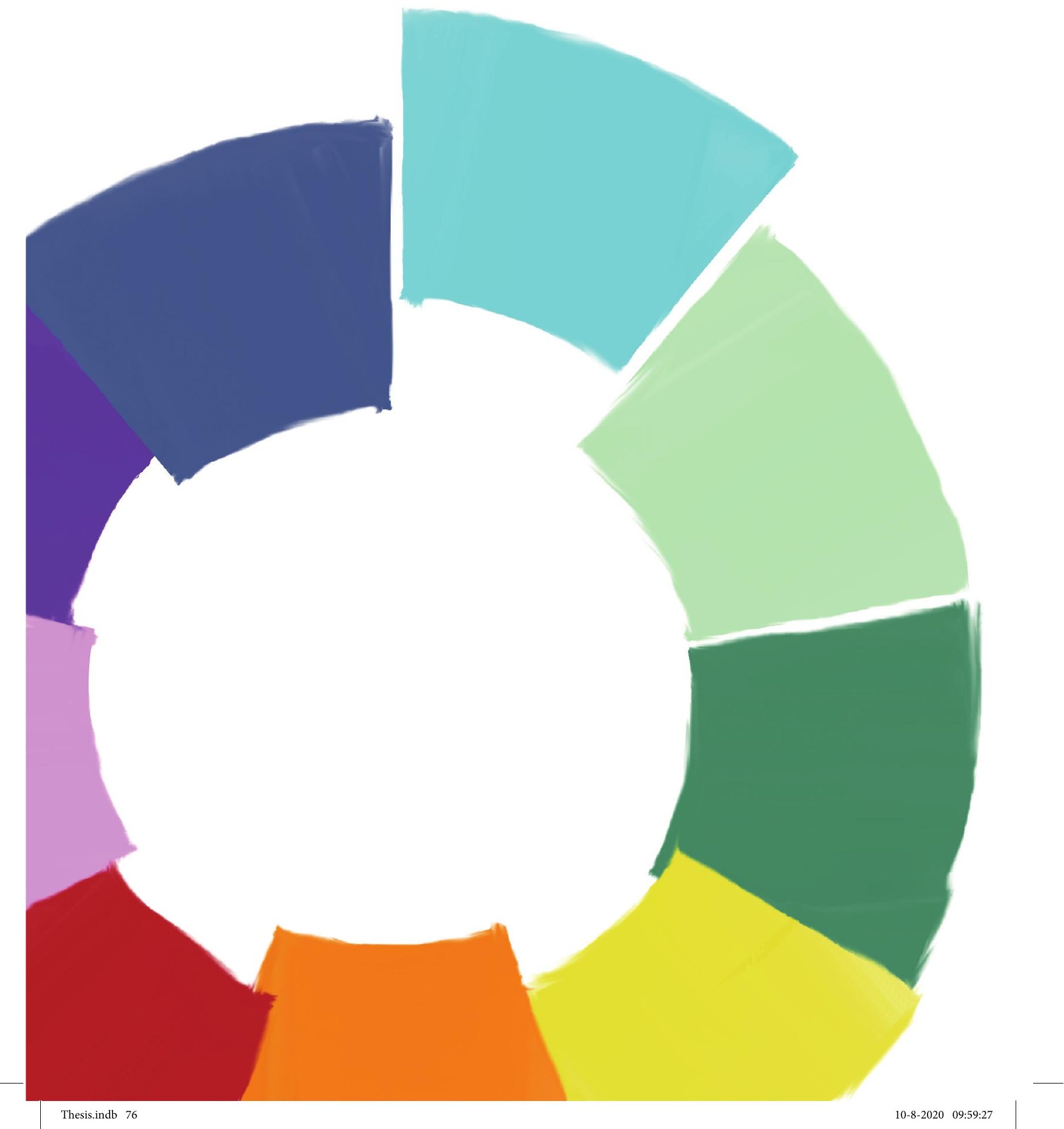




\section{Chapter 5 \\ High-Throughput Activity Screening and Sorting of Fluid Catalytic Cracking Particles with a Droplet Microreactor Equipped with Dielectrophoresis and Fluorescence Detection}

In this Chapter, we describe the development of a droplet microreactor for the high-throughput screening and sorting of Fluid Catalytic Cracking (FCC) Equilibrium catalyst (ECAT) particles based on dielectrophoresis (DEP). ECAT particles stained with 4-fluorostyrene were sorted and analyzed using fluorescence microscopy and micro X-ray fluorescence $(\mu-X R F)$. This leads to a sorting system based on the accessible Bronsted acid sites that the ECAT particles have. The post-sorting characterization confirms that the sorted particles are indeed the most fluorescent and, therefore, most acidic particles. Furthermore, analysis showed that the non-sorted particles contain higher $\mathrm{Ni}$ levels than the sorted particles, indicating that the non-sorted particles are the more aged particles, since $\mathrm{Ni}$ is not present in fresh particles and can only be accumulated by spending time in an industrial reactor.

This work is based on the following manuscript: A.-E. Nieuwelink*, J.C. Vollenbroek*, J.G. Bomer, R.M. Tiggelaar, A. van den Berg, M. Odijk, B.M. Weckhuysen, High-Throughput Activity Screening and Sorting of Fluid Catalytic Cracking Particles with a Droplet Microreactor Equipped with Dielectrophoresis and Fluorescence Detection, in preparation.

* These authors contributed equally 


\subsection{Introduction}

There is a need for an automated system that can analyze, and sort single FCC ECAT particles based on their activity in a high-throughput fashion. The system that is developed in this chapter uses droplet microfluidics ${ }^{[1]}$ for the high-throughput encapsulation of single FCC particles. Dielectrophoresis (DEP) is used for the sorting of the droplets containing the most active particles. For the post-sorting analysis, micro $\mathrm{X}$-ray fluorescence ( $\mu$-XRF) is used to detect the metal content present in the particles.

Two sorting methods that already exist for the analysis of FCC particles are density separation and magnetic sorting. In both methods particles are sorted based on the amount of accumulated metals in the particles. During density separation, the particles are subsequently immersed in solvent mixtures (acetone/di-iodomethane) with different densities. ${ }^{[2]}$ The accumulated metals on the FCC particles result in a higher skeletal density; making it possible to separate the particles in fractions with more or less accumulated metals. With magnetic sorting, particles containing ferromagnetic metals such as, nickel $(\mathrm{Ni})$ and iron $(\mathrm{Fe})$ can be attracted towards a magnet and sorted out ${ }^{[3]}$, as was described in Chapter 2. It was found that accumulated metals can either form large metal clusters (mostly iron or iron oxides) on the outer surface of the particle or are uniformly distributed and form a shell (2-3 $\mu \mathrm{m}$ deep) over the surface of the particles. ${ }^{[3]}$ Particles with large Fe clusters can be sorted out using a magnet. An advantage of density separation and magnetic sorting is the relative ease with which they can be performed and implemented. These techniques can separate a statistically relevant part of the deactivated particles from the active particles.

However, even though there are enough indications that the metal content of an FCC particle is negatively correlated to its catalytic activity ${ }^{[4,5]}$, this does not have to hold for every case. The Fe clusters described in Chapter 2 that were found on ECAT particles were formed locally, leaving the rest of the particle still accessible for reactants. ${ }^{[6]}$ Furthermore, dealumination (destruction of the zeolite domains) is not detectable by both density separation and magnetic sorting.

As discussed in Chapter 4, single ECAT particles can be encapsulated in droplets. Using integrated microheaters and temperature sensors ${ }^{[7-16]}$ the reaction conditions can be rapidly tuned and screened. For further analysis of the most active particles found in this screening, it is necessary to separate them from the rest of the particles. With the current system, they all end up in the same waste container. When these highly active particles can be sorted out, they can be further analyzed to find more details on the cause of their activity and also on the cause of the deactivation of the low-to-moderately active particles. To improve the current system into a system that can sort out the most active particles, the fluorescence intensity is still used. Based on the fluorescence signal obtained from the particle, a fast decision must be made on whether to sort the particle or not. Making an analogy with the world of biology: for the sorting of cells, microfluidic Fluorescence Activated Cell Sorting (FACS) systems exist, which are used in clinical medicine, with a throughput of 12000 cells per second. ${ }^{[17]}$ From there it seems a small step to sort out 
the most active catalyst particles in this system. Active microfluidic droplet sorters exist which use various physical properties such as electric, thermal, acoustic, pneumatic, and magnetic forces to sort out droplets of interest from a continuous stream of droplets. ${ }^{[18]}$ Active sorting is fast, and the droplet response is typically in the order of microseconds to milliseconds. For example, electrical control provides fast feedback, resulting in accurate sorting at high-throughput. ${ }^{[18]}$ A proper electrical sorting is based on either the presence of free charges in the droplet, or a high enough contrast in permittivity and conductivity between the droplet and the medium. The latter requirement is used in a sorting technique called dielectrophoresis (DEP). The idea and concept of using DEP to sort and manipulate cells and particles already exists since the late nineties. ${ }^{[19]}$ In this chapter DEP is used to actively sort out droplets containing the most fluorescent particles from a stream of empty droplets and low-to-moderate fluorescent particles. This technique was chosen because of the easy implementation of electrodes in the microreactor, and the fast and accurate feedback and control that is possible.

Dielectrophoresis (DEP) is a technique that uses non-homogeneous electric fields to attract or repel particles, cells, and droplets suspended in a medium. The idea of dielectrophoresis has been used widely in microfluidics to manipulate a large variety of biological cells and beads. ${ }^{[20-24]}$ For example, size based particle sorting has been achieved ${ }^{[20-22]}$, as well as live-dead sorting of cells ${ }^{[23]}$ and other types of manipulation of cells using DEP. ${ }^{[24]}$ Instead of manipulating cells and beads, it is also possible to manipulate droplets ${ }^{[25-28]}$. Again the sorting of cells that are encapsulated in droplets gives the inspiration to move from cell sorting to catalyst particle sorting. ${ }^{[27,28]}$ A perfect example from literature is the study form Baret et al., where they sort droplets based on a fluorescent signal. This technique shows a lot of potential for fluorescence-based activity sorting of catalyst particles encapsulated in droplets, where a throughput of 2000 droplets per second (with 1 cell in every 50 droplets) was achieved. ${ }^{[27]}$

In all these systems the force generated by the non-homogeneous electric field (DEP force) acting on a cell, particle or droplet, can be described by the following Equation 5.1. ${ }^{[22,25,29]}$

$$
\mathrm{F}_{\mathrm{DEP}}=2 \pi \mathrm{r}^{3} \varepsilon_{\mathrm{m}} \operatorname{Re}\left[\mathrm{F}_{\mathrm{CM}}\right] \mathrm{dE}^{2}{ }_{\mathrm{rms}}
$$

Where $r(m)$ is the radius of the particle, $\varepsilon_{\mathrm{m}}\left(\mathrm{Fm}^{-1}\right)$ is the absolute permittivity of the medium, $\mathrm{E}\left(\mathrm{Vm}^{-1}\right)$ the electric field, and $\mathrm{Re}\left[\mathrm{F}_{\mathrm{CM}}\right]$ is the real part of the Claussius-Mossotti factor $\left(\mathrm{F}_{\mathrm{CM}}\right)$. The Claussius-Mossotti factor determines if the droplet will be attracted (positive DEP or $\mathrm{pDEP}$ ) or repelled (negative $\mathrm{DEP}$ or $\mathrm{nDEP}$ ) from the electric field gradient. The expression for the Claussius-Mossotti factor ${ }^{[22,25,29]}$ is given in Equation 5.2.

$$
\mathrm{F}_{\mathrm{CM}}=\left[\frac{\varepsilon_{\mathrm{d}}^{*}-\varepsilon_{\mathrm{m}}^{*}}{\varepsilon_{\mathrm{d}}^{*}+2 \varepsilon_{\mathrm{m}}^{*}}\right]
$$

Here $\varepsilon_{\mathrm{d}}{ }^{*}\left(\mathrm{Fm}^{-1}\right)$ and $\varepsilon_{\mathrm{m}}{ }^{*}\left(\mathrm{Fm}^{-1}\right)$ are the complex permittivity of the droplet $(\mathrm{d})$ and the 
medium (m). The general expression for $\varepsilon^{*}$ is given in Equation 5.3.

$$
\varepsilon^{*}=\varepsilon_{0} \varepsilon-\frac{j \sigma}{\omega}
$$

Where $\varepsilon_{0}\left(\mathrm{Fm}^{-1}\right)$ is the permittivity of free space, $\varepsilon$ is the dielectric constant of a specific material, $\sigma\left(\mathrm{Sm}^{-1}\right)$ is the conductivity of a specific material and $\omega\left(2 \pi \mathrm{s}^{-1}\right)$ is the angular frequency. When combining equation 3 and equation 2, a formula for the real part of the Claussius-Mossotti factor $\operatorname{Re}\left(\mathrm{F}_{\mathrm{CM}}\right)$ can be found. The equation is given in equation 4 .

$$
\operatorname{Re}\left(F_{C M}\right)=\frac{\omega^{2}\left(\varepsilon_{d}-\varepsilon_{m}\right)\left(\varepsilon_{d}+2 \varepsilon_{m}\right)+\left(\sigma_{d}-\sigma_{m}\right)\left(\sigma_{d}+2 \sigma_{m}\right)}{\omega^{2}\left(\varepsilon_{d}+2 \varepsilon_{m}\right)^{2}+\left(\sigma_{d}+2 \sigma_{m}\right)^{2}}
$$

Where $\varepsilon_{\mathrm{d}}\left(\mathrm{Fm}^{-1}\right)$ and $\varepsilon_{\mathrm{m}}\left(\mathrm{Fm}^{-1}\right)$ and $\sigma_{\mathrm{d}}\left(\mathrm{Sm}^{-1}\right)$ and $\sigma_{\mathrm{m}}\left(\mathrm{Sm}^{-1}\right)$ are the absolute permittivity and the conductivity of the droplet (d) and surrounding medium (m) respectively. The Claussius-Mossotti factor describes the frequency dependent contrast between the permittivity and conductivity of the droplet and the medium. This contrast can be interpreted as the difficulty level to polarize a droplet with respect to the medium. Furthermore, the contrast determines whether a droplet is attracted or repelled from the electric field gradient. For example, when the droplet-phase is easier to polarize (i.e. it has a higher permittivity and conductivity) than the continuous phase, the $\mathrm{F}_{\mathrm{CM}}$ is positive with a maximum of 1 . In this case the DEP force is directed towards the electric field gradient. When the continuous phase is easier to polarize (i.e. higher permittivity and conductivity) compared to the droplet phase, than the $\mathrm{F}_{\mathrm{CM}}$ is negative with a maximum of - 0.5. In this case the DEP force is directed away from the electric field gradient. Finally, the $\mathrm{F}_{\mathrm{CM}}$ also describes the efficiency of the DEP force with a maximum of 1 for $\mathrm{pDEP}$ and maximum of -0.5 for nDEP. The larger the contrast polarizability between the droplet and the continuous phase, the more efficient the applied DEP force is ${ }^{[22,25,29]}$.

In this Chapter, we describe the development of a droplet microreactor equipped with dielectrophoresis (DEP) for the high-throughput screening and sorting of ECAT particles based on their fluorescence after staining with 4-fluorostyrene. After sorting, these particles were analyzed with fluorescence microscopy and micro X-ray fluorescence ( $\mu$-XRF), similar to what has been described in Chapter 3.

\subsection{Experimental Section}

\subsubsection{Microreactor Design}

The design of the glass/glass DEP microreactor, drawn with Clewin software, is shown in Figure 5.1. The microreactor has both a reactor zone and a sorting window and the design will now be discussed moving from inlet to outlet. This design has 'side' inlets (and outlets) which are in line with the flow direction in the microreactor. This design is based on chipholders from Micronit Microtechnologies and different from the silicon/ glass microreactor used in Chapter 4, where the in- and outlets where located at the top, i.e. orthogonal to the flow direction in the microreactor. This is done to prevent particles 
from getting stuck in the $90^{\circ}$ bend upon entering the chip in the previous design. After the inlets there is again a droplet generator to create droplets in which particles are trapped. For the reactor zone, several designs were made. The top design, as shown in Figure 5.1 has broader sections to slow down the lateral velocity of the droplets and increase the residence time. The bottom design, as shown in Figure 5.1, has sloped channels. The sloped channels are meant to prevent particles from getting stuck at the channel wall, when the microreactor is placed in a vertical orientation with the outlets pointing towards the ground, as shown later in Section 5.2.5. In the sorting zone, there are needle-like electrodes embedded, the so-called DEP electrodes. The sharp needle shape is designed to induce a non-homogeneous electric field when a voltage is applied between these needles. After the DEP electrodes, the channel branches into two outlets. One for the actively sorted droplets (sorted outlet) with highly active particles and one for the bulk droplets containing low-to-moderately active particles or no particle at all (non-sorted outlet). The junction of these channels is slightly off-center, and the non-sorted outlet is wider (i.e. has a lower fluidic resistance) than the sorted outlet, to stimulate non-sorted droplets to enter the non-sorted outlet by default. There are small slits that connect the two outlets immediately after the junction to balance possible pressure differences when a droplet or particle enters either of the channels. These slits, also called shunts, have been tested and used in droplet sorting before by Agresti et al. ${ }^{[28]}$ A droplet entering a channel changes the viscosity of the liquid, which changes the fluidic resistance and thus

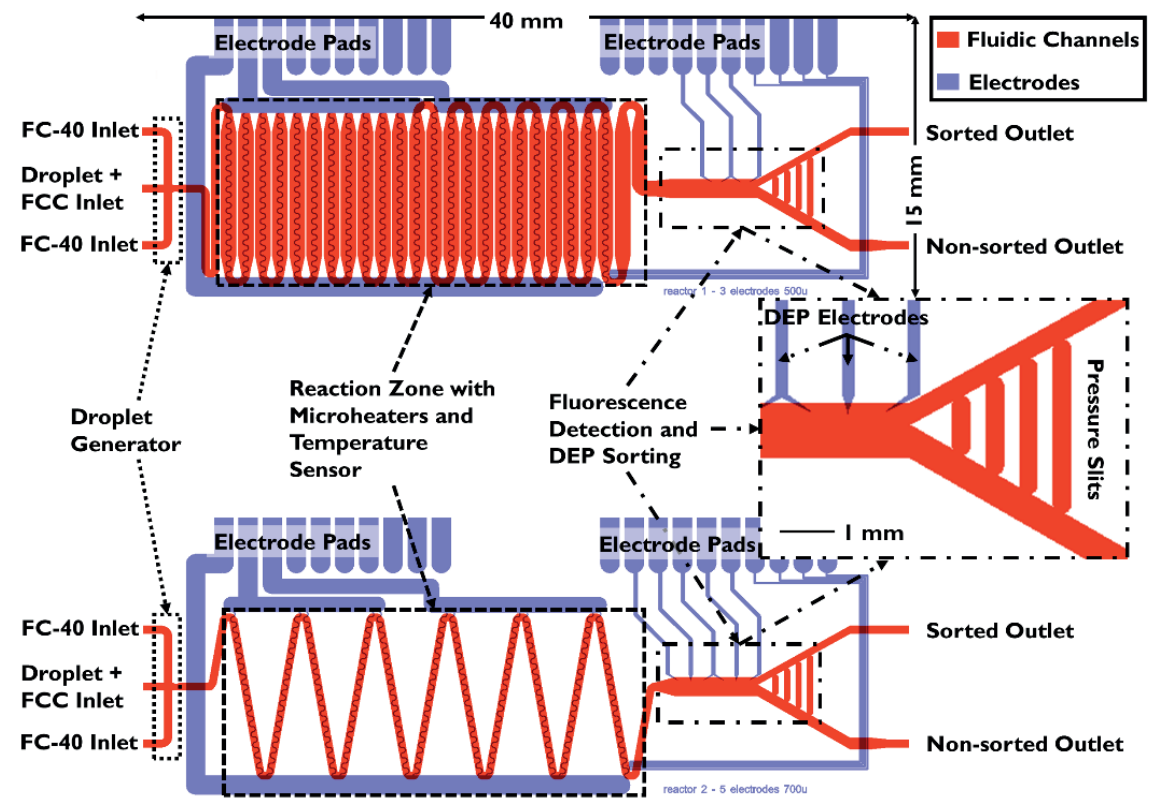

Figure 5.1: Clewin design of the DEP microreactor showing the various features in the fluidic and electronic design layers. Features such as the droplet generator, reaction zone with microheaters and temperature sensor, fluorescence detection zone with DEP electrodes and the sorting junction where the channel splits into a 'sorted' outlet and 'non-sorted' outlet are shown. The inset shows the pressure shunts connected the two outlets and balancing possible pressure differences. The fluidic channels are $400 \mu \mathrm{m} \times 150 \mu \mathrm{m}$ width and height. The sorting sone is $1 \mathrm{~mm}$ wide and $150 \mu \mathrm{m}$ in height. 
the pressure drop. These disturbances can make the junction unstable causing droplets to randomly enter either outlet. By connecting the two outlets with small shunts the pressures between the channels are linked and will keep the junction stable.

\subsubsection{Fabrication}

The microreactors are fabricated in the cleanroom of the MESA+ NanoLab of the University of Twente. Figure 5.2 shows an overview of the executed process steps. The microreactor is a stack of two $500 \mu \mathrm{m}$ thick Mempax glass substrates. Similar to Chapter 4, a three-mask process is used to fabricate the DEP microreactor. The substrate containing the thin-film electrodes is processed in almost the same way as for the silicon/ glass microreactor. Buffered HF (BHF) as a wet etchant is used to etch $200 \mathrm{~nm}$ deep into the top glass substrate, to create the pattern for the Pt structures (i.e. heaters, temperature sensors, and DEP electrodes). This process is directly followed by the deposition of a 10 $\mathrm{nm}$ thick tantalum adhesion layer and a $190 \mathrm{~nm}$ thick platinum layer. All the thin film metallic structures are thus embedded in the glass substrate. Subsequently, the glass substrate containing the $\mathrm{Ta} / \mathrm{Pt}$ structures is covered with a $1.5 \mu \mathrm{m} \mathrm{SiO}$ layer deposited with Plasma Enhanced Chemical Vapour Deposition (PECVD). After deposition, $500 \mathrm{~nm}$ is removed from this layer using Chemical Mechanical Polishing (CMP) to reduce the surface roughness. After polishing a $1 \mu \mathrm{m}$ (PECVD) $\mathrm{SiO}_{2}$ is left that is sufficiently smooth for the fusion bonding process. The $\mathrm{SiO}_{2}$ is then selectively removed from the contact electrodes. The fluidic channels are etched $150 \mu \mathrm{m}$ deep into the bottom glass substrate using hydrofluoric acid (HF) as a wet etchant. Etching glass with HF is an isotropic process resulting in semicircular shaped channels as shown in Figure 5.2. Electronic

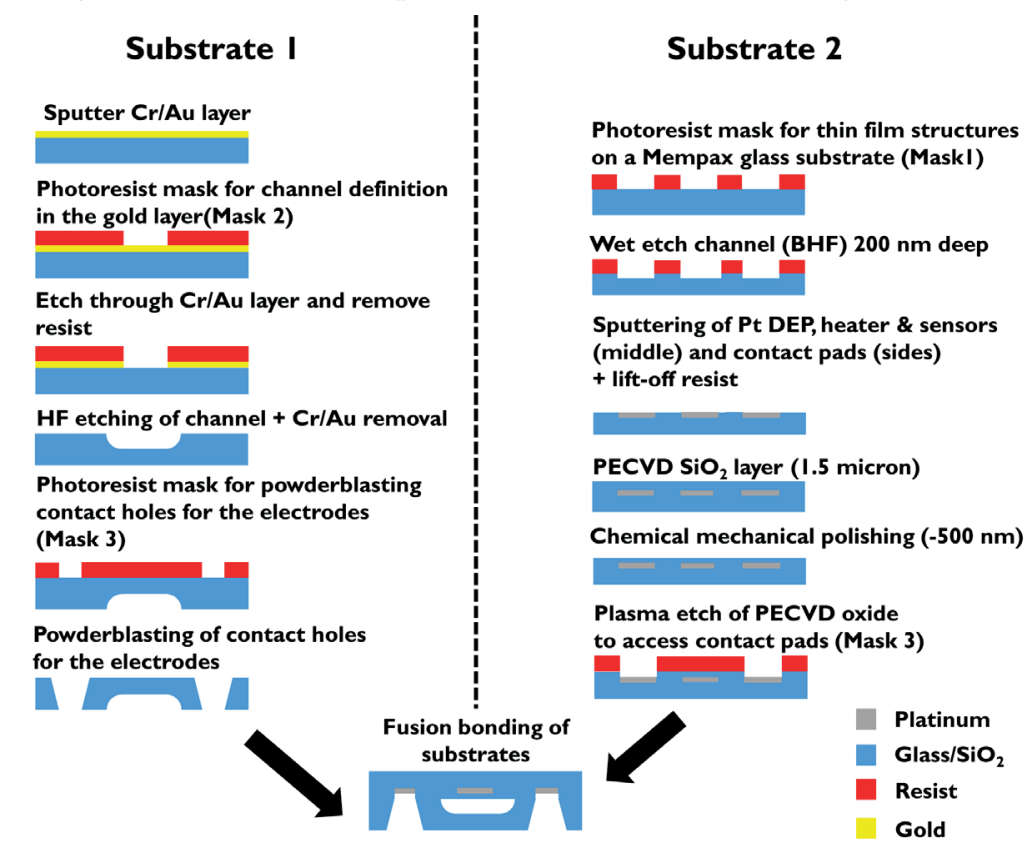

Figure 5.2: Schematic cross-sectional representation of the various steps used to fabricate the DEP microreactor. 
accesses are powder blasted from the backside of the channel substrate. This ensures accessibility of the electrodes embedded in the glass. Finally, the two substrates are bonded together using fusion bonding and individual chips (40x15x1 mm) are created by means of dicing. The fabrication result can be seen in Figure 5.3 where all the fabricated features are indicated.

\subsubsection{Fluidic Setup}

The holder for the microreactor combines a commercial chipholder enabling side-connections (Micronit Microtechnologies, Enschede, The Netherlands) with a homemade chipholder body made of black Delrin, which is designed with Solidworks and realized by milling (Datron NEO, Mühltal, Germany). The holder is shown in Figure 5.3. Small ferrules in the side-connector are used to make a leak-free connection between microreactor and tubing.

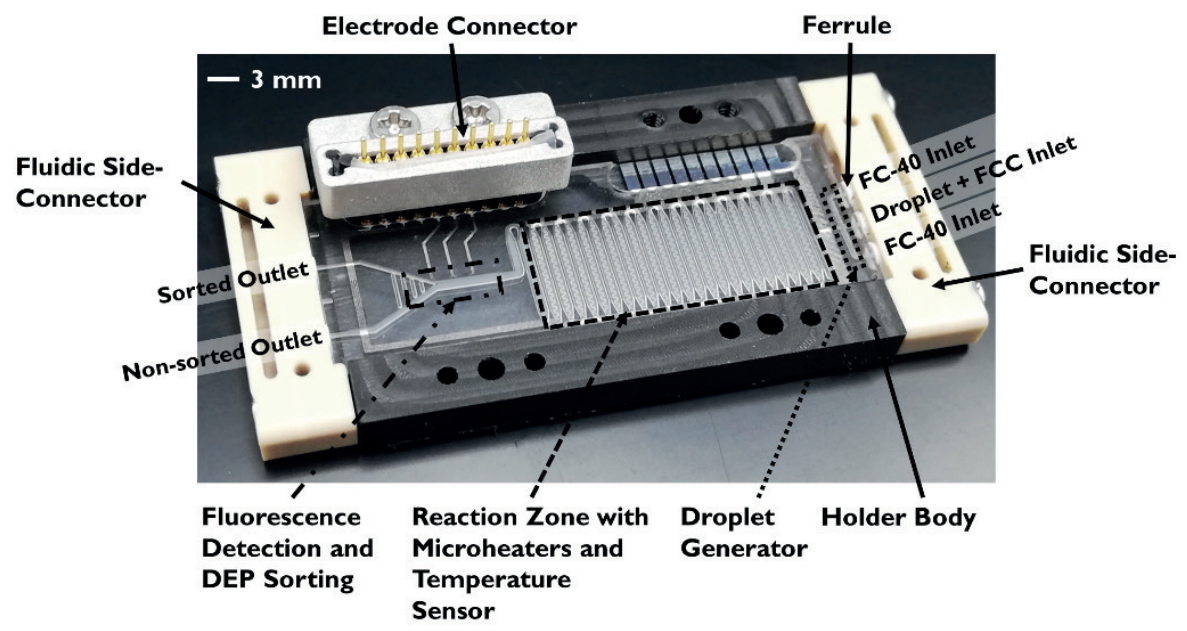

Figure 5.3: Fabricated DEP microreactor including fluidic side-connectors, ferrules, holder body, and electrical connector. The various features on the microreactor, such as the inlets, droplet generator, reaction zone with microheaters and temperature sensors, fluorescence detection and DEP sorting zone, and the outlets are indicated.

A mixture of glycerol and dimethylsulfoxide (DMSO) with a volume ratio of 7:3 (glycerol:DMSO) (both Sigma Aldrich) is used for the droplet phase and for the continuous phase fluorinated FC-40 oil (3M, St Paul, USA) is used. An excess of $\mathrm{NaCl}$ has been added to the droplet phase mixture to increase the conductivity and favor the Clausius-Mossotti factor at DC $(0 \mathrm{~Hz})$ and low frequency voltages. From Eq. 5.4 it can be seen that the conductivity determines the $\mathrm{F}_{\mathrm{CM}}$ for $\mathrm{DC}(0 \mathrm{~Hz})$ and low frequencies. After heating the mixture to $80^{\circ} \mathrm{C}$ for 10 minutes the undissolved $\mathrm{NaCl}$ is centrifuged out with an Allegra X-12R Centrifuge (Beckman Coulter) and the conductivity is measured with a conductivity sensor (Seven Multi, Mettler Toledo, Zaventem, Belgium) and found to be $22 \cdot 10^{-3} \mathrm{Sm}^{-1}$. All parameters for determining the $\mathrm{F}_{\mathrm{CM}}$ are given in Table 5.1. With these parameters and for a DC voltage $(0 \mathrm{~Hz})$ the $\mathrm{F}_{\mathrm{CM}}$ has its maximum value of 1 for pDEP.

A Nemesys syringe pump (Cetoni GmbH, Korbussen, Germany) fitted with glass 
Table 5.1: Physical properties of the used liquids in calculating the Claussius-Mossotti factor.

\begin{tabular}{c||c|c} 
& Conductivity $\left(\mathrm{Sm}^{-1}\right)$ & Relative permittivity \\
\hline \hline Glycerol/DMSO (7:3 mixture) & $2210^{-3}$ & $20-40$ \\
\hline FC-40 & $2.510^{-14}$ & 1.9
\end{tabular}

Hamilton syringes $(5 \mathrm{~mL}$ for the continuous phase and $500 \mu \mathrm{L}$ for the droplet phase) and controlled with Nemesys software is used. As described in Chapter 4, the same precautions (stirring magnet and vibrational motor) are added to the particle containing syringe. Polymer tubing (Tefzel, OD = 1/16”, ID = 0.02”, Idex HS, Overberg, The Netherlands) in combination with fluidic connectors (Inacom Instruments) is used to connect the syringes with the inlet of the microreactor. The same type of tubing is fitted to the outlets of the microreactor. Both outlets have a separate collection reservoir where filter paper (Whatman 1001-070 grade 1 filter paper) is used to collect the particles. Before the creation of glycerol/DMSO-in-oil droplets, the channel walls are made hydrophobic by flushing the microreactor with a solution of $5.55 \mu \mathrm{L}$ tridecafluoro-1,1,2,2tetrahydrooctyltrichlorosilane (FOTS) added to $1.5 \mathrm{~mL}$ FC-40 oil. Prior to experiments the microreactor is flushed for 45 minutes with the FOTS and FC-40 solution and then for 45 minutes with only FC-40. For the experiments, flowrates of 150 to $200 \mu \mathrm{L} / \mathrm{min}$ for the continuous phase and $1.5 \mu \mathrm{L} / \mathrm{min}$ for the dispersed phase were used. These high flowrates for the continuous phase are necessary to prevent possible droplet coalescence in the broadened sorting zone.

\subsubsection{FCC Staining}

Calcined FCC ECAT particles were placed on a microscopy slide and heated to $100{ }^{\circ} \mathrm{C}$ on a hotplate. $10 \mu \mathrm{L}$ 4-methoxystyrene (Sigma Aldrich, 97\%) or 4-fluorostyrene (Sigma Aldrich, 97\%) was added to the microscopy slide, next to the particles. The slide was covered with a glass Petri dish to allow for diffusion of the styrene derivatives into the FCC particles. The heating was stopped after $10 \mathrm{~s}$. Due to a change in fluid properties that would severely complicate DEP sorting and due to the very short residence time of $2 \mathrm{~s}$ of droplets in the heater zone of the microreactor, the staining of FCC particles was performed ex-situ.

\subsubsection{Fluorescence Detection and Sorting Setup}

An in-house constructed setup is used for the optical detection of the fluorescent particles. This setup has been reported in previous work ${ }^{[30]}$, however some components such as filter, mirrors, and excitation sources are changed for this specific application. Figure 5.4 shows a schematic representation of the microreactor and the fluorescent particle detection system. Droplets containing pre-stained particles are flushed through the microreactor. Upon reaching the sorting zone, they pass through a detection spot where the excitation wavelength for the fluorescent probe is focused through a 20x objective (20x/0.4 HCX PL FLUOTAR, Leica). Due to the configuration of the microchannel ( $150 \mu \mathrm{m}$ deep and $1 \mathrm{~mm}$ wide), the droplets pass through the detection spot all with the same distance to the objective, showing them all in focus. The excitation light is produced with a LED (470 nm excitation, M470L3, Thorlabs, Munich, Germany) 


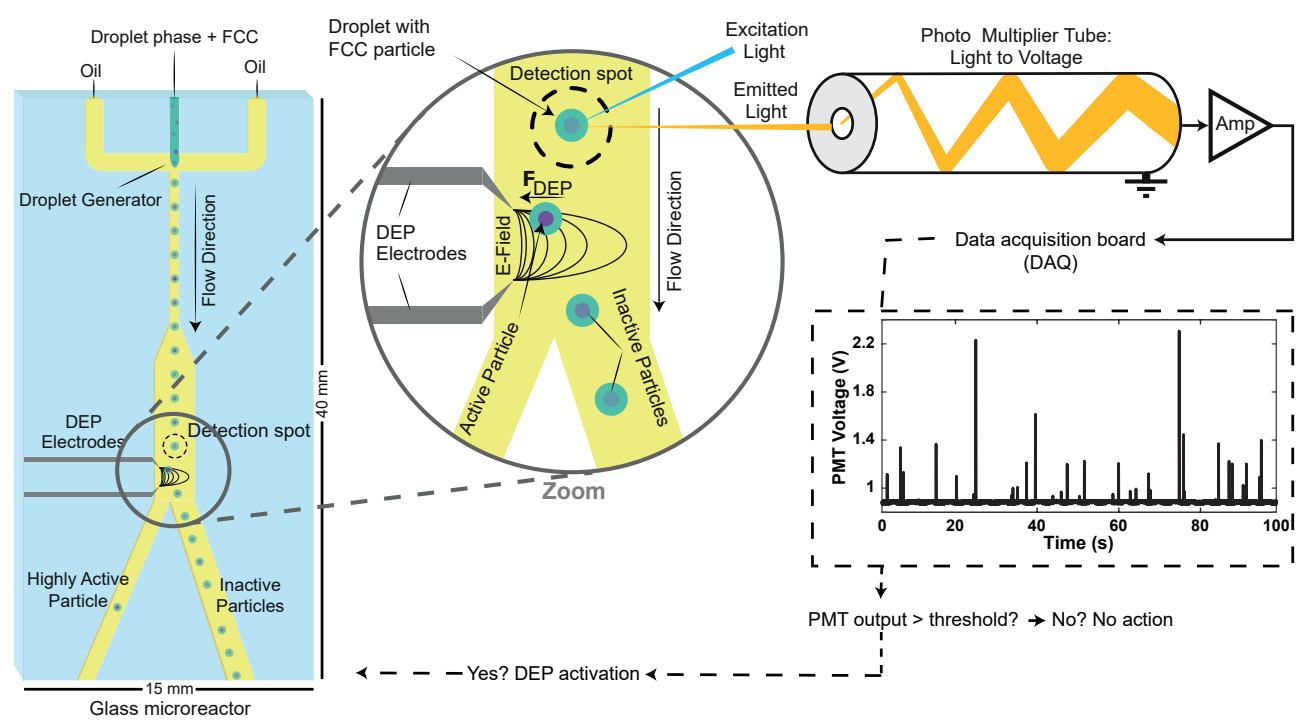

Figure 5.4: Conceptual image of the DEP sorting system showing the microreactor with particles encapsulated in droplets. Droplets pass through a detection spot where the probe molecule present in the particle is excited and subsequently emits light at a different wavelength. The emitted light is detected with an optical setup where a PMT converts the light into a voltage. The voltage is amplified and read by a Data Acquisition System (DAQ). With Labview software, running on the DAQ, the fluorescence peaks are shown in time. A threshold can be set in the software, separating the high peaks from the low peaks. A peak exceeding the threshold activates an electronic switch that controls and activates the DEP electrodes. When activated, the DEP electrodes generate a non-homogeneous electric field that attracts the droplet containing the highly fluorescent particle, towards the electrodes. The manipulated droplet is pulled into a different outlet and the particle is separated from the bulk.

controlled by a LED driver (LEDD1B, Thorlabs). With a beam splitter (BS019, Thorlabs) the excitation light (25\%) is diverted to a silicon photodiode (S121C, Thorlabs) connected to a power detector (PM100USB, Thorlabs) to calculate the optical power used for the excitation of the sample inside the microreactor. Each particle passing through the detection spot will be excited by the LED light (center wavelength: $470 \mathrm{~nm}$ ) and emit light (center wavelength: $610 \mathrm{~nm}$ ) at a different wavelength. The emission signal is picked up by the objective and passed through a dichroic mirror (DMLP550R, Thorlabs) and emission filter (MDF620-52, Thorlabs). Another beam splitter (BS013, Thorlabs) directs $50 \%$ of the light towards a PhotoMultiplier Tube (PMT) (H7422, Hamamatsu, Herrsching am Ammersee, Germany) which converts the optical signal into a voltage, and the remaining $50 \%$ is directed to a CMOS camera (DC1545M, Thorlabs) used for

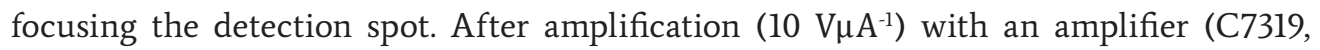
Hamamatsu) the voltage can be read out by a Data Acquisition system (DAQ). A National Instruments (NI) myRio data acquisition bord with Labview software installed is used to measure the output voltage of the PMT. The software measures the voltage from the PMT at a sample rate of $1 \mathrm{kHz}$ during all experiments. As concluded in Chapter 4, highly active particles emit the most fluorescence light, therefore the voltage read by the DAQ is directly correlated with the particle activity. Figure 5.4 shows an example of the voltage peaks over time, visible when fluorescent particles pass the detection spot. A virtual threshold is indicated, above which the software will immediately activate an electronic 


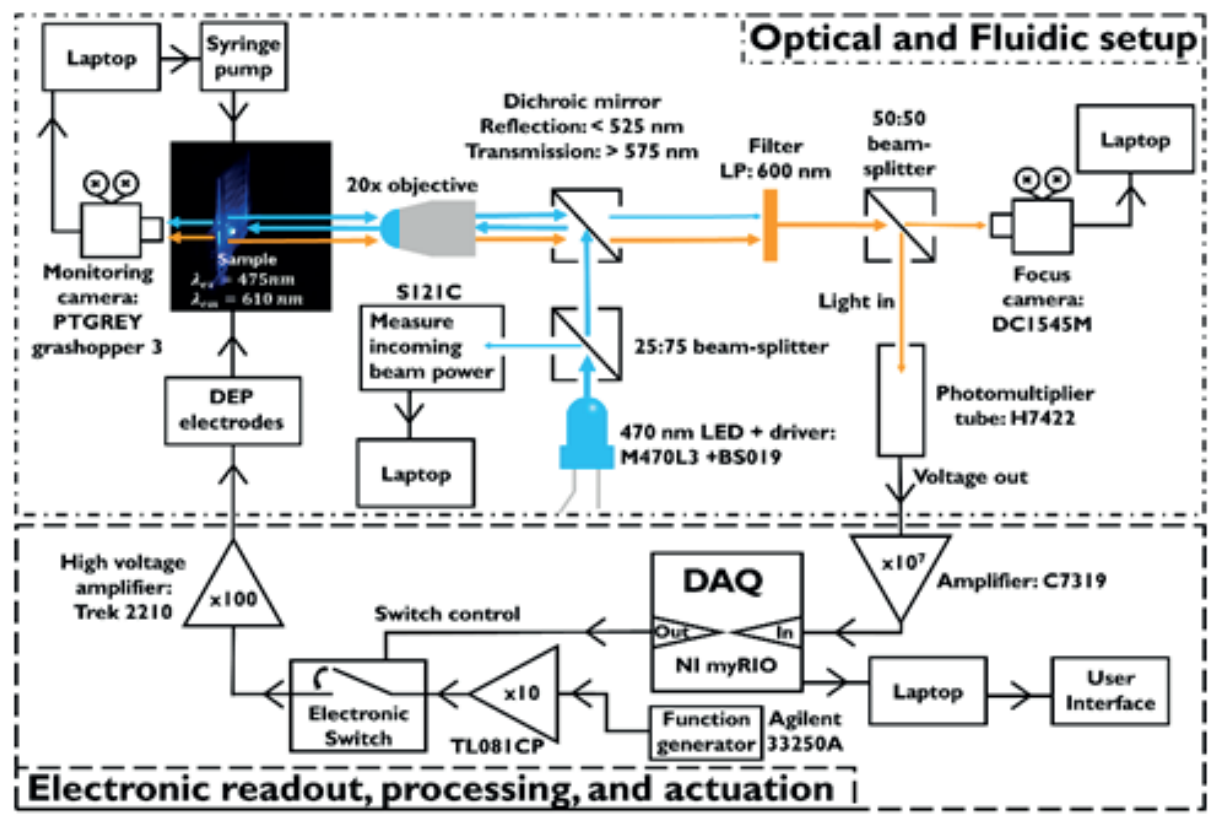

a)

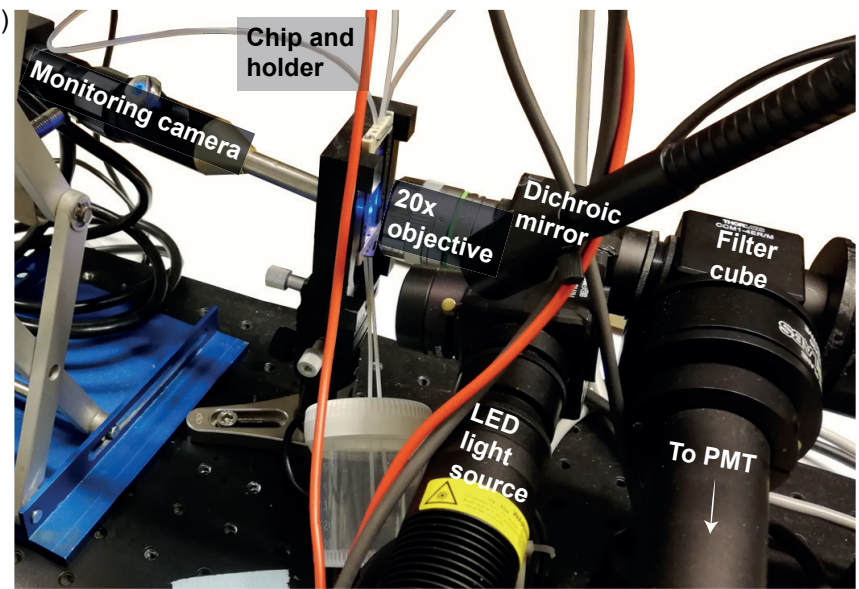

b)

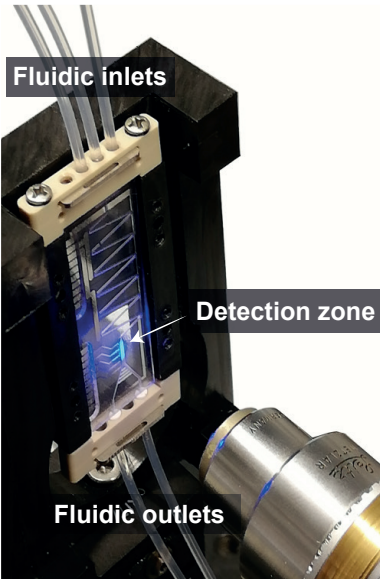

Figure 5.5: Fluorescence detection setup (a) showing the monitoring camera, the objective to focus on the detection zone, and the mirror and filter setup for emitted light detection by a PMT and (b) the microreactor in its holder showing the detection zone and the fluidic in- and outlets. At the bottom, the detailed schematic of the optical and fluidic setup and the electronic processing and activation is shown.

switch, which will 'close' (forming an electrical connection) for a specific amount of time that can be set in the software. If the voltage from the PMT is lower than the threshold, the electronic switch is not activated remains 'open' (forming no electrical connection). The electronic switch is made in house and is placed in between a function generator (Agilent 33250A, Santa Clara, USA) and a high voltage amplifier (Trek model 2210, Trek Inc., Lockport (NY), USA). When the switch is activated, the two systems are connected and the voltage coming from the function generator is amplified with a factor 100. The output voltage of the high voltage amplifier is applied between the DEP electrodes. Initial 
tests showed that the sorting of a droplet using DEP works best when a DC voltage (0 $\mathrm{Hz}$ ) of $600 \mathrm{~V}$ is applied for $95 \mathrm{~ms}$ between the DEP electrodes. Movies of droplets passing the detection zone are recorded with a PTGREY grasshopper 3 camera (FLIR Integrated Imaging Solutions GmbH, Ludwigsburg, Germany) as shown in Figure 5.5, at $90 \mathrm{fps}$ with a shutter time of 7.075 ms. Furthermore, Figure 5.5b shows the microreactor in vertical orientation with the sloped channels as discussed in Section 5.2.1.

\subsubsection{Post-Sorting Analysis of ECAT Particles}

FCC ECAT particles coming out of the microreactor that were collected on the filter papers, are transferred to a microscopy slide with double sided tape. The particles were consecutively analyzed with micro X-ray fluorescence spectroscopy ( $\mu$-XRF), optical microscopy and fluorescence microscopy. For $\mu-\mathrm{XRF}$, the microscopy slide was mounted in an Orbis PC SDD with a Rh-tube as X-ray source $(30 \mathrm{kV}$ and $200 \mathrm{nA})$. The surface was scanned with a spot size of $30 \mu \mathrm{m}$ and a step size of $15 \mu \mathrm{m}$ (300 ms integration time) to obtain an XRF map with the highest intensity per pixel for every element. For optical images, a Zeiss Axio Zoom.V16 microscope using top illumination was used. The microscope was equipped with a PlanNeoFluar Z 1x zoom objective and an Axiocam 105 color camera. The fluorescence intensity was measured with a Nikon Eclipse 90i confocal fluorescence microscope with a Nikon-Eclipse AlR scan head (10x objective) equipped with a $488 \mathrm{~nm}$ argon ion liquid state Melles Griot laser (40 $\mathrm{mW}$ ). Data of all three techniques are combined and correlations are made for all particles. The overlayed images were segmented in a similar way as described in Chapter 3, to obtain information per single particle. The Matlab segmentation is shown in Figure 5.7. It can be seen that a particle can be divided in multiple domains during the segmentation. All these domains will be shown in the Results and Discussion, section 5.3.3. Most importantly, the segmentation allows for
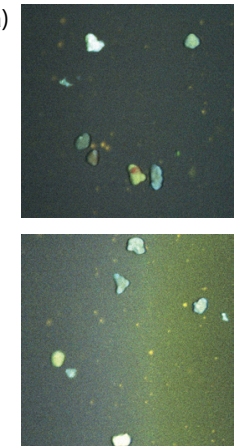

Threshold $=$ 0.1
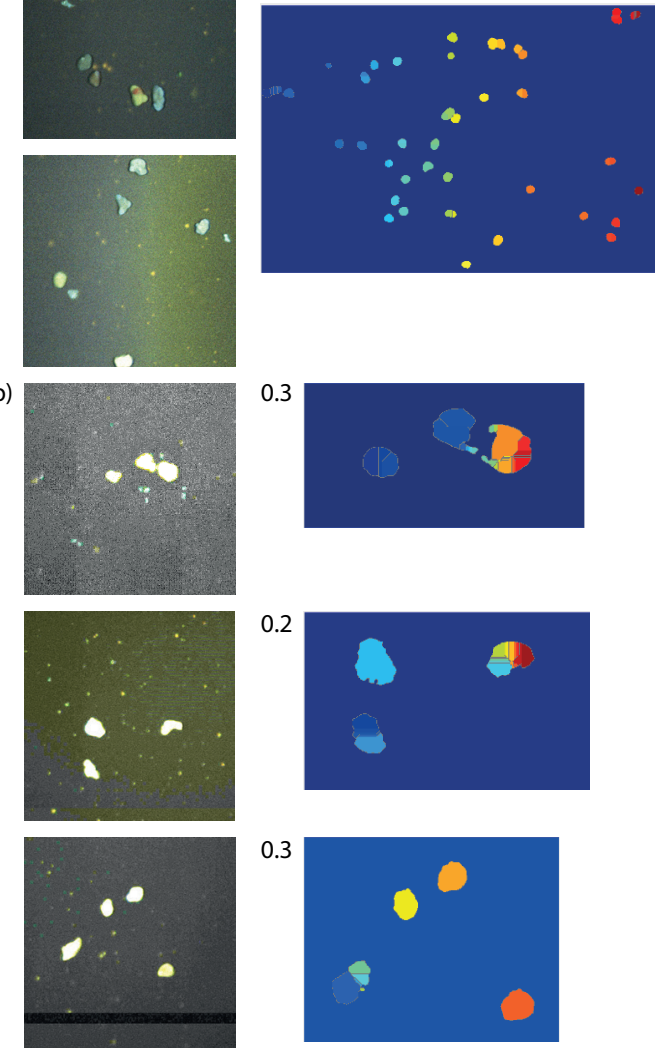

Figure 5.6: The enhanced fluorescence images of the non-sorted (a) and sorted (b) particles and the corresponding Matlab segmentations, show the number of domains counted during the analysis. 


\subsection{Results and Discussion}

After developing the droplet microreactor with implemented DEP sorting, the system first has to be 'trained' in order to recognize a correct sorting threshold. This was done by measuring the fluorescence of a batch of FCC ECAT particles stained with 4-methoxystyrene, similar to the ones used in Chapter 4. However, sorting is more effective using a more selective reactant, namely 4-fluorostyrene. After finding the ideal threshold for 4-fluorostyrene stained FCC particles, the actual sorting was done and all recovered particles were analyzed with fluorescence microscopy to verify their fluorescence. Furthermore, $\mu$-XRF measurements were performed to correlate the FCC particles' fluorescence with their metal content.

\subsubsection{Fluorescent Particle Detection}

Fluorescent FCC particles stained with 4-methoxystyrene, encapsulated in droplets, are flushed through the microreactor, while the voltage from the photomultiplier tube is monitored with the NI myRIO. Figure 5.7 shows the voltage over time, with various voltage peaks throughout the graph. Each peak corresponds to a passing particle. Movies are recorded simultaneously and the frames containing a particle are matched with the recorded voltage signal. There is a large variation in the height of the voltage peaks, corresponding to the large difference in fluorescent product produced by the particles. In total 401 particles stained with 4-methoxystyrene are detected during this experiment, resulting in the histogram seen on the right-hand side in Figure 5.7. This distribution of fluorescence intensity (shown as induced voltage) is reminiscent of the fluorescence intensity histogram from Chapter 4. The same distribution between low-to-moderate fluorescence intensity on the left-hand side of the histogram and the high fluorescence intensity on the right-hand side of the histogram is observed. Thus, the droplet-based microreactor platform can indeed be used for in-situ detection of fluorescent light from particles encapsulated in droplets.
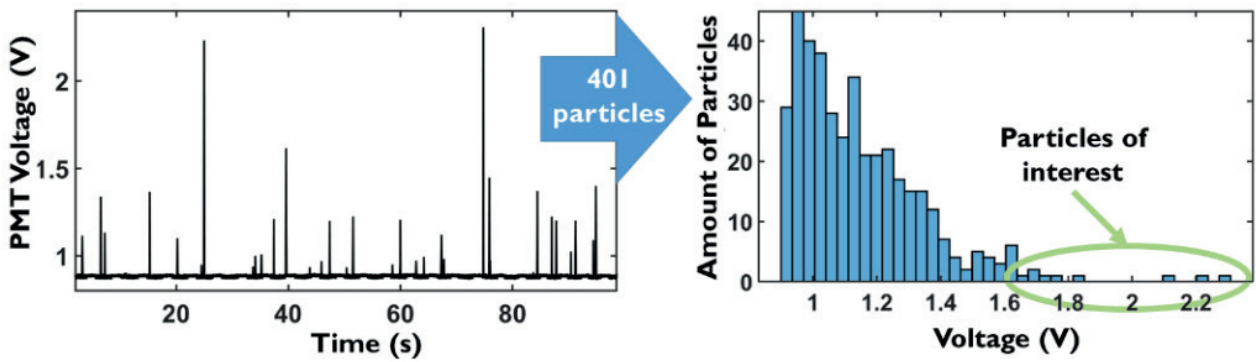

Figure 5.7: On the left-hand side showing the PMT output voltage over time where each peak corresponds to a fluorescent particle passing the detection zone. On the right-hand side a histogram showing the peak voltage distribution for 401 FCC ECAT particles stained with 4-methoxystyrene.

For 4-fluorostyrene the distribution is more binary, because 4-fluorostyrene only oligomerizes on the strongest Brønsted acid sites, meaning that only the most active particles show a strong fluorescence signal. Figure 5.8 shows the histogram for 33 FCC particles passing the detector that are stained with 4-fluorostyrene. Here it is shown that there is no mid-section of moderately active particles, as in Figure 5.7. FCC particles 


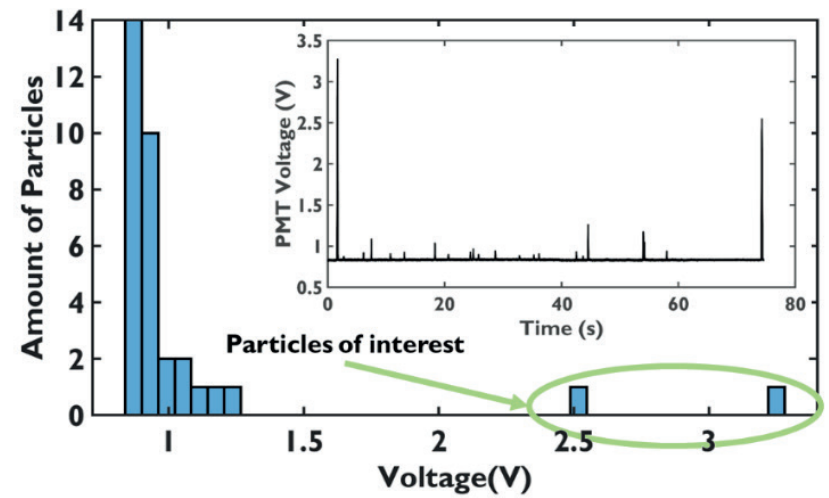

Figure 5.8: Histogram showing the peak voltage distribution for 33 FCC ECAT particles stained with 4-fluorostyrene. The inset shows the PMT output voltage over time, where each peak corresponds to a fluorescent particle.

either give a strong fluorescence signal, or barely any signal at all. The histograms in Figures 7 and 8 are used as training sets to determine a threshold for the sorting that is discussed in the next section. An average rate of $\sim 23$ droplets/s is achieved with a total flowrate of $\sim 182 \mu \mathrm{L} / \mathrm{min}$. The particle influx is not constant, on average every $4 \mathrm{~s}$ a particle passes the detection zone, resulting in a fill rate (i.e. the percentage of droplet containing a particle) of $\sim 1 \%$.

\subsubsection{Fluorescent Particle Sorting}

The next step is to set a threshold in the software and activate the DEP electrodes to sort out the FCC particles that induce the highest fluorescence signal. The decision for activating the DEP electrodes has to be made in 100-200 ms (for a total flowrate of $180 \mu \mathrm{L} / \mathrm{min}$ ) as droplets flow through the detection zone with an estimated velocity of $20 \mathrm{~mm} / \mathrm{s}$. Thresholds are ranging from $1.1 \mathrm{~V}$ to $1.5 \mathrm{~V}$ and various movies are recorded to check whether particles exceeding the set threshold are indeed sorted out. Furthermore, particles below the threshold should follow the path of the empty droplets and are not actively sorted. Table 5.2 shows the sorting percentage at two different thresholds for 4-methoxystyrene.

Figure 5.9a shows the microreactor with the detection and sorting zone highlighted. In Figure 5.9b and c both the situations of a particle exceeding the threshold and a particle below the threshold are shown. A threshold of $1.2 \mathrm{~V}$ is used and Figure 5.9b shows multiple frames of the same FCC particle in a droplet passing through the detection and

Table 5.2: The sorting percentage of successfully sorted FCC particles stained with 4-methoxystyrene for two different thresholds.

\begin{tabular}{c||c|c|c}
$\begin{array}{c}\text { Threshold } \\
\text { voltage (V) }\end{array}$ & $\begin{array}{c}\text { Successfully sorted } \\
\text { particles }\end{array}$ & $\begin{array}{c}\text { Total number } \\
\text { of particles }\end{array}$ & $\begin{array}{c}\text { \% sorted from the } \\
\text { total amount }\end{array}$ \\
\hline \hline 1.2 & $36 / 37(97.3 \%)$ & 74 & $48.6 \%$ \\
\hline 1.5 & $7 / 7(100 \%)$ & 51 & $13.7 \%$ \\
\hline Total & $43 / 44(97.7 \%)$ & 125 & -
\end{tabular}




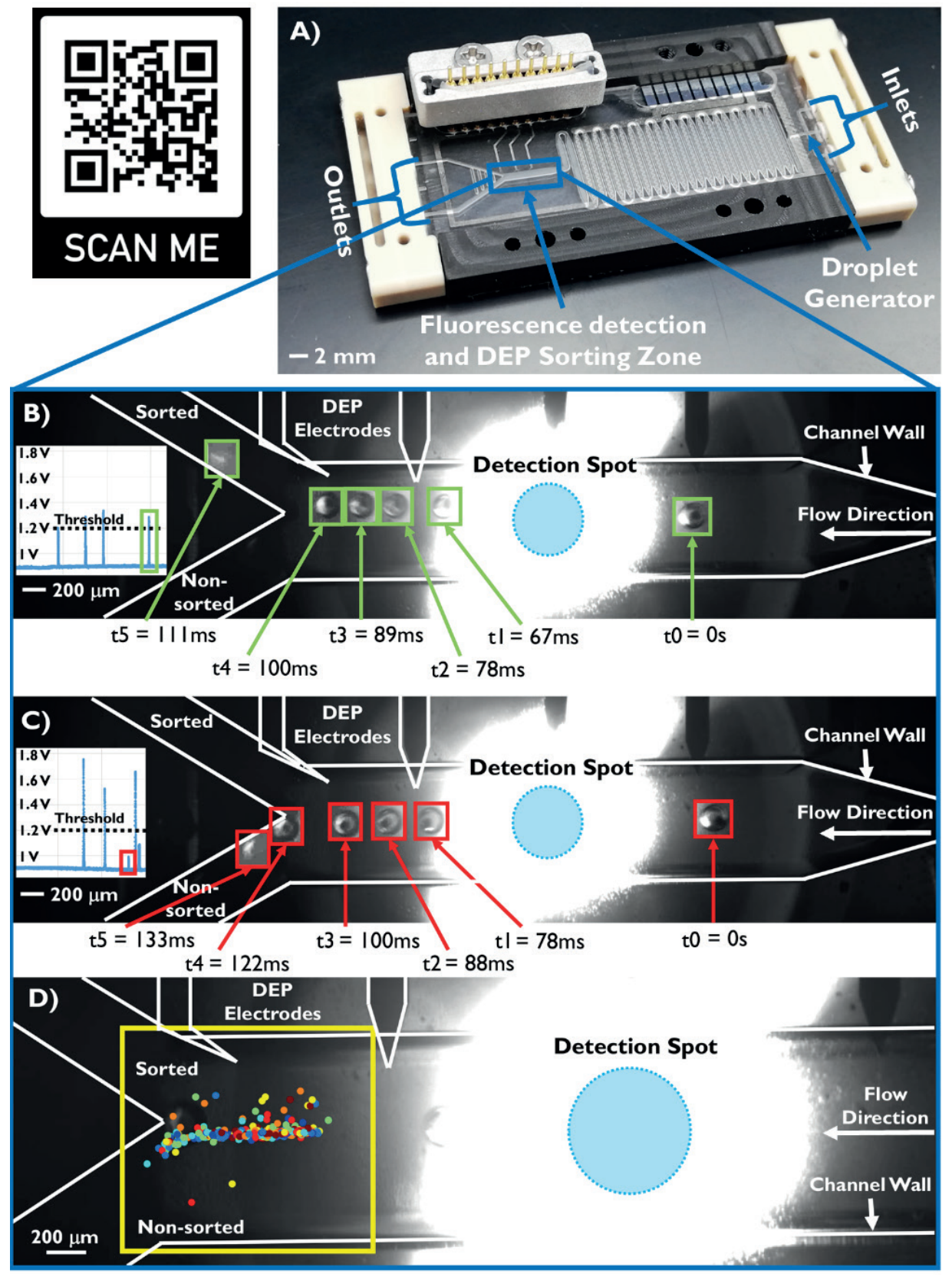

Figure 5.9: a) Image showing the fabricated DEP microreactor and holder with its various features. b) A zoom-in of the fluorescence detection and DEP sorting zone showing multiple frames of a droplet containing a fluorescent particle that moves through the detection spot and exceeds the sorting threshold (1.2 V). c) shows a different droplet containing a particle not exceeding the threshold $(1.2 \mathrm{~V}) \mathrm{d}$ ) the position of every droplet passing the yellow analysis window. The position is determined with DMV software [31]. Contrast and brightness of droplets in the green and red squares in B) and C) are enhanced with Adobe Photoshop for visibility purposes only. The time between the frames is indicated, where t0 is taken as the reference point for each droplet before it reached the detection spot. The flow direction for all images is from right to left. 
sorting zone. It is shown that the droplet starts in the middle of the channel and when the droplet reaches the detection spot (and the threshold is exceeded) the DEP electrodes are activated ( $600 \mathrm{~V}$ for $95 \mathrm{~ms}$ ); pulling the droplet towards the upper 'sorted' channel. Figure 5.9c shows a different FCC particle in a droplet that does not exceed the threshold. This droplet is not pulled into the 'sorted' channel and moves into the lower 'non-sorted' channel. Finally, Figure 5.9d shows the position in the channel for all droplets (empty or with particle) that are passing the yellow analysis area. For this analysis, droplet morphology and velocimetry (DMV) software, developed by Basu et al. ${ }^{[31]}$, is used. The colored dots in the yellow square, in Figure 5.9d, show that the majority of the droplets go into the 'non-sorted' outlet, and that the 'sorted' droplets have a position higher up in the channel.

\subsubsection{Sorted Particle Analysis}

FCC particles stained with 4-fluorostyrene that were in-situ sorted are ex-situ analyzed with fluorescence microscopy and micro X-ray fluorescence ( $\mu$-XRF) spectroscopy, to determine the particles' fluorescence intensity and metal content, respectively. Particles with 4-methoxystyrene are only sorted and in-situ analyzed. Thus, only the 4-fluorostyrene stained FCC particles are ex-situ analyzed in-depth. Only the 4-fluorostyrene stained FCC particles are analyzed in-depth because its oligomerization is more selective for the highly acidic sites, leading to a sorting of only the most active particles. The batch of sorted FCC particles that was used for post-analysis with $\mu$-XRF was sorted with a threshold of $1.1 \mathrm{~V}$. Although, these are not the highest peaks observed, this threshold was chosen to have a higher number of particles for analysis in the sorted collection container.

During the analysis, overlays are made of fluorescence microcopy images and $\mu$-XRF maps, which allows an individual analysis of all FCC particles. Ten sorted particles could be collected and analyzed versus 31 non-sorted particles. The correlation analysis is based on a segmentation of the images and maps after removing the background. This segmentation, however, can cause single particles to be detected as multiple domains. Figure 5.10c shows that FCC particles that came from the sorted collection container (Figure 5.10b) have a much higher fluorescence intensity than the FCC particles in the non-sorted container (Figure 5.10a). For visibility purposes, images with an enhanced brightness and contrast are shown in Figure 5.6. It is observed that most of the nonsorted particles do not show any fluorescence, whereas the sorted particles do clearly exhibit fluorescence, even without adjusting the brightness and contrast. This lack of fluorescence could be due to severe deactivation. This deactivation can be caused by the accumulation of metals such as $\mathrm{Ni}, \mathrm{Fe}, \mathrm{Ca}$ and $\mathrm{V}$ or by dealumination of the zeolite domains. $\mathrm{Ni}$ is one of the metals that is not present in the fresh catalyst particles and can, therefore, be used as a marker for the catalytic age of a particle. It is expected that heavily deactivated FCC particles, have a longer residence time in the reactor unit and thus a higher Ni content. Figure 5.10d shows the metal contents of the sorted and the non-sorted particles. Indeed, particles from the 'sorted' outlet of the DEP microreactor, show lower $\mathrm{Ni}$ and Fe levels compared to the non-fluorescent particles from the 'non- 

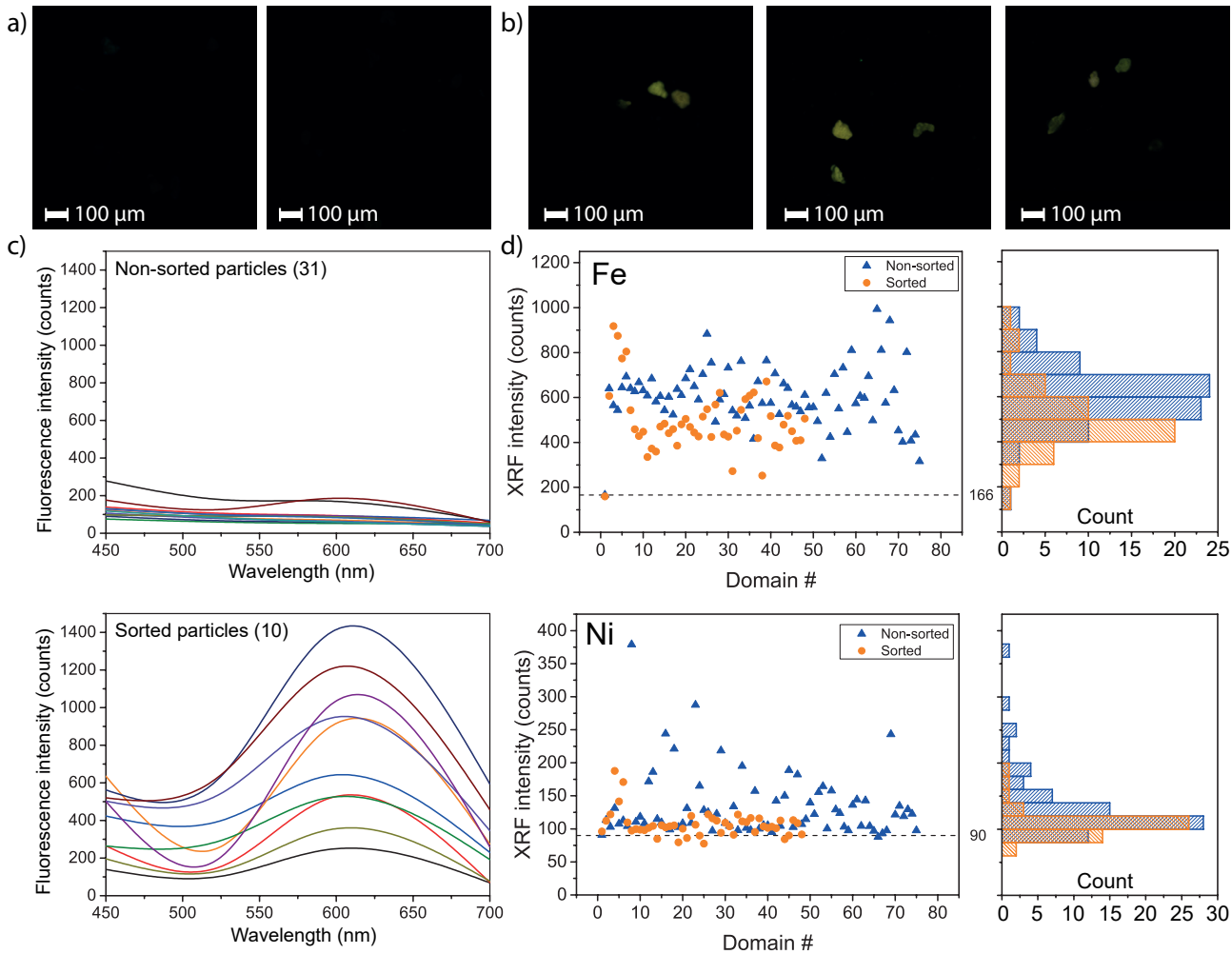

Figure 5.10: Figure 5.10: Fluorescence microscopy images of (a) 'non-sorted' FCC particles and (b) 'sorted' FCC particles, both showing the initial unadjusted image, and (c) their corresponding fluorescence spectra. Enhanced images can be found in Figure 5.6. $\mu$-XRF analysis (d) of the Ni and Fe content in 'sorted' (orange dots) and 'non-sorted' (blue triangles) FCC particles. The first datapoint in both graphs is the background value.

sorted' outlet. It is therefore concluded that the FCC particles sorted via this method, can be considered the least deactivated particles, demonstrating that the most active catalysts can be sorted out using this microreactor for fluorescence activated catalyst sorting. With further analysis of outstanding FCC particles, or sorting at different thresholds, the advantage of using this single particle sorting technique can be exploited further.

\subsection{Conclusions}

In this Chapter we have demonstrated the working principles of a microreactor for the high-throughput, fluorescence activated, catalyst sorting of ECAT particles using dielectrophoresis. Particles encapsulated in droplets and stained with two different fluorescent probe molecules (4-methoxystyrene and 4-fluorostyrene) can be detected and sorted in-situ using an optical setup with a photomultiplier tube for fast feedback on the sorting system. By applying dielectrophoresis, with an electric pulse of 600V DC for $95 \mathrm{~ms}$, the droplets containing highly active particles can be manipulated into a 'sorted' outlet that leads to a collection container, containing only the most active particles. The less active particles with a low fluorescence signal flow unmanipulated into the "nonsorted' outlet. In total 10 sorted particles stained with 4-fluorostyrene are retrieved from 
the 'sorted' collection container. Analysis with fluorescence microscopy confirm that indeed the most fluorescent particles are sorted out and with micro X-ray fluorescence spectroscopy it is shown that these particles have a lower amount of metal (i.e., $\mathrm{Ni}, \mathrm{Fe}, \mathrm{Ca}$, and V) accumulated in them with respect to the unsorted FCC particles. Especially the difference in Ni level between the sorted and non-sorted FCC particles indicates that the most active particles have been sorted out. Furthermore, these are probably the particles that have spent the least amount of time in the industrial reactor as fresh FCC catalyst does not contain any $\mathrm{Ni}$.

The demonstrated fluorescence activated catalyst sorting with a microreactor allows to sort and investigate other catalysts in the future. Screening of freshly synthesized catalyst particles can already contribute valuable information on synthesis parameters. By changing the threshold of this system to only sort out the particles showing the absolute highest fluorescence peaks it could also become possible to sort, for example, zeolite ZSM-5 from zeolite-Y, since ZSM-5 has stronger acid sites than zeolite Y, making the staining with 4-fluorostyrene possibly selective for the zeolite type.

\subsection{Acknowledgments}

Loes Segerink (University of Twente) is acknowledged for help with the statistical validation of the $\mu$-XRF results.

\subsection{References}

[1] X. Casadevall, Chem. Commun. 2011, 47, 1936-1942.

[2] G. R. Dyrkacz, L. Ruscic, C. L. Marshall, W. Reagan, Energy \& Fuels 2000, 71, 849-854.

[3] M. Solsona, A.-E. Nieuwelink, F. Meirer, L. Abelmann, M. Odijk, W. Olthuis, B. M. Weckhuysen, A. van den Berg, Angew. Chem. Int. Ed. 2018, 57, 10589-10594.

[4] E. T. C. Vogt, B. M. Weckhuysen, Chem. Soc. Rev. 2015, 44, 7342-7370.

[5] F. Meirer, S. Kalirai, D. Morris, S. Soparawalla, Y. Liu, G. Mesu, J. C. Andrews, B. M. Weckhuysen, Sci. Adv. 2015, 1, e1400199.

[6] M. Solsona, Single Catalyst Particle Diagnostics: Using Magnetic and Electric Fields., University of Twente, 2019.

[7] R. M. Tiggelaar, R. G. P. Sanders, A. W. Groenland, J. G. E. Gardeniers, Sensors Actuators, A Phys. 2009, 152, 39-47.

[8] W.-Y. Chang, Y.-S. Hsihe, Microelectron. Eng. 2016, 149, 25-30.

[9] V. Miralles, A. Huerre, F. Malloggi, M.-C. Jullien, Diagnostics 2013, 3, 33-67.

[10] J. L. Lin, M. H. Wu, C. Y. Kuo, K. Da Lee, Y. L. Shen, Biomed. Microdevices 2010, 12, 389-398.

[11] C. Fang, D. Lee, B. Stober, G. G. Fuller, A. Q. Shen, RSC Adv. 2015, 5, 85620-85629.

[12] T. Pennell, T. Suchyna, J. Wang, J. Heo, J. D. Felske, F. Sachs, S. Z. Hua, Anal. Chem. 2008, 80, 2447-2451.

[13] A. I. K. Lao, T. M. H. Lee, I. M. Hsing, N. Y. Ip, Sensors Actuators, A Phys. 2000, 84, 11-17.

[14] N. Crews, C. Wittwer, R. Palais, B. Gale, Lab Chip 2008, 8, 919-924.

[15] R. Zhong, X. Pan, L. Jiang, Z. Dai, J. Qin, B. Lin, Electrophoresis 2009, 30, 1297-1305.

[16] R. Phatthanakun, P. Deekla, W. Pummara, C. Sriphung, C. Pantong, N. Chomnawang, ECTI-CON, Annu. Int. Conf. Electr. Eng. Comput. Telecommun. Inf. Technol. Assoc. 2011, 14-17.

[17] A. Wolff, I. R. Perch-Nielsen, U. D. Larsen, P. Friis, G. Goranovic, C. R. Poulsen, J. P. Kutter, P. Telleman, Lab Chip 2003, 3, 22-27.

[18] H.-D. Xi, H. Zheng, W. Guo, A. M. Ganan-Calvo, Y. Ai, C.-W. Tsao, J. Zhou, W. Li, Y. Huang, N.-T. Nguyen, S. H. Tan, Lab Chip 2017, 17, 751-771.

[19] S. Fiedler, S. G. Shirley, T. Schnelle, G. Fuhr, Anal. Chem. 1998, 70, 1909-1915.

[20] N. Lewpiriyawong, C. Yang, Y. C. Lam, Electrophoresis 2010, 31, 2622-2631.

[21] P. R. C. Gascoyne, J. Vykoukal, Electrophoresis 2002, 23, 1973-1983.

[22] J. G. Kralj, M. T. W. Lis, M. A. Schmidt, K. F. Jensen, Anal. Chem. 2006, 78, 5019-5025. 
[23] D. Lee, D. Kim, Y. Kim, K. hyun Park, E. J. Oh, Y. Kim, B. Kim, J. Lab. Autom. 2014, 19, 60-74.

[24] J. Yao, G. Zhu, T. Zhao, M. Takei, Electrophoresis 2019, 40, 1166-1177.

[25] K. Ahn, C. Kerbage, T. P. Hunt, R. M. Westervelt, D. R. Link, D. A. Weitz, Appl. Phys. Lett. 2006, 88, 1-3.

[26] A. M. Pit, R. de Ruiter, A. Kumar, D. Wijnperlé, M. H. G. Duits, F. Mugele, Biomicrofluidics 2015, 9, 044116. [27] J. C. Baret, O. J. Miller, V. Taly, M. Ryckelynck, A. El-Harrak, L. Frenz, C. Rick, M. L. Samuels, J. B. Hutchison, J. J. Agresti, D. R. Link, D. A. Weitz, A. D. Griffiths, Lab Chip 2009, 9, 1850-1858.

[28] J. J. Agresti, E. Antipov, A. R. Abate, K. Ahn, A. C. Rowat, J. C. Baret, M. Marquez, A. M. Klibanov, A. D. Griffiths, D. A. Weitz, Proc. Natl. Acad. Sci. U. S. A. 2010, 107, 6560.

[29] S. L. Tsai, J. L. Hong, M. K. Chen, L. S. Jang, Electrophoresis 2011, 32, 1337-1347.

[30] F. T. G. Van Den Brink, T. Phisonkunkasem, A. Asthana, J. G. Bomer, A. M. J. M. Van Den Maagdenberg, E. A. Tolner, M. Odijk, Lab Chip 2019, 19, 1332-1343.

[31] A. S. Basu, Lab Chip 2013, 13, 1892-1901. 
High-Throughput Activity Screening and Sorting of Fluid Catalytic Cracking Particles with a Droplet Microreactor Equipped with Dielectrophoresis and Fluorescence Detection 


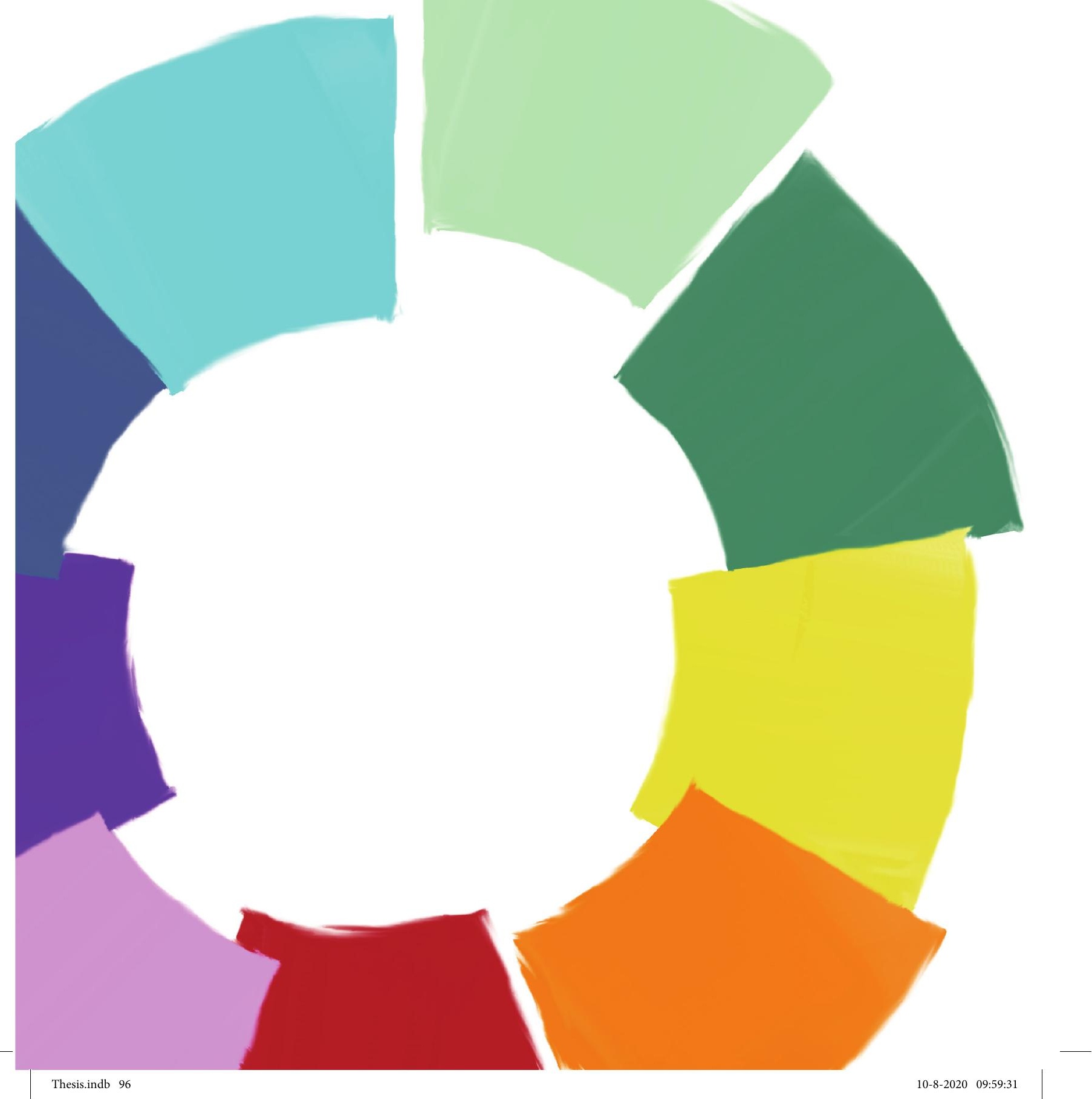




\section{Chapter 6 Development of a Polydimethylsiloxane Microreactor for Performing Multiphase Hydrogenation Reactions at the Single Catalyst Particle Level}

In this Chapter we combine a multiphase system (gas/liquid/solid) and droplet-based microfluidics with optical detection for the analysis of single catalyst particles by safely performing a hydrogenation reaction in a polydimethylsiloxane (PDMS) microreactor. A two-phase segmented flow system of particle-containing droplets is combined with a parallel gas-reactant channel separated from the flow channel by a $50 \mu \mathrm{m}$ thick gas permeable PDMS wall. The developed microreactor system is showcased by monitoring the Pd-catalyzed hydrogenation of methylene blue (MB). A discoloration of blue to brown visualizes the hydrogenation activity happening in a highthroughput fashion on the single $\mathrm{Pd} / \mathrm{SiO}_{2}$ spherical catalyst microparticles, which are encapsulated in $50 \mathrm{~nL}$ sized droplets. By measuring the reagent concentration at various spots along the length of the channel the reaction time can be determined, which is proportional to the residence time in the channel. The developed experimental platform opens new possibilities for single catalyst particle diagnostics.

This work is based on the following manuscript: A.-E. Nieuwelink*, J.C. Vollenbroek*, A.C. Ferreira de Abreu, R.M. Tiggelaar, A. van den Berg, M. Odijk, B.M. Weckhuysen, Single Catalyst Particle Diagnostics in a Microreactor for Performing Multiphase Hydrogenation Reactions, Faraday Discussions, accepted for publication.

* These authors contributed equally. 


\subsection{Introduction}

In the search for more effective catalysts and reaction conditions, the use of highthroughput experimentation to monitor catalyst performance on a single particle level has gained increasing interest over the last decades. ${ }^{[1-4]}$ A growing field of research that has shown great potential for these type of experiments is Lab-on-a-Chip technology, and in particular droplet-based microfluidics. ${ }^{[5-7]}$ When using two immiscible fluids and specific geometries such as a T-junction or flow focusing geometry, droplets can be created by a combination of surface tension and shear forces. ${ }^{[8]}$ Due to the low Reynolds numbers in microfluidic channels, the droplets are typically monodisperse and equally spaced, and do not merge when traveling through the microreactor. With all kinds of external forces (e.g., acoustic, electric, magnetic) the droplets can be manipulated, offering possibilities for more complex chemistry or droplet sorting. ${ }^{[9]}$ Furthermore, the use of droplets compartmentalizes reagents and products, and thus prevents Taylor dispersion. The compartments prevent dispersion along the length of the channel due to the parabolic flow profile, as observed in single phase flow. ${ }^{[8]}$ In these droplets, single catalyst particles can be encapsulated to perform high-throughput screening of their catalytic activity.

Droplet microfluidics aimed for single particle or single cell diagnostics has mainly been used for biochemical essays, such as the study of single cells or rapid screening for the discovery of new enzymes. ${ }^{[10-12]}$ One of the main advantages of droplet microfluidics is that the before-mentioned droplets with a single cell or particle, act as pico-/nanoliter sized reaction vessels i.e. "nanoreactors". The use of optically-transparent reactor materials, such as polydimethylsiloxane (PDMS) or glass, ${ }^{[13]}$ allows for in-situ microand/or spectroscopic measurements.

When we perform a catalytic reaction inside droplets we can detect the activity of each catalyst particle inside a droplet, by measuring for example induced fluorescence, a specific color change or distinctive Raman or IR bands. With an optical or spectroscopic detection method along the channel, the products formed per single particle can be analyzed by measuring all droplets passing the detector. ${ }^{[14]}$ This opens possibilities for the characterization of catalyst particles in liquid phase reactions. However, a large class of heterogeneous catalytic reactions involve not only a liquid phase and a solid phase, but also a gas phase. This class of reactions brings an extra challenge to the field of microfluidics: not much work has been done on the combined control of both liquids, solids and gases. Recent work on the control of multiphase reactions in microfluidic devices has focused on the immobilization of a heterogeneous catalyst either as a packedbed or as a coating on the channel interior. ${ }^{[15-18]}$ Mixed feeds with gas slugs in a liquid flow were used to create a multiphase system. However, the precise control of gas slugs in a liquid flow has proven to be difficult, due to different forces that contribute to the formation and stabilization of liquid droplets. Droplet formation in gas-liquid flow is dependent on more forces than solely the shear force and interfacial tension. ${ }^{[19]}$ Although, adding a surfactant to the liquid phase helped increase flow stability, ${ }^{[20]}$ it is still difficult 
to get monodisperse gas slugs or droplets at fixed flow rates for both phases. ${ }^{[21]}$ This motivates the use of a microreactor with gas-permeable channel walls. In this way, we can form stable liquid "nanoreactor" droplets with encapsulated catalyst particles in an immiscible and non-reactive oil phase. Gases can diffuse through the permeable reactor into the droplets, thereby creating a multiphase (gas/liquid/solid) environment. Gas permeable liquids with low solubility for gases, such as fluorinated oil, can be used as the continuous phase to increase the gas flux towards the droplet.

In this Chapter, a polydimethylsiloxane (PDMS) microreactor has been developed with three parallel channels, as schematically shown in Figure 6.1, to perform gas/ liquid/solid catalytic reactions for the diagnostics of single catalyst particles. PDMS is a flexible polymer that is often used for the fabrication of microfluidic systems, since the fabrication of PDMS microreactors utilizes soft lithography which is relatively cheap and easy. ${ }^{[22]}$ Furthermore, it is known that PDMS has a high permeability to gases such as $\mathrm{H}_{2}$ and $\mathrm{O}_{2}$, which is used to our advantage. The outer channels, as shown in Figure 6.1, are used to flow $\mathrm{H}_{2}$ that permeates through the PDMS walls and reaches the centered liquid channel. In this liquid channel droplets of methylene blue (MB) dissolved in ethanol, containing single particles of a Pd-catalyst, flow in a continuous fluorinated oil phase. As a proof of principle reaction, the hydrogenation of $\mathrm{MB}$ to leuco-MB that discolors from blue to colorless is performed. ${ }^{[23]}$ This reaction is catalyzed by $40 \mu \mathrm{m}$ sized hollow spherical $\mathrm{Pd} / \mathrm{SiO}_{2}$ particles encapsulated in the $\mathrm{MB} /$ ethanol droplets. The droplets that flow through the reactor channel, have identical residence times at any chosen position along the length of the channel. This allows an in-situ analysis of the ongoing reaction, by measuring the color of the droplet at various spots along the length of the channel.

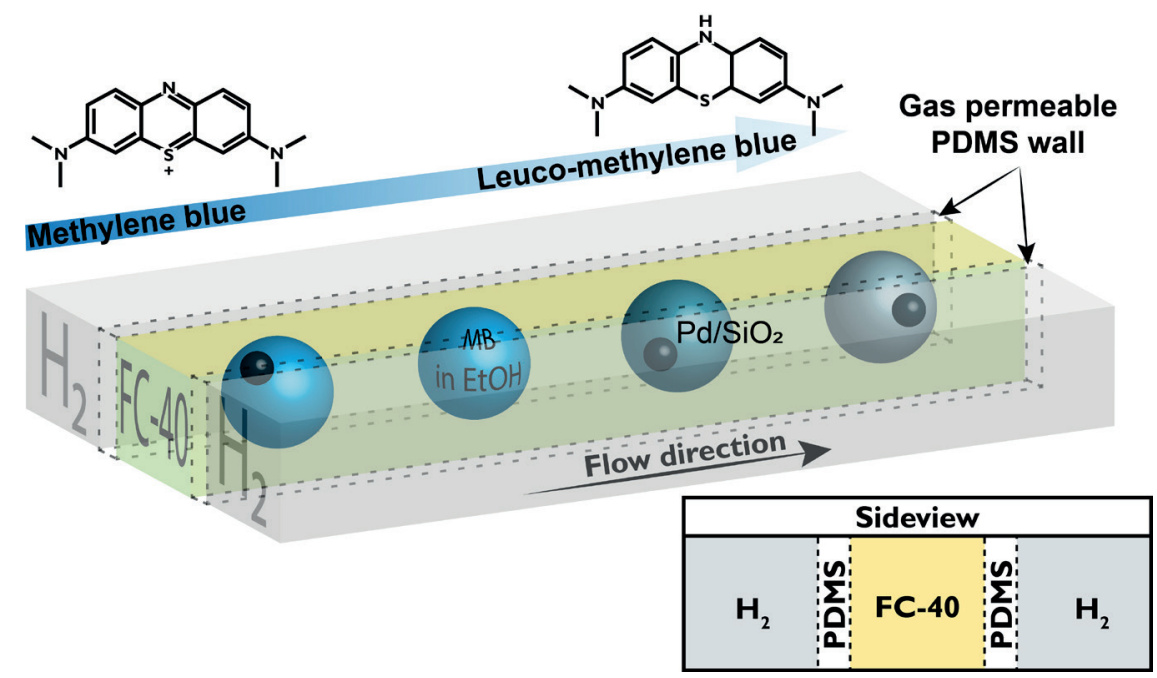

Figure 6.1: Schematic representation of a polydimethylsiloxane (PDMS) microreactor with three parallel channels separated by $50 \mu \mathrm{m}$ thick walls. Hydrogen can diffuse through the walls and hydrogenate methylene blue (MB) to leuco-MB in the presence of $\mathrm{Pd} / \mathrm{SiO}_{2}$ particles in ethanol droplets. 


\subsection{Experimental Section}

\subsubsection{Microreactor Fabrication}

Microreactors were fabricated in PDMS with standard soft- and photolithography methods as described elsewhere. ${ }^{[24]}$ The SU-8 mold was made in the cleanroom of the MESA+ Nanolab at the University of Twente. SU-8 photoresist was spin-coated on a silicon substrate (P-type $\langle 100\rangle$, single side polished, $500 \mu \mathrm{m}$ ) and patterned with a photomask by exposure to UV-light. Structures of $250 \mu \mathrm{m}$ height and width for both the gas channels and the liquid channel were created. The spacing between the gas and liquid channel is $50 \mu \mathrm{m}$. This mold was used to fabricate the inverse replica in PDMS. A liquid mixture of the base- and curing agent (10:1, Sylgard 184, Dow Corning) was degassed and poured over the molds. After thermally curing the PDMS at $60{ }^{\circ} \mathrm{C}$ for $3 \mathrm{~h}$, the layer was peeled from the master. Inlet and outlet holes of $1 \mathrm{~mm}$ diameter are punched in the PDMS after which it was treated in an $\mathrm{O}_{2}$ plasma oven for 2 min and subsequently bonded to another flat layer of PDMS acting as bottom substrate, followed by heating the assembled microreactor to $60{ }^{\circ} \mathrm{C}$ for $30 \mathrm{~min}$. Bonding the structured PDMS slab to the flat bottom slab had to be carried out carefully, to prevent distortion and collapse of the relatively flexible thin PDMS walls. A chip yield of approximately $75 \%$ has been achieved. In total 20 chips have been fabricated and tested.

\subsubsection{Catalyst Preparation and Characterization}

$\mathrm{Pd}$ was deposited on hollow spherical $\mathrm{SiO}_{2}$ particles of $40 \mu \mathrm{m}$ (Materium450) via incipient wetness impregnation of a $6 \mathrm{mM}$ solution of $\mathrm{PdCl}_{2}$ in ethanol. After an equilibrium period of $8 \mathrm{~h}$, the ethanol was slowly evaporated by heating the mixture at $60^{\circ} \mathrm{C}$. After carefully drying the particles, the sample was calcined and reduced in a flow of air and $\mathrm{H}_{2}$ consecutively. The prepared catalyst was characterized with powder X-Ray Diffraction (XRD), Scanning Electron Microscopy (SEM) and $\mathrm{N}_{2}$-physisorption. The crystalline phase of the $\mathrm{Pd} / \mathrm{SiO}_{2}$ catalyst was measured using a Bruker-AXS D2 Phaser equipped with a Co K $\alpha$ radiation source $\left(\lambda=1.78897 \AA\right.$ ) with $2 \theta$ from $10-90^{\circ}$ (increment of 0.1 and scan steps of $1 \mathrm{~s}$ ). SEM images were collected in back-scattered electron mode using a Phenom table top machine. For TEM, a diluted sample of the crushed $\mathrm{Pd} / \mathrm{SiO}_{2}$ in ethanol was drop-casted on lacey copper carbon grids with 300 mesh. The samples were analyzed with a ThermoFisher Scientific Talos F200X equipped with an X-FEG electron source operated at $200 \mathrm{kV}$. EDX images were made using the same apparatus with a Super-XTM EDX detector in STEM mode. A Micromeritics TriStar 300 V6.08 A was used for $\mathrm{N}_{2}$-physisorption measurements at $77 \mathrm{~K}$. The BET equation was used to obtain the specific surface areas. The catalyst particles were added to a syringe with 20 ppm MB in ethanol in a low concentration: $1 \mathrm{mg}$ in $1 \mathrm{~mL}$. This low concentration was needed to increase the possibility of having one particle per droplet, as the amount of particles per droplet follows the Poisson distribution. ${ }^{[25]}$ The blue MB quickly adsorbs on the surface of the silica spheres, leaving blue particles in a pale blue liquid. After entering the microchannel, the droplets with blue $\mathrm{Pd} / \mathrm{SiO}_{2}$ particles flow in between two 
gas channels for $\mathrm{H}_{2}$ supply.

\subsubsection{Microfluidic Reaction: Chemicals and Materials}

The microreactor was designed with three parallel channels, a center-channel for the liquids and two surrounding channels for the gas (all channels have a depth and width of $250 \mu \mathrm{m}$ ). $\mathrm{H}_{2}$ was flushed through the gas channels at $0.75 \mathrm{~mL} / \mathrm{min}$ total flow (using a Bronkhorst mass flow controller of $0-1 \mathrm{~mL} / \mathrm{min}$ ). In the liquid channel $20 \mathrm{ppm} \mathrm{MB}$ in ethanol droplets (with the added catalyst) in a continuous phase of the fluorinated FC40 oil were created. FEP polymer tubing (ID = $500 \mu \mathrm{m}, \mathrm{OD}=1 / 16 ”$ ) from INACOM in combination with Hamilton syringes $(1000 \mu \mathrm{L})$ and a Nemesys (Cetoni) syringe pump were used to create droplets. Flow rates were varied between 2.5 to $20 \mu \mathrm{L} / \mathrm{min}$ and 5 to $20 \mu \mathrm{L} / \mathrm{min}$ for the ethanol (dispersed phase) and fluorinated oil (continuous phase), respectively. Microscopy images were made and data acquisition was done with a Zeiss Axio Zoom.V16 microscope using bottom illumination, equipped with a PlanNeoFluar Z 1x zoom objective and an Axiocam 105 color camera, as shown in Figure 6.2.

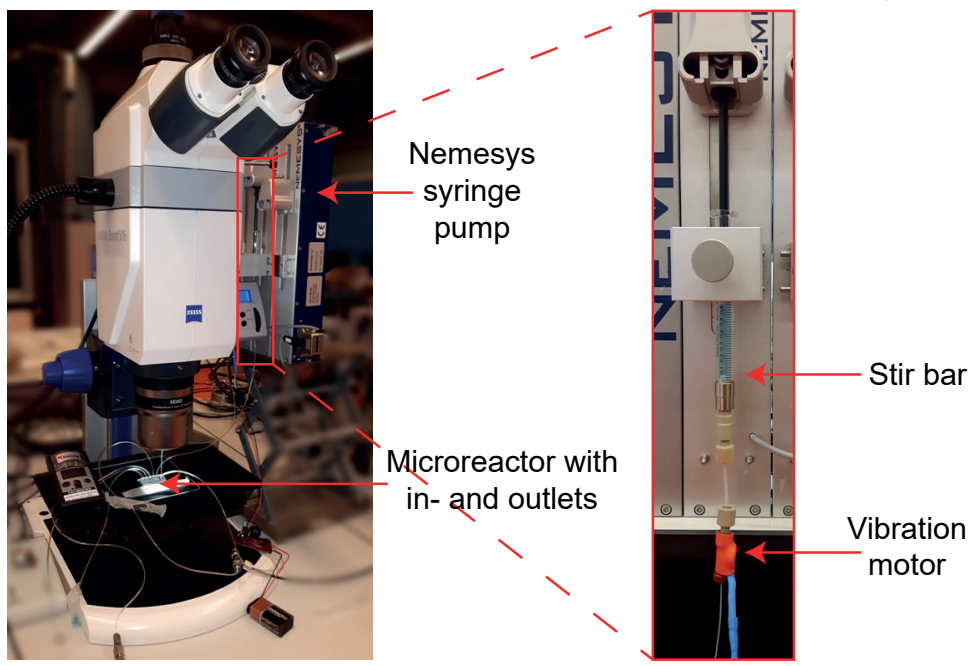

Figure 6.2: Images of the microscope setup with vertical syringe pump, syringe with stir bar and vibration motor for optimal conditions to encapsulate solid particles in droplets.

\subsubsection{Matlab Data Analysis}

All movies acquired were screened for the presence of catalyst particles in a droplet, using a small window at three positions of the microreactor (start, middle, and end). Figure 6.3 shows three examples of particles found at these locations. To find the center of the particles, all red values in the window are added column wise and then multiplied by -1 . The result is the bottom row of images in Figure 6.3. Here it can be seen that the position of the particle results in a peak, because the red values are significantly lower for the particle then for the background. The presence of a droplet interface also results in a peak, but by looking at the width of the peak as well, they can be filtered from actual particles. The findpeaks function in Matlab is used to find the position of the particle peak. With this position a line (yellow line in Figure 6.3) can be drawn over the middle 
Start
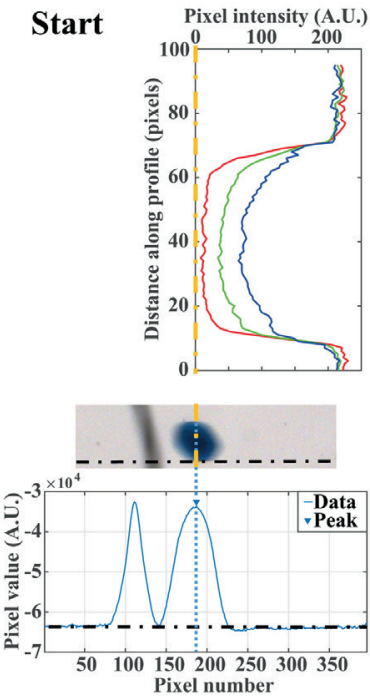

Mid
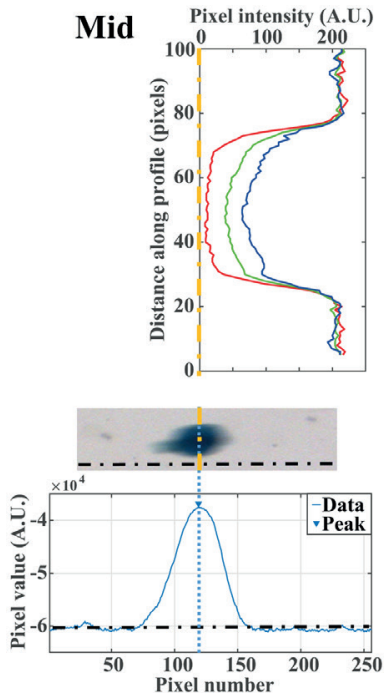

End

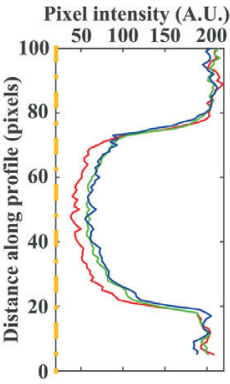

Figure 6.3: Visual representation of the data analysis. The found particles in the detection window are shown in the middle row. The bottom row shows the Red values of the window added column wise and then multiplied by -1. This results in the peaks as shown in the bottom row. The droplet interface also results in a peak but by filtering on the width of the peak the particle can still be detected with the findpeaks function in Matlab. With the position of the peak known, a line can be drawn across the particle (orange line) and along this line the Red/Green/Blue values of the particle are plotted. To obtain a single datapoint for the R, G, and $B$ value of a particle, the lowest 10 values of each color are added and averaged.

of the particle, plotting the Red/Green/Blue values along this line, as seen in the top row of images in Figure 6.3.

\subsection{Results and Discussion}

\subsubsection{Microreactor Design and Fabrication}

The PDMS microreactor, as shown in Figure 6.4, was designed to benefit from the gas-permeability of PDMS: a thin wall of $50 \mu \mathrm{m}$ between the gas and the droplet channel allows for fast diffusion of $\mathrm{H}_{2}$ to the liquid droplets with reactant and catalyst. The hydrogenation reaction can only take place when the three phases meet (the solid catalyst particle, the liquid $\mathrm{MB}$ in ethanol and the gaseous $\mathrm{H}_{2}$ ) and therefore starts when the catalyst particle-containing droplets enter the liquid microchannel (250x250 $\mu \mathrm{m}$ width and depth) surrounded by two $\mathrm{H}_{2}$ gas channels with similar cross-sectional dimensions. Ethanol droplets (containing $\mathrm{MB}$ ) are created via a flow focusing geometry using a fluorinated hydrocarbon oil FC-40 as continuous phase. The droplets of $\sim 50 \mathrm{~nL}$ travel through the microreactor in $45 \mathrm{~s}$ with a total flow rate of $7.5 \mu \mathrm{L} / \mathrm{min}$.

For the hydrogenation to take place, the gaseous $\mathrm{H}_{2}$ has to travel from the gas channels through the PDMS wall and the fluorinated oil (FC-40) continuous phase before it reaches the ethanol droplet. It is important that the $50 \mu \mathrm{m}$ thick PDMS wall does not interfere too much with the $\mathrm{H}_{2}$ diffusion in ethanol, to prevent a change in reaction kinetics due to mass transport limitations. Figure 6.5a shows which factors influence the $\mathrm{H}_{2}$ concentration in the ethanol droplet. For a flow with spherical droplets, the continuous 
a)

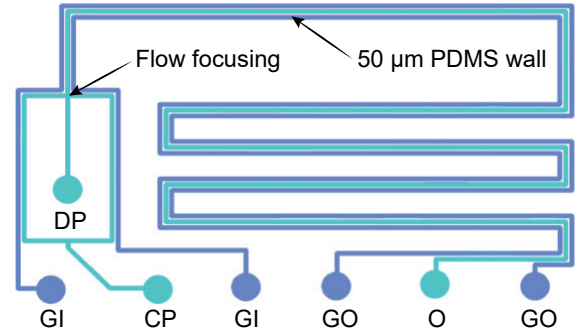

b)

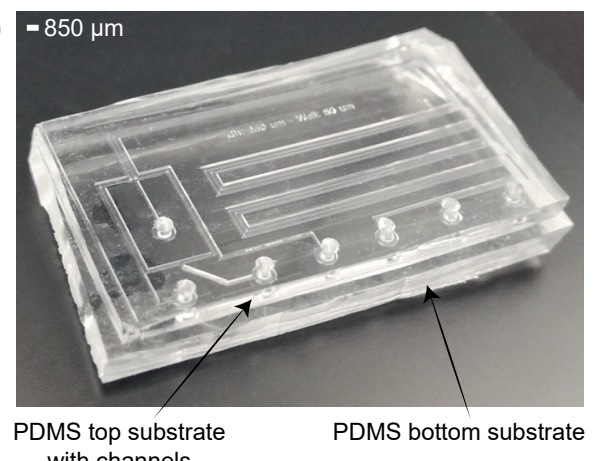

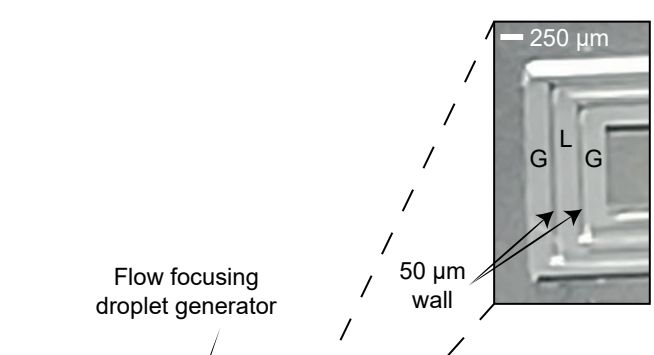

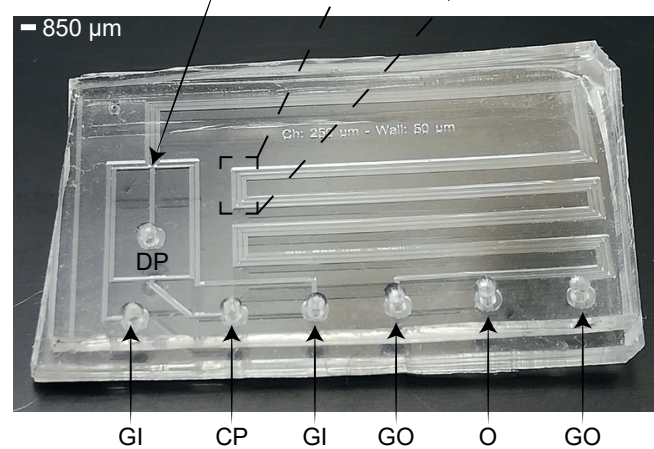

Figure 6.4: A schematic representation (a) and images (b) of the polydimethylsiloxane (PDMS) microreactor (2xl cm length and width) and of the channel system showing the Gas Inlet (GI), Continuous Phase inlet (CP), Droplet Phase inlet (DP), Gas Outlet (GO) and fluidic Outlet (O). In a zoom-in of the Gas (G) and Liquid (L) channels, the $50 \mu \mathrm{m}$ thin PDMS wall that separates the gas and liquid channels is shown. All channels are $250 \mu \mathrm{m}$ wide and deep.

phase is wetting the channel walls, whereas the droplet phase is non-wetting. This leads to the formation of a thin stagnant film of oil between the ethanol droplet and the PDMS wall. Figure $6.5 \mathrm{~b}$ shows that the droplets in our microreactor system are slugs rather than spherical droplets. ${ }^{[26]}$ Based on the small contact angle between the non-wetting slug and the wall, we assume that there is a nm thick (but invisible) layer of FC-40 in between the droplet and the PDMS. Since, FC-40 has a high $\mathrm{H}_{2}$ gas permeability (similar to PDMS) ${ }^{[27]}$, consequently there is no diffusion limitation caused by the FC-40 film. From this, we concluded the following: $\mathrm{H}_{2}$ must first diffuse into and through the PDMS wall before it can enter the ethanol droplet containing the catalyst. From Table 6.1, it can be observed that the diffusivity (D) of $\mathrm{H}_{2}$ through PDMS

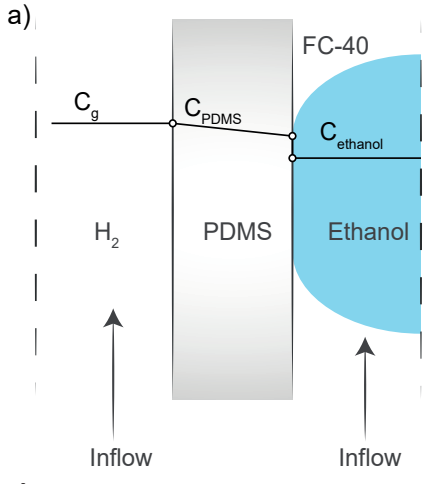

b)

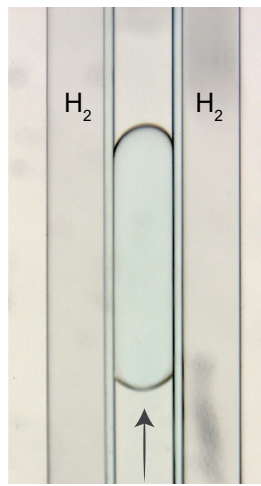

Figure 6.5: Simplified/schematic representation of the $\mathrm{H} 2$ concentrations in the different compartments of the microreactor (a) and a microscope image of one droplet (b). The ethanol droplets partially wet the polydimethylsiloxane (PDMS) wall and are therefore considered as 'slugs'. Due to the high diffusivity of hydrogen through PDMS $\left(1.4^{*} 10^{4}\right.$ $\left.\mathrm{m}^{2} / \mathrm{s}\right)$, with respect to ethanol $\left(1.49^{*} 10^{2} \mathrm{~m}^{2} / \mathrm{s}\right)$, the limiting diffusion step is for hydrogen to enter the ethanol droplet. 
Table 6.1: Diffusion (D) and solubility (S) parameters of $\mathrm{H}_{2}$ in polydimethylsiloxane (PDMS) and ethanol.

\begin{tabular}{c||c|c} 
& $\mathrm{D}\left(\mathrm{m}^{2} / \mathrm{s}\right)$ & $\mathrm{S}\left(\times 10^{5} \mathrm{~Pa}\right)$ \\
\hline \hline PDMS & $1.40 \times 10^{4[29]}$ & $20.3^{[29]}$ \\
\hline Ethanol & $1.49 \times 10^{2[30]}$ & $452^{[31]}$
\end{tabular}

is a factor 100 higher than of $\mathrm{H}_{2}$ through ethanol. Inside the PDMS wall and the ethanol droplet, the solubility follows Henry's law. The solubility (S) parameter of $\mathrm{H}_{2}$ is 200 times higher in ethanol compared to PDMS. A high diffusivity but low solubility of $\mathrm{H}_{2}$ in PDMS results in fast transport of the hydrogen through the PDMS membrane. In ethanol, the $\mathrm{H}_{2}$ diffusivity is lower, but its solubility is higher compared to PDMS, leading to $\mathrm{H}_{2}$-saturated ethanol droplets. Therefore, it is assumed that the PDMS is not introducing significant diffusion limitations. Within the droplets, chaotic advection causes fast mixing ${ }^{[28]}$, leading to a homogeneous and constant $\mathrm{H}_{2}$ concentration. Figure 6.5a sketches the situation where the ethanol droplet is completely saturated with $\mathrm{H}_{2}$ and homogeneously mixed, therefore there is a discontinuity in the simplified representation at the PDMS-droplet interface. When the ethanol concentration in the droplet becomes lower because the $\mathrm{H}_{2}$ reacts with $\mathrm{MB}$ on the Pd catalyst, $\mathrm{H}_{2}$ can diffuse from the PDMS and dissolve into the ethanol droplet again.

\subsubsection{Catalytic Hydrogenation of Methylene Blue}

The developed PDMS microreactor was tested for the hydrogenation of MB (blue) into leuco-MB (colorless). As catalyst, we have opted for $\mathrm{Pd}$ supported on hollow $\mathrm{SiO}_{2}$ spheres of $40 \mu \mathrm{m}$ in diameter. We have selected Pd as active metal because of its known activity towards hydrogenation reactions. An added benefit is its stability in air. After a reduction step, the formed Pd particles are not prone to oxidation and can, therefore, easily be transferred through or stored in air. Our microreactor setup lacks options for in-situ catalyst reduction steps, thus a stable and robust catalyst system is vital. The hollow core of the silica particles lowers their weight by a factor 10 approximately (bulk density of 0.2 $\mathrm{mg} / \mathrm{L}$ vs. skeletal density $2.5 \mathrm{mg} / \mathrm{L}$ ), to improve their flow through the microchannel. In general, catalyst particles with much higher densities than the surrounding liquid have difficulty moving inside microfluidic channels, as they tend to sink to the bottom due to gravity. ${ }^{[32]}$ Once stuck at the channel bottom, heavy catalyst particles cannot easily be removed due to the absence of flow at the wall. Since catalyst supports often have a higher density compared to the liquid reactants, this sinking is an important factor in microfluidic single particle diagnostics. The prepared catalyst was characterized with SEM, XRD, TEM and $\mathrm{N}_{2}$-physisorption. SEM and TEM images can be found in Figures 6.6a and b. With SEM, the external surface and morphology was investigated. It was found that the structure and shape of the spherical $\mathrm{SiO}_{2}$ particles was intact after the preparation of the catalyst. The $40 \mu \mathrm{m}$ porous $\mathrm{SiO}_{2}$ microparticles were too big to analyze with TEM, but after crushing them, the remaining fragments could be analyzed to determine the Pd dispersion and nanoparticle size. On average, the Pd nanoparticles 
a)

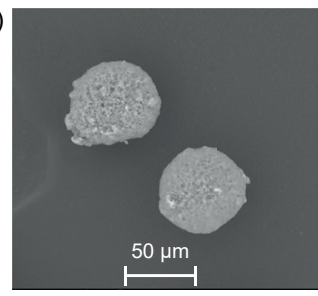

c)

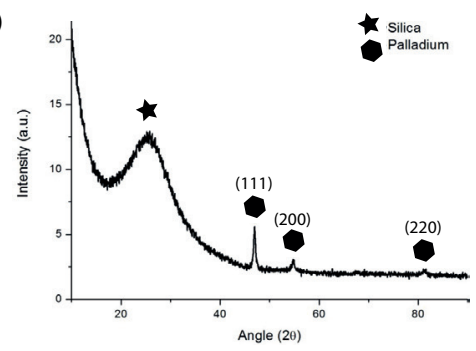

b)
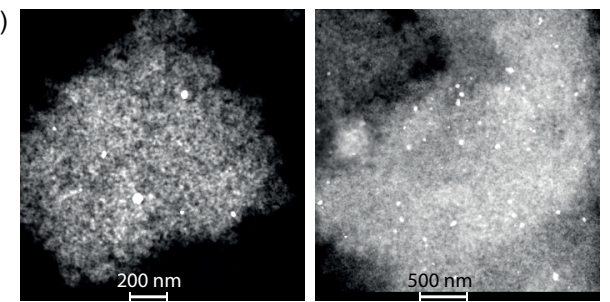

d)

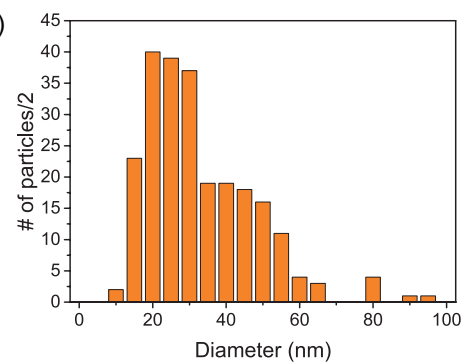

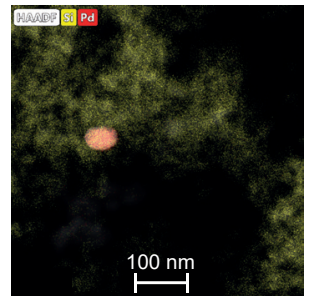

1

Figure 6.6: Scanning electron microscopy (SEM) images in backscatter mode of the catalyst prepared (a) show an intact spherical shape and large Pd clusters. TEM images in STEM mode (b), of the crushed spheres show homogeneously distributed Pd NP. In the X-ray diffraction (XRD) pattern (c) peaks corresponding to silica and Pd are assigned. The particle size distribution (d) as determined from multiple TEM images is large. Two sides were measured of each of the particles: the values on the Y-axis should be divided by two for the actual number of particles measured.

are $-25-30 \mathrm{~nm}$. The particle size distribution is large, but their dispersion over the $\mathrm{SiO}_{2}$ support is homogeneous, as can be seen in Figure 6.6d. In the XRD (Figure 6.6c), diffraction peaks allocated to crystalline Pd were found, and with the Scherrer equation using the Full Width Half Maximum (FWHM) of the XRD peaks, an average crystallite size of $\sim 25 \mathrm{~nm}$ could be determined. The BET surface area of the particles was calculated to be $\sim 180 \mathrm{~m}^{2} / \mathrm{g}$.

For performing the hydrogenation reaction inside the droplet microreactor, a setup was built with a vertically mounted syringe pump to benefit from gravitational forces acting on the catalyst particles. A shaker was connected to the tip of the syringe, inducing vibrations in the tubing and a stir bar was added to the syringe to disperse the particles in the ethanol solution. Both measures are a precaution against particle sedimentation in the syringe or tubing. A detailed image of the setup is given in Figure 6.2. The droplets were followed with optical microscopy to monitor a color change of all droplets that contained catalyst particles.

As part of the experimental setup, the catalyst particles were added to the MB-ethanol syringe prior to starting the flows. Since no reaction can happen before the particles have entered the microreactor, due to the absence of $\mathrm{H}_{2}$ in the glass syringe, we have a welldefined starting time for the reaction $\left(\mathrm{t}_{0}\right)$. Interestingly, after adding the $\mathrm{Pd} / \mathrm{SiO}_{2}$ to a 20 ppm solution of MB in ethanol, a rapid color change of the fresh catalyst was observed from brown to blue, due to the adsorption of MB. Figure 6.7 shows two microscopy images of the catalyst before and after adsorption of MB. This color change is advantageous for the catalyst characterization: the blue particles should return to their original brown 
a)

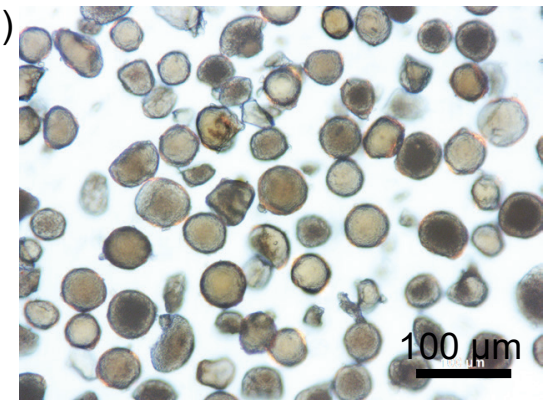

b)

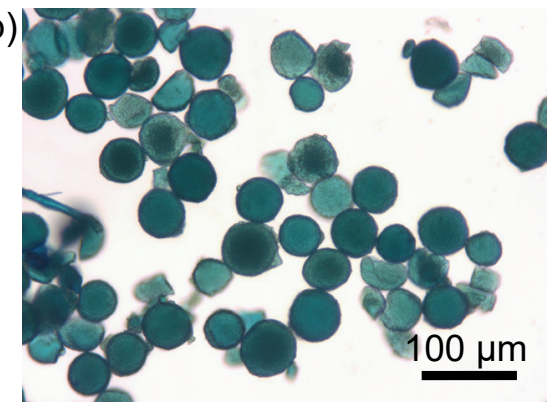

Figure 6.7: Two optical images of the $\mathrm{Pd} / \mathrm{SiO}$ catalyst before (a) and after (a) adding them to 20 ppm methylene blue (MB) in ethanol. The blue color is caused by adsorption of $\mathrm{MB}$ on the surface of the solid particles.

color upon hydrogenation. This color change effect is expected to be more pronounced compared to a discoloration of the light blue color of the MB droplet itself and therefore easier to detect.

As a proof of concept experiment, droplets containing blue $\mathrm{Pd} / \mathrm{SiO}_{2}$ particles were followed over time, without any liquid flow, leading to stagnant droplets. It can be seen in Figure 6.8 that after starting the $\mathrm{H}_{2}$ flow, the particles rapidly discolor from blue to brown and the Red/Green/Blue (RGB) values reach a plateau over time. During the experiments $\mathrm{a}_{2}$ sensor (Industrial Scientific Pro Gas Badge) is put next to the microreactor for safety reasons. The minimum amount it can detect is $1 \mathrm{ppm}$ and during all experiments, this level was never reached.

These results show the working principle of the PDMS hydrogenation microreactor: $\mathrm{H}_{2}$ can diffuse through the PDMS wall and dissolves in the reactant droplet. However, for single particle diagnostics, these experiments should be performed in flow. Therefore, the same hydrogenation of $\mathrm{MB}$ was performed at a total flow of $7.5 \mu \mathrm{L} / \mathrm{min}$, leading to a droplet formation rate of 1 droplet/s and a residence time of $45 \mathrm{~s}$ in the microchannel. The analysis of particles in flow is more difficult compared to the static experiment. Therefore, three monitoring regions for characterization were defined at the start, middle and the end of the microchannel (Figure 6.9a). In this way, all particles can be analyzed for three different reaction times. Figure 6.9c shows two sets of images of $\mathrm{Pd} / \mathrm{SiO}_{2}$ in a MB-ethanol droplet recorded at the start and end regions.

Indeed, the blue color of the particles at the start of the channel has disappeared towards the end of the channel. From these results, we conclude that $\mathrm{H}_{2}$ also permeates the PDMS wall, dissolves in the ethanol droplet and participates in the reaction when the droplets experience a flow. Thus, our microreactor design is working: within the 45 $\mathrm{s}$ residence time $\left(\mathrm{t}_{\mathrm{r}}\right)$ in the microchannel, the MB hydrogenates to the colorless leucoMB. To support these findings, control experiments without $\mathrm{H}_{2}$ or active catalyst are performed: the particles stay blue during their residence in the microchannel. The color change is most visible for the more concentrated $\mathrm{MB}$, adsorbed on the particles. Empty droplets contain the same initial concentration of $\mathrm{MB}$ as droplets containing a particle. 
a)

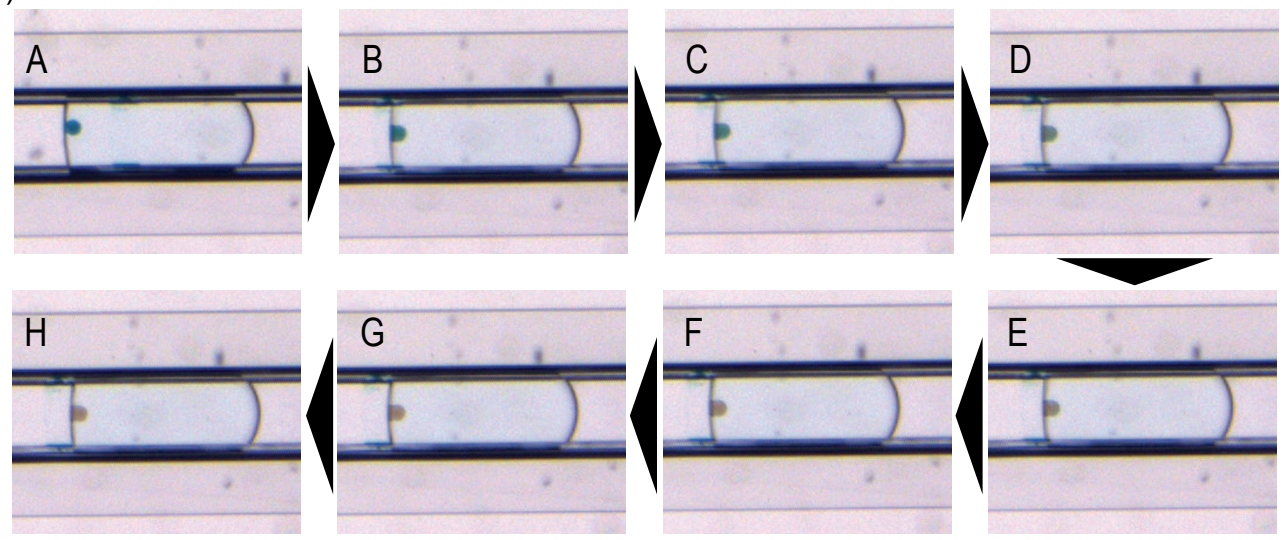

b)

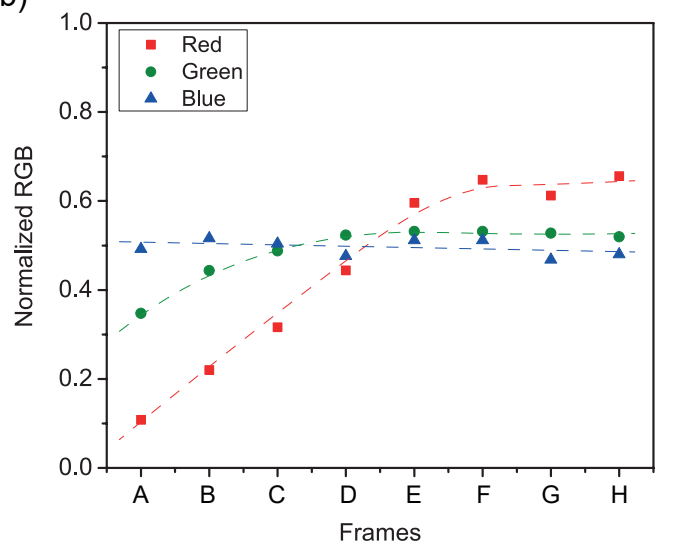

Figure 6.8: Separate frames (a; with an interval of $1.3 \mathrm{~s}$ each, going from A to $\mathrm{H}$ ) show a rapid particle discoloration from blue to brown when the droplets are exposed to a $\mathrm{H}_{2}$ flow via the adjacent gas channels. The plot (b) shows the changes in the RGB channels per frame.

However, the concentration in the droplets is too low to be visible in the images and both empty droplets and particle containing droplets appear 'white' and have no discernable color change in the RGB level. With the presented setup, the encapsulation of particles in droplets was relatively straightforward. However, fluctuating concentrations of particles near the inlet of the microreactor often led to multiple particles per droplet.

For the flow experiments, a statistically relevant analysis was performed by monitoring the red-green-blue (RGB) levels of the acquired movies in Matlab. The movies were screened for the presence of catalyst particles in a droplet using a small window at three positions on the microreactor (start, middle, and end) as indicated in Figure 6.9a. Figure 6.3 in the experimental section shows three examples of particles found at these different locations. Matlab processing was used to find the frames containing particles. The RGB values of all particles were collected using a line profile positioned perpendicular to the flow direction. From these line profiles, it can be clearly seen that the particles have lower RGB values than the background. The ten lowest RGB values of each particle 
a)

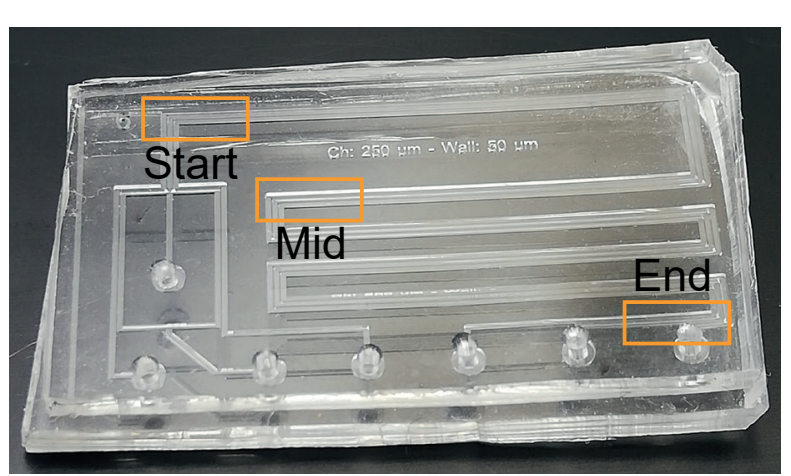

c)

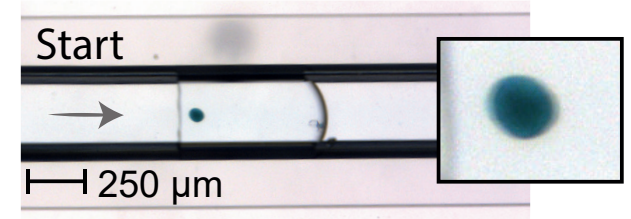

b)

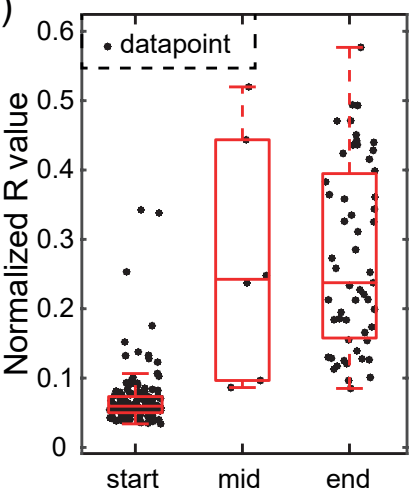

Figure 6.9: The measurement positions on the microreactor are shown in (a). Normalized red-green-blue (RGB) levels with respect to the background polydimethylsiloxane (PDMS) signal of particles in droplets at the start, mid and end of the reaction channel are given (b). Droplets containing particles appear blue at the start of the reaction channel (c, left) and brown near the end of the reaction channel (c, right) due to hydrogenation of methylene blue (MB) to the colorless leuco-MB. The arrow indicates the flow direction. The number of particles measured at the start, mid and end regions are 139, 6 and 57 respectively.

are summed and averaged to obtain the RGB value for a particle at each position. This tracking allowed for an evaluation of the RGB levels for each particle. Figure 6.9b shows a boxplot of red levels for multiple particles at the start, middle and at the end of the flow channel. The datapoints obtained from the Matlab script are plotted on top of the boxplot. Only the red value is shown, as that is the value with the most distinctive change upon discoloration, as shown in Figure 6.8. The values are normalized with respect to a reference position on the PDMS, denoted as background. From this plot, the color change of $\mathrm{Pd} / \mathrm{SiO}_{2}$ catalyst particles flowing through the microchannel is clearly visible. At the start, the blue particles show low red values, whereas the brown particles at the middle and end show high red values. For the middle region, only 6 particles could be measured, leading to less sound statistics. However, due to the similar red color value in the boxplot at the middle compared to the end position, it is concluded that the hydrogenation has already been completed at the middle position $(54 \mathrm{~mm}$ after droplet formation and exposure to $\mathrm{H}_{2}$ ).

Interestingly, this hydrogenation is a fast reaction and does not need the full residence time of $45 \mathrm{~s}$ (when using a total flow of $7.5 \mu \mathrm{L} / \mathrm{min}$ ) in the microreactor to be completed. Due to the gradual color change from blue to brown, it is difficult to pinpoint the exact position in the microreactor where the hydrogenation is completed, but it is approximated that the reaction is completed within $10 \mathrm{~s}$, because the red value of the particle does not change after this point, as shown in Figure 6.8, from frame F onwards. For a total flow 

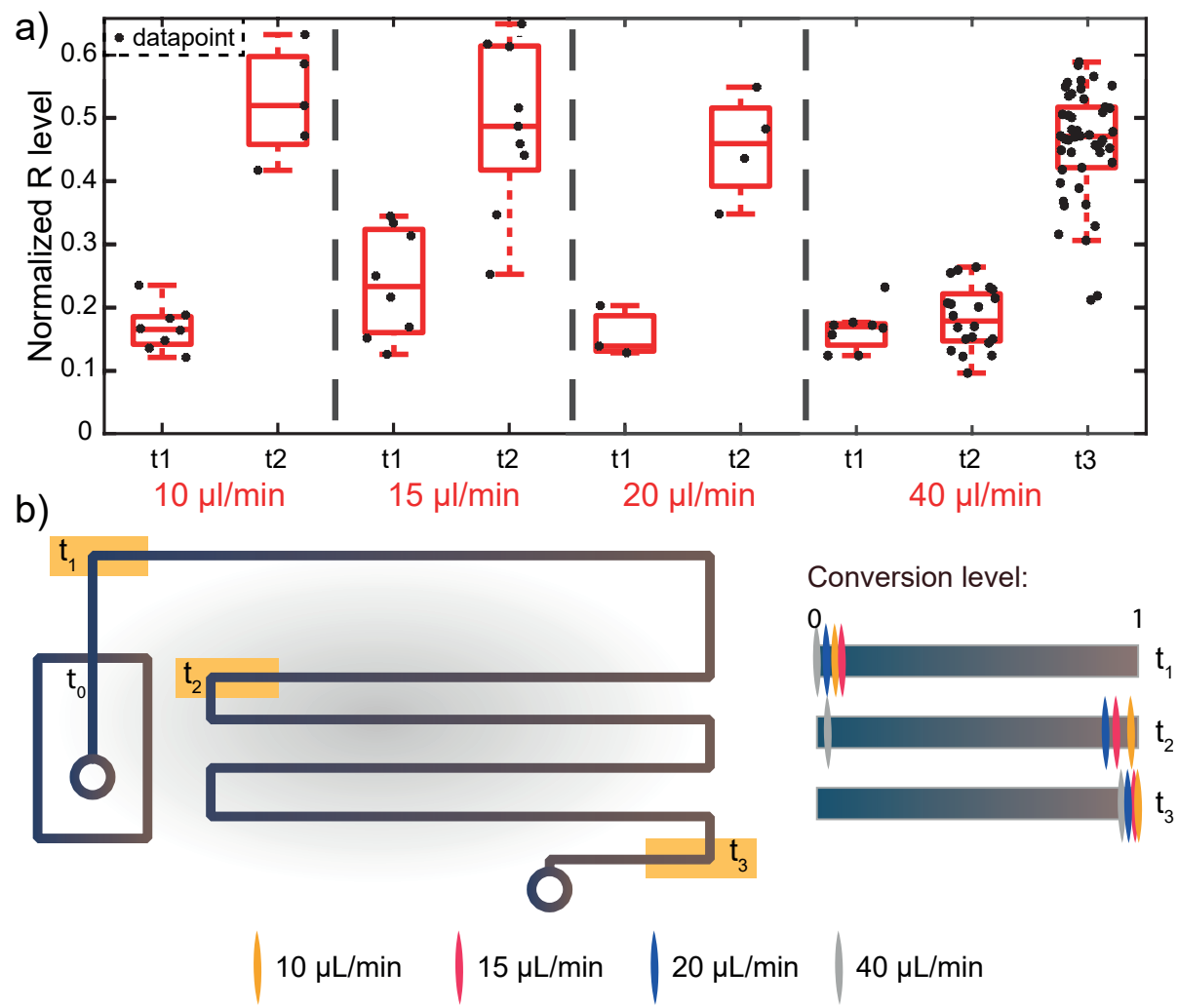

Figure 6.10: The start ( $\mathrm{t}_{1}: 6.8 \mathrm{~mm}$ after droplet formation), middle ( $\mathrm{t}_{2}: 53.8 \mathrm{~mm}$ after droplet formation) and end $(t: 123.4 \mathrm{~mm}$ after droplet formation) regions were analyzed at different flow rates (a). The total flow rates are given, the ratio of the Continuous Phase (CP) vs. Droplet Phase (DP) phase was 1:1. For the fastest rate (total flow of $40 \mu \mathrm{L} / \mathrm{min}$ ) also the end region was analyzed. The graphical representation of the results (b) shows that at higher flow rates, the reaction is completed more down-stream in the microchannel.

of $7.5 \mu \mathrm{L} / \mathrm{min}, \sim 1$ droplet per second passes the analysis region. To have a high particle throughput, it is best to use the highest flowrate possible. Increasing the flowrate to 40 $\mu \mathrm{L} / \mathrm{min}$ leads to larger, but less stable droplets (Movie M4), but the hydrogenation still takes place and the droplet size does not seem to affect the reaction kinetics. Figure 6.10 shows the red color values for the start and middle of the reaction channel, for four different flow rates. It can be seen that up to a total flow of $20 \mu \mathrm{L} / \mathrm{min}(10 / 10$, w/o), the reaction has completed at the mid region of the microreactor. For a total flow of $40 \mu \mathrm{L} /$ min, the residence time of a droplet in the microreactor is decreased to $8.5 \mathrm{~s}$. At this flow rate, the full channel length is needed for completion of the reaction, meaning that the reaction time for the hydrogenation of $\mathrm{MB}$, at these conditions, is $\sim 8 \mathrm{~s}$. Therefore, the end position is also measured at this flow rate.

\subsection{Conclusions}

In this Chapter, we present a proof of concept of a polydimethylsiloxane (PDMS) microreactor for high-throughput, multiphase catalytic reactions, demonstrating the liquid-phase hydrogenation of methylene blue (MB). A liquid channel with $\mathrm{Pd} / \mathrm{SiO}_{2}$ 
particles of $40 \mu \mathrm{m}$ encapsulated in droplets containing $20 \mathrm{ppm} \mathrm{MB}$ in ethanol are flanked by two gas channels with $\mathrm{H}_{2}$, separated with $50 \mu \mathrm{m}$ PDMS walls. Due to the high permeability of PDMS, $\mathrm{H}_{2}$ diffuses into the liquid channels and facilitates the Pdcatalyzed hydrogenation of $\mathrm{MB}$ at room temperature. In $\sim 8 \mathrm{~s}$ the reaction completes, as concluded from the discoloration of the particles in droplets going from blue (due to adsorbed $\mathrm{MB}$ ) to brown (their original color). Droplets containing single particles could be analyzed individually to allow for the diagnostics of multiple heterogeneous catalyst particles, as opposed to the use of packed-bed or wall-coated reactors. This newly developed microreactor has, therefore, proven to be useful for the single particle diagnostics of catalyst particles in complex multiphase reactions. With this we have a new diagnostics tool in hand to improve the statistical relevance of single catalyst particle analysis and bridge the gap towards bulk characterization methods. In addition, a safe and relatively easy method has been demonstrated to work with $\mathrm{H}_{2}$, as no $\mathrm{H}_{2}$ leak was detected by a commercial sensor during all the experiments and only small amounts of gas are involved. Furthermore, by placing the PDMS chip on a heating stage, reactions at elevated temperatures can be performed. The thermal stability of crosslinked PDMS allows for heating up to $400{ }^{\circ} \mathrm{C}$ before it starts to degrade ${ }^{[33]}$, but in this situation more problems rise, such as the boiling of solvents or a non-uniform heating of the microreactor. In addition, the optical properties of PDMS allow for monitoring reactions with visible (laser) light, therefore allowing to perform in-line UV-vis or Raman spectroscopy. These techniques should be optimized in a way that they can work fast and can detect individual droplets at high flow rates. Finally, more sophisticated microreactor designs with microfluidic droplet sorters can be implemented to sort the catalyst particles of interest.

\subsection{Acknowledgements}

We thank Johan Bomer (University of Twente) for his contributions to this research, Nynke Krans (Utrecht University, UU) for TEM measurements and Fang Liu (UU) for the help and related discussions.

\subsection{References}

[1] B. Sun, X. Meng, S. Wang, S. Sun, F. Xiao, Acta Physico-Chimica Sin. 2006, 22, 441-444.

[2] H. Fang, Q. Xiao, F. Wu, P. E. Floreancig, S. G. Weber, J. Org. Chem. 2010, 75, 5619-5626.

[3] T. Zech, G. Bohner, J. Klein, Catal. Today 2005, 110, 58-67.

[4] F. G. Welsch, K. Stöwe, W. F. Maier, ACS Comb. Sci. 2011, 13, 518-529.

[5] G. M. Whitesides, Nature 2006, 442, 368-373.

[6] S. Haeberle, R. Zengerle, Lab Chip 2007, 7, 1081-1220.

[7] E. Y. Basova, F. Foret, Analyst 2015, 140, 22-38.

[8] X. Casadevall, Chem. Commun. 2011, 47, 1936-1942.

[9] H.-D. Xi, H. Zheng, W. Guo, A. M. Ganan-Calvo, Y. Ai, C.-W. Tsao, J. Zhou, W. Li, Y. Huang, N.-T. Nguyen, S. H. Tan, Lab Chip 2017, 17, 751-771.

[10] R. M. Schoeman, E. W. M. Kemma, F. Wolbers, A. van den Berg, Electrophoresis 2014, 35, 385-392.

[11] H. A. Bunzel, X. Garrabou, M. Pott, Curr. Opin. Struct. Biol. 2018, 48, 149-156.

[12] A. Autour, M. Ryckelynck, Micromachines 2017, 8, 128-149.

[13] D. Cai, A. Neyer, R. Kuckuk, H. M. Heise, J. Mol. Struct. 2010, 976, 274-281.

[14] R. Burger, L. Amato, A. Boisen, Biosens. Bioelectron. 2016, 76, 54-67.

[15] H. P. L. Gemoets, Y. Su, M. Shang, V. Hessel, R. Luque, T. Noël, Chem. Soc. Rev. 2016, 45, 83-117. 
[16] G. Takei, T. Kitamori, H. Kim, Catal. Commun. 2005, 6, 357-360.

[17] M. Liu, X. Zhu, R. Chen, Q. Liao, H. Feng, L. Li, Chem. Eng. J. 2016, 301, 35-41.

[18] J. Kobayashi, Y. Mori, K. Okamoto, R. Akiyama, M. Ueno, T. Kitamori, S. Kobayashi, Science 2004, 304, 1305-1309.

[19] P. Garstecki, I. Gitlin, W. Diluzio, G. M. Whitesides, E. Kumacheva, H. A. Stone, Appl. Phys. Lett. 2004, 85, 2649-2651.

[20] J. Tan, S. W. Li, K. Wang, G. S. Luo, Chem. Eng. J. 2009, 146, 428-433.

[21] A. Günther, S. A. Khan, M. Thalmann, F. Trachsel, K. F. Jensen, Lab Chip 2004, 4, 278-286.

[22] J. C. Mcdonald, D. C. Duffy, J. R. Anderson, D. T. Chiu, H. Wu, O. J. A. Schueller, G. M. Whitesides, Electrophoresis 2000, 21, 27-40.

[23] Y.-N. Liu, X. Zhou, X. Wang, K. Liang, Z.-K. Yang, C.-C. Shen, M. Imran, S. Sahar, A.-W. Xu, RSC Adv. 2017, 7, 30080-30085.

[24] D. B. Weibel, W. R. Diluzio, G. M. Whitesides, Nat. Rev. Microbiol. 2007, 5, 209-218.

[25] D. J. Collins, A. Neild, A. DeMello, A. Liu, Y. Ai, Lab Chip 2015, 15, 3439-3459.

[26] H. Song, D. L. Chen, R. F. Ismagilov, Angew. Chem. Int. Ed. 2006, 45, 7336-7356.

[27] S. K. O. Ntwampe, C. C. Williams, M. S. Sheldon, African J. Biotechnol. 2010, 9, 1106-1114.

[28] H. Song, M. R. Bringer, J. D. Tice, C. J. Gerdts, R. F. Ismagilov, Appl. Phys. Lett. 2003, 83, 4664-4666.

[29] T. C. Merkel, V. I. Bondar, K. Nagai, B. D. Freeman, I. Pinnau, J. Polym. Sci. B Polym. Phys. 2000, 38, 415-434.

[30] K. Sporka, J. Hanika, V. Ruzicka, M. Halousek, Collect. Czechoslov. Chem. Commun 1971, 36, $2130-2136$. [31] M. S. Wainwrlght, T. Ahn, D. L. Trimm, N. W. Cant, J. Chem. Eng. Data 1987, 32, 22-24.

[32] D. Huh, J. H. Bahng, Y. Ling, H. H. Wei, O. D. Kripfgans, J. B. Fowlkes, J. B. Grotberg, S. Takayama, Anal. Chem. 2007, 79, 1369-1376.

[33] J. González-Rivera, R. Iglio, G. Barillaro, C. Duce, M. R. Tinè, Polymers 2018, 10, 616. 


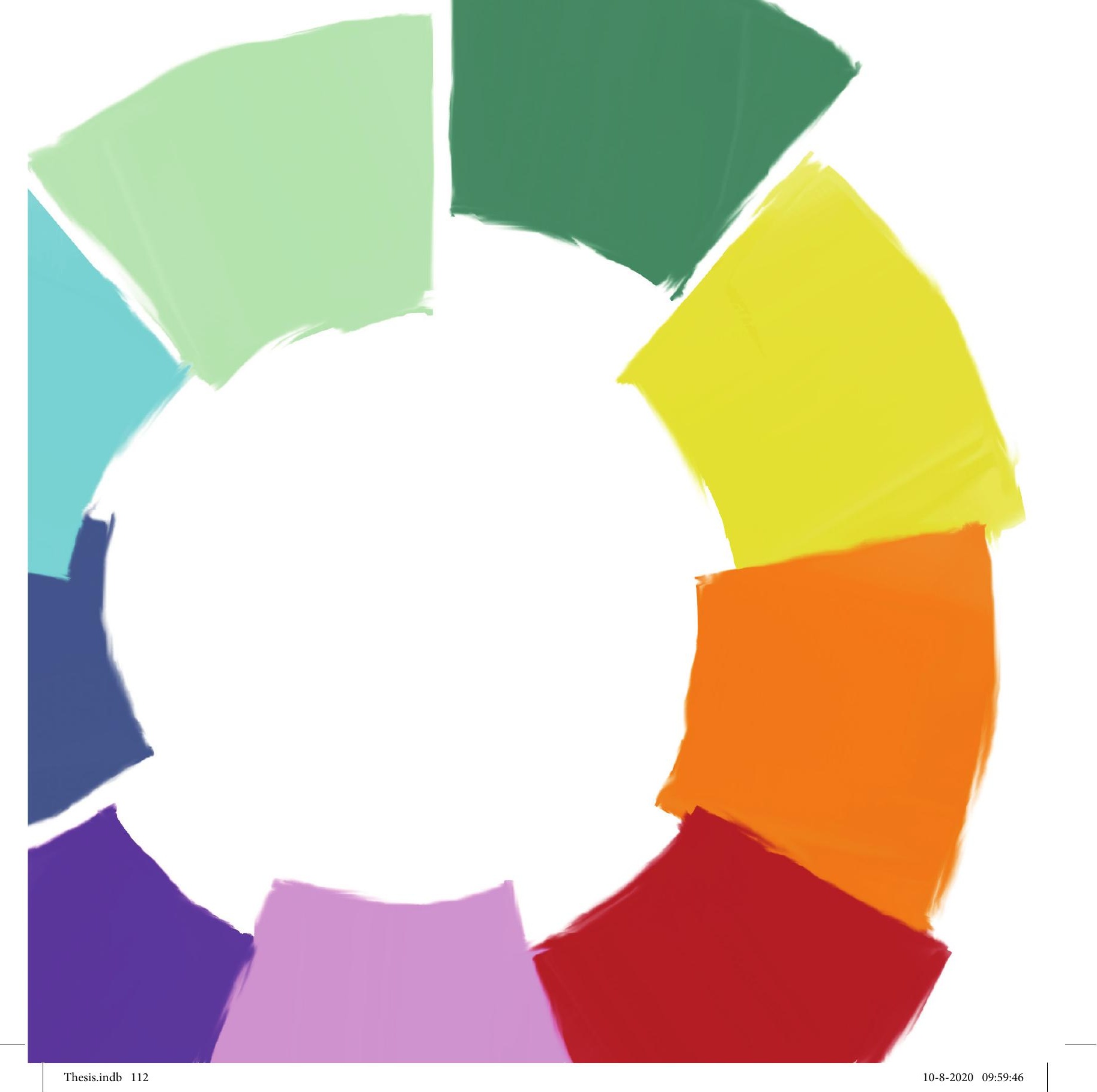




\section{Chapter 7 Development of a Versatile Microreactor Setup for Fluorescent Product Screening to Assess Activity of Single Catalyst Particles}

In this Chapter, we describe a droplet microfluidic setup based on fluorinated ethylene polymer (FEP) tubing to encapsulate and diagnose single catalyst particles, to demonstrate the feasibility of low-cost and easyto-fabricate devices. By coiling the tubing around a cylindrical heating device, this setup can be used to study reactions at elevated temperatures of around $100{ }^{\circ} \mathrm{C}$, using fluorescent read-out. With luminescence thermometry, the temperature deviations were determined to be max. $2.2{ }^{\circ} \mathrm{C}$. Droplets were stable and monodisperse in size at room temperature, but became instable above $100^{\circ} \mathrm{C}$. The fluorescence of in-situ synthesized benzocoumarin was used as a measure for the catalytic activity of multiple hydrotalcites at the start and end of the tubing. The residence times (and thus reaction times) for droplets in the tubing were 6.5 or $13 \mathrm{~min}$. After heating to $100{ }^{\circ} \mathrm{C}$, the fluorescence significantly increased for all hydrotalcites tested. Hydrotalcite loaded with $\mathrm{KOH}$ showed the highest catalytic activity. The major limitation for single catalyst particle diagnostics for hydrotalcites was the mechanical instability of the catalyst particles. This could in future work be overcome by using basic solid catalysts with a better mechanical stability, i.e. supported $\mathrm{MgO}$ or amino-functionalized silica. The developed microreactor is cheap, easy to fabricate, and highly versatile and the microchannel can be customized to different reagent phases or reaction times by tuning the properties or length of the FEP tubing

This work is based on the following manuscript: W. van den Bosch, An Approach Towards Single Catalyst Particle Diagnostics in Microfluidic Devices with Fluorescence Micro-Spectroscopy, Master Thesis. 


\subsection{Introduction}

In contrast to recent developments in microfluidic research towards more and more complicated lab-on-a-chip designs for advanced diagnostics, there are also research groups that focus their attention on the development of low-cost and easy-to-use microreactor systems. ${ }^{[1-3]}$ In the previous Chapters we have elaborated on the use of droplet microreactors for single particle diagnostics. External factors, such as local heating or gas permeation, were needed to start the catalytic reaction. Furthermore, magnetophoretic or dielectrophoretic sorting were implemented for sorting of Fluid Catalytic Cracking (FCC) particles. However, for a more facile screening of catalyst particles acting in liquidphase chemical reactions, easier reactor design should suffice.

In the general introduction of this $\mathrm{PhD}$ thesis, provided in Chapter 1, the co-flow configuration for droplet generation has been described. An easy way to assemble a coflow microreactor is by using capillary tubing of two sizes, ${ }^{[4,5]}$ and with commercially available connectors and T-assemblies, the use of difficult, clean-room fabricated microreactors becomes redundant (see Figure 7.2). In this Chapter, we describe the use of a simple tube-in-tube microreactor for the diagnostics of solid basic catalysts by monitoring the formation of fluorescent coumarins as product. The coumarin derivative that will be used as dye for our single particle diagnostics, needs to be synthesized in-situ with a one-step reaction. This one step reaction to induce fluorescence is important to be able to use the fluorescence intensity as a measure for catalytic activity.

Coumarins, an important family of heterocyclic compounds widely found in nature, as shown in Figure 7.1, exhibit interesting fluorescent properties that are highly sensitive to their local environment. ${ }^{[6]}$ These basic catalysts have been secribed in more detail in Section 1.3.3 in the General Introduction. They can be synthesized via condensation

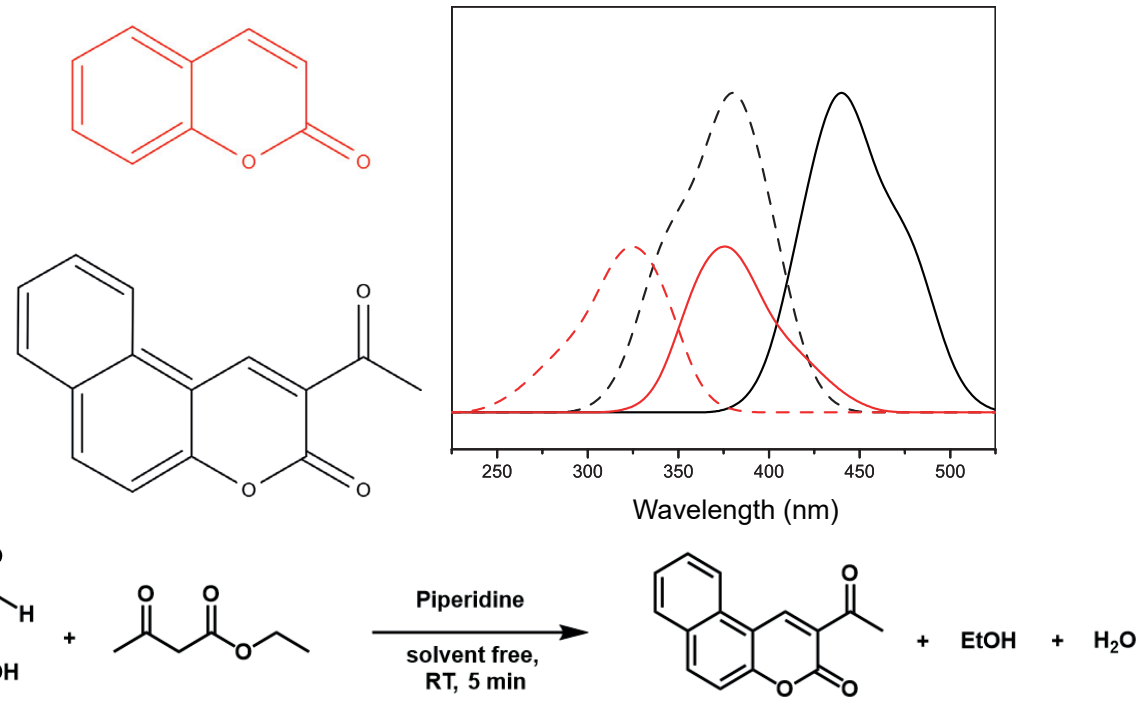

Figure 7.1: Molecular structure of normal coumarin (red) and extended benzocoumarin (black) and a schematic image of their absorption (dotted line) and emission (solid line) maxima. NB quantum yield is not displayed properly. The reaction scheme shows the Knoevenagel condensation to form benzocoumarin. 
reactions. ${ }^{[7,8]}$ For example, the Pechmann condensation is acid-catalyzed in organic solvents and requires high reaction temperatures and long reaction times, while the Knoevenagel condensation is base-catalyzed in more polar solvents and requires lower temperatures and shorter reaction times. Therefore, in this work we have opted for the Knoevenagel condensation to synthesize benzocoumarin, shown in Figure 7.1, in solventfree conditions at room temperature. ${ }^{[9]}$ In literature, piperidine has been used as efficient (homogeneous) catalyst for this Knoevenagel condensation. ${ }^{[9]}$ However, since we are working towards single catalyst particle diagnostics we are interested in a solid material that can catalyze this condensation reaction.

Solid catalysts that are known to be active towards the formation of coumarins in general, are hydrotalcites. Therefore, in this Chapter we have selected several hydrotalcite derivatives that could be of interest for the Knoevenagel condensation of our targeted benzocoumarin. As has been described in Chapter 1, hydrotalcites, or layered double hydroxides are $\mathrm{Mg}-\mathrm{Al}$ based solid basic materials that are composed of brucite layers with interlayer ions. ${ }^{[10]}$ When we encapsulate single hydrotalcite particles inside reactant droplets, it is possible to screen the activity of the catalyst particles. In this case, the continuous phase must be inert and cannot play a role in the catalytic reaction. For this setup, it is of great importance that the catalyst and the reactants come in contact with each other only right before the formation of droplets to be able to use the fluorescence signal of a droplet as a measure for the catalytic activity of the encapsulated catalyst particle. Therefore, an extra junction to mix these phases before the droplets are formed was added to the setup.

Droplets were created using silicon oil and a reactant mixture in an excess of ethyl acetoacetate as the continuous and dispersed phase respectively. A microreactor, shown in Figure 7.2, was constructed in a simple tube-in-tube fashion, using a co-flow configuration to create droplets.

Apart from the low production costs of this setup, one of the main advantages of using polymer tubing, is the ability to change the length of the microchannel. Next to

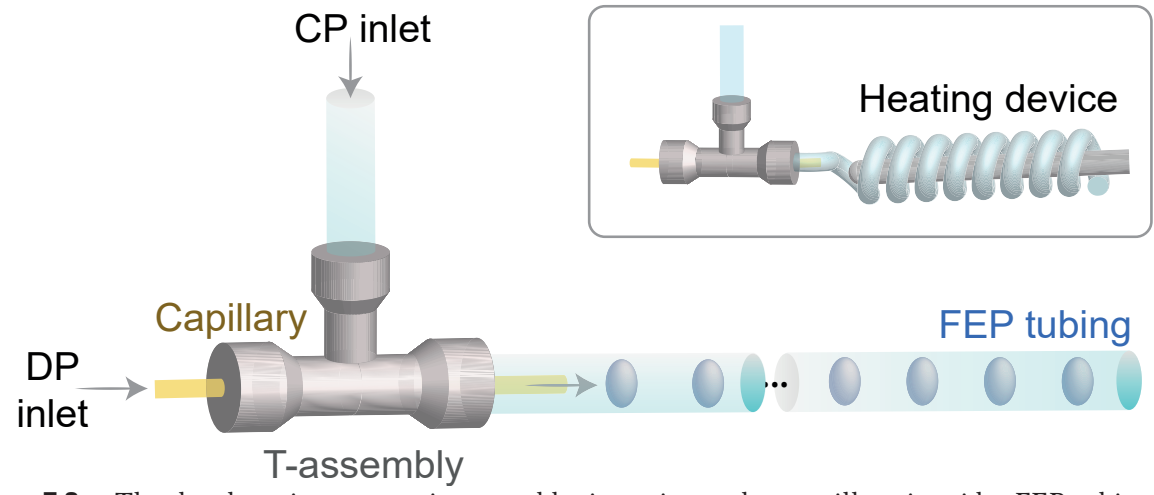

Figure 7.2: The droplet microreactor is created by inserting a glass capillary in wider FEP tubing, using a T-assembly piece. The dispersed phase (DP) is inserted via the capillary and the continuous phase (CP) flows from the side, around the capillary, in the FEP tubing. The inset shows the coiled tubing around a heating device. 
changing the flowrates, this adds a second tunable parameter to change the residence time of droplets in the microchannel. A possible disadvantage of this system is the relatively large scale of the microreactor. The final droplets have diameters close to the inner diameter of the tubing $(1 / 32$ ” or $\sim 800 \mu \mathrm{m})$. As a consequence, the identification of most catalyst particles becomes difficult due to their relatively small sizes. However, the characterization of a catalyst particle can in principle also be performed indirectly, by measuring the formation of products in the droplet instead of focusing on the particle itself. Furthermore, the ability to only heat parts of the tubing yields a big advantage for fluorescence measurements since it is known that most fluorophores, including benzocoumarins, have a lower fluorescence quantum yield at higher temperatures. ${ }^{[1]}$

In this Chapter, we will show the suitability of an easy-to-use microfluidics setup for single particle diagnostics, using the fluorescence of an in-situ synthesized benzocoumarin as a measure for catalytic activity. The accuracy of the heat transfer from the heating device to the droplets was tested with luminescence thermometry. Multiple hydrotalcite materials were tested as catalyst in solvent-free conditions. Furthermore, the reaction temperature was varied, and the reaction time was varied by adjusting the droplet flow rate. It will be shown that the mechanical stability of the catalyst particles is of utmost importance for single particle diagnostics. However, valuable results were obtained regarding the usability of the presented easy-to-use setup for droplet microfluidics.

\subsection{Experimental Section}

\subsubsection{Microfluidic Setup}

The microfluidic setup, as described in Figure 7.3, consists of a glass capillary (O.D.=360 $\mu \mathrm{m}, \mathrm{I} . \mathrm{D} .=150 \mu \mathrm{m})$ that is inserted in fluorinated ethylene polymer (FEP) tubing (O.D.=1/16", I.D.=1/32") that was coiled around a cylindrical heating device. A microreactor was assembled using two IDEX T-assembly pieces (P-712) with an inner diameter of 0.02 " $(0.5 \mathrm{~mm})$. One of the T-pieces was used to mix the reactants with the catalyst right before the formation of droplets. The second T-piece was used to create a co-flow configuration using fused silica tubing of $360 \mu \mathrm{m}$ O.D. inserted in FEP tubing (1/16” O.D., 1/32” I.D.).

Liquids were introduced using syringe pumps (NE-1000) equipped with $5 \mathrm{~mL}$ plastic syringes holding the different liquid phases. Catalyst particles were introduced by dispersing them in the reactants in the syringe via gentle stirring. Flowrates were varied between 15 and $30 \mu \mathrm{L} / \mathrm{min}$ total flow leading to total residence times between $\mathrm{X}_{1}$ and $\mathrm{X}_{2}$ of 13 and $6.5 \mathrm{~min}$, respectively. The total length of tubing between $\mathrm{X}_{1}$ and $\mathrm{X}_{2}$ was 40 $\mathrm{cm}$. For bulk experiments with piperidine, full conversion after 5 minutes is reported by Sugino et al. ${ }^{[9]}$ Bulk experiments with CHT (not shown) gave a threefold increase of fluorescence signal within $20 \mathrm{~min}$. By only heating part of the tubing and performing fluorescent read-out after the heating device where the tubing and droplets are back at room temperature, it is possible to compare the fluorescence signals at the start and end of the tubing. 


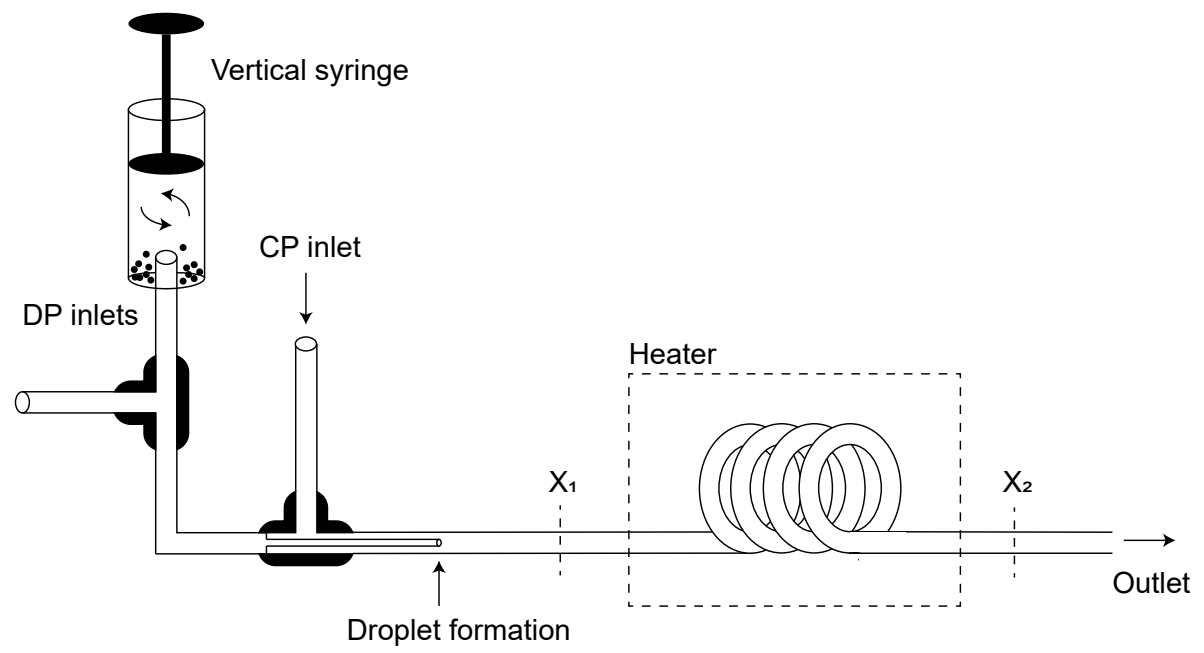

Figure 7.3: Schematic of the microreactor setup, illustrating how the tubing and T-assemblies are combined. To all inlets, syringes driven by syringe pumps are connected. The tube length between $\mathrm{X}_{1}$ and $\mathrm{X}_{2}$ is $40 \mathrm{~cm}$ The photos show the T-assembly for droplet generation (left) and the tubing coiled around the heater (right).

\subsubsection{Catalyst Preparation}

Multiple catalyst samples were prepared from a commercial hydrotalcite (Kisuma chemicals). Spherical hydrotalcite particles $(\mathrm{HT})$ were obtained by sieving $\mathrm{Mg}_{6} \mathrm{Al}_{2}(\mathrm{OH})_{16} \mathrm{CO}_{3} \cdot \mathrm{H}_{2} \mathrm{O}$ into a fraction of $75-90 \mu \mathrm{m}$. The particles were calcined at 450 ${ }^{\circ} \mathrm{C}$ for $6 \mathrm{~h}$ in air (ramp $1{ }^{\circ} \mathrm{C} / \mathrm{min}$ ) and named $\mathrm{CHT}$. Three impregnated samples were prepared from the calcined hydrotalcite material. To $30 \mathrm{mg}$ calcined hydrotalcites, 0.48 $\mathrm{mL}$ demi water, $\mathrm{KOH}$ solution $(0.125 \mathrm{M})$ or $\mathrm{KNO}_{3}$ solution $(0.125 \mathrm{M})$ were added. The wet impregnation was equilibrated for 24 hours before the mixture was dried for 24 $\mathrm{h}$ at $80^{\circ} \mathrm{C}$. This yielded five hydrotalcite-based catalyst materials in total: HT, CHT, $\mathrm{H}_{2} \mathrm{O} / \mathrm{CHT}, \mathrm{KOH} / \mathrm{CHT}$ and $\mathrm{KNO}_{3} / \mathrm{CHT}$. With a Bruker D2 phaser XRD diffractometer, measurements were performed to verify the crystal phase of the prepared samples.

\subsubsection{Benzocoumarin Formation and Detection}

A hydroxynaphtaldehyde ( $93 \%$, Acros Organics) solution in ethyl acetoacetate $(0.2 \mathrm{M}$, $99 \%$, Aldrich) was added to syringe DP1, the three different catalysts mentioned in the section before were dispersed in ethyl acetoacetate and added to syringe DP2 and silicon oil ( $\mathrm{Mw}=2000$, Alfa Aesar) was added to syringe CP. The flowrates for all three liquids were identical and set to 5 or $10 \mu \mathrm{L} / \mathrm{min}$. The reaction was performed at $20{ }^{\circ} \mathrm{C}$ and 100 ${ }^{\circ} \mathrm{C}$ and screened at position $\mathrm{X}_{1}$ and $\mathrm{X}_{2}$ with fluorescence microscopy. A Nikon Eclipse 90i confocal fluorescence microscope with a AlR scan head (10x objective) equipped with a $405 \mathrm{~nm}$ Melles Griot diode laser $(>50 \mathrm{~mW})$ was used to monitor the fluorescence intensity of individual droplets. Emission with a wavelength range 454.69-459.60 nm was used for measuring the averaged fluorescence with a framerate of $10 \mathrm{fps}$. For the measurements, first $\mathrm{X}_{1}$ was monitored, after which the field of view of the microscope was changed to $X_{2}$. Therefore, the droplets measured at $X_{1}$ do not necessarily match the droplets measured at $\mathrm{X}_{2}$. 


\subsubsection{Thermoluminescence Measurements Inside Tubing}

$\mathrm{NaYF}_{4}$ particles doped with $18 \% \mathrm{Yb}^{3+}$ and $2 \% \mathrm{Er}^{3+}$, which were synthesized as described earlier ${ }^{[12]}$ were used to measure the temperature inside the FEP tubing, using their thermoluminescence properties. A dispersion of these particles in cyclohexane was inserted in the microfluidic setup using a syringe. Two temperatures, namely 60 and 80 ${ }^{\circ} \mathrm{C}$, were used as setpoint for the heating device. The luminescence experiments were performed using an MDL-III laser of $500 \mathrm{~mW}$ with an excitation wavelength of $980 \mathrm{~nm}$. Upon excitation, light was collected via a fiber patch chord and a shortpass filter on an OceanOptics QEPro CCD.

\subsection{Results and Discussion}

\subsubsection{Validation of the Experimental Setup}

A low-cost droplet-microfluidic setup has been assembled using glass capillaries, FEP tubing and T-assemblies, as shown in Figure 7.3. The tubing was coiled around a cylindrical heating device. To verify the accuracy of the temperature setting and heat transfer from the heating device to the liquids in the tubing, luminescence thermometry was used to measure the temperature inside the tubing. For these measurements $\mathrm{NaYF}_{4}$ nanoparticles doped with $18 \% \mathrm{Yb}^{3+}$ and $2 \% \mathrm{Er}^{3+}$ were used as probes based on their temperature dependent luminescent properties. These nanoparticles have proven their usability as temperature probes in microfluidic systems, as described by Geitenbeek et al. ${ }^{[13]}$ The temperature was measured at seven setpoint temperatures and several positions in the tubing, resulting in a maximum deviation of $2.2^{\circ} \mathrm{C}$ from the set temperature, as shown in Table 7.1. These values are within the error margin determined for this sensing method. ${ }^{[12]}$ The relatively high $\mathrm{T}_{\text {MEASURED }}$ for room temperature (RT) is due to heating of the IR laser that is used for these experiments. In later experiments, RT is determined to be $20{ }^{\circ} \mathrm{C}$ and temperatures mentioned are set temperatures.

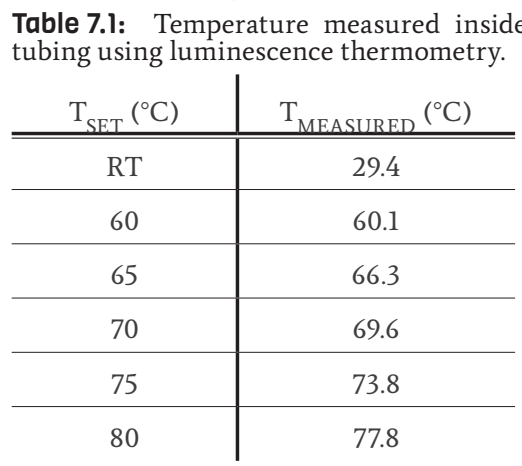

To validate the working principle of the developed setup, spherical HT particles were encapsulated in $0.7 \mu \mathrm{L}$ droplets. It is known that the density difference between solid particles and the liquid of the dispersed phase can cause fast precipitation of catalyst particles. This complicates the easy flow of particles through the microchannel. Once the particles are encapsulated, the droplet drags the particle through the microchannel, but before encapsulation, some measures were taken to prevent precipitation of particles at the walls of the tubing or syringe. Firstly, the content of the syringe was gently stirred. Secondly, the syringe was placed vertically to benefit from gravitational forces. Thirdly, a vibrational motor (mouser) was connected to the capillary through which the particles have to flow, to shake the tubing. Figure 7.4 provides a selection of frames of a collected movie, showing the droplets with encapsulated HT particles. Similar to previous Chapters, the encapsulation of catalyst particles is random because no precautions were 

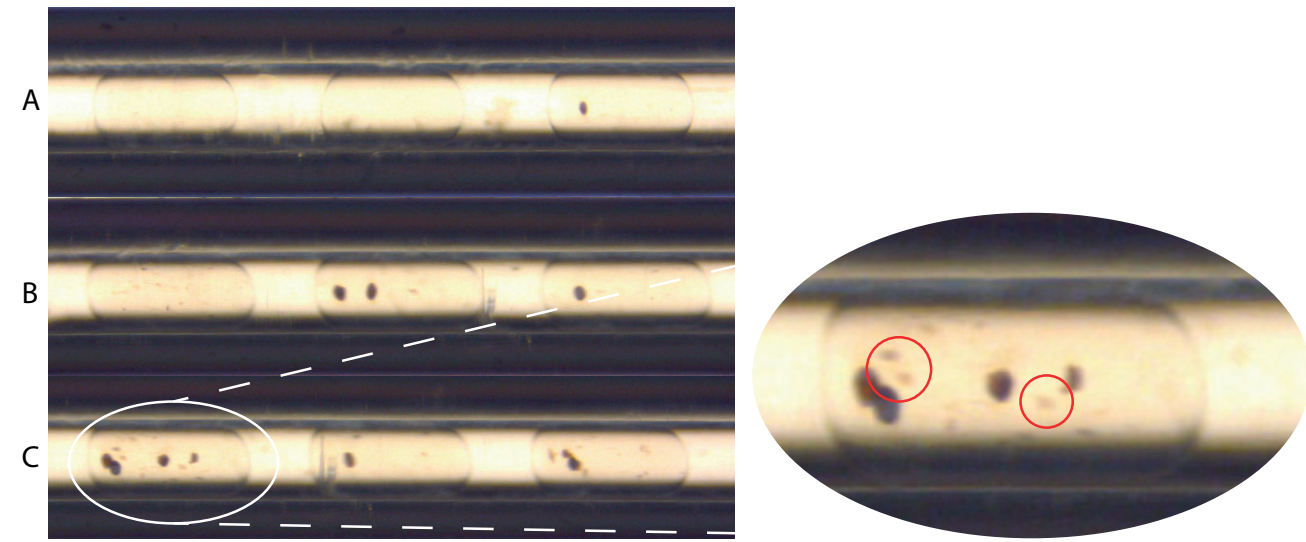

Figure 7.4: Images showing droplets of ethyl acetoacetate with encapsulated HT particles at different stirring rates. Gentle stirring (A) results in low amounts of droplets with single particles, with increasing stirring rate $(\mathrm{B}$ and $\mathrm{C})$ the number of particles per droplet increases. At the fastest stirring rate $(\mathrm{C})$, the particles break up in smaller pieces due to their low mechanical stability. The zoomed image shows these particle fragments indicated with red circles.

taken to align the particles before encapsulation. However, due to the lower mechanical stability of the HT particles compared to for example Fluid Catalytic Cracking (FCC) particles, more gentle stirring of the particles in the syringe is required, leading to a lower amount of encapsulated catalyst particles. At a faster stirring rate, the HT particles break down and form a homogeneously suspended grit in all droplets.

\subsubsection{Fluorescence Microscopy Measurements}

The droplets with encapsulated catalyst particles travel through the microchannel, where the catalytic reaction to form a fluorescent product can take place. The calcined (C)HT was tested for its catalytic activity towards the Knoevenagel condensation to produce the fluorescent organic molecule benzocoumarin (Figure 7.1). For these experiments, a dispersion of catalyst particles in ethyl acetoacetate coming from inlet $\mathrm{DP}_{1}$ was mixed at the first T-junction with a solution of hydroxynaphtaldehyde in ethyl acetoacetate coming from inlet $\mathrm{DP}_{2}$ to start the reaction. A few seconds later, droplets of the reaction mixture were formed in silicon oil as continuous phase. The droplets were screened at position $\mathrm{X}_{1}$ and $\mathrm{X}_{2}$ that were indicated in Figure 7.3 for 2 min. In Figure 7.5 , two images show a droplet at these two positions; the areas used for fluorescence analysis are indicated with red rectangles. The rectangles cover most of the width of the droplet, but do not include possible scattering or noise from the tubing wall. The collected data is then presented in the total counts averaged over all pixels. Each image consists of 1 frame, collected with a

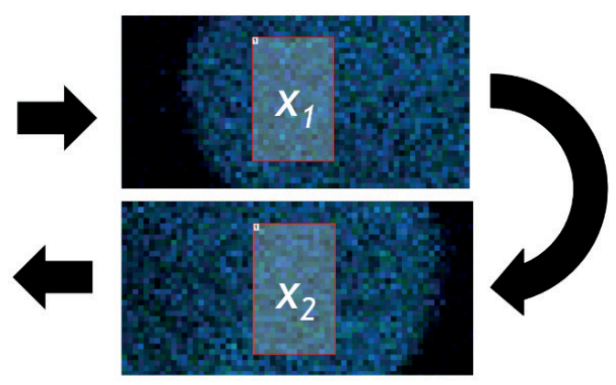

Figure 7.5: Fluorescence images of position $\mathrm{X}$ and $\mathrm{X}_{2}$ during the catalytic test with $\mathrm{CHT}$ at a total flowrate of $30 \mu \mathrm{L} / \mathrm{min}$ shows a minimal difference between the fluorescence intensities, indicating a lack of catalytic activity. The area within the rectangles of 360 pixels was used for analysis. 
framerate of $10 \mathrm{fps}$. A minimal difference in fluorescence and the absence of a visible catalyst particle indicate a low catalytic activity for this catalyst.

Figure 7.6 shows the fluorescence of $\mathrm{CHT}$ at these two different positions in the tubing, tested for both flowrates and two different temperatures, including a blank run without catalyst present. From Figure 7.6 it can be seen that the continuous silicon oil phase shows no fluorescence, indicating that the slightly fluorescent hydroxynaphtaldehyde or coumarin products do not diffuse out of the droplets. The blank run without catalyst shows a background fluorescence of approximately 150 counts for both positions.
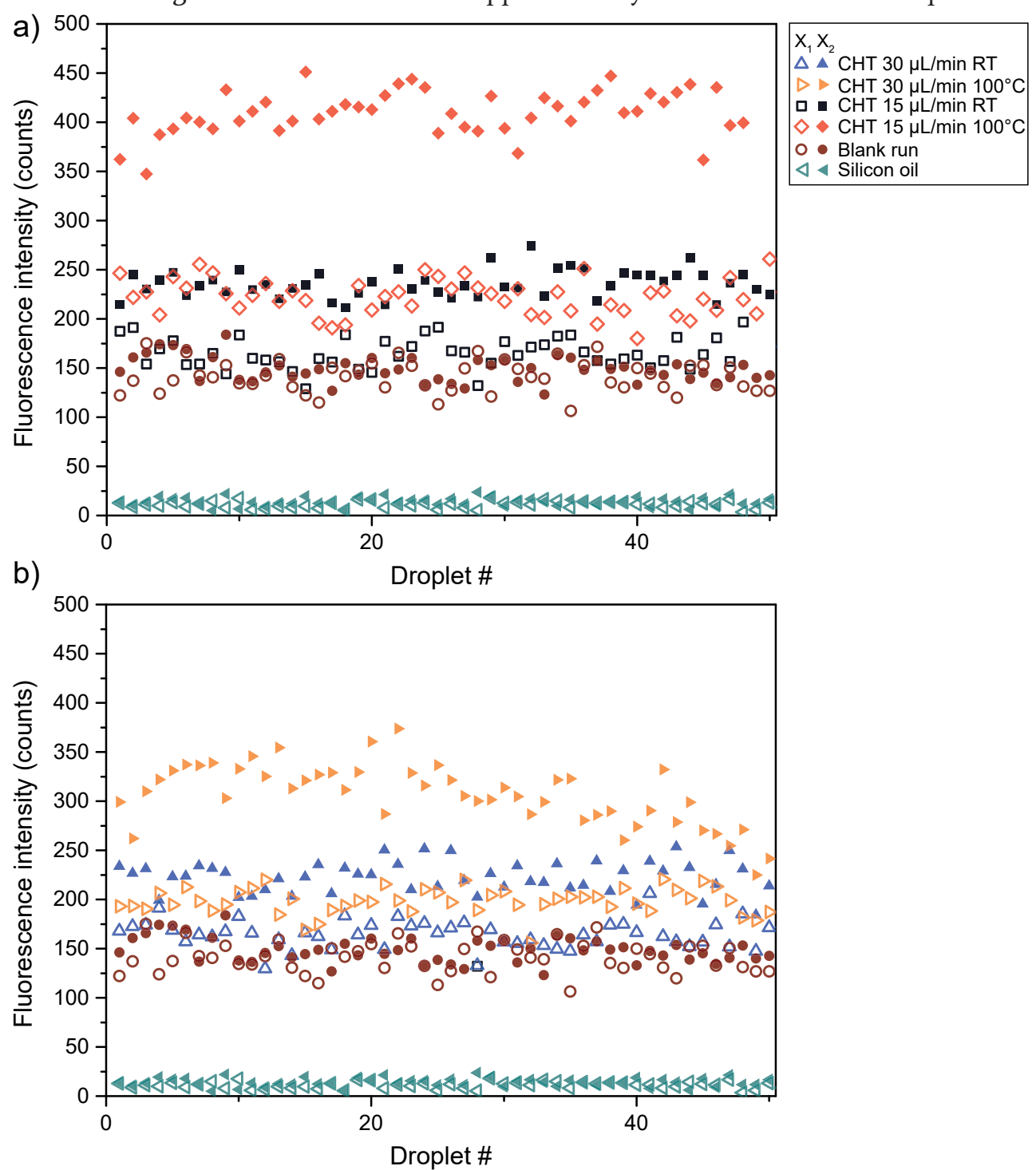

Figure 7.6: The fluorescence intensity upon excitation with $405 \mathrm{~nm}$ laser light of individual droplets is plotted for $15 \mu \mathrm{L} / \mathrm{min}$ (a) and $30 \mu \mathrm{L} / \mathrm{min}$ (b) at two different flowrates, including a blank run without catalyst and the fluorescence of the continuous phase silicon oil. 
This signal is most probably due to hydroxynaphtaldehyde. The signals at $\mathrm{X}_{2}$ for both measurements with $\mathrm{CHT}$ at room temperature are slightly elevated compared to the $\mathrm{X}_{1}$ position. Therefore, we conclude that the reaction is not proceeding at room temperature. At $100^{\circ} \mathrm{C}$ on the other hand, there is an increase in fluorescence visible. It is important to note that the stability of the droplets decreased at $100^{\circ} \mathrm{C}$, due to changes in the viscosity of both the continuous and dispersed phase. However, even though some droplets merged in between $\mathrm{X}_{1}$ and $\mathrm{X}_{2}$, this instability did not have an influence on the measurements due to the presence of homogeneously dispersed catalyst grit. The lower flowrate of $15 \mu \mathrm{L} /$ min total flow shows the highest fluorescence, due to the longer residence time between $\mathrm{X}_{1}$ and $\mathrm{X}_{2}$. The higher fluorescence is attributed to a higher benzocoumarin yield.

One important factor complicates all results: following Poisson statistics and microscope observations depicted in Figure 7.4 it is nearly impossible to encapsulate single particles in every droplet. For dilute particle concentrations it is expected that many droplets will be empty, and some droplets will contain multiple particles. This implicates that in some droplets (with a particle) the formation of the fluorescent benzocoumarin can take place, while in other empty droplets, it cannot. Therefore, it is not expected that the fluorescence intensity of all droplets is, within an error margin, similar. It is concluded that the constant concentration of grit present in all droplets due to the low mechanical stability of hydrotalcite particles, causes a constant catalytic activity in all droplets.

Further tests were performed to investigate the potential of the microfluidic setup for single particle diagnostics, for its use on other CHT derivatives. Two different CHT catalysts were prepared via impregnation of $\mathrm{KOH}$ and $\mathrm{KNO}_{3}$ in the interlayer area of the hydrotalcite structure. ${ }^{[14]}$ In Figure 7.7, the XRD patterns of all exchanged catalysts are shown. A first observation confirms that indeed all incorporated salts lead to the recovery of the crystal structure of the untreated HT. After calcination, the peaks for these planes are diminished due to the deformation of the crystal structure leading to a decrease in crystallinity; the exchange with $\mathrm{H}_{2} \mathrm{O}$ gives back the original HT. However,

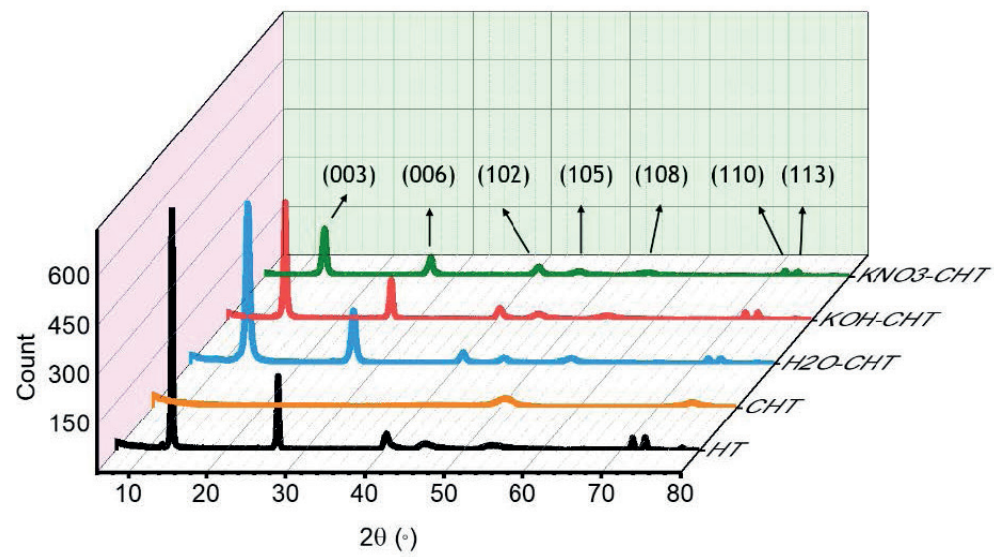

Figure 7.7: XRD patterns of the different synthesized hydrotalcite-based catalysts. The untreated hydrotalcite (HT) shows all peaks for the characteristic hydrotalcite planes: 003, 006, 102, 105,108, 110 and 113. The measurements were carried out with a Co K $\alpha$ radiation source with a step rate of $10^{\circ} \mathrm{min}$. 
it can be seen from the height of the peaks that the crystallinity of the materials after impregnation has decreased, due to the incorporation of defects. The ease of exchange of the monovalent ions decreases from $\mathrm{OH}^{-}$to $\mathrm{NO}_{3}{ }^{-[15]}$ In the corresponding XRD patterns a stronger decrease in the crystallinity of the $\mathrm{KNO}_{3} / \mathrm{CHT}$ versus $\mathrm{KOH} / \mathrm{CHT}$ is observed. We conclude from this that $\mathrm{KNO}_{3}$ has created more defects in the structure.

According to literature, the impregnation of HT with these basic salts leads to a similarly altered basicity of the catalyst. ${ }^{[16,17]}$ However, the loss in crystallinity will lead to less catalytic activity. In Figure 7.8, the results of testing several hydrotalcite derivatives are given. Devi et al. showed that salt-loaded hydrotalcites show higher activities towards Knoevenagel condensations at mild reaction conditions, with $\mathrm{KOH} / \mathrm{CHT}$ giving the highest conversion. ${ }^{[17]}$ Indeed for our reaction, $\mathrm{KOH} / \mathrm{CHT}$ gives a higher fluorescence compared to $\mathrm{KNO}_{3} / \mathrm{CHT}$, that itself is performing better than the normal $\mathrm{CHT}$ or uncalcined HT.

At the higher conversions, found for $\mathrm{KOH} / \mathrm{CHT}$, the hydrotalcite particles are also visible as bright spots in the droplets. This is attributed to the adsorption of fluorescent products to the surface of the catalyst particles. This enhanced fluorescence can be used in future experiments to locate a catalyst particle in the also fluorescent droplet, allowing for single particle diagnostics. Figure 7.9 shows such a fluorescent particle inside a droplet. The graph shows that the fluorescence intensity per particle varies strongly compared to the fluorescence of the liquid in the droplet.

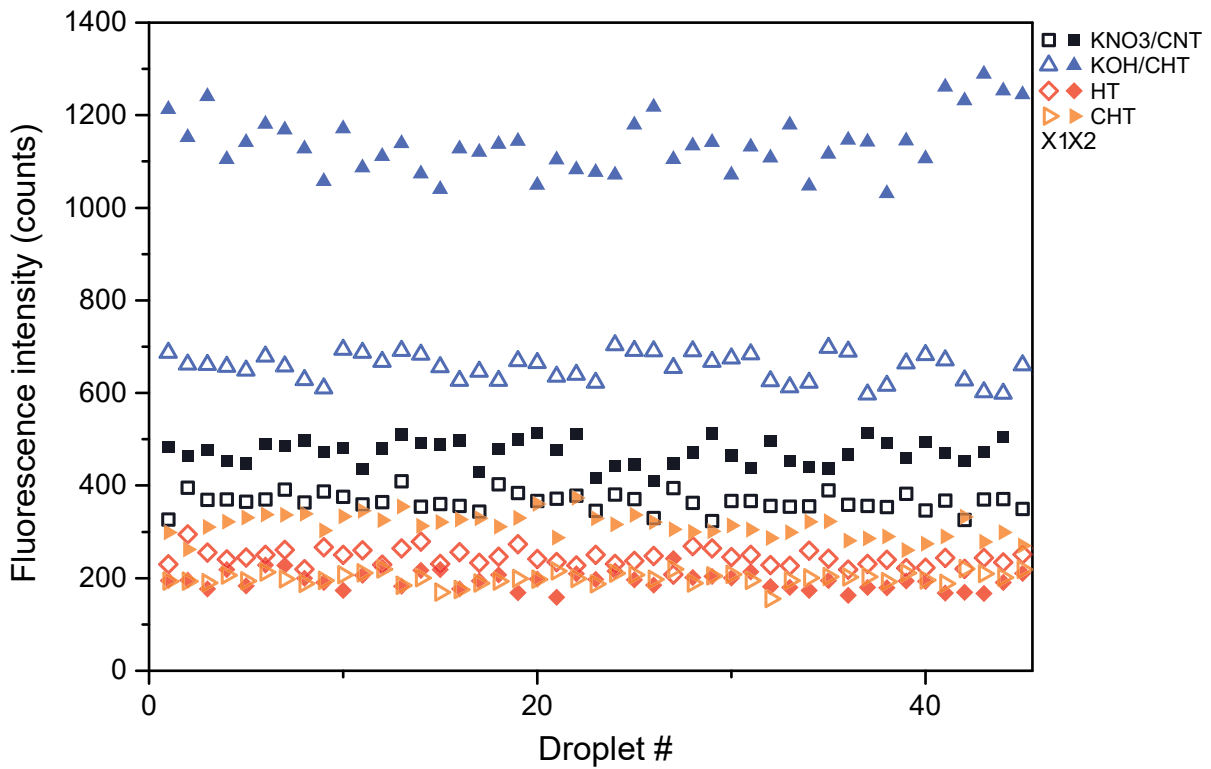

Figure 7.8: The fluorescence intensity upon excitation with $405 \mathrm{~nm}$ laser light of individual droplets is plotted for different hydrotalcite catalysts. 

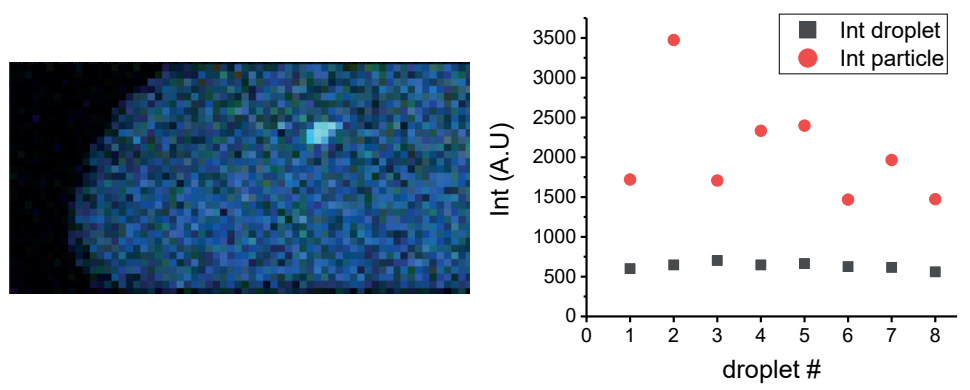

Figure 7.9: CFM image (top) of a droplet containing a fluorescent $\mathrm{KOH} / \mathrm{CHT}$ particle and a graph (bottom) showing the fluorescence intensity different between the fluorescent particles (red dots) and corresponding droplet (grey squares). The full height of the image corresponds to the inner diameter of the tubing: $\sim 800 \mu \mathrm{m}$.

\subsection{Conclusions}

By assembling fluorinated ethylene propylene (FEP) tubing and a glass capillary with a T-assembly, a simple tube-in-tube microreactor was developed for single catalyst particle diagnostics. The tubing was coiled around a cylindrical heating device and therefore suitable for reactions at elevated temperatures. With luminescence thermometry, the maximum temperature deviation from the set temperature was determined to be $2.2{ }^{\circ} \mathrm{C}$. The system was tested for the synthesis of a fluorescent benzocoumarin via a Knoevenagel condensation, catalyzed by several hydrotalcite derivatives. The droplets were stable and monodisperse in size at room temperature but became already less stable at $100{ }^{\circ} \mathrm{C}$. Successful encapsulation of hydrotalcite-based particles was achieved by stirring the catalyst dispersion in the syringe. However, no single particle diagnostics could be performed as the particles were mechanically instable and therefore a constant concentration of smaller particles was always present in the droplets. Fluorescence intensity measurements were carried out to screen the performance of different hydrotalcite catalysts for the synthesis of the fluorescent coumarin. The fluorescence intensity was screened at two positions on the microchannel; one before and one after the heating device. It was found that the reaction is not (fully) proceeding at room temperature. After heating to $100^{\circ} \mathrm{C}$, the fluorescence significantly increases for all catalysts tested. A KOHloaded hydrotalcite showed the most significant catalytic activity. Lowering the flowrate results in more conversion due to longer residence times in the microchannel.

By changing the catalyst to one with a higher stability, i.e. supported $\mathrm{MgO}$ or amino functionalized silica, this easy-to-use microfluidic setup shows great promise for the diagnostics of single catalyst particles. These catalysts contain strong enough basic sites that can deprotonate ethyl acetoacetate to facilitate the Knoevenagel condensation reaction. Due to its cheap and easy fabrication, the microchannel can be customized to the different reagent phases or reaction times needed by increasing the amount of T-assemblies used or elongating the FEP tubing.

\subsection{Acknowledgements}

We thank Carolien Vis (Utrecht University, UU) for the discussions regarding Knoevenagel condensation chemistry and the microfluidic setup and Thomas van 


\section{Swieten (UU) for luminescence measurements.}

\subsection{References}

[1] J. M. Zhang, A. A. Aguirre-Pablo, E. Q. Li, U. Buttner, S. T. Thoroddsen, RSC Adv. 2016, 6, 81120-81129.

[2] V. Faustino, S. O. Catarino, R. Lima, G. Minas, J. Biomech. 2016, 49, 2280-2292.

[3] C. W. Tsao, Micromachines 2016, 7, 225.

[4] S. K. Luther, S. Will, A. Braeuer, Lab Chip 2014, 14, 2910-2913.

[5] A. S. Utada, E. Lorenceau, D. R. Link, P. D. Kaplan, H. A. Stone, D. A. Weitz, Science (80-. ). 2005, 308, 537-541.

[6] B. D. Wagner, Molecules 2009, 14, 210-237.

[7] R. H. Vekariya, H. D. Patel, Synth. Commun. 2014, 44, 2756-2788.

[8] A. S. Zambare, F. A. Kalam Khan, S. P. Zambare, S. D. Shinde, J. N. Sangshetti, Curr. Org. Chem. 2015, 20, 798-828.

[9] T. Sugino, K. Tanaka, Chem. Lett. 2002, 30, 110-111.

[10] W. Bing, M. Wei, J. Solid State Chem. 2019, 269, 184-194.

[11] M. Brandt, "Quenching processes," in Introduction to Spectroscopy, 2010, pp. 31-37.

[12] R. G. Geitenbeek, P. T. Prins, W. Albrecht, A. Van Blaaderen, B. M. Weckhuysen, A. Meijerink, J. Phys. Chem. C 2017, 121, 3503-3510.

[13] R. G. Geitenbeek, J. C. Vollenbroek, H. M. H. Weijgertze, C. B. M. Tregouet, A.-E. Nieuwelink, C. L. Kennedy, B. M. Weckhhuysen, D. Lohse, A. van Blaaderen, A. van den Berg, M. Odijk, A. Meijerink, Lab Chip 2019, 19, 1236-1246.

[14] S. Miyata, Clays Clay Miner. 1983, 31, 305-311.

[15] S. P. Newman, W. Jones, New J. Chem. 1998, 22, 105-115.

[16] J. Zhao, J. Xie, C. T. Au, S. F. Yin, Appl. Catal. A Gen. 2013, 467, 33-37.

[17] R. Devi, P. Begum, P. Bharali, R. C. Deka, ACS Omega 2018, 3, 7086-7095. 
Development of a Versatile Microreactor Setup for Fluorescent Product Screening to Assess Activity of Single Catalyst Particles 


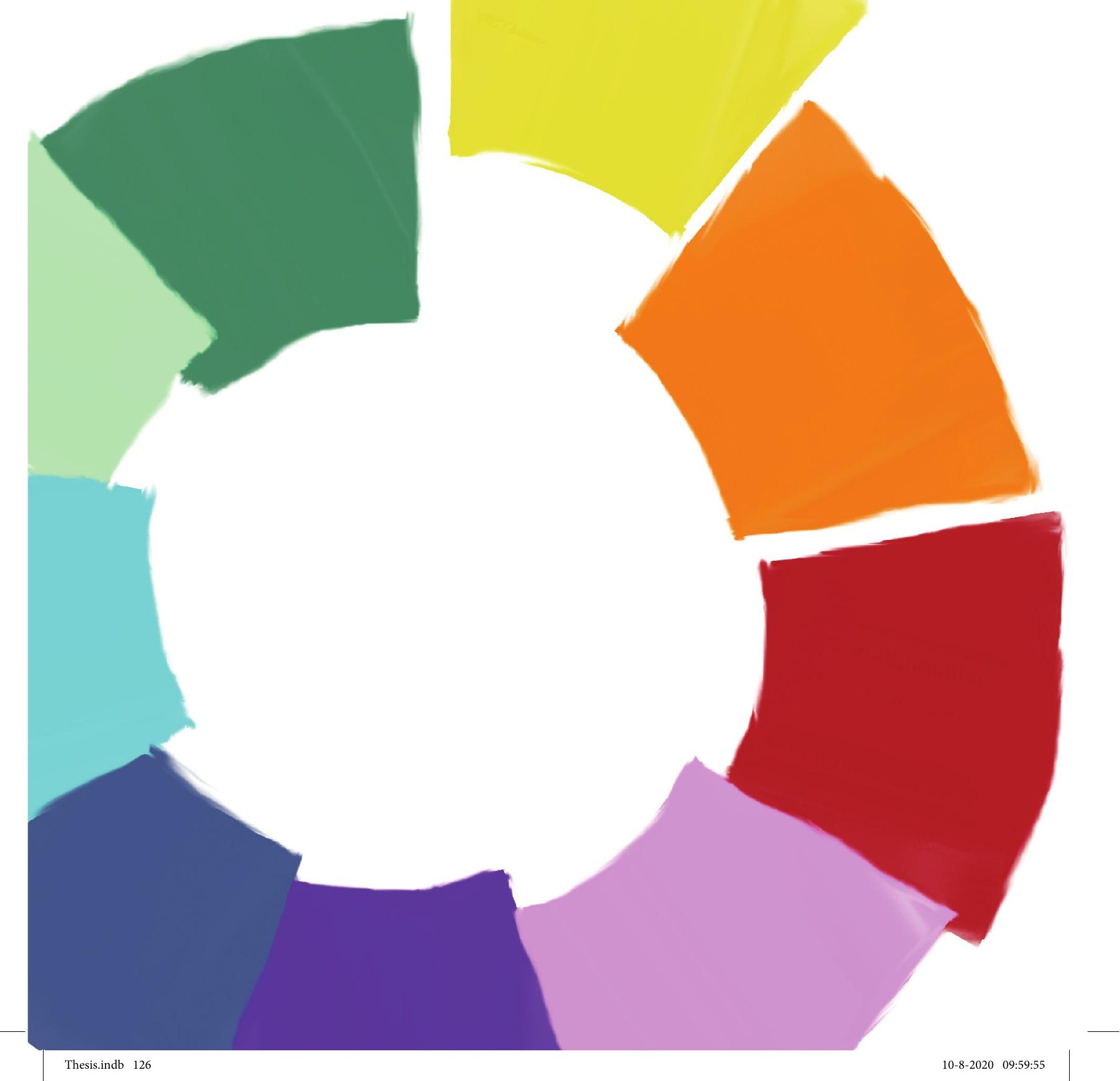




\section{Chapter 8 Summary, Concluding Remarks and Outlook}




\subsection{Summary}

In this $\mathrm{PhD}$ thesis, research on the use of microfluidics and spectroscopy for single catalyst particle diagnostics has been developed and described.

In Chapter 2, a newly designed and constructed 3-D printed microfluidic chip has been used to magnetophoretically sort a set of fluid catalytic cracking (FCC) particles into five different fractions. Each of these fractions could be analyzed in great detail, which allowed to relate their chemical composition to their magnetic moment. The increase in magnetic moment originates from the presence of metal poisons, in particularly Fe. The magnetic observations strongly point into the direction that the variation in magnetic moment is caused by an increased number, different form but similar size of Fe-containing nanoclusters. The spread within acidity of each fraction is still large: not all highly magnetic FCC particles show a high degree of deactivation. We believe that this technology could be used to sort other magnetic catalysts for analysis purposes, for example Fe-based Fischer-Tropsch synthesis catalysts. It can also serve as a practical tool to decrease the heterogeneity of the to be analyzed samples from an industrial reactor unit, thereby providing an alternative method for catalyst quality control measurements.

Chapter 3 shows that correlating information from multiple characterization techniques per FCC catalyst particle, provides valuable insights in the heterogeneity of density-separated FCC particles. Furthermore, the possibility to measure multiple catalyst particles adds statistical value to the data obtained. Where bulk characterization measurements such as chemical analysis by inductively coupled plasma-optical emission spectroscopy (ICP-OES), gas sorption and ammonia-temperature programmed desorption (TPD) can give insight in the trends between the six density-separated ECAT fractions, the diagnostics of single catalyst particles can show acidity and accessibility trends even within each fraction, as provided by catalyst staining with dye molecules in combination with fluorescence microscopy. Furthermore, the large intrinsic interparticle heterogeneity of each density separated fraction was visualized by overlaying micro-X-ray fluorescence ( $\mu \mathrm{XRF}$ ) maps of $\mathrm{Fe}$ and $\mathrm{Ni}$ with the fluorescence microscopy maps, obtained after catalyst particle staining. By using this approach, the degree of deactivation could be linked to the Ni content on a single catalyst particle level, in a sense that FCC particles with a high Ni content do not show high acidity.

In Chapters 4 and 5, two droplet-based microreactors were described that can be used for chemical analysis at elevated temperatures and pressure. Stable formation of monodisperse droplets, as well as heating of the droplets up to $120^{\circ} \mathrm{C}$ and up to pressures of $7 \mathrm{bar}$, has been achieved with the droplet microreactor in Chapter 4 . The temperature of the heater elements can be controlled at a standard deviation of approximately $2.6^{\circ} \mathrm{C}$ from the setpoint. This microreactor was used for high-throughput screening of individual FCC catalyst particles, based on their activity. The screening was performed in-situ at elevated temperatures up to $95{ }^{\circ} \mathrm{C}$ using the oligomerization of 4-methoxystyrene as a marker for the catalysts' activity. In total $\sim 1000$ FCC catalyst particles were detected at an average rate of 1 particle per 2.4 seconds. The microreactor set-up described in Chapter 
5 also has an implemented sorting system using dielectrophoresis (DEP). FCC particles stained with 4-fluorostyrene were sorted based on their fluorescence intensity, and thus their Brønsted acidity. After sorting and retrieving the FCC particles, they were analyzed using fluorescence microscopy and micro X-ray fluorescence ( $\mu \mathrm{XRF}$ ) in the same manner as was described in Chapter 3. This post-sorting characterization showed that the nonsorted FCC particles contain higher Ni levels than the sorted FCC particles. Since Ni is not present in fresh FCC particles and can only be accumulated by spending time in an industrial reactor, this indicates that the non-sorted FCC particles are the more aged particles.

Chapters 6 and 7 are dedicated to other catalytic systems. In Chapter 6, we show the proof of concept of a polydimethylsiloxane (PDMS) microreactor for high-throughput, multiphase catalytic reactions, demonstrating the liquid-phase hydrogenation of methylene blue $(\mathrm{MB})$. A liquid channel with $\mathrm{Pd} / \mathrm{SiO}_{2}$ particles of $40 \mu \mathrm{m}$ encapsulated in droplets containing $20 \mathrm{ppm} \mathrm{MB}$ in ethanol are flanked by two gas channels with $\mathrm{H}_{2}$, separated with $50 \mu \mathrm{m}$ PDMS walls. Due to the high permeability of PDMS, $\mathrm{H}_{2}$ diffuses into the liquid channels and facilitates the Pd-catalyzed hydrogenation of $\mathrm{MB}$ at room temperature. Chapter 7 describes a simple fluorinated ethylene propylene (FEP)-based tube-in-tube microreactor for single catalyst particle diagnostics. The system was tested for the synthesis of a fluorescent benzocoumarin via a Knoevenagel condensation, catalyzed by several hydrotalcite derivatives. Microdroplets of $0.7 \mu \mathrm{L}$ were formed by mixing a dispersion of spherical hydrotalcite-based catalyst particles in ethyl acetoacetate with a hydroxynaphtaldehyde in ethyl acetoacetate solution at a T-junction. In the next step, droplets of the combined reactant and catalyst phase were created in a continuous phase of silicon oil at a co-axial junction. Due to the mechanical instability of the catalyst particles, no single particle diagnostics could be performed. However, fluorescence intensity measurements were carried out to screen the performance of four different hydrotalcite catalysts for the synthesis of the fluorescent coumarin.

\subsection{Concluding remarks}

In this $\mathrm{PhD}$ thesis, different (microfluidic) setups are described to characterize single catalyst particles. The techniques that were focused on FCC particles, as described in Chapters 2-5, show different approaches to analyze their degree of deactivation via metal (i.e., Fe and $\mathrm{Ni}$ ) accumulation, acidity and accessibility changes and sort the catalyst particles accordingly. By combining these sorting and analysis techniques a diagnostics platform is obtained that can select the desired catalyst particles from a sample. Especially interesting will be the selection of the most active ones and determine why they have maintained their catalytic performance. In principle, this could lead to new design rules to make more active and/or stable FCC catalyst materials for various feedstocks.

The final two chapters of this $\mathrm{PhD}$ thesis show the great versatility of microfluidics for the evaluation of different catalyst systems, such as supported metal catalysts or hydrotalcites. Extra advances in separation and analytical devices will even increase their usability further. For example, by placing the PDMS chip from Chapter 6 on a 
heating stage, reactions at elevated temperatures can be performed. Due to the thermal stability of crosslinked PDMS, it can be heated to $400{ }^{\circ} \mathrm{C}$ before it starts to degrade. However, in this situation more problems arise, such as the boiling of solvents or a non-uniform heating of the microreactor. In addition, the optical properties of PDMS allow for monitoring chemical reactions with visible (laser) light, therefore allowing to perform in-line UV-vis or Raman spectroscopy. These spectroscopic techniques should be optimized in a way that they can work fast and can detect individual droplets at high flow rates. More sophisticated microreactor designs with microfluidic droplet sorters can then be implemented to sort the catalyst particles of interest.

Furthermore, the easy-to-use microfluidic setup from Chapter 7 shows great promise for the diagnostics of single catalyst particles. Due to its cheap and easy fabrication, the microchannel can be customized to the different reagent phases or reaction times needed by increasing the amount of T-assemblies used or elongating the FEP tubing, respectively. In this case, the to be sorted catalyst should have a higher mechanical stability compared to the hydrotalcites used in Chapter 7.

\subsection{Outlook}

To further illustrate the versatility of droplet-based microfluidics for catalysis and to showcase the compatibility with Raman spectroscopy, the tube-in-tube reactor that has been discussed in Chapter 7, was used to prepare a Pickering emulsion for biphasic catalysis. Pickering emulsions (PEs) are stabilized by the adsorption of amphiphilic solid particles onto the liquid-liquid interface, creating either water-in-oil or oil-in-water emulsions depending on the affinity of the particles (hydrophilic or hydrophobic) for one of the two phases. ${ }^{[1,2]}$ With surfactant-stabilized emulsions it is difficult to recover the surfactants and separate the organic and aqueous phase, but with PEs this can be easily done by, for example, centrifugation, which makes them suitable reversible reaction media. Recently, interest has been shown in using PEs as reaction media for biphasic or tandem catalysis. ${ }^{[3-7]}$

Here we use the tube-in-tube co-flow microfluidic setup, shown in Figure 8.1, to make water-in-oil droplets with and without the use of stabilizing particles, to prepare in-flow PEs. The PE was used as reaction medium to perform the biphasic catalytic conversion of benzaldehyde dimethyl acetal to benzaldehyde, which can be easily followed by insitu Raman spectroscopy. The formation of product was followed over time at various temperatures. This reaction is used as an acid catalyzed probe reaction of which we have shown the benefit of using a PE over a conventional biphasic system before. ${ }^{[6]}$ One of the advantages of using droplet-based microfluidics to prepare a $\mathrm{PE}$, is the easy way to gain control over the droplet size, leading to a more homogeneous PE system. ${ }^{[8]}$ In contrast to a bulk biphasic system, inside the tubing of the microfluidic setup, the droplets can exist without the use of surfactants. However, to give droplets in a microchannel extra stability, often surfactants or, to a lesser extent, particles are added. This stability is needed to prevent coalescence when droplets collide with each other. Furthermore, by using chemically resistant and transparent polymer tubing, the formation and stability 


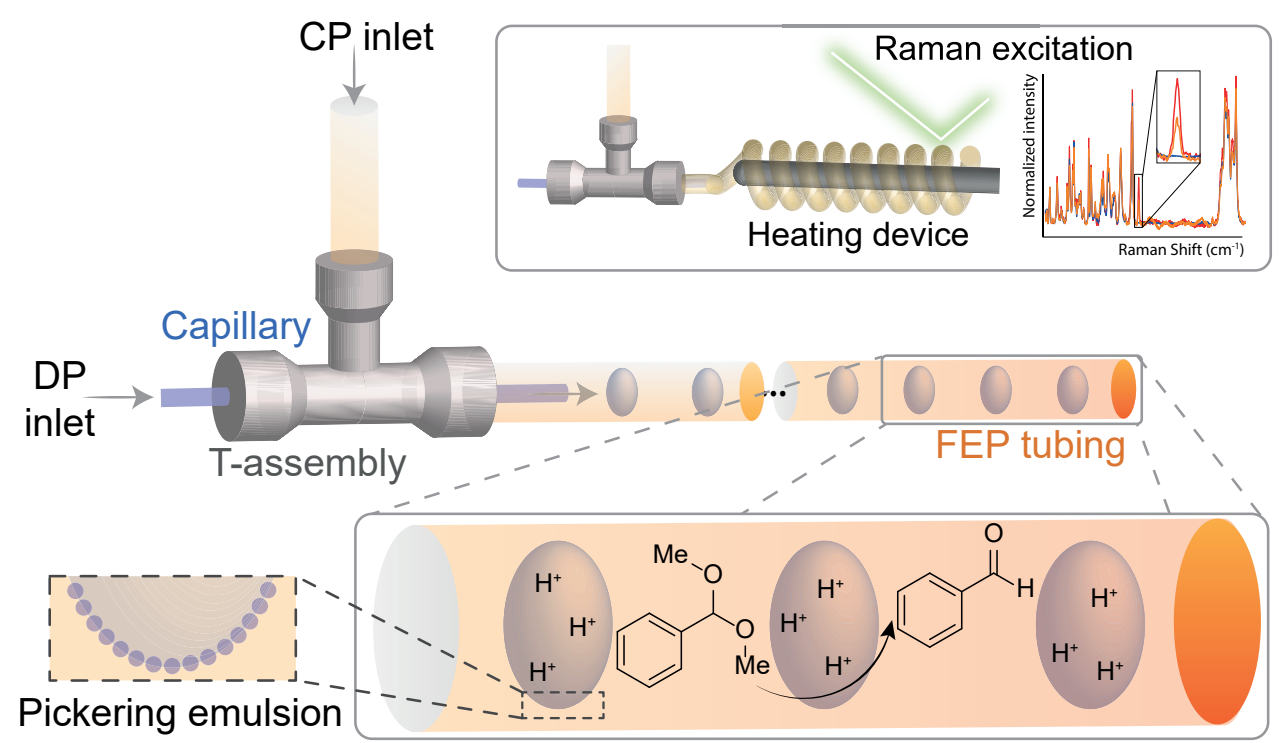

Figure 8.1: Top: droplet microfluidic approach for the tandem catalytic deacetalization-Knoevenagel condensation reaction. The deacetalization reaction can be monitored in-situ using Raman spectroscopy. Zoom: tube-in-tube co-flow configuration. Bottom: the acid-catalyzed deacetalization reaction of benzaldehyde dimethyl acetal to benzaldehyde, inside the FEP tubing.

of the droplets is clearly visible during the complete residence time in the tubing. Also, this transparency allows for in-situ spectroscopy: by measuring at different positions in the flow channel, different reaction times can be monitored. The reaction time can be easily controlled by either changing the total flow rate or by changing the length of the tubing. Also, one of the advantages of in-flow PEs over bulk PEs is the automatic phase separation after the tubing's outlet. ${ }^{[9]}$ This makes a post reaction analysis of both phases relatively easy.

Droplets were created by a tube-in-tube co-flow microfluidic setup using hydrophobic outer tubing (FEP) and hydrophilic inner tubing (fused silica). Due to this system configuration, w/o droplets with a homogeneous size distribution were formed. The droplets were spherical and their size was similar to the inner diameter of the tubing. To obtain droplets with a proper spacing, that means the space between two droplets equals the diameter of one droplet, the flow rates of continuous organic and dispersed aqueous phase were $20 \mu \mathrm{L} / \mathrm{min}$ and $5 \mu \mathrm{L} / \mathrm{min}$ respectively. This results in an optimal droplet size and catalyst to reagent mol fraction. As the total length of the tubing was $150 \mathrm{~cm}$, with an inner diameter of $0.76 \mathrm{~mm}$, at a total flow rate of $25 \mu \mathrm{L} / \mathrm{min}$ the total residence time inside the tubing and therefore reaction time, was calculated to be $27 \mathrm{~min}$. However, contact between two droplets could occur over the length of the tubing, leading to coalescence in the absence of stabilizing particles. In the experiments with stabilizing particles present these droplets remained stable and no coalescence was observed.

The deacetalization experiments were performed with benzaldehyde dimethyl acetal in 4-propylguaiacol as the continuous phase and water containing an acid catalyst as the dispersed phase. To investigate the difference in performance of PEs and biphasic 
systems (BS) the experiment was carried out with and without the use of stabilizing particles. The formation of benzaldehyde was followed using in-situ Raman spectroscopy, by collecting averaged spectra including multiple droplets, using $532 \mathrm{~nm}$ as excitation wavelength. The performance of the PEs and BS for the deacetalization reaction was determined at different temperatures. This reaction produces solely benzaldehyde, which shows a very distinct peak from the $\mathrm{C}=\mathrm{O}$ stretch vibration at $1700 \mathrm{~cm}^{-1}$ in the Raman spectrum. To monitor the benzaldehyde concentration, a calibration of the benzaldehyde signal was performed using $0.5 \mathrm{M}$ carbon tetrachloride $\left(\mathrm{CCl}_{4}\right)$ as internal standard with benzaldehyde concentrations between 0 and $1 \mathrm{M}$. After normalization of all spectra on the peak of the symmetrical stretch vibration of $\mathrm{CCl}_{4}\left(460 \mathrm{~cm}^{-1}\right)$, the height of the benzaldehyde peak $\left(1700 \mathrm{~cm}^{-1}\right)$ was used for quantification. Raman peaks at 460 and 1700 $\mathrm{cm}^{-1}$ and the resulting calibration curve, with a linear response over the concentration range, are shown in Figure 8.2.
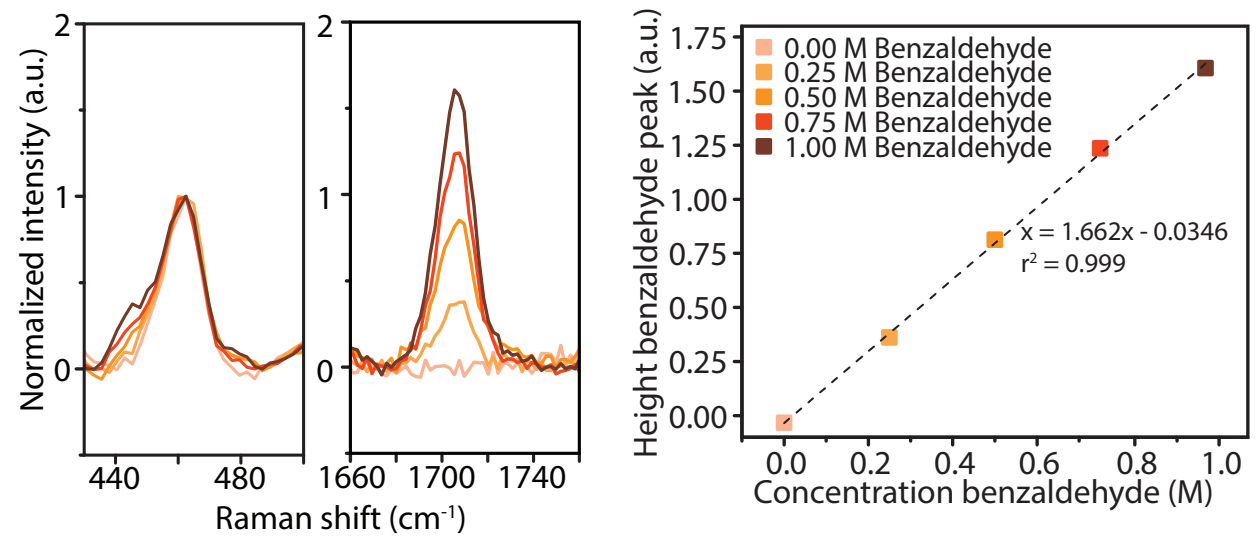

Figure 8.2: Calibration of benzaldehyde in Raman spectroscopy using $0.5 \mathrm{M} \mathrm{CCl}_{4}$ as internal standard, left: Raman spectra of the $\mathrm{CCl}_{4}$ peak at $460 \mathrm{~cm}^{-1}$ and benzaldehyde peak at $1700 \mathrm{~cm}^{-1}$, right: calibration curve based on benzaldehyde peak at $1700 \mathrm{~cm}^{-1}$.

The deacetalization reaction was performed at room temperature, $60^{\circ} \mathrm{C}$ and $90^{\circ} \mathrm{C}$, and with and without addition of silica. Although the addition of silica to form the PE leads to an increase in viscosity, this did not change the droplet size significantly. The presence of silica in the reaction mixture did not influence the Raman measurements either. The spectra were taken at different positions in the tubing (Figure 8.3), with a reaction time of approximately 2 min per loop. At loop 0 , which is almost directly after the formation

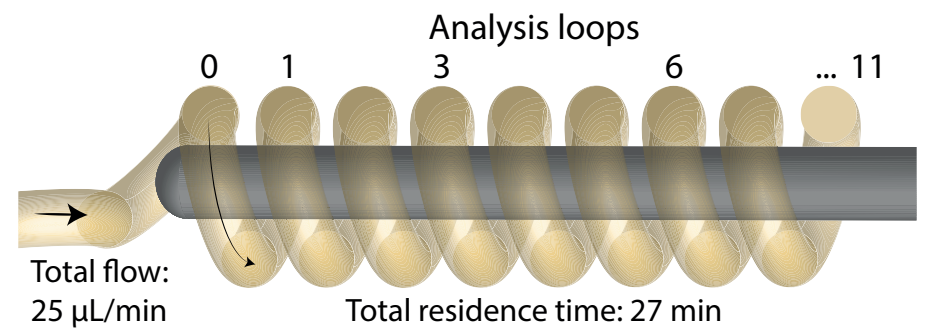

Figure 8.3: Schematic drawing of in-situ Raman spectroscopy analysis loops. Every loop is approximately 2 minutes residence time. At the position of loop 0 , droplets have just been generated. 
of droplets, the reaction time is $5 \mathrm{~s}$. To avoid any influences of acid staining on the FEP tubing, the system was flushed with $\mathrm{NaOH}$ solution between every measurement.

The height of the peak corresponding to the $\mathrm{C}=\mathrm{O}$ stretch vibration at $\sim 1700 \mathrm{~cm}^{-1}$ was used to determine the concentration of benzaldehyde over time at each loop, as shown in Figure 8.4. The kinetic profiles of the benzaldehyde formation at all temperatures and with and without addition of silica are shown in Figure 8.5. At room temperature without any silica present, the reaction proceeds very slowly and after $22 \mathrm{~min}$ a benzaldehyde yield of approximately $10 \%$ was observed. Increasing the reaction temperature from RT to $60{ }^{\circ} \mathrm{C}$ led to a significant increase in reaction rate, with full conversion reached after approximately $18 \mathrm{~min}$. At $90^{\circ} \mathrm{C}$, the reaction proceeds even faster with full conversion reached after $2 \mathrm{~min}$. Addition of silica to the reaction mixture led to even faster reactions,
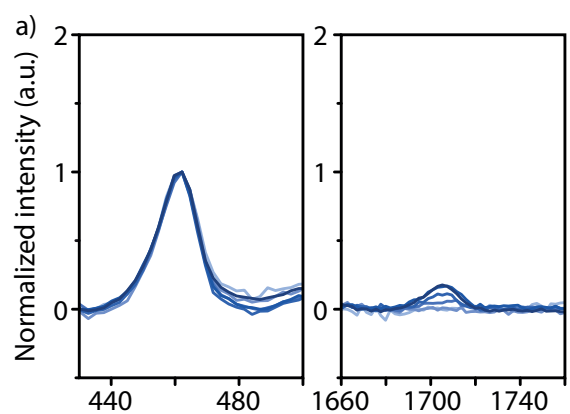

b)
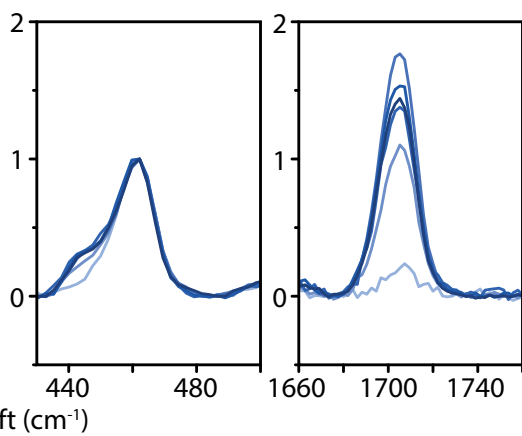

d) 2
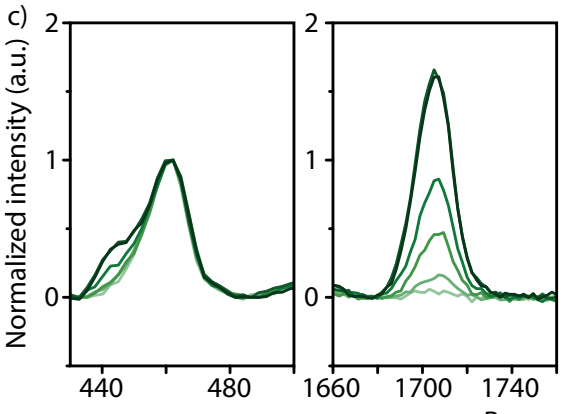

Raman shif
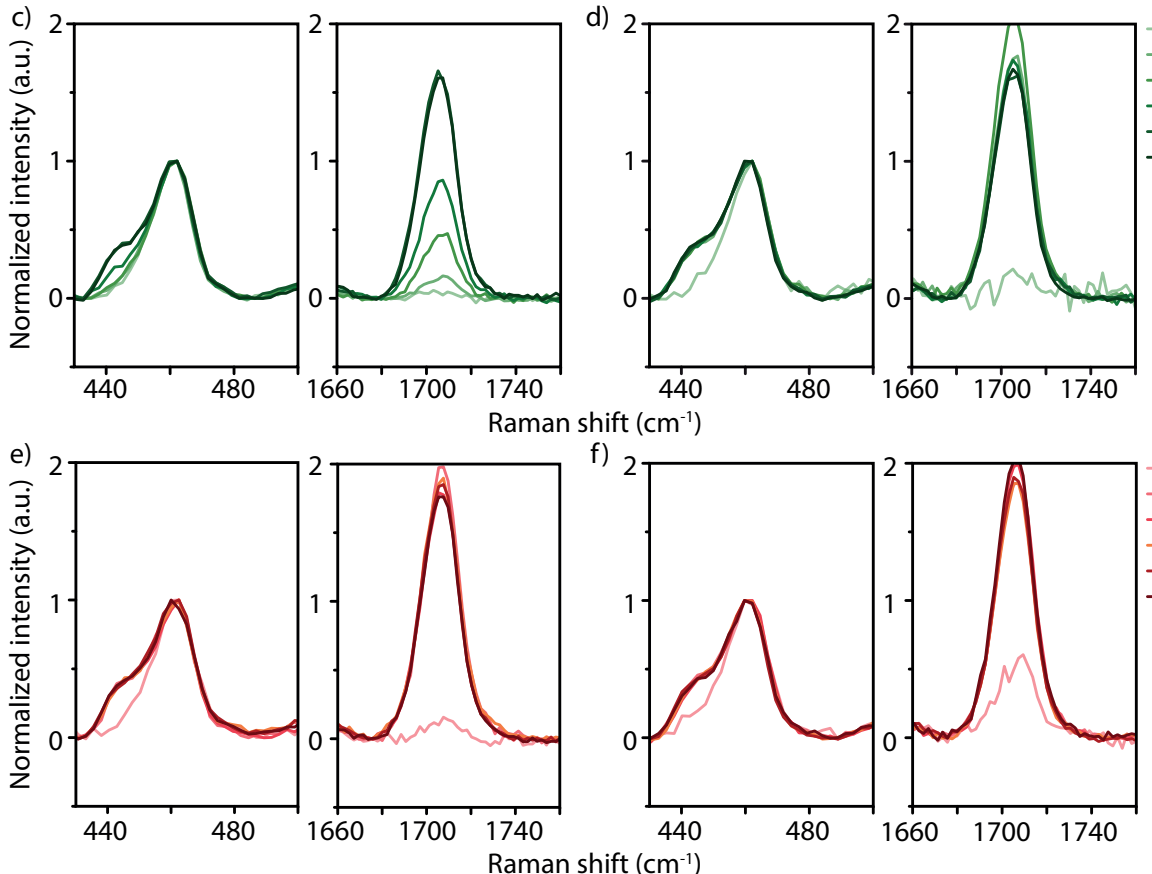
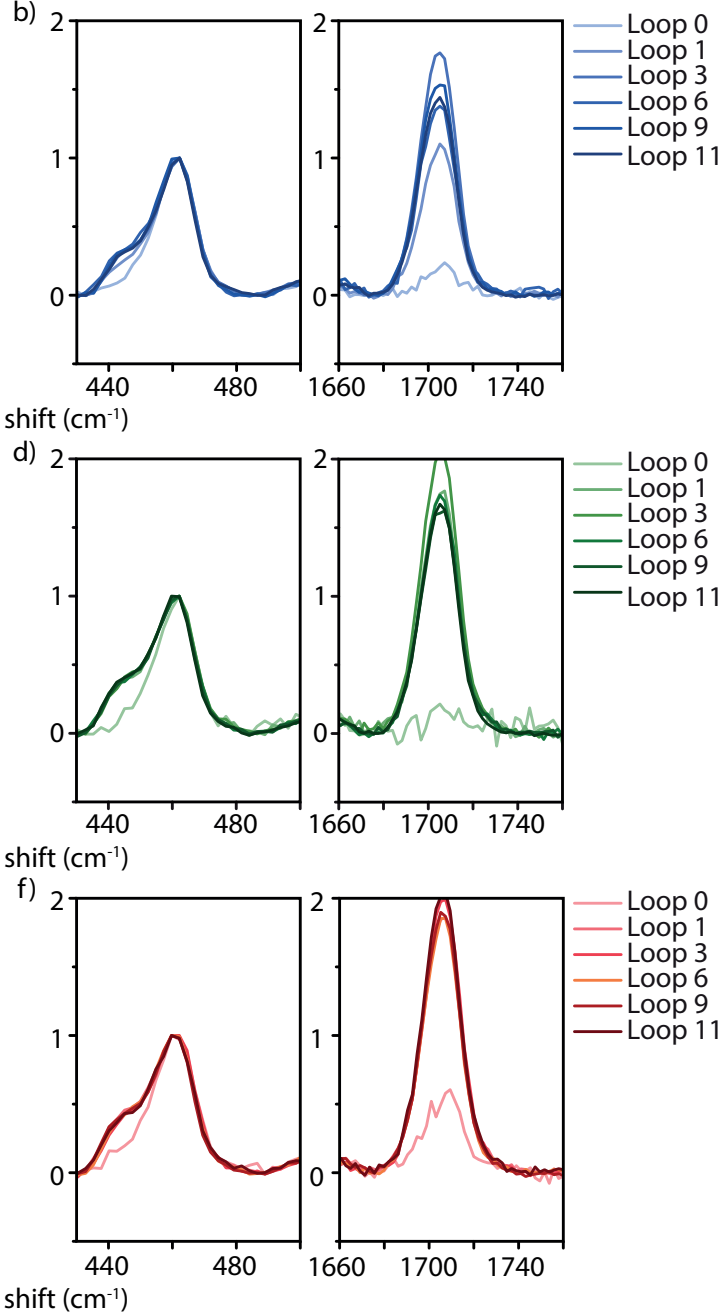

Figure 8.4: $\mathrm{CCl}_{4}$ peak $\left(460 \mathrm{~cm}^{-1}\right)$ and benzaldehyde peak $\left(1700 \mathrm{~cm}^{-1}\right)$ in the Raman spectra at different locations of the tubing at room temperature $(\mathrm{a}, \mathrm{b}), 60^{\circ} \mathrm{C}(\mathrm{c}, \mathrm{d})$ and $90{ }^{\circ} \mathrm{C}(\mathrm{e}, \mathrm{f})$ without silica $(\mathrm{a}, \mathrm{c}, \mathrm{e})$ and with $2 \mathrm{wt} \%$ silica (b, $d, f)$ in the continuous phase monitoring the deacetalization reaction. 
an effect which is most pronounced at room temperature. With the addition of silica, the benzaldehyde yield increases from $10 \%$ after 22 min without silica, to $90 \%$ with silica present. At $60^{\circ} \mathrm{C}$ full conversion was reached within $2 \mathrm{~min}$, whereas in the BS only $10 \%$ yield was observed at that time. At $90{ }^{\circ} \mathrm{C}$, the reaction was too fast to measure any differences between the BS and PE. This increase in reactivity of the PE can be addressed to the higher stability of the droplets and therefore the higher interfacial area between the droplets and the continuous phase.

a) - RT No silica • RT Silica

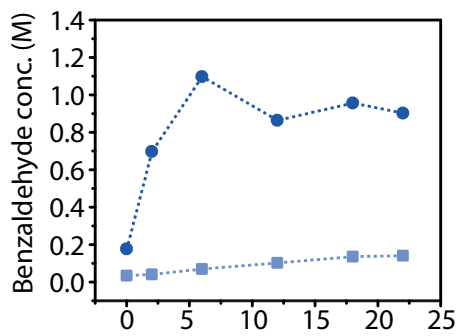

b) $=60^{\circ} \mathrm{C}$ No silica $\bullet 60^{\circ} \mathrm{C}$ Silica

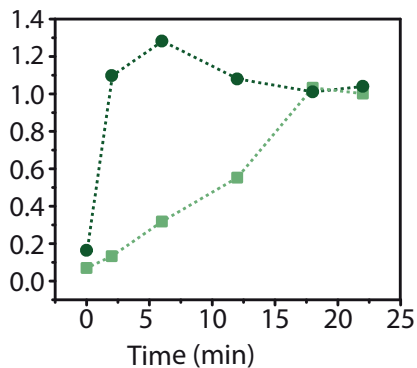

C) $=90^{\circ} \mathrm{C}$ No silica $\bullet 90^{\circ} \mathrm{C}$ Silica

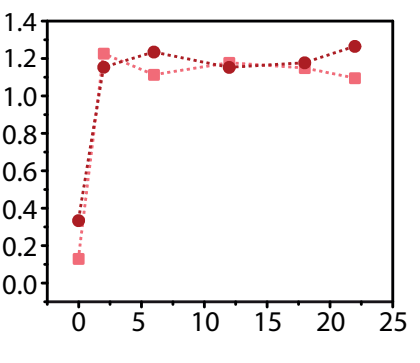

Figure 8.5: Concentration of benzaldehyde during the acid catalyzed deacetalization reaction of benzaldehyde dimethyl acetal followed over time by in-situ Raman spectroscopy at RT (a), $60{ }^{\circ} \mathrm{C}$ (b) and 90 ${ }^{\circ} \mathrm{C}$ (c). Reaction conditions: $1.0 \mathrm{M}$ benzaldehyde dimethyl acetal and $0.5 \mathrm{M} \mathrm{CCl}_{4}$ in 4-propylguaiacol (and 2 wt\% silica), $0.1 \mathrm{M} \mathrm{HCl}$ in water, total flow $25 \mu \mathrm{L} / \mathrm{min}$.

These results show the applicability of Raman spectroscopy in combination with droplet-based microfluidics. Parameters that have to be picked carefully, are among others, the choice of microreactor material, solvent and reactants. For example, the microreactor material and continuous phase, in this case FEP tubing and aqueous acid respectively, have to be compatible with the Raman measurements, meaning that it should not lead to interfering peaks in the spectrum. Also, none of the reactants or solvents used, can give rise to autofluorescence, overwhelming the Raman peaks. For single particle diagnostics, the Raman signal should be higher to allow for measuring individual droplets. This could be achieved by adding dispersed Ag or Au colloids to the droplets that lead to enhancement of the Raman signal (SERS). ${ }^{[10]}$ When all these aspects are being considered, Raman spectroscopy is a promising technique to combine with droplet-based microfluidics, due to the possible quantification of reactants and products. This technique can in future work be extended to microfluidic single particle diagnostic approaches.

\subsection{Acknowledgements}

The work described in the outlook has been performed in collaboration with Carolien Vis (Utrecht University, UU), for which both authors (Anne-Eva Nieuwelink and Carolien Vis) have contributed equally. We acknowledge Pieter Bruijnincx (UU) for scientific discussions.

\subsection{References}

[1] Y. Chevalier, M. A. Bolzinger, Colloids Surfaces A Physicochem. Eng. Asp. 2013, 439, 23-34.

[2] R. Aveyard, B. P. Binks, J. H. Clint, Adv. Colloid Interface Sci. 2003, 100-102, 503-546.

[3] Y. Chi, S. T. Scroggins, J. M. J. Fréchet, J. Am. Chem. Soc. 2008, 130, 6322-6323. 
[4] J. Faria, M. P. Ruiz, D. E. Resasco, ACS Catal. 2015, 5, 4761-4771.

[5] J. Faria, M. P. Ruiz, D. E. Resasco, Adv. Synth. Catal. 2010, 352, 2359-2364.

[6] C. M. Vis, L. C. J. Smulders, P. C. A. Bruijnincx, ChemSusChem 2019, 12, 2176-2180.

[7] H. Yang, L. Fu, L. Wei, J. Liang, B. P. Binks, J. Am. Chem. Soc. 2015, 137, 1362-1371.

[8] X. Casadevall, Chem. Commun. 2011, 47, 1936-1942.

[9] C. Albert, M. Beladjine, N. Tsapis, E. Fattal, F. Agnely, N. Huang, J. Control. Release 2019, 309, $302-332$.

[10] T.-A. Meier, R. J. Beulig, E. Klinge, M. Fuss, S. Ohla, D. Belder, Chem. Commun. 2015, 51, 8588-8591. 



\section{Chapter 9 Nederlandse Samenvatting, Publications, Presentations, Dankwoord \& Curriculum Vitae}




\subsection{Nederlandse Samenvatting}

In dit proefschrift is onderzoek beschreven naar het gebruik van microfluïdica en spectroscopie voor de diagnostiek van individuele katalysatordeeltjes. Een zin met heel veel informatie, die waarschijnlijk ook veel vragen oproept, vooral bij de niet-specialist. In dit deelhoofdstuk zal ik eerst de woorden katalysator, microfluïdica en spectroscopie verder uitleggen en vertellen wat het verband is tussen deze woorden binnen de context van dit proefschrift.

Katalysatoren zijn belangrijke materialen in de chemische industrie om een chemische reactie op een effectieve manier plaats te laten vinden zonder daarbij zelf verbruikt te worden. Je kunt ze hierdoor steeds opnieuw gebruiken in een zogeheten katalytische cyclus. Daarnaast is een katalysator erg goed in het sturen van een chemische reactie in de richting van gewenste producten. Een katalysator is hier zo goed in, omdat het de activeringsenergie van een chemische reactie verlaagt. ${ }^{[1]}$ De activeringsenergie is de hoeveelheid energie die nodig is om een chemische reactie te laten verlopen. Een analogie die hier vaak voor gebruikt wordt is een hoge berg met zowel een moeilijk begaanbaar bergweggetje als een tunnel. De tunnel maakt het makkelijker om van de ene kant van de berg (de chemische reactanten) naar de andere kant van de berg (de reactieproducten) te komen. In deze analogie is het nemen van de bergweg de hoge activeringsenergie zonder katalysator en het nemen van de tunneldelagereactiveringsenergie met katalysator.

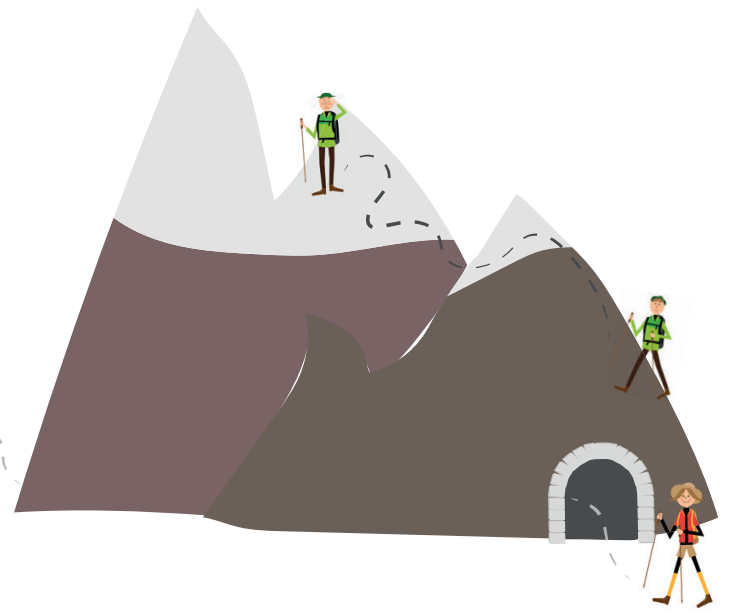

Figuur 9.1: De tunnel wordt gebruikt, maar niet verbruikt. Je komt op de goede plek uit en verbruikt daarbij minder energie.

Meer dan $80 \%$ van alle chemische producten zien tijdens hun productieprocessen ten minste één katalysator. ${ }^{[2,3]}$ Vier belangrijke en onderling afhankelijke aspecten van (heterogene) katalysatoren zijn activiteit, stabiliteit, selectiviteit en toegankelijkheid. Niet alles van een katalysatordeeltje doet mee aan de chemische reactie, maar alleen de actieve centra. Dit kan bijvoorbeeld een plek op een metalen nanodeeltje of een zuur/ basische plaats zijn. De rest van het (vaak poreuze) deeltje dient onder andere voor het vergroten van het beschikbare oppervlak en mechanische stabiliteit. De mate van activiteit van een katalysator wordt bepaald door zijn omzetfrequentie, maar een zeer actieve katalysator is nutteloos wanneer een niet-gewenst product wordt geproduceerd. De mate waarin een katalysator het juiste of gewenste product vormt, wordt de selectiviteit genoemd. Omgekeerd is een zeer selectieve katalysator met een lage activiteit even nutteloos. De toegankelijkheid van de actieve centra is van het grootste belang voor 
een hoge katalytische activiteit en is te verbeteren door katalysatordeeltjes poreuzer te maken. Het verhogen van de porositeit kan echter ten koste gaan van de stabiliteit van de deeltjes. ${ }^{[1,2]} \mathrm{Om}$ tot een optimaal katalytisch proces te komen, is uitgebreid onderzoek nodig om het samenspel van deze vier parameters te optimaliseren. Met de toenemende kennis die we hebben over de structuur, samenstelling en werking van vaste katalysatoren, weten we dat deze complexe materialen onderling zeer verschillend kunnen zijn. ${ }^{[4,5]}$ Deze heterogeniteit binnen de katalysatordeeltjes belemmert vaak de analyse van katalysatoren met zogeheten bulk-methoden, omdat bij het gebruik van een grote hoeveelheid deeltjes alleen gemiddelde waardes worden gevonden voor bijvoorbeeld activiteit of porositeit. De analyse van individuele katalysatordeeltjes is wél heel gedetailleerd, maar brengt een gebrek aan statistische relevantie met zich mee als er niet genoeg deeltjes worden geanalyseerd. Het is kostbaar om een statistisch relevante hoeveelheid deeltjes individueel te analyseren. ${ }^{[6]}$ We moeten daarom de kloof tussen de analyse van afzonderlijke katalysatordeeltjes en bulkmethodes overbruggen door de juiste technieken te ontwikkelen die individuele katalysatordeeltjes kunnen screenen op een hoge snelheid. Dit laatste noemen we ook wel high-throughput analysetechnieken.

In dit proefschrift worden verschillende methoden gebruikt om individuele katalystordeeltjes te testen met gebruik van kleine reactoren (druppelmicrofluïdica) en spectroscopie. Deze reactoren werken met volumes in de ordegrootte van nanoliters. Vloeistoffen en gassen gedragen zich op de micrometerschaal (en kleiner) natuurkundig gezien anders dan we gewend zijn in het dagelijks leven. Het bestuderen van dit gedrag
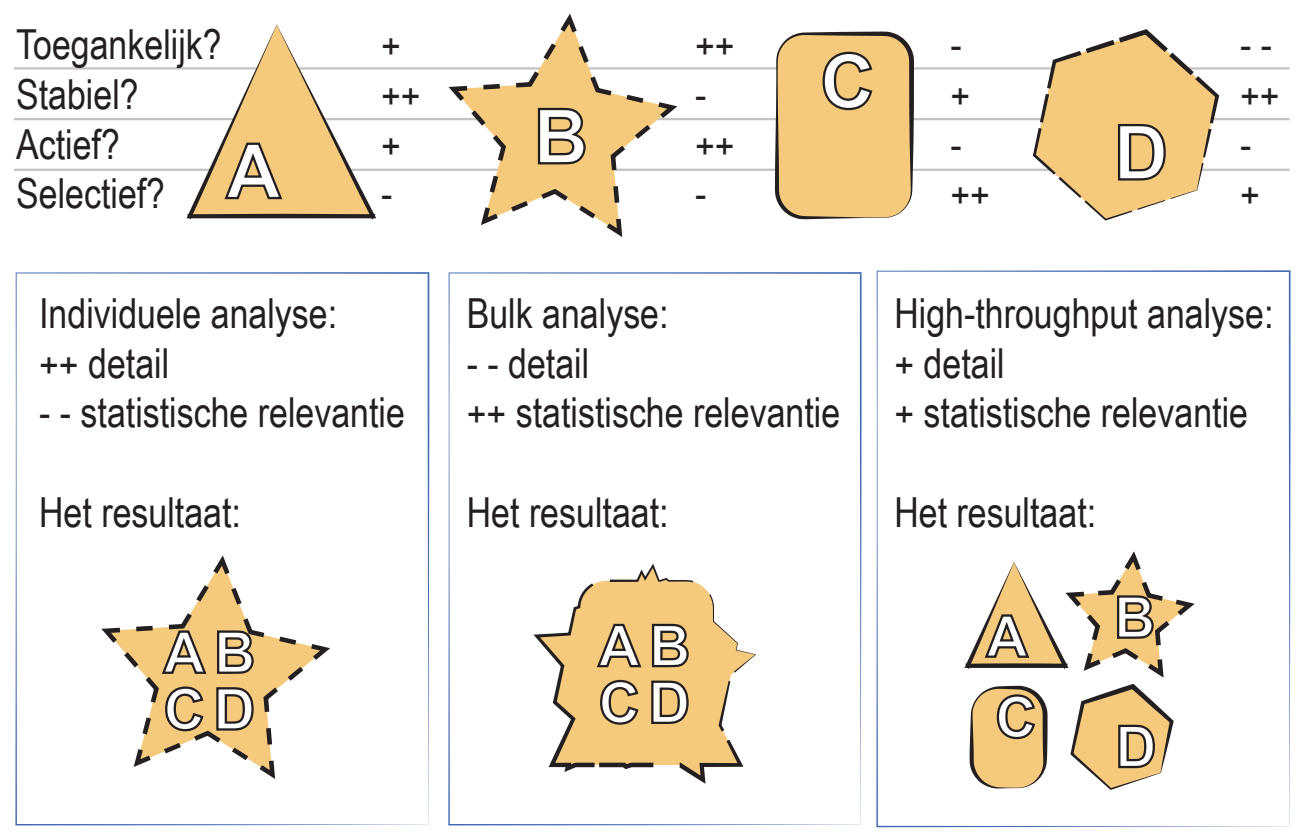

Figuur 9.2: Elke vorm in bovenstaand figuur staat voor een individueel katalysatordeeltje. Bij een individuele analyse doe je alsof je analyse representatief voor het hele monster is. Bij een bulk analyse vallen juist alle details weg. Bij een high-throughput analyse analyseer je veel deeltjes op een gemiddeld detailniveau. Daardoor zie je bij deze techniek het best de verschillen tussen alle deeltjes in je monster. 
of inzetten van deze kleine lengteschaal voor chemische of biologische doeleinden heet microfluïdica. Voorbeelden hiervan zijn de printtechnologie en het testen van (menselijke) cellen, DNA of bloed. ${ }^{[7,8]}$ Katalysatordeeltjes delen een aantal eigenschappen met biologische cellen, ze zijn bijvoorbeeld ongeveer even groot (50 $\mu \mathrm{m}$ en $10 \mu \mathrm{m}$ respectievelijk) en ze zijn qua chemische activiteit onderling vaak erg verschillend van elkaar. We hebben dan ook geprobeerd om technieken die al gebruikt worden voor het sorteren van individuele cellen in te zetten voor het sorteren van individuele katalysatordeeltjes. Dit heeft als voordeel dat we veel deeltjes in korte tijd kunnen analyseren (high-throughput) waardoor we veel informatie krijgen over individuele deeltjes. Dit verbetert de statistische relevantie van de analyse. We gebruiken hiervoor spectroscopie; omschreven als "het waarnemen van een spectrum met behulp van een

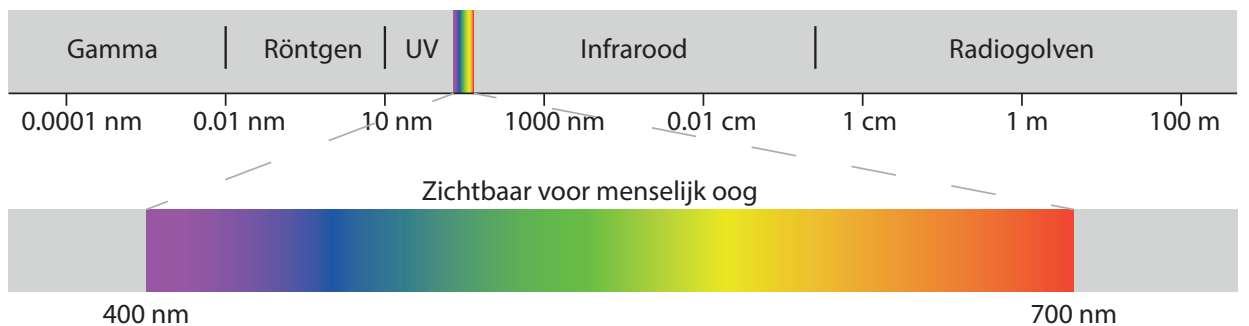

UV-vis absorptiespectroscopie, je straalt wit licht op je monster

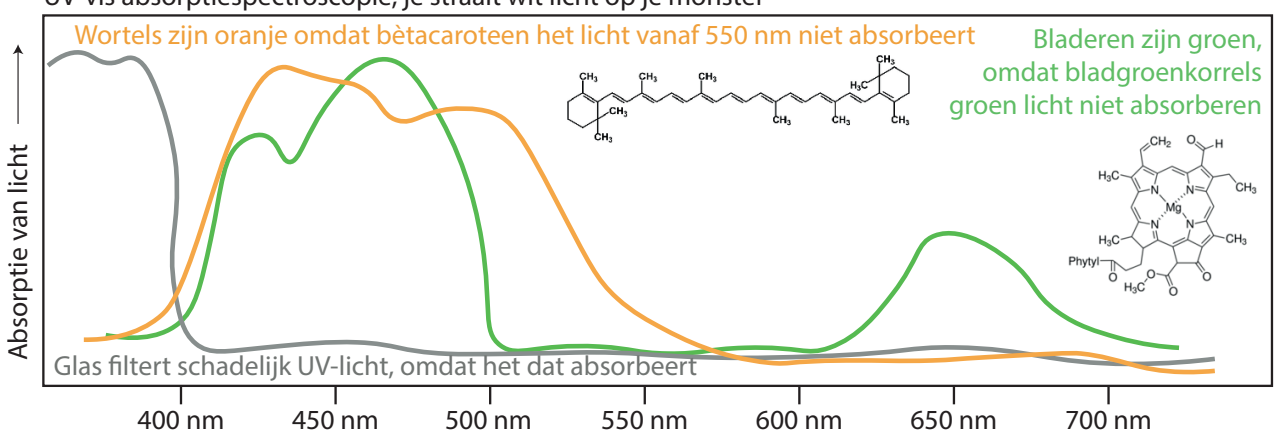

Fluorescentiespectroscopie, je straalt licht met een specifieke golflengte op je monster

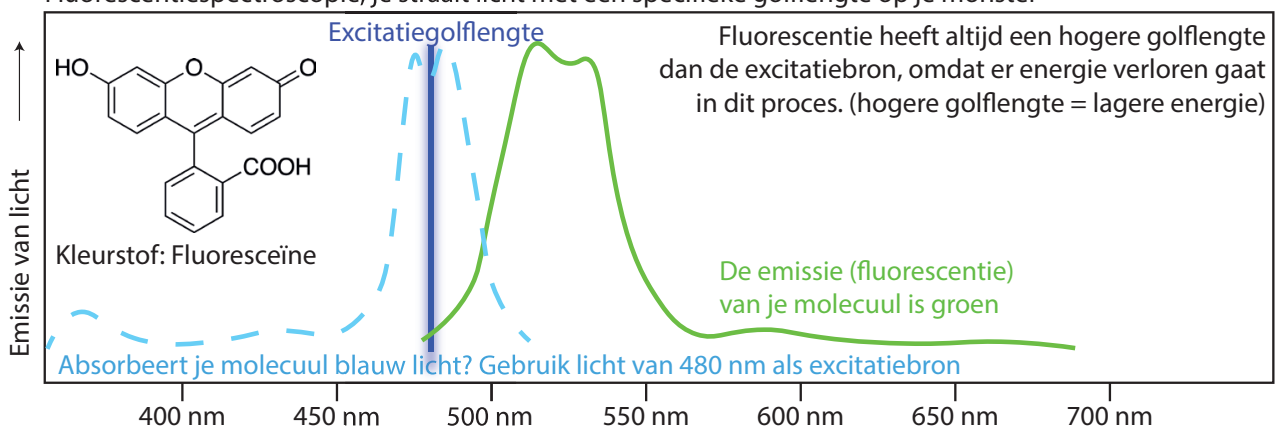

Figuur 9.3: UV-vis absorptie en fluorescentie in een notendop, uitgelegd aan de hand van enkele voorbeelden. Bij het analyseren van katalysatordeeltjes kan een kleurstof zoals bijvoorbeeld fluoresceïne gebruikt worden, die dan afhankelijk van hoeveelheid kleurstof die tijdens de katalytische reactie gevormd wordt of, een hoog of laag fluorescent signaal geeft. 
spectroscoop”. Het woord 'spectroscopie' is afgeleid van het Latijnse 'spectrum' (beeld) en het Griekse 'skopeoo' (bekijken) en wordt gebruikt om technieken aan te duiden die met behulp van de absorptie of emissie van licht naar de chemische eigenschappen van een deeltje kunnen kijken. Op basis van deze eigenschappen zie je meer of minder kleur, fluorescentie of bijvoorbeeld UV-absorptie. Deze waarde kan vervolgens gebruikt worden om het deeltje op te sorteren.

Een katalysator die erg geschikt is als showcase voor het sorteren van afzonderlijke deeltjes met behulp van microfluïdische reactoren, is de Fluid Catalytic Cracking (FCC)katalysator ${ }^{[9,10]}$ FCC is een van de processen in een olieraffinaderij die het gedistilleerde residu van ruwe olie gebruikt als grondstof om daar bijvoorbeeld benzine van te maken. Het FCC-proces werkt sinds de jaren 40 in zijn huidige vorm. ${ }^{[1]}$ De gebruikte katalysator bestaat uit poreuze deeltjes met een gemiddelde diameter van 50-150 $\mu \mathrm{m}$ en bevat onder andere klei, siliciumoxide en aluminiumoxide. Deze deeltjes hebben ongeveer de maat van een zandkorrel en zijn gemaakt van ongeveer dezelfde chemische elementen, maar verder dan dat zijn er weinig overeenkomsten te vinden. FCC-deeltjes zijn namelijk poreus en bevatten het synthetische mineraal 'zeoliet' dat zorgt voor de katalytische activiteit. Deze actieve component in de katalysatordeeltjes, het zeoliet, werkt als Brønsted-zuur. De term Brønsted-zuur komt van de gelijknamige ontdekker, die erachter kwam dat sommige moleculaire structuren een vrij proton hebben $\left(\mathrm{H}^{+}\right)$wat zorgt voor een lage pH (net als azijnzuur, salpeterzuur, citroenzuur, enz.). De meeste Brønstedzuren zijn vloeistoffen, maar een zeoliet is een zogenaamd vast zuur. Het mineraal met vrije protonen is de katalysator die zorgt voor het kraken (in stukjes knippen) van de oliemoleculen. Een leuk weetje: de katalysatordeeltjes zijn zo gemaakt (qua maat en

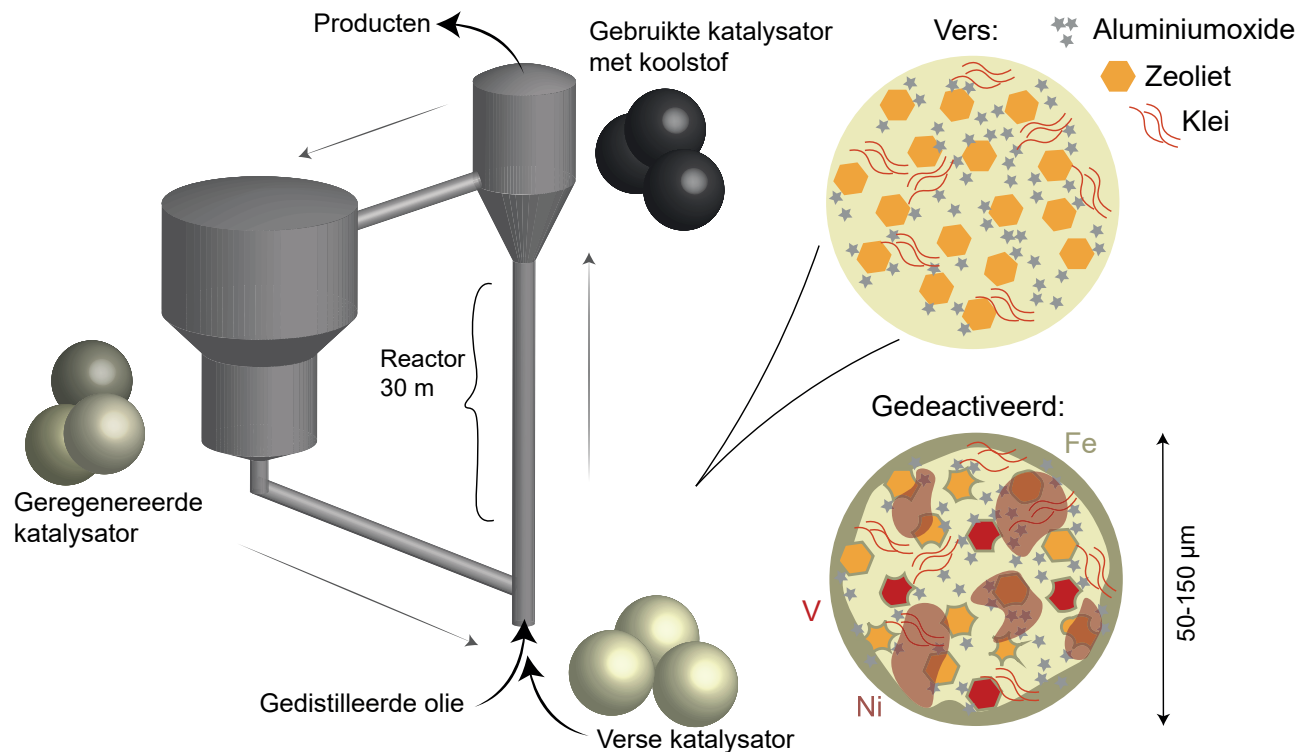

Figuur 9.4: In de Fluid Catalytic Cracking reactor gaat de katalysator rond, waarbij er telkens een deel van de geregenereerde katalysator wordt vervangen met verse katalysator. Hierdoor ontstaat een mengsel van katalysatordeeltjes met een verschillende deactivatiegraad (equilibrium catalyst, ECAT). 
vorm) dat het mengsel van hete katalysator en gedistilleerde aardolie zich als een vloeistof gedraagt; vandaar de term Fluid Catalytic Cracking (FCC). ${ }^{[12,13]}$ In de FCC-reactor wordt het kraken uitgevoerd door de voorverhitte FCC-katalysator samen met de ruwe olie in hoge kolom te pompen, waarna de gevormde producten worden gescheiden van de katalysator. Tijdens de katalytische reactie wordt een koolstoflaagje (roet) afgezet op de katalysatordeeltjes, waardoor de actieve plaatsen geblokkeerd worden. De gebruikte katalysator wordt daarom onderworpen aan een regeneratieprocedure door de deeltjes te verhitten in een zuurstofrijke omgeving, om zo het koolstoflaagje weg te branden.

Hierna zijn de FCC-deeltjes klaar om een nieuwe katalytische cyclus te ondergaan waarbij ze aardolie kraken. ${ }^{[14]}$ De FCC-deeltjes kunnen echter ook onomkeerbaar worden gedeactiveerd vanwege de ophoping van metalen afkomstig van deze aardolie, waarbij nikkel, ijzer en vanadium de meest beruchte en vaakst voorkomende zijn. Ook het verwijderen van het koolstoflaagje tijdens de regeneratie, kan als gevolg hebben dat de katalysatordeeltjes langzaam kapotgaan. Om de totale activiteit in een industriële FCCreactor te behouden, wordt een deel van het gebruikte katalysatormateriaal constant vervangen door verse FCC-katalysatordeeltjes, wat leidt tot een evenwicht in de reactor met een mengsel van FCC-katalysatordeeltjes met verschillende leeftijden en mate van deactivatie. Dit mengsel wordt de 'equilibrium catalyst' genoemd, verder aangeduid als ECAT. De katalysatordeeltjes in een ECAT verschillen erg van elkaar. Dit bemoeilijkt de studie van deze industrieel gebruikte katalysatordeeltjes. Daarom is er behoefte aan een nieuwe methode om ECAT-deeltjes te sorteren op hun katalytische activiteit of hun hoeveelheid metalen die zorgt voor deactivatie. In eerdere onderzoeken om enkele FCCdeeltjes te testen op hun activiteit werd een molecuul gebruikt dat een fluorescent product vormt als het reageert op een Brønsted zuur in een zeoliet. De fluorescentie is daardoor een maatstaaf voor de activiteit van het deeltje. Verder kan de samenstelling van een FCCdeeltje (en daarmee dus ook de hoeveelheid deactiverende metalen) gemeten worden met een techniek die Röntgenfluorescentie (XRF) spectrometrie heet. Het te meten deeltje wordt bij deze techniek bestraald met Röntgenstraling. Dit veroorzaakt Röntgenstraling met een langere golflengte, omdat de atomen in het FCC-deeltje zorgen voor een verlies aan energie. De golflengte van het uitgezonden licht is karakteristiek voor het atoom dat verantwoordelijk is voor het energieverlies. Omdat elk atoom een unieke samenstelling van protonen, neutronen en elektronen heeft en daarmee dus een uniek XRF-spectrum, kun je zo in principe elk metaal in je FCC-deeltje analyseren.

In hoofdstuk 2 is een nieuw ontwikkelde 3D-geprinte microfluïdische chip gebruikt om ECAT-deeltjes te sorteren in 5 fracties, op basis van hun magnetisch moment. Dit is mogelijk door een magneet op precieze afstand van een microfluïdisch kanaal te brengen en zo magnetische ECAT-deeltjes van hun rechte pad af te buigen. De trends in magnetisch moment van de gesorteerde FCC-deeltjes correleren met hun ijzergehalte. Wij verwachtten dat een hoger ijzergehalte leidt tot een lagere katalytische activiteit, omdat ijzer een van de metalen is die een FCC-deeltje zal deactiveren. Echter, de spreiding in activiteit van elke fractie is nog steeds groot en niet alle sterk magnetische deeltjes vertonen een hoge mate 
van deactivatie. Dit zou kunnen komen doordat ijzer zich ophoopt zonder het deeltje te deactiveren, in de vorm van grote ijzerclusters die wél zorgen voor een hoog magnetisch moment, maar niet voor het blokkeren van de poreuze structuur van een FCC-deeltje.

Hoofdstuk 3 laat zien dat het correleren van informatie uit meerdere analysetechnieken per deeltje waardevolle nieuwe inzichten oplevert in de heterogeniteit van een ECAT, aangezien een ECAT bestaat uit een mengsel van katalysatordeeltjes met een verschillende graad van deactivatie. Bovendien voegt de mogelijkheid om meerdere deeltjes in één keer te meten, statistische relevantie toe aan de verkregen gegevens. Waar bulkmetingen inzicht kunnen geven

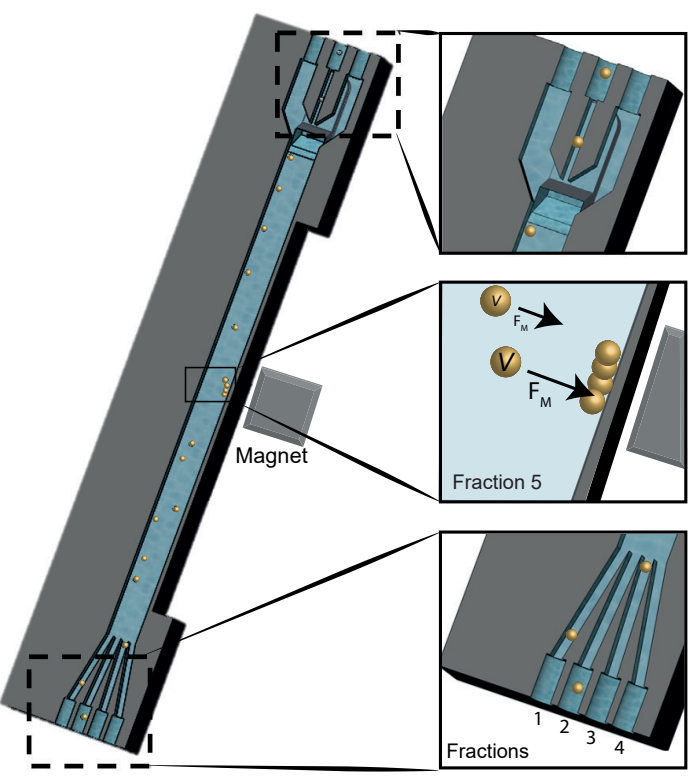

Figuur 9.6: Hoe sterker het magnetisch moment van een ECAT-deeltje, hoe verder het richting de magneet getrokken wordt. Van fractie 1 tot 5 loopt het magnetisch moment en daarmee het gehalte ijzer op.

in de trends tussen zes, op dichtheid gescheiden ECAT-fracties die worden onderzocht, kan de diagnostiek van afzonderlijke deeltjes zelfs binnen elke fractie trends weergeven. De grote heterogeniteit tussen de ECAT-deeltjes werd zichtbaar gemaakt door Röntgenfluorescentie metingen te doen die de hoeveelheid ijzer en nikkel weergeeft. Deze

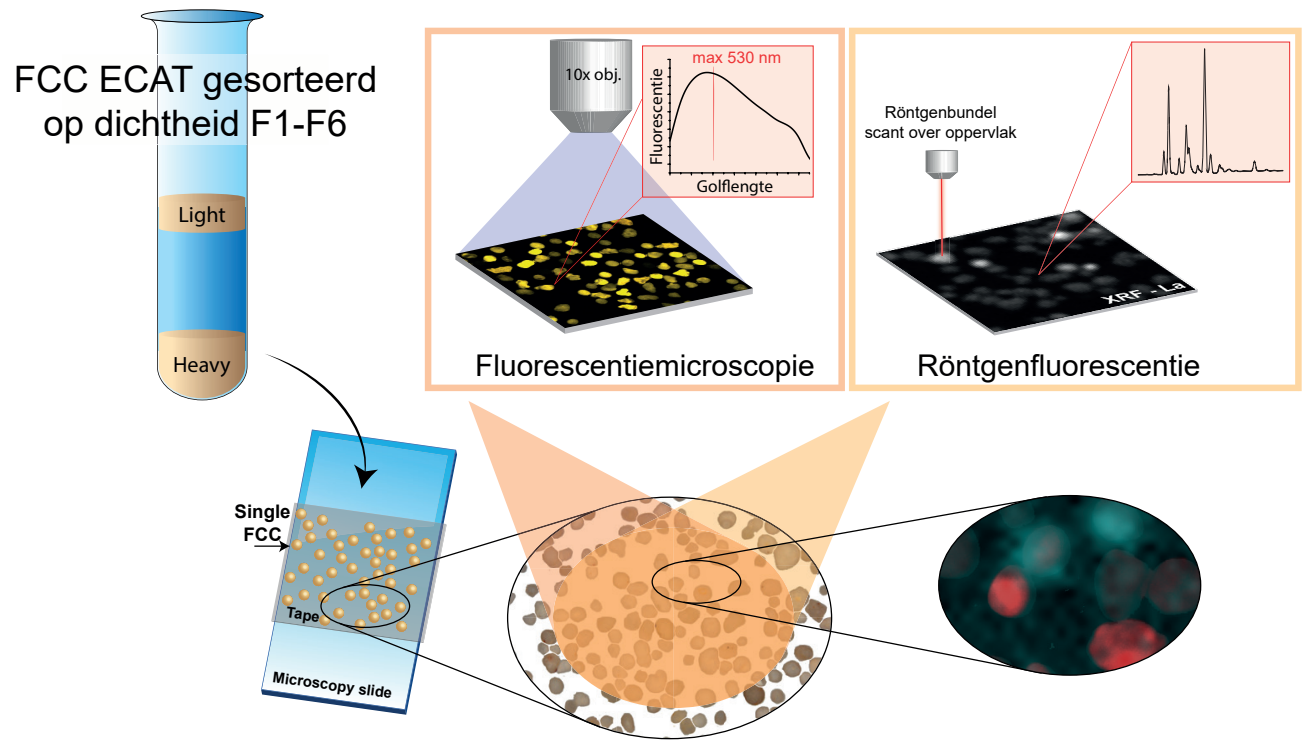

Figuur 9.5: 9.6: ECAT-deeltjes werden vastgemaakt op dubbelzijdig tape, om ze te kunnen analyseren met twee technieken, waarvan de resultaten over elkaar heen werden gelegd. 
data werd gecorreleerd aan de activiteit en porositeit per deeltje. De mate van deactivatie werd zo per deeltje gekoppeld aan het nikkelgehalte, in die zin dat deeltjes met een hoog nikkelgehalte geen hoge katalytische activiteit vertonen.

In hoofdstuk 4 en 5 zijn twee druppel-gebaseerde microreactoren beschreven die kunnen worden gebruikt voor chemische analyse bij verhoogde temperaturen en druk. In hoofdstuk 4 wordt een druppelmicroreactor beschreven die in staat is om monodisperse druppels, druppels met allemaal dezelfde maat, te vormen en te verwarmen tot 120 ${ }^{\circ} \mathrm{C}$ bij drukken tot 7 bar, waarbij de druppels stabiel blijven. Deze microreactor werd gebruikt voor high-throughput screening van individuele ECAT-deeltjes, op basis van hun katalytische activiteit. De screening werd in-situ uitgevoerd bij temperaturen tot 95 ${ }^{\circ} \mathrm{C}$ met behulp van het fluorescente oligomeer (meerdere monomeren aan elkaar) van 4-methoxystyreen als indicatie voor de reactiviteit van de katalysator. 'In-situ' wil zeggen dat de oligomerisatiereactie van 4-methoxystyreen in de microreactor werd uitgevoerd en dat de fluorescentie ervan in dezelfde microreactor werd gemeten. In totaal werden
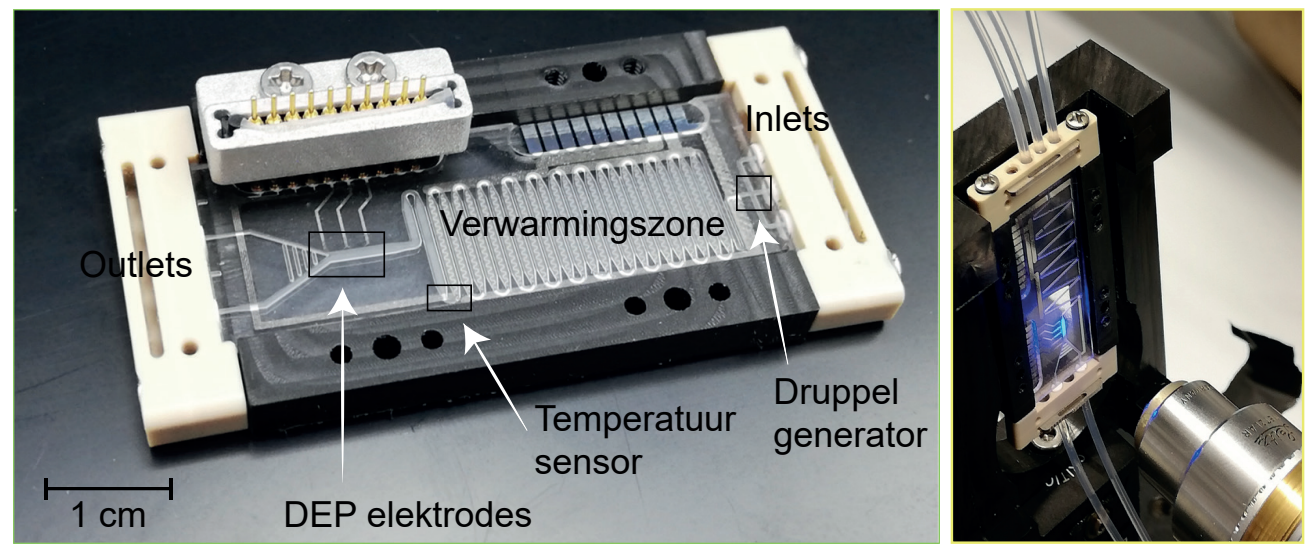

Figuur 9.7: De microreactor die gebruikt is in hoofdstuk 5 is gemaakt van glas en zit ingeklemd in een houder om de toevoerbuisjes aan te sluiten. In de opstelling hangt de microreactor verticaal (zie foto rechts).

1000 deeltjes gedetecteerd met een gemiddelde snelheid van 1 deeltje per 2,4 seconden.

De microreactor beschreven in hoofdstuk 5 heeft daarnaast ook een geïmplementeerd sorteersysteem dat gebruik maakt van diëlektroforese (DEP). FCC-deeltjes gekleurd met het fluorescente oligomeer van 4-fluorostyreen werden gesorteerd op basis van hun fluorescentie-intensiteit en dus hun Brønsted-zuurtegraad. Dit sorteren met behulp van DEP houdt in dat een druppel met daarin een fluorescent ECAT-deeltje waarvan het fluorescente signaal hoog genoeg is, in een ander microkanaal wordt geduwd doormiddel van een elektrisch veld. Dit veld wordt $90 \mathrm{~ms}$ ingeschakeld om alleen de juiste druppel uit zijn baan te duwen. Na het sorteren en verzamelen van de FCC-deeltjes werden ze geanalyseerd met behulp van fluorescentiemicroscopie en micro-röntgenfluorescentie ( $\mu \mathrm{XRF}$ ) op dezelfde manier als beschreven in hoofdstuk 3. Deze analyse na het sorteren liet zien dat de niet-geselecteerde (en dus minder fluorescente) deeltjes hogere nikkelgehaltes bevatten dan de geselecteerde deeltjes. Aangezien nikkel niet aanwezig is in verse deeltjes en alleen kan worden verzameld door tijd door te brengen in een 

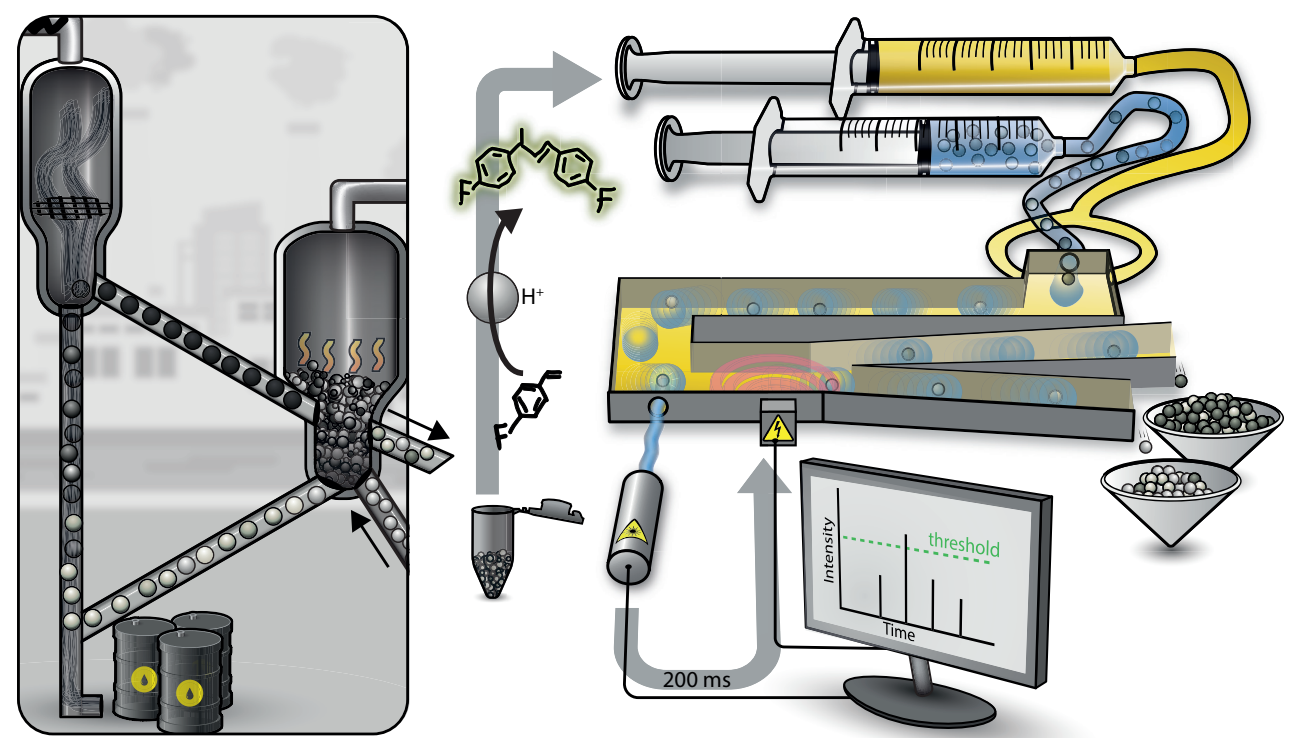

Figuur 9.8: De ECAT deeltjes worden gekleurd met 4-fluorostyreen en gesorteerd op basis van hun fluorescentie. Als de waarde boven een bepaalde grenswaarde komt, wordt de druppel met het betreffende deeltje binnen 200 milliseconde een ander kanaal in geduwd met behulp van diëlektroforese.

industriële reactor, geeft dit aan dat de niet-geselecteerde deeltjes de meer verouderde deeltjes zijn.

De technieken die waren gericht op de analyse van FCC-deeltjes, zoals beschreven in hoofdstukken 2-5, tonen verschillende benaderingen om de mate van deactivatie van ECAT-deeltjes te analyseren aan de hand van de ophoping van metalen (d.w.z. ijzer en nikkel), hun zuurtegraad en de bereikbaarheid van deze zure plaatsen. Ook werden de deeltjes op basis van deze eigenschappen gesorteerd. Het combineren van deze sorteeren analysetechnieken, levert een diagnostiekplatform dat de gewenste katalysatordeeltjes uit een monster kan selecteren. Dit maakt het mogelijk om de meest actieve deeltjes te selecteren, om te bepalen waarom deze zo goed werken. Dit zou kunnen leiden tot nieuwe ontwerpregels om actievere en/of stabielere FCC-katalysatoren voor verschillende ruwe aardoliebronnen te maken.

Hoofdstuk 6 en 7 zijn gewijd aan andere katalytische systemen dan het kraken van ruwe olie. In hoofdstuk 6 tonen we een proof-of-concept van een microreactor gemaakt van het polymeer polydimethylsiloxaan (PDMS) voor katalytische reacties waarvan een van de reactanten een gas is, in dit geval waterstof $\left(\mathrm{H}_{2}\right)$. De hydrogenatie (in vloeibare fase) van methyleen blauw (MB) wordt aangetoond doormiddel van de kleurverandering die $\mathrm{MB}$ ondergaat bij de hydrogenatie naar leuco-methyleen blauw. In een vloeistofkanaal worden druppels gevormd die 20 ppm MB in ethanol bevatten. In deze druppels zit een katalysatordeeltje dat bestaat uit palladium nanodeeltjes die afgezet zijn op een silicabol van $40 \mu \mathrm{m}$ in doorsnee. Het vloeistofkanaal wordt geflankeerd door twee gaskanalen met $\mathrm{H}_{2}$, gescheiden door $50 \mu \mathrm{m}$ PDMS-wanden. Vanwege de hoge permeabiliteit van PDMS diffundeert $\mathrm{H} 2$ naar de vloeistofkanalen en kan de door palladium gekatalyseerde 
a)

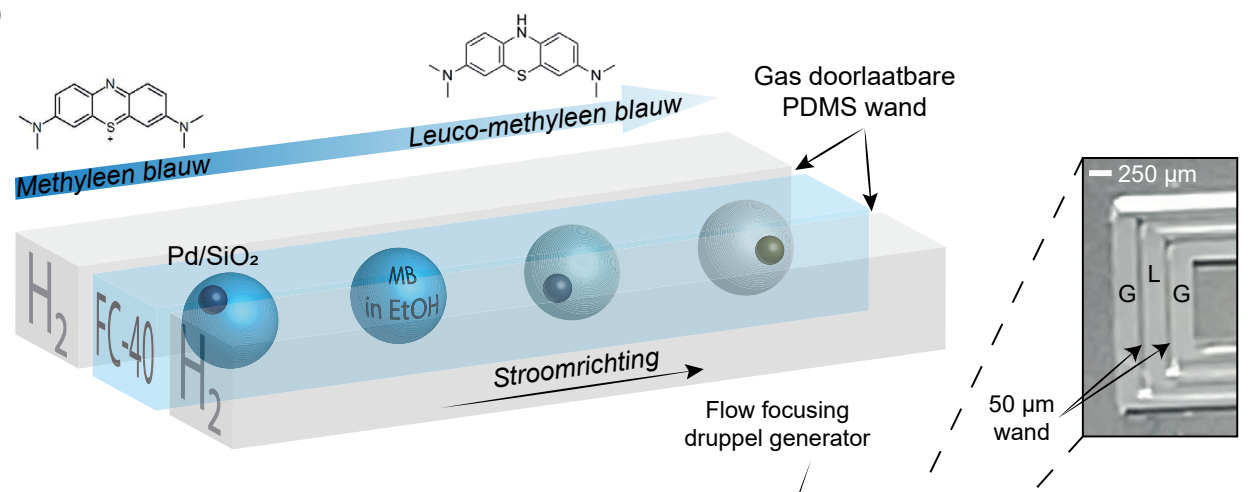

b)

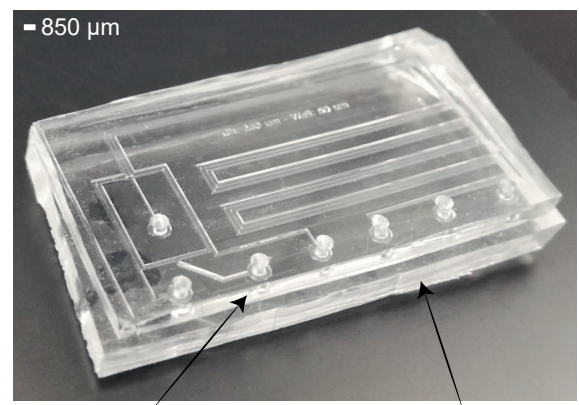

PDMS top substraat

PDMS bodem substraat

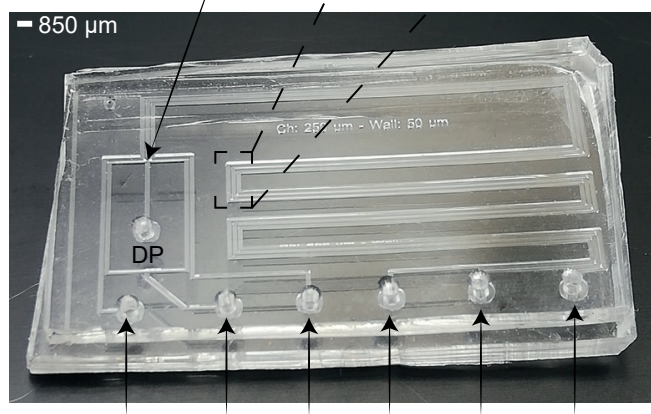

met kanalen

Figuur 9.9: De microreactor in a) laat drie kanalen naast elkaar zien. De buitenste twee worden gebruikt voor waterstof, dat door de dunne gas doorlaatbare PDMS wand in het middenkanaal met de druppels terecht komt. De microreactor is volledig doorzichtig zoals te zien is in b) waarbij DP=droplet phase, GI= gas inlet, $\mathrm{CP}=$ continuous phase, $\mathrm{GO}=$ gas outlet en $\mathrm{O}=$ vloeistof outlet..

hydrogenatie van MB bij kamertemperatuur plaatsvinden.

Verdere ontwikkeling van deze systemen zou hun bruikbaarheid verder kunnen vergroten. Door bijvoorbeeld de PDMS-chip uit hoofdstuk 6 op een verwarmingsplaatje te plaatsen, kunnen reacties bij verhoogde temperaturen worden uitgevoerd. De thermische stabiliteit van PDMS maakt het mogelijk tot $400{ }^{\circ} \mathrm{C}$ te verwarmen voordat het begint af te breken (echter onstaan er bij lagere temperatuur al problemen zoals het koken van oplosmiddelen of een niet-uniforme verwarming van de microreactor). Bovendien laten de optische eigenschappen van PDMS toe om chemische reacties met (laser) licht te monitoren, waardoor in-line UV-vis of Raman spectroscopie kan worden uitgevoerd. Deze spectroscopische technieken moeten zodanig worden geoptimaliseerd dat ze snel kunnen werken en afzonderlijke druppels met hoge stroomsnelheden kunnen detecteren. Meer geavanceerde microreactorontwerpen met microfluïdische druppelsorteerders kunnen vervolgens worden geïmplementeerd om de interessante katalysatordeeltjes te sorteren.

Hoofdstuk 7 beschrijft een goedkope en eenvoudige buis-in-buis microreactor, gemaakt van een kunststofslang (FEP), voor de diagnostiek van afzonderlijke katalysatordeeltjes. Vanwege de goedkope en eenvoudige fabricage van deze microreactor, 
kan het microkanaal worden aangepast aan de verschillende reactanten of reactietijden die nodig zijn voor de katalytische reactie, door het aantal gebruikte T-stukken te vergroten of de FEP-slang te verlengen. Het systeem werd getest op de synthese van een fluorescente benzocoumarine die via een basisch gekatalyseerde Knoevenagelcondensatie gevormd werd. Microdruppeltjes van $0,7 \mu$ werden gevormd door een dispersie van katalysatordeeltjes te mengen met vloeibare reactanten bij een T-splitsing. In de volgende stap werden druppeltjes van de gecombineerde reactant- en katalysatorfase gecreëerd in een continue fase van siliconenolie. Vanwege de mechanische instabiliteit van de katalysatordeeltjes konden de individuele deeltjes niet geanalyseerd worden per druppel. Fluorescentie-intensiteitsmetingen werden echter uitgevoerd om de reactiviteit van vier verschillende katalysatoren te screenen voor de synthese van de fluorescerende coumarine.

[1] L. Lefferts, E. Hensen, H. Niemantsverdriet, in Catal. an Integr. Textb. Students (Eds.: U. Hanefeld, L. Lefferts), Wiley-VCH Weinheim, 2018, pp. 15-70.

[2] M. Boudart, in Handb. Heterog. Catal. (Eds.: G. Ertl, H. Knözinger, J. Weitkamp), Wiley-VCH Weinheim, 2008.

[3] J. Hagen, Industrial Catalysis, Wiley-VCH, Weinheim, 2015.

[4] B. M. Weckhuysen, Angew. Chem. Int. Ed. 2009, 48, 4910-4943.

[5] E. Plessers, I. Stassen, S. P. Sree, K. P. F. Janssen, H. Yuan, J. Martens, J. Hofkens, D. De Vos, M. B. J. Roeffaers, ACS Catal. 2015, 5, 6690-6695.

[6] I. L. C. Buurmans, B. M. Weckhuysen, Nat. Chem. 2012, 4, 873-886.

[7] A. Manz, N. Graber, H. M. Widmers, Sensors Actuators B Chem. 1990, 1, 244-248.

[8] G. M. Whitesides, Nature 2006, 442, 368-373.

[9] H. S. Cerqueira, G. Caeiro, L. Costa, F. R. Ribeiro, J. Mol. Catal. A Chem. 2008, 292, 1-13.

[10] E. T. C. Vogt, B. M. Weckhuysen, Chem. Soc. Rev. 2015, 44, 7342-7370.

[11] "A National Historic Chemical Landmark: The Houdry Process," 1996.

[12] W. Letzsch, in Handb. Pet. Process. (Ed.: S.A. Treese), Springer, Cham, 2015, pp. 262-312.

[13] R. Sadeghbeigi, Fluid Catalytic Cracking Handbook. An Expert Guide to the Practical Operation, Design and Optimization of FCC Units., Elsevier, Amsterdam, 2012.

[14] J. Biswas, I. E. Maxwell, Appl. Catal. 1990, 63, 197-258. 


\subsection{Publications \& Presentations}

\subsubsection{Publications in peer reviewed journals}

A.-E. Nieuwelink*, J.C. Vollenbroek*, A.C. Ferreira de Abreu, R.M. Tiggelaar, A. van den Berg, M. Odijk, B.M. Weckhuysen, Single Catalyst Particle Diagnostics in a Microreactor for Performing Multiphase Hydrogenation Reactions, (2020) Faraday Discuss., https://doi. org/10.1039/D0FD00006J.

A.-E. Nieuwelink*, M.E.Z. Velthoen*, Y. Nederstigt, K. Jagtenberg, F. Meirer, B.M. Weckhuysen, Single Particle Essays to Determine Heterogeneities within Fluid Catalytic Cracking Catalysts, (2020) Chem. Eur. J, 26, 8546-8554.

M. Solsona*, A.-E. Nieuwelink*, F. Meirer, L. Abelmann, M. Odijk, W. Olthuis, B.M. Weckhuysen, A. van den Berg, Magnetophoretic Sorting of Single Catalyst Particles, (2018) Angew. Chem. Int. Ed., 57 (33), pp. 10589-10594.

J.C. Vollenbroek*, A.-E. Nieuwelink*, J.G. Bomer, R.M. Tiggelaar, A. van den Berg, B.M Weckhuysen, M. Odijk, Droplet microreactor for reaction monitoring at elevated temperatures and pressure, (2018) 22nd International Conference on Miniaturized Systems for Chemistry and Life Sciences, MicroTAS 2018, 4, pp. 2131-2133.

*Authors contributed equally

\subsubsection{Co-author publications in peer reviewed journals}

M. Solsona, A.-E. Nieuwelink, M. Odijk, F. Meirer, L. Abelmann, W. Olthuis, B.M. Weckhuysen, A. van den Berg, Magnetophoretic sorting of fluid catalytic cracking particles, (2020) 21st International Conference on Miniaturized Systems for Chemistry and Life Sciences, MicroTAS 2017, pp. 1633-1634.

M. Solsona, A.-E. Nieuwelink, V.A. Papadimitriou, J.C.T. Eijkel, F. Meirer, L. Abelmann, W. Olthuis, B.M. Weckhuysen, A. van den Berg, Concentration polarization to measure nano-pore accessibility, (2020) 21st International Conference on Miniaturized Systems for Chemistry and Life Sciences, MicroTAS 2017, pp. 1497-1498.

R.G. Geitenbeek, B.B.V. Salzmann, A.-E. Nieuwelink, A. Meijerink, B.M. Weckhuysen, Chemically and thermally stable lanthanide-doped $\mathrm{Y}_{2} \mathrm{O}_{3}$ nanoparticles for remote temperature sensing in catalytic environments, (2019) Chem. Eng. Sci., 198, pp. 235-240.

M. Solsona, J.C. Vollenbroek, C.B.M. Tregouet, A.-E. Nieuwelink, W. Olthuis, A. van den Berg, B.M. Weckhuysen, M. Odijk, Microfluidics and catalyst particles, (2019) Lab Chip, 19 (21), pp. 3575-3601.

R.G. Geitenbeek, J.C. Vollenbroek, H.M.H. Weijgertze, C.B.M. Tregouet, A.-E. Nieuwelink, C.L. Kennedy, B.M. Weckhuysen, D. Lohse, A. van Blaaderen, A van den Berg, M. Odijk, A. Meijerink, Luminescence thermometry for: In situ temperature measurements in microfluidic devices, (2019) Lab Chip, 19 (7), pp. 1236-1246.

R.G. Geitenbeek*, A.-E. Nieuwelink*, T.S. Jacobs, B.B.V. Salzmann, J. Goetze, A. Meijerink, B.M. Weckhuysen, In Situ Luminescence Thermometry to Locally Measure Temperature Gradients during Catalytic Reactions, (2018) ACS Catal., 8 (3), pp. 2397-2401. 
E.S. Gutter $\phi$ d, S. Фien- $Ф$ degaard, K Bossers, A.-E. Nieuwelink, M. Manzoli, L. Braglia, A. Lazzarini, E. Borfecchia, S. Ahmadigoltapeh, B. Bouchevreau, B.T. Lønstad-Bleken, R. Henry, C. Lamberti, S. Bordiga, B.M. Weckhuysen, K.P. Lillerud, U. Olsbye, $\mathrm{CO}_{2}$ Hydrogenation over PtContaining Uio-67 Zr-MOFs - The Base Case, (2017) Ind. Eng. Chem. Res., 56 (45), pp. 1320613218.

J.C. Vollenbroek, J.G. Bomer, A. van den Berg, M. Odijk, A.-E. Nieuwelink, B.M. Weckhuysen, R.G. Geitenbeek, A. Meijerink, R.M. Tiggelaar, Design and characterization of a microreactor for monodisperse catalytic droplet generation at both elevated temperatures and pressures, (2017) 2017 IEEE 12th International Conference on Nano/Micro Engineered and Molecular Systems, NEMS 2017, art. no. 8017127, pp. 746-751.

F. Kirby, A.-E. Nieuwelink, B.W.M. Kuipers, A. Kaiser, P.C.A. Bruijnincx, B.M. Weckhuysen, CaO as Drop-In Colloidal Catalysts for the Synthesis of Higher Polyglycerols, (2015) Chem. Eur. J., 21 (13), pp. 5101-5109.

\subsubsection{Oral Presentations}

Microfluidic Sorting of Catalyst Particles based on Activity, EuropaCat, Aachen, Germany, 22-08-2019

Catalyst Particles and Microfluidics: Reaction, Separation and Analysis, NAM26, Chicago, IL, USA, 25-06-2019

A Multiphase Hydrogenation Reaction in a Droplet Microreactor, NCCC, Noordwijkerhout, The Netherlands, 06-03-2019

Magnetophoretic Sorting of Single Catalyst Particles, NCCC, Noordwijkerhout, The Netherlands, 07-03-2018

Single Particle Diagnostics: Integrating Catalysis and Optical Spectroscopy within a Microreactor, NCCC, Noordwijkerhout, The Netherlands, 06-03-2017

\subsubsection{Poster Presentations}

High-Throughput Droplet Microreactor for Catalytic Activity Screening of Solid Catalyst Particles, MicroTAS, Basel, Switzerland, 30-10-2019

Single Catalyst Particle Diagnostics: A Multiphase Hydrogenation Reaction in a Droplet Microreactor, NAM26, Chicago, IL, USA, 24-06-2019.

Single Particle Mapping: Correlating Multiple Diagnostic Tools for Catalyst Characterization, CHAINS, Veldhoven, The Netherlands, 06-12-2018, which has been awarded the best poster prize 


\subsubsection{Scientific contributions}

In this thesis, many chapters were established via collaborations. In this section, the shared contributions are listed per chapter.

\section{Chapter 2}

This chapter is based on the collaboration between the BIOS Lab-on-a-Chip group of the University of Twente, and the Inorganic Chemistry and Catalysis group from Utrecht University. Miguel Solsona (UT) developed the microreactor for magnetophoretic sorting. The collected samples were ex-situ analyzed in Utrecht using fluorescence microscopy, UV-vis spectroscopy, EPR, $\mu$-XRF and bright field microscopy.

\section{Chapter 3}

This chapter is based on the collaboration with Inorganic Chemistry and Catalysis colleague Marjolein Velthoen, who performed the bulk characterization of the density separated ECAT. Bachelor students Yoni Nederstigt and Kristel Jagtenberg were involved in the density separation and initial method development for single particle diagnostics respectively.

\section{Chapter 4 and 5}

These chapters are based on the collaboration between the BIOS Lab-on-a-Chip group of the University of Twente, and the Inorganic Chemistry and Catalysis group from Utrecht University. Both groups were responsible for the conceptual idea of the microreactors. Jeroen Vollenbroek (UT) developed and fabricated the microreactors with help from Johan Bomer (BIOS Lab-on-a-Chip group). Anne-Eva Nieuwelink helped in the development of the silicon/glass droplet microreactor and was involved in the calibration and measurements with the luminescent nanoparticles inside the droplet microreactor. She was also involved in the experiments in fluorescence activated catalyst sorting. Furthermore, the ex-situ post-sorting analysis using $\mu-X R F$, confocal fluorescence microscopy and optical micropscopy are carried out in Utrecht.

\section{Chapter 6}

This chapter is based on the collaboration between the BIOS Lab-on-a-Chip group of the University of Twente and the Inorganic Chemistry and Catalysis group from Utrecht University. Jeroen Vollenbroek co-supervised master student Andrea Ferreira de Abreu (ICC/UU) and was involved in the fabrication of the microreactor. All hydrogenation experiments and particle characterization were performed in Utrecht. Jeroen Vollenbroek was involved in the data analysis.

\section{Chapter 7}

This chapter is based on the collaboration between the BIOS Lab-on-a-Chip group of the University of Twente and the Inorganic Chemistry and Catalysis group from Utrecht University. Jeroen Vollenbroek co-supervised master student Wolfer van den Bosch (ICC/ $\mathrm{UU})$ who synthesized the fluorescent coumarin and performed the in-flow experiments. 


\subsection{Dankwoord}

Het is een bijzondere ervaring om jarenlang toe te werken naar één einddoel. Het was vreemd dat de afsluiting van die mooie jaren ineens een stukje opschoof, maar wat zijn nou een paar maanden uitstel op iets dat uiteindelijk een traject van bijna vijf jaar afsluit? Ik wil graag de tijd nemen om iedereen te bedanken die in welke vorm dan ook heeft bijgedragen aan dit proefschrift.

Ik heb het geluk gehad om met heel veel leuke, slimme, gezellige en inventieve mensen te mogen samenwerken. Jeroen, zonder jouw oneindig enthousiasme hadden we waarschijnlijk al jaren geleden moedwillig onze opstelling gesloopt en de zorgvuldig gesorteerde FCC deeltjes in het chemisch afval gedumpt. Ik zag altijd wel de meerwaarde van een dag hard werken zonder resultaat (je leert tenslotte erg veel over de opstelling waar je mee werkt), maar jij bent met stip op één degene die in alle pietluttige resultaten noemenswaardige vooruitgang zag en weigerde om te stoppen op een dieptepunt. 'We kunnen alles toch nog één keer schoonmaken? En dan proberen we nog één keer of de deeltjes dan wel in de chip willen'. Ondanks onze verschillende wetenschappelijke talen was het altijd erg fijn samenwerken. Het zal wel niet voor niets zijn dat we regelmatig als 'wetenschappelijk getrouwd' zijn bestempeld en ik ben dan ook blij met jou als paranimf. Dankjewel voor al het gepruts op de vierkante millimeter en je geduld om samen twee wetenschappelijke 'werelden' nader tot elkaar te brengen.

Dan wil ik graag mijn andere paranimf bedanken voor van alles eigenlijk. Voor het kamerdelen op conferenties, haarproducten ruilen (hij is nog steeds niet op), schelden op de tegenwind tijdens het naar huis fietsen, sociale interactie hekelen als je voeten het begeven op een netwerkborrel, kaasstengels kopen tijdens de lunch, rammelen met je theemok als het DDW nog leeg was 's ochtends, meezingen op het lab, je nuchtere kijk op het $\mathrm{PhD}$ leven en je blije persoonlijkheid. Oja, en ook nog voor de fruitful collaboration! Zijn we nou gaan samenwerken omdat we vriendinnen werden, of was het puur een wetenschappelijke interesse? Ik weet het niet, en het boeit niet. Het was gezellig in het donker achter de Renishaw, ook al vergeet ik de geur van 4-propylguaiacol nooit meer. Carolien, hartelijk dankjewel!

Bert, bedankt voor het vertrouwen in mij als persoon en de hulp bij het ontwikkelen tot zelfstandig onderzoeker. Het bleek een schot in de roos om mij te motiveren richting het MCEC project dat ik uiteindelijk zo ontzettend leuk heb gevonden om te doen. Het was soms lastig laveren tussen intensief samenwerken met 'Twente' en mijn eigen ding blijven doen, maar ik ben zelf in ieder geval tevreden met de uitkomst. Bedankt voor je kritische blik en je drive om overal het onderste uit de kan te willen halen. Ook de ruimte die ik kreeg om binnen MCEC mijn weg te vinden in het C-team, als student representative en het afgelopen jaar als projectleider, waardeer ik enorm.

Een samenwerkingsproject vraagt om begeleiding uit alle windstreken. Mathieu, dankjewel dat je me onder je hoede hebt genomen. Ik voelde me mede door jou erg thuis bij BIOS. Verder praat je met zoveel enthousiasme en energie over je vak, dat het haast onmogelijk is om dat niet over te nemen. Ik had me geen betere begeleider-op-afstand 
kunnen wensen. Over enthousiasme gesproken: Florian, I believe there's nothing too 'out of the box' for you to dismiss it without trying. Thank you for taking the time to repeatedly explain Matlab or to make changes in your script (I've become very fond of processXRFdataset.m) to make data-analysis-life more easy. Albert, dankjewel dat je Jeroen en mij zo onze gang hebt laten gaan. Voor mijn gevoel was je bij elke kleine update enthousiast, vooral over hetgeen we nog zouden kunnen bereiken in de nabije toekomst.

Miguel, thank you for the collaboration that lead to my first experimental chapter. It was a pleasure to make the first connections between BIOS and ICC by analyzing your carefully sorted particles. Together with Jeroen, we formed the 'single particle diagnostics platform' and apart from presenting the same results in a session of NCCC, I've always enjoyed our group-within-a-group feeling.

Marjolein, wat begon met het 'uitlenen' van wat FCC deeltjes van Yoni aan Kristel, eindigde in een intensieve samenwerking en een bijna-worsteling om die grote hoeveelheden data voor een paper te stroomlijnen. Het was heerlijk om op het lab als een stelletje eerstejaars samen te knutselen met het meest basic experiment. Dankjewel voor je goede inzichten wat betreft schrijven, en je fijne visie op de toekomst.

Ik vond het een voorrecht om naast mijn eigen onderzoek ook te mogen aanhaken bij iemand met ervaring in het PhD-schap. Robin, dankjewel dat je me meenam in de wondere wereld van de thermoluminescentie en de zeldzame aarden. Ik vond het ontzettend leuk om mee te mogen werken om mijn kennis in te zetten voor jouw project en ik vind het heel waardevol dat we elkaar daarna met zoveel kleine 'projectjes' nog telkens tegen gekomen zijn.

Andrea, as my first master student you were pioneering the field of microfluidics like a pro. Thank you for your great efforts that lead to Chapter 6. Wolfer, je systematische manier van werken, je pagina's vol voorbereide vragen bij een van onze overleggen en je verwondering over het doen van onderzoek maakten het erg leuk om je te begeleiden. Dankjewel voor al je inspanningen die hebben geleid tot Hoofdstuk 7. Peter, dankjewel voor je zelfstandigheid, je eigenwijze visie die leidde tot bijzondere resultaten, en je open gesprekken. Ik heb door jou erg veel geleerd over mijn manier van begeleiden. Kristel, Thimo en Tjom, het was erg leuk om jullie wegwijs te mogen maken in de labs van ICC en te zien hoe jullie omgingen met jullie eerste grote onderzoeksproject. Bedankt voor jullie bijdragen aan mijn onderzoek en de fijne samenwerking. Bas, je was het eerste bruggetje tussen Robin en mijzelf. Ik wil je bedanken voor je flexibiliteit en je zelfstandigheid wanneer ik weer eens aan je lot overliet vanwege mijn (altijd) overvolle agenda.

Daan, het voelde eigenlijk nooit onwennig om samen met jou het MCEC office te runnen. Het is fijn om met je samen te werken, dagelijkse beslommeringen te bespreken of te lachen tijdens kantooryoga.

Jongens, hartelijk dankjewel voor alle lunchrondjes, het bowlingadvies, de te lange theepauzes, het incidenteel samen eten, het kanoën, verjaardagsfeestjes en andere rare uitspattingen. Petra, Thomas, Ward, Katinka, Tom, Wouter, Joris, Robin, Daan, en Carolien: het werk was niet zo leuk geweest zonder jullie als collega's. 
Tijdens mijn PhD heb ik met veel verschillende mensen een 'kantoor' (bureaucluster in het open office) gedeeld. Ik ga vast mensen vergeten als ik alle ICC'ers op een rij wil zetten, maar overal was het even gezellig, en overal voelde ik me thuis. Bedankt voor de leuke groepsuitjes, borrels, spontane discussies over niks en meezingsessies op het lab. Silvia en Thomas, jullie waren/zijn fijne buur-collega's. Thomas, een extra dankjewel voor je mooie figuur (pagina 145, Figuur 9.8 voor degenen die terug willen bladeren), Chem EurJ cover, en je fijne manier van werken daarbij.

Dan is er nog een team bij ICC dat altijd voor je klaar staat. Dankjulliewel Dymph, Ilonka, Ad E, Ad M, Oscar, Marjan, Ramon, Herrick, Pascal, Fouad en Hannie, voor al jullie hulp zowel voor als achter de schermen.

Je zou het soms bijna vergeten, maar werk is niet altijd het állerbelangrijkste in je leven. Ik ben dankbaar voor alle leuke, lieve vrienden die ik in de loop van de tijd bij elkaar gespaard heb. Het 27ste, mijn maatjes uut 't Noord'n, Tineke, de Helden, het balletfeestje dat Mizou heet, Zwingin, mijn oud-huisgenootjes van VLL62: dankjulliewel voor alle leuke uitjes, late avonden, sterke verhalen, lachbuien en gespreksonderwerpen die op repeat stonden. Ik wil Lies en Zwingin in het bijzonder bedanken voor alle fijne repetities die mijn hoofd zo heerlijk leeg konden maken. Eerst leeg... en dan weer vol... met pasjes, choreografieën en melodieën.

Utrecht is na elf jaar wel echt mijn thuis geworden, maar Hijken-thuis was en is een geweldig toevluchtsoord. Wanneer het te druk is om bij jullie langs te komen, mis ik het en wanneer we naar Hijken toe komen voelt het meestal als te kort. Dankjewel voor jullie oneindige support, ook waar het soms niet nodig is. Mama, een speciaal dankjewel voor de mooie thesisvoorkant.

Marijne, dankjewel voor je steun, je verwondering, je ondeugende snuitje en je rare ideeën. Niet iedereen is goed in 'zusje' zijn, maar jij bent er de beste in. Jut en jul gaan hopelijk nog vaak samen op pad. Lieve zus, ik heb oneindig veel bewondering voor je doorzettingsvermogen om van elk dalletje weer een piek te maken. Je bent een onderzoeker, entertainer, jonkie en raadgever. Je weet dat ik glim van trots als ik mijn (niet meer zo kleine) zusje weer ergens op een podium zie staan. We zullen hopelijk nog vaak oogcontact maken en dan ons best doen om niet dom te grijnzen.

Laurens, er is veel om je voor te bedanken maar laat ik beginnen bij de kritische noten die je plaatste tijdens het tegenlezen van mijn thesis. "Dat is mijn werk" zeg je dan. Ja dat weet ik, maar toch.. Fijn dat je al die pagina’s door wilde ploegen. "Altijd” zeg je dan. Het siert je hoeveel je voor anderen wil doen, uitzoeken of oplossen. We gingen van een eiland waar de buitenwereld niet leek te bestaan naar een paleisje vanwaar we samen met een nieuwe blik de wereld in kijken. Wie had gedacht dat we zomaar ineens maandenlang de hele dag samen zouden doorbrengen in ons thuiskantoor? Sorry voor het gefrustreerde gebabbel als iets me nerveus maakte of anders liep dan verwacht. Ach, je hoeft meestal toch niets te zeggen om mijn gedachten te ontwarren. Wat de toekomst ook zal brengen, laten we er samen met open vizier op afgaan.

Anne-Eva 


\subsection{Curriculum Vitae}

Anne-Eva Nieuwelink was born in Assen, The Netherlands, on the $20^{\text {th }}$ of July 1991 . After obtaining a high school diploma (VWO) in 2009, she graduated with a Bachelor of Science degree in chemistry from Utrecht University in 2012. Before starting her Master's studies in Nanomaterials: Chemistry and Physics, she first was a board member of the study association for chemistry students "PROTON". In 2013 she started with a Master thesis on the oligomerization of glycerol using $\mathrm{CaO}$ colloidal particles in the group of prof. Bert Weckhuysen. An internship at the University of Oslo, in the group of prof. Unni Olsbye followed. Anne-Eva

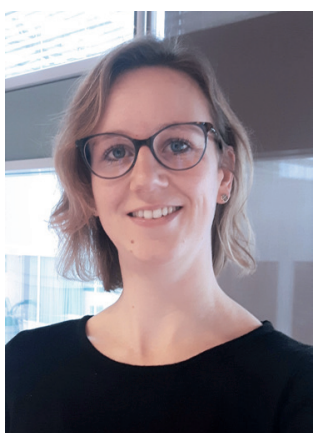
worked for 5 months on the catalytic properties of the MOF UiO-67 with incorporated Pt. In 2015 she graduated with a Master of Science degree in Nanomaterials Science, after which she started with her $\mathrm{PhD}$ research in the group of Inorganic Chemistry and Catalysis under supervision of prof. Bert Weckhuysen. An extensive collaboration with the BIOS Lab on a Chip group of prof. Albert van den Berg at the University of Twente lead to the work described in this $\mathrm{PhD}$ thesis and related published articles in scientific journals. In her free time, Anne-Eva enjoys dancing with the group 'Mizou Danst', singing with close harmony choir 'Zwingin' and photography. 
